SERVIÇO DE PÓS-GRADUAÇÃO DO ICMC-USP

Data de Depósito: 19/02/2004

Assinatura:

\title{
Equações de reação-difusão em domínios finos ${ }^{1}$
}

Olivâine Santana de Queiroz

Orientadora: Profa. Dra. Maria do Carmo Carbinatto

Dissertação apresentada ao Instituto de Ciências Matemáticas e de Computação da Universidade de São Paulo, como parte dos requisitos para obtenção do título de Mestre em Ciências - Area: Matemática.

USP - São Carlos
Fevereiro/2004

${ }^{1}$ Este trabalho teve suporte financeiro da FAPESP processo: 01/09954-0 
A Comissão Julgadora:

Profa. Dra. Laria do Carmo Carbinatto

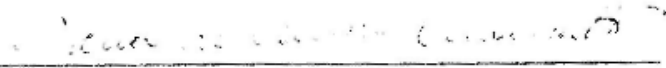

Prof. Dr. Alexandre Nolasco de Can'alho

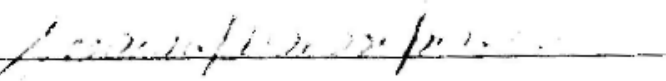

Prof. Dr. Gerson Petronilho

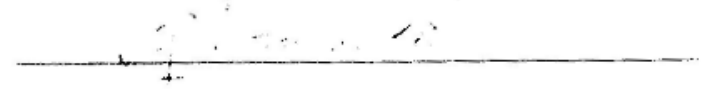


Aos meus pais, Oliveira e Vilma, com todo carinho, admiração e agradecimento eterno! 
"Nada acontece antes de ser sonhado"

(Carl Sandburg) 


\section{Agradecimentos}

As pessoas levam consigo as marcas do convívio diário com outras tantas pessoas. Sendo assim, agradeço a todos os meus amigos que me presenteam com momentos agradáveis. Além disso, muito agradeço:

à minha orientadora, Maria do Carmo, pela orientação, paciência, atenção e amizade, além da oportunidade que me deu de desenvolver esse trabalho:

aos meus pais, Oliveira e Vilma, cujo saber está no coração, pela confiança, constante incentivo e respeito com que têm acompanhado cada uma de minhas escolhas e aos meus irmãos, Gleizelane e Odevaine, pela amizade;

também aos meus sobrinhos, Gleiceane e Dyonatha, por serem motivos de ternura e alegria;

ao Neri e à sua família, pelo apoio incondicional e pela grande amizade que me têm dedicado;

a todos os meus amigos advindos com a graduação, em especial ao Cassio e ao João Paulo que continuam compartilhando comigo situações hilárias e outras tantas;

aos amigos de mestrado com os quais cursei disciplinas compartilhando dúvidas e conhecimentos e que, com sua amizade, tornaram este período muito prazeroso;

a todos os amigos da pós-graduação aqui do ICMC-USP, mestrandos e doutorandos, todos eles "titulares" em alegria e solidariedade;

a todos funcionários e professores aqui do ICMC-USP, em especial os professores com os quais cursei disciplinas e avancei um pouquinho no conhecimento da Matemática;

a todos aqueles que conviveram comigo nos tempos de Moradia Estudantil em Presidente Prudente, em especial os amigos Fernando e Marcel;

aos professores do Departamento de Matemática da FCT-UNESP de Presidente Prudente, onde essa "aventura" começou.

Sou grato ao ensino público e gratuito, o qual poucas pessoas têm acesso, mas que possibilitou todos os meus estudos até aqui.

Agradeço a DEUS, pelo dom da vida e por estar constantemente me provando que é possível crescer diante das dificuldades e também pela felicidade de poder compartilhar com todos a conquista de mais essa etapa importante em minha vida!

Olivâine 


\section{Resumo}

Seja $\Omega$ um domínio não-vazio limitado com fronteira Lipschitz arbitrário em $\mathbb{R}^{M} \times \mathbb{R}^{i r}$. Denotemos um ponto genérico de $\mathbb{R}^{M} \times \mathbb{R}^{N}$ por $(x, y)$. Dado $\varepsilon>0$, comprimimos o domínio $\Omega$ na direção $y$ obtendo o domínio $\Omega_{\varepsilon}:=\left\{(x, \varepsilon y) \in \mathbb{R}^{M} \times \mathbb{R}^{N} \mid(x, y) \in \Omega\right\}$. Nesse trabalho consideramos a família de equações de reação-difusão

$$
\begin{array}{ll}
u_{t}=\Delta u+f(u), & t>0,(x, y) \in \Omega_{\varepsilon}, \\
\partial_{\nu_{\varepsilon}} u=0, & t>0,(x, y) \in \partial \Omega_{\varepsilon},
\end{array}
$$

onde $f$ é uma não-linearidade com certas condições de crescimento que garantem que $\left(\tilde{E}_{\varepsilon}\right)$ gera um semifluxo $\tilde{\pi}_{\varepsilon}$ em $H^{1}\left(\Omega_{\varepsilon}\right)$. Essa família possui uma equação limite $\left(E_{0}\right)$, a qual gera um semifluxo limite $\ddot{\pi}_{0}$ definido em um subespaço fechado de $H^{1}(\Omega)$. Impondo uma condição de dissipatividade em $f$, para todo $\varepsilon \geq 0$, existe um atrator global $\mathcal{A}_{\tilde{\pi}_{\mathrm{s}}}$ associado ao semifluxo $\tilde{\pi}_{\varepsilon}$. Apresentamos importantes resultados sobre o comportamento assintótico da família de semifluxos $\left(\tilde{\pi}_{\varepsilon}\right)_{\varepsilon>0}$ quando $\varepsilon \rightarrow 0^{+}$. Em particular, mostramos que a família de atratores $\left(\mathcal{A}_{\dot{\pi}_{\varepsilon}}\right)_{\varepsilon \geq 0}$ é semicontínua superiormente em $\varepsilon=0$ num sentido apresentado no Capítulo 5. Finalizamos esse trabalho com um estudo da equação limite $\left(E_{0}\right)$ quando $M=N=1$ e $\Omega$ é um domínio bem decomposto. A elaboração dessa dissertação foi baseada nos artigos [20], [18] e [4]. 


\section{Abstract}

Let $\Omega$ be an arbitrary bounded nonempty domain in $\mathbb{R}^{M} \times \mathbb{R}^{N}$ with Lipschitz boundary. We denote by $(x, y)$ a generic point in $\mathbb{R}^{M} \times \mathbb{R}^{N}$. Given $\varepsilon>0$, we consider the squeezed domain $\widehat{\iota}_{\leq}:=\left\{i(x, \epsilon y) \in \mathbb{R}^{M} \times \mathbb{R}^{N} \mid(x, y) \in \Omega\right\}$. In this work we consider a family of reaction-diffusion equations

$$
\begin{array}{ll}
u_{t}=\Delta u+f(u), & t>0,(x, y) \in \Omega_{\varepsilon}, \\
\partial_{\nu_{\varepsilon}} u=0, & t>0,(x, y) \in \partial \Omega_{\varepsilon},
\end{array}
$$

where $f$ is a nonlinearity with some growth conditions such that for each $\varepsilon>0,\left(\tilde{E}_{\varepsilon}\right)$ generates a semiflow $\tilde{\pi}_{\varepsilon}$ in $H^{1}\left(\Omega_{\varepsilon}\right)$. This family has a limit equation $\left(E_{0}\right)$ which also generates a limiting semiflow $\pi_{0}$ defined on a closed subspace of $H^{1}(\Omega)$. A dissipativeness condition on $f$ implies that for each $\varepsilon \geq 0$, there exists a global attractor $\hat{\mathcal{A}}_{\tilde{w}}$ related to the semiflow $\tilde{\pi}_{\varepsilon}$. We present important results on the asymptotic behavior of the family of semiflows $\left(\tilde{\pi}_{\varepsilon}\right)_{\varepsilon>0}$ as $\varepsilon \rightarrow 0^{+}$. In particular, we show that the family of attractors $\left(\mathcal{A}_{\tilde{\pi}_{\varepsilon}}\right)_{\varepsilon \geq 0}$ is upper semicontinuous at $\varepsilon=0$ in a restrict sense to be presented in Chapter 5 . We conclude with a study of the limiting equation $\left(E_{0}\right)$ when $M=N=1$ and $\Omega$ is a nice decomposed domain. This work is based on the papers [20], [18] and [4]. 


\section{Conteúdo}

Introdução

1 Preliminares $\quad 5$

1.1 Teoria espectral abstrata . . . . . . . . . . . . . . . . 5

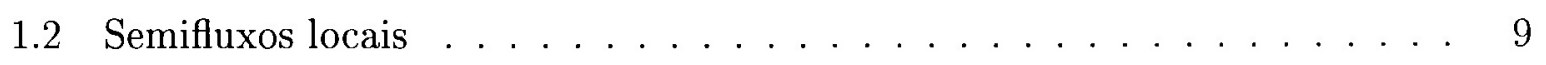

1.3 Atratores globais . . . . . . . . . . . . . . . . . 13

2 As Equações de Reação-Difusão em Domínios Finos

2.1 As equações de reação-difusão em domínios finos . . . . . . . . . . . . . . 19

2.2 Os espaços $H_{s}^{1}(O)$ e $L_{s}^{2}(O) \ldots \ldots \ldots \ldots \ldots \ldots \ldots$

2.3 o problema limite . . . . . . . . . . . . . . 36

3 Convergência Espectral $\quad 41$

3.1 Convergência espectral . . . . . . . . . . . . . . . . . 41

3.2 Monotonicidade . . . . . . . . . . . . . . . . . . 47

4 Convergência de Semigrupos e de Semifluxos 53

4.1 Convergência de semigrupos . . . . . . . . . . . . . . . . . . . . . . . . . 53

4.2 Convergência de semifluxos . . . . . . . . . . . . . . . . . . 59

5 Semicontinuidade Superior dos Atratores $\quad 69$

5.1 Existência de atratores . . . . . . . . . . . . . . . . . 69 69

5.2 Semicontinuidade superior dos atratores $\ldots \ldots \ldots \ldots 75$

6 Domínios bem Decompostos $\quad 77$

6.1 Domínios com seções verticais conexas . . . . . . . . . . . . . 77

6.2 Domínios bem decompostos . . . . . . . . . . . . . 85

6.3 Domínios em forma de $C \ldots \ldots \ldots \ldots . \ldots \ldots$

$\begin{array}{lr}\text { Bibliografia } & 105\end{array}$ 


\section{Introdução}

Problemas envolvendo domínios finos aparecem com certa naturalidade em situações físicas. Ao se cstudar a dinâmica de um oceano, por exemplo: a região onde se localiza o fluido possui um comprimento vertical fino quando comparado com as dimensões horizontais. Situação análoga aparece em Metereologia, onde podemos analisar problemas de fluidos circulando ontre cluas esforas concêntricas de raios $R$ e $R+\varepsilon$, as quais modelam a atmosfera. Neste caso, a diferença entre os raios pode ser considerada pequena ao ser comparada com a área da superfície terrestre. Há problemas envolvendo equações do reação-difusão para os quais conseguimos modelos com coeficientes de difusão grandes e, após uma mudança de variável, obtemos uma equação diferencial parcial definida em um domínio fino. Estes problemas dão origem a uma equação de evolução.

Em outras palavras, seja $Q \subset \mathbb{R}^{M}$ um domínio limitado com certa regularidade. Vamos supor que seja dada uma equação de evolução, com condições de fronteira do tipo Neumann, definida $\mathrm{em} Q \times(0, \Sigma)$ com $\Xi>0$. Em tal situação é natural pensarmos em ignorar a direção fina e considerar a equação apenas em $Q$. Espera-se, entretanto, que as informações qualitativas e quantitativas não sejam perdidas. Problemas envolvendo domínios finos têm sido considerados por diversos autores com a utilização de diferentes técnicas. Em [21] encontramos algumas referências de problemas que se enquadram nessa classe.

O estudo de equações diferenciais parciais definidas em domínios finos é um caso particular de problemas que envolvem os efeitos da variação do domínio na dinâmica de uma equação diferencial parcial. Na verdade, nessa classe de problemas a variação do domínio nào é "regular" e sua análise pode se tornar complicada. Nessa dissertação estudaremos um caso particular destes problemas.

Consideremos um domínio $\Omega$ não-vazio, limitado e com fronteira Lipschitz arbitrário em $\mathbb{R}^{M} \times \mathbb{R}^{N}$. Denotemos por $(x, y)$ um ponto qualquer de $\mathbb{R}^{M+N^{N}}$. Para cada $\Xi>0$ definimos $\left.\Omega_{\varepsilon}:=\{i x, \varepsilon y) \in \mathbb{R}^{M} \times \mathbb{R}^{N} \mid(x, y) \in \Omega\right\}$. $\Omega_{\varepsilon}$ é obtido comprimindo o domínio

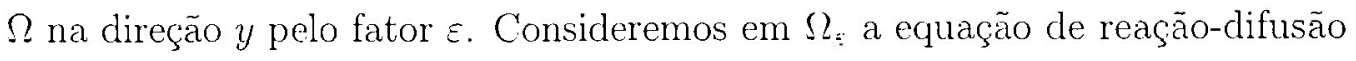

$$
\begin{array}{ll}
u_{t}=\Delta u+\int(u), & t>0,(x, y) \in \Omega_{\varepsilon} \\
\partial_{\nu_{\varepsilon}} u=0, & t>0,(x, y) \in \partial \Omega_{\varepsilon},
\end{array}
$$

onde $\nu_{\varepsilon}$ é a normal exterior a $\partial \Omega_{\varepsilon}$ e $f$ è uma não-linearidade. Aqui o domínio $\tilde{\Omega}=$ varia de acordo com o parâmetro $\varepsilon$. Nosso interesse é analisar o que ocorre com a dinâmica de 
$(\tilde{E})_{\Xi}$ quando $\varepsilon \rightarrow 0^{+}$. É conveniente realizarmos uma mundança de variável com a qual o parâmetro $\varepsilon$ é "transferido" para a equação do problema. De fato, há uma mudança de variável que transforma o domínio $\Omega_{\Sigma}$ no domínio fixado inicialmente $\Omega$, e a equação $\left(\bar{E}_{\varepsilon}\right)$ em uma equação $\left(E_{\varepsilon}\right)$. Mais precisamente:

$$
\begin{array}{ll}
u_{t}=\Delta_{x} u+\frac{1}{\iota^{2}} \Delta_{y} u+f(u), & t>0,(x, y) \in \Omega \\
\partial_{\nu_{x}} u+\frac{1}{\varepsilon^{2}} \partial_{\nu_{y}} u=0, & \iota>0,(x, y) \in \partial \partial \Omega,
\end{array}
$$

onde $\nu=\left(\nu_{x}, \nu_{y}\right)$ é a normal exterior em $\partial \Omega$. A pergunta repete-se: o que ocorre com a dinâmica de $(E)_{\varepsilon}$ quando $\varepsilon \rightarrow 0^{+*}$ ? Nosso próximo passo é definir una equação limite $\left(E_{0}\right)$ e comparar, de algum modo, a dinâmica da família $\left(E_{\varepsilon}\right), \varepsilon>0$, com a de $\left(E_{0}\right)$.

Se associado a cada $\left(E_{\varepsilon}\right), \varepsilon>0$, temos definido um atrator global $\mathcal{A}_{\pi_{\varepsilon}}$, podemos comparar a dinânica da família $\left(F_{\varepsilon}\right)_{\varepsilon>0} \mathrm{com}$ a dinâmica de $\left(E_{0}\right)$ analisando a semicontinuidade superior da família de atratores $\left(\mathcal{A}_{\pi_{\epsilon}}\right)_{2>0}$ em $\varepsilon=0$. Respostas para a pergunta acima prara equaçoes de reação-difusão en domínios finos foran estudadas em [14] o [20]. Os resultados apresentados em [20] possuem um caráter mais geral do que os encontrados em [14]. No caso biclimensional, um exemplo dos domínios consirlerados em [14] são os definidos por uma função suave $g$ definida em um intervalo fechado $[a, b]$, isto é,

$$
\Omega:=\left\{(x, y) \in \mathbb{R}^{2} \mid a<x<b, 0<y<g(x)\right\} .
$$

Hale e Raugel mostram em [11] que, nesse caso, a família de semifluxos $\left(\pi_{\varepsilon}\right)_{\varepsilon>0}$, onde para cada $\varepsilon>0, \pi_{\varepsilon}$ ć o semifluxo gerarto pelas soluçôes de $\left(F_{\varepsilon}\right)_{\varepsilon>0}$, possui um semiffuxo limite $\pi_{0}$, o qual é gerado pelo seguinte problema unidimensional em $[a, b]$ :

$$
\begin{aligned}
u_{t} & =(1 / g)\left(g u^{\prime}\right)^{\prime}+f(u), \\
u^{\prime}(c) & =0, \quad c=a, b .
\end{aligned}
$$

Impondo uma condição de dissipatividade em $f$, os autores também estabeleceram um resultado de semicontinuidade superior para a correspondente família de atratores.

Infelizmente, as técnicas utilizadas em [14] não podem ser generalizadas para o caso de domínios mais gerais, por exemplo, os que contêm "buracos". Prizzi e Rybakowski mostraram em [20] que, mesmo em situações mais complicadas, um semifluxo limite existe. Impondo certas condições de crescimento na função $f$, para cada $\varepsilon>0$, a equação $\left(E_{\varepsilon}\right)$ pode ser escrita na forma abstrata

$$
\dot{u}+A_{\bar{\varepsilon}} u=\hat{f}(u),
$$

onde $\dot{f}$ ć o operador de Nemitskir associado a $f$. Neste trabalho foram definidos um subespaço fechado de $H^{1}(\Omega)$, denotado por $H_{s}^{1}(\Omega)$, e também um subespaço fechado de $L^{2}(\Omega)$, denotado por $L_{u}^{2}(\Omega)$, o qual é o fecho de $H_{s}^{1}(\Omega)$ na norma de $L^{2}(\Omega)$. 
Com a definição de um operador linear $A_{0}: D\left(A_{0}\right) \subset H_{s}^{1}(\Omega) \longrightarrow L_{u}^{2}(\Omega)$, foi obtida uma equação abstrata:

$$
\dot{u}+A_{0} u=\hat{f}(u),
$$

onde, novamente, $\hat{f}$ é o operador de Nemitskii associado a $f$, o qual aplica subconjuntos de $I I_{s}^{1}(\Omega)$ em subconjuntos de $L_{s}^{2}(\Omega)$. Tal equação define um semifluxo $\pi_{0}$ no espaço $H_{s}^{1}(\Omega)$, que é o semifluxo limite para a família $\left(\pi_{\varepsilon}\right)_{z>0}$. Para comparar os semifluxos $\pi_{\varepsilon} \operatorname{com} \pi_{0}$, foi introduzida uma família de normas $|\cdot|_{\varepsilon}$ e um resultado de convergência com relação a essas normas é mostrado.

Impondo a condição de dissipatividade na não-linearidade $f$, temos que cada semifluxo $\pi_{\varepsilon}$, $\varepsilon \geq 0$, possui um atrator global $\mathcal{A}_{\overline{w_{i}}}$. Prizzi c Rybakowski mostraram em [20] que a família de atratores $\left(\mathcal{A}_{\tau}\right)_{->n}$ é semicontínua superiormente em $\varepsilon=0$ com relação a família de normas $|\cdot|_{\varepsilon}$. Estes resultados generalizam os resultados de [14]. Além disso, quando $M=N=1$ e sobre certas condições na geometria de $\Omega$, eles caracterizaram o operador $A_{0}$ e seu domínio $D\left(A_{0}\right)$.

Nosso estudo teve como ponto inicial o trabalho [20]. Também analisamos alguns resultados de [4] que versam sobre o comportamento da família de autovalores associados à família de problemas $\left(E_{z}\right)_{\varepsilon: 1)}$. Em [4], estes resultados foram utilizados no cálculo do Indice de Conlev de certos conjuntos invariantes isolados. Para nos auxiliar na análise do problema limite também estudamos alguns resultados de [18]. A caracterização do problema limite apresentada em [18] foi utilizada para demonstrar o Teorema da Variedade Incrcial. Tanto a Teoria do Índice de Conley quanto o Teorema da Variedade Inercial não fizeram parte do nosso projeto de estudo.

Passamos a descrever o modo em que esse texto está organizado.

No Capítulo 1 apresentamos algumas definições básicas necessárias no decorrer do texto. Introduzimos os conceitos já conhecidos de autovalor e autovetor de pares de formas bilineares simétricas, semifluxos em espaços métricos e atratores globais. Estes últimos são apresentados no contexto de equações diferenciais parabólicas.

O Capítulo 2 é dedicado à apresentação do problema de reação-difusão definido em domínios finos. Formulamos a equação abstrata associada ao problema $\left(\dot{E}_{\varepsilon}\right)$ bem como a equação correspondente $\left(E_{\Xi}\right)$, obtida por mudança de variável conveniente. Demonstramos resultados que nos garantem que tais equações geram semifluxos em $H^{1}(\Omega)$. Mostramos que a mudança de variáveis é, na verdade, uma conjugação entre os semifluxos gerados por $\left(\tilde{E}_{\varepsilon}\right)$ e $\left(E_{\bar{\varepsilon}}\right)$. Os espaços "limites" $H_{s}^{1}(\Omega)$ e $L_{s}^{2}(\Omega)$ são apresentados e estudamos algumas propriedades dos mesmos. Por último, constrúmos o problema limite. Para tanto, são necessários alguns resultados adicionais sobre a função $f$ que garantom que o scmifluxo "limite" esteja bem definido no espaço $H_{s}^{1}(\Omega 2)$. Para comparar tais semifluxos, introduzimos uma família de normas $|\cdot|_{\varepsilon:} \varepsilon>0$.

O resultado de convergência dos autovalores e autovetores é apresentado no Capítulo 3. Neste capítulo também apresentamos un resultado de [4] que trata sobre uma mono- 
tonicidade estrita da família de autovalores.

Tendo provado a convergência dos autovalores e autovetores, estamos prontos para "atacar" a convergência rlos semigrupos lineares e dos semifluxos. Isso c fcito no Capítulo 4. Demonstramos que existe uma certa convergência uniforme dos semigrupos lineares com relação à família de normas $|\cdot|_{\varepsilon}, \varepsilon>0$. A seguir mostramos a convergência dos semifluxos. Finalizamos com um resultado sobre as soluções associadas aos semifluxos que muito nos auxiliará na análise da semicontinuidade superior dos atratores.

O Capítulo 5 trata da semicontinuidade superior $\mathrm{em} \varepsilon=0$ da família de atratores $\left(\mathcal{A}_{\pi_{\varepsilon}}\right)_{\varepsilon>0}$. Para que esse estudo seja feito é necessário antes garantir a existência dessa família, o que é feito acrescentando uma condição de dissipatividade na não-linearidade $f$.

Em [18] os autores fazem uma análise mais cautelosa dos espaços $H_{s}^{1}(\Omega)$ e $L_{s}^{2}(\Omega)$ no caso em que $\Omega$ é um domínio com seções verticais conexas e, posteriormente, no caso mais geral em que $\Omega$ é um domínio bem decomposto, mas sempre com $M=N=1$. Esta anćlise, bem como a definição de domínios bem decompostos, é apresentada no Capítulo 6. O importante teorema de caracterização do problema limite também é apresentado neste capítulo. Finalizamos o capítulo ilustrando o teorema que caracteriza o operador linear limite com os domínios em forma de $C$. Para estes, apresentamos um estudo dos autovalores do operador linear limite. 


\section{Capítulo 1}

\section{Preliminares}

Nesse primeiro capítulo iremos apresentar algumas definições e resultados que serão utilizados no decorrer desse trabalho. A Seção 1.1 traz um estudo sobre os autovalores e autovetores associados a um par $(a, b)$ de formas bilineares. Nesta mesma seção também apresentamos importantes resultados sobre os operadores gerados por estas formas bilineares. Na Seção 1.2 a definição c alguns resultados sobre semifluxos são apresentados. Os atratores globais, juntamente com alguns resultados, são apresentados na Seção 1.3 no contexto das equações parabólicas semi-lineares. Para os interessados em uma bibliografia, indicamos [8], [23] e [28] para o estudo da teoria espectral. Os semifluxos em espaços métricos tal como são apresentados aqui se encontram em [25]. No estudo dos atratores globais citamos [13], [16] e [22]. Nesse capítulo e em todo texto utilizamos definições, notações e resultados sobre equações diferenciais parciais de [15].

\section{$1.1 \quad$ Teoria espectral abstrata}

Nessa seção apresentaremos a definição de autovalores e autovetores para pares de formas bilineares simétricas e alguns resultados relacionados com este conceito. As demonstrações podem ser encontradas em [8], [23] ou [28].

Definição 1.1.1 Seja $V$ um espaço vetorial sobre $\mathbb{R}$ e considere $a: V \times V \longrightarrow \mathbb{R}$ e $b: V \times V \longrightarrow \mathbb{R}$ formas bilineares simétricas em $V$. Se $\lambda \in \mathbb{R}$ e $u \in V$, com $u \neq 0$, satisfazem

$$
a(u, v)=\lambda b(u, v), \text { para todo } v \in V,
$$

dizemos que $\lambda$ é um autovalor do par $(a, b)$ e que $u$ é um aulovetor do par $(a, b)$ correspondente ao autovalor $\lambda$. Também dizcmos que $(\lambda, u)$ é um par autovalor-autovetor de $(a, b)$.

Sejam $V$ e $H$ dois espaços de Hilbert sobre $\mathbb{R}$ de dimensão infinita. Vamos supor também que $V \subset H$ com inclusão compacta e que $V$ seja denso em $H$. Denotaremos por 
$|\cdot|_{V}$ e $|\cdot|_{H}$ as normas de $V$ e $H$ respectivamente, $\langle\cdot, \cdot\rangle$ o produto interno de $H$. Seja $b$ a restrição deste produto interno a $V \times V$. É claro que o produto interno $b$ pode ser visto como uma forma bilinear simétrica em $V \times V$.

Uma forma bilinear simétrica e contínua $a: V \times V \longrightarrow \mathbb{R}$ é chamada coerciva com relação a $H$, ou somente coerciva, se satisfaz a scguinte condição: existem constantes $d$, $\alpha \in \mathbb{R} \operatorname{com} \alpha>0$ tais que

$$
a(u, u) \geq \alpha|u|_{V}^{2}-d|u|_{H}^{2} \text { para todo } u \in V .
$$

O result ado a seguir, cuja demonstração pode ser encontrada em [23] e [8], apresenta algumas propriedades dos antovalores de um par $(a, b)$, onde $b$ é como acima e $a: V \times V \longrightarrow$ $\mathbb{R}$ é uma forma bilinear contínua, simétrica e coerciva. Mais precisamente:

Proposição 1.1.2 Sejam $V$ e H espaços de Hilbert como acima eb a restrição do produto interno de $H$ a $V \times V$. Seja $a: V \times V \rightarrow \mathbb{R}$ uma forma bilinear simétrica, contínua $e$ coerciva e consideremos o par $(a, b)$. Então, o conjunto dos autovalores de $(a, b)$ é infinito e enumerável, possui um menor elemento e cada autovalor possui multiplicidade finita. Além disso, se $\left(\lambda_{k}\right)_{k \in \mathbb{N}}$ é esta sequência de autovalores, entäo $\lambda_{k} \rightarrow \infty$ quando $n \rightarrow \infty$.

Dizemos que uma sequência $\left(u_{n}\right)_{n \in \mathbb{N}} \mathrm{em} V$ ć $I I$-ortogonal se $\left\langle u_{i}, u_{j}\right\rangle=0$ sempre que $i \neq j$. A sequência $H$-ortogonal $\left(u_{n}\right)_{n \in \mathbb{N}}$ é dita $I I$-completa se sempre que $\left\langle u_{i}, v\right\rangle=0$ para todo $i \in \mathbb{N}$, então $v=0$.

Para cada $k \in \mathbb{N}$, o conjunto de todos us subespaços vetoriais $k$-dimensionais de $V$ será denotado por $\mathcal{V}_{k}$.

No restante dessa seção, seja $a: V \times V \longrightarrow \mathbb{R}$ uma forma bilinear, simétrica, contínua e coerciva e sejam $V, H$ e $b$ como na Proposição 1.1.2. Denotemos por $\left(\lambda_{n}\right)_{n \in \mathbb{N}}$ a sequência repetida dos autovalores de $(a, b)$.

O próximo resultado caracteriza cada um dos autovalores do par $(a, b)$.

Proposição 1.1 .3 ([23], Teorema 6.2 2) Para cada $k \in \mathbb{N}$,

$$
\lambda_{k}=\min _{E \in \mathcal{V}_{k}} \max _{u \in E \backslash\{0\}} \frac{a(u, u)}{b(u, u)} .
$$

Além disso, existe uma sequência $\left(u_{n}\right)_{n \in \mathbb{N}} H$-ortogonal tal que para cada $k \in \mathbb{N}$, $u_{k}$ é um autovetor de $(a, b)$ correspondente ao autovalor $\lambda_{l}$.

Notemos que a Proposição 1.1.3 implica que a sequência de autovalores de $(a, b)$ satisfaz

$$
0<\lambda_{1} \leq \lambda_{2} \leq \lambda_{3} \leq \cdots
$$

Outro modo de caracterizar os autovalores é dado a seguir. 
Proposição 1.1.4 ([23]) Se $\left(u_{n}\right)_{n \in \mathbb{N}}$ é uma sequência $H$-ortogonal tal que para cada $k \in$ $\mathbb{N}, u_{k}$ é autovetor de $(a, b)$ associado ao autovalor $\lambda_{k}$, então $\left(u_{n}\right)_{n \in \mathbb{N}}$ é $H$-completa e para cada $k \in \mathbb{N}$ com $k \geq 2$,

$\lambda_{k}=a\left(u_{k}, u_{k}\right) /\left|u_{k}\right|_{H}^{2}$

$=\min \left\{a(u, u) /|u|_{H}^{2} \mid u \in V \backslash\{0\} \operatorname{com}\left\langle u, u_{j}\right\rangle=0 \operatorname{para} j=1, \ldots, k-1\right\}$.

Se $k=1$ então

$$
\lambda_{1}=\min \left\{a(v, v) /|v|_{H}^{2} \mid v \in V \backslash\{0\}\right\} .
$$

Além disso, o espaço $V$ é caracterizado por

$$
V=\left\{\left.v \in H\left|\sum_{n=1}^{\infty} \lambda_{n}\right|\left\langle v, u_{n}\right\rangle\right|^{2} /\left|u_{n}\right|_{H}^{2}<\infty\right\} .
$$

A seguinte recíproca da Proposição 1.1.4 é válida.

Proposição 1.1.5 Suponhamos que exista uma sequência de números reais $\left(\mu_{n}\right)_{n \in \mathbb{N}} e$ uma sequência de vetores $\left(v_{n}\right)_{n \in \mathbb{N}} I I$-ortogonal em $V \backslash\{0\}$ tais que, para cada $k \in \mathbb{N}$ com $k \geq 2$,

$$
\begin{aligned}
\mu_{k} & =a\left(v_{k}, v_{k}\right) /\left|v_{k}\right|^{2} \\
& =\min \left\{a(u, u) /|u|^{2} \mid u \in V \backslash\{0\} \operatorname{com}\left\langle u, v_{j}\right\rangle=0 \text { para } j=1, \ldots, k-1\right\},
\end{aligned}
$$

e para $k=1$.

$$
\mu_{1}=\min \left\{a\left((i, v) /|v|_{H}^{\bar{z}_{H}} \mid v \in V \backslash\{0\}\right\} .\right.
$$

Então, para cada $k \in \mathbb{N}$, temos que $\mu_{k}=\lambda_{k}$ e $v_{k}$ é um autovetor de $(a, b)$ correspondente ao autovalor $\mu_{k}$.

Demonstração. Segue do Teorema 2.3 em [28] que, para cada $k \in \mathbb{N}$, $v_{k}$ ć autovetor do par (a.b) associado ao autovalor $\mu_{k}$ e o Teorema 3.1 em [28] implica que a sequência $\left(v_{k}\right)_{k \in \mathbb{N}}$ é $H$-completa. Além disso,

$$
0<\mu_{1} \leq \mu_{2} \leq \mu_{3} \leq \cdots
$$

Assim, para completar a demonstração da proposição precisamos mostrar que $\mu_{k}=\lambda_{k}$ para todo $k \in \mathbb{N}$. Dado $v \in V$ com $v \neq 0$, utilizaremos a notação $R(v):=a(v, v) /|v|_{H}^{2}$. Assim, para todo $k \in \mathbb{N}, R\left(v_{k}\right)=\mu_{k}$. É claro que $\iota_{1}=\lambda_{1}$. Denotemos por $V_{m}$ o subespaço de $V$ gerado pelos vetores $v_{1}, \ldots, v_{m}$. Seja $v \in V_{m} \operatorname{com} v \neq 0$ arbitrário. Logo, existem números reais $\alpha_{1}, \ldots, \alpha_{m}$ tais que $v=\sum_{i=1}^{m} \alpha_{i} v_{i}$. Portanto, (1.1) implica que

$$
R(v)=\frac{\sum_{i=1}^{m} \mu_{i} \alpha_{i}^{2}}{\sum_{i=1}^{m} \alpha_{i}^{2}} \leq \mu_{m} .
$$


Como $R\left(v_{m}\right)=\mu_{m}$, temos que

$$
\iota_{m}=\max _{v \in V_{m} \backslash\{0\}} R(v)
$$

Seja agora $F_{m}$ um subespaço de $V$ de dimensão $m$ arbitrário. Afirmamos que

$$
\mu_{n} \leq \max _{v \in E_{i n} \backslash\{0\}} R(v) .
$$

Isso é claro se $E_{m}=V_{m}$. Suponhamos então que $E_{m} \neq V_{m}$. Então existe um $v_{0} \in E_{m}$ com $\left\langle v_{0}, v_{i}\right\rangle=0$ para $i=1, \ldots, m-1$. Com isso, temos que $\mu_{m} \leq R\left(v_{0}\right)$ e portanto $\mu_{m} \leq \max _{v \in E_{m} \backslash\{0\}} R(v)$, demonstrando nossa afirmação

Nossa afirmação implica que, para cada $k \in \mathbb{N}$

$$
\mu_{k}=\min _{E \in \mathcal{V}_{k}} \max _{u \in L \backslash\{0\}} \frac{a(u, u)}{b(u, u)}=\lambda_{k} .
$$

Isso prova a proposição

Seja $W$ o conjunto de todos $u \in V$ tais que existe $u m=w_{u} \in H$ com a propricdade

$$
a(u, v)=\langle w, v\rangle \text { para todo } v \in V .
$$

Os resultados da Seção 3.2.5 do Capítulo VI em [7] mostram que $W \neq \emptyset$ e que, dado $u \in W$, o vetor $w_{u}$ é determinado de modo único. Assim, podemos definir o seguinte operador linear

$$
A: D(A) \subset V \longrightarrow V,
$$

dado por $A u:=w_{u}, u \in D(A):=W$. Além disso, as Proposições 9 e 10 da Seção 3.2.5, Capítulo VI de [7] implicam no seguinte resultado:

Proposição 1.1.6 $O$ conjunto $D(A)$ é um subespaço vetorial denso em $V$ e em $H$. O operador $A$ é auto-adjunto em $(H,\langle\cdot, \cdot\rangle)$ com resolvente compacto.

O operador $A$ definido na Proposição 1.1 .6 é chamado operador linear gerado pelo $\operatorname{par}(a,\langle\cdot, \cdot\rangle)$. E claro que um par $(\lambda, u)$ c um par autovalor-autovetor de $A$ se, e somentc se, $(\lambda, u)$ é um par autovalor-autovetor de $(a, b)$.

Concluímos essa seção com uma importante propriedade do operador $A$ gerado por un par de formas bilineares satisfazendo as propriedades acima.

Os conceitos e resultados sobre semigrupos analíticos, operadores setoriais e os importantes espaços de potências fracionárias podem ser encontrados em [12], [15] ou [17]. Seguiremos aqui a notação de [15]. Para uma apresentação mais detalhada destes tópicos sugerimos [5] e [6]. O próximo resultado é uma consequência, por exemplo. da Proposição 3, da Scção A6, Capítulo XVII cm [9]. 
Proposição 1.1.7 O operador linear A gerado pelo par $(a, b)$ é um operador setorial em $H$ e, portanto, $-A$ é o gerador infinitesimal de um semigrupo analítico, o qual é denotado por $\left\{e^{-A t}, t \in[0, \infty)\right\}$.

Além disso, segue: por exemplo, de resultados da Seção 3.6 do Capítulo VIII de [8] o seguinte fato:

Proposição 1.1.8 Para cada $\alpha>0$, seja $H^{\alpha}$ o espaço de potência fracionária associado ao operador $A$. Se $\alpha=1 / 2$, então

$$
H^{1 / 2}=V
$$

Temos também a seguinte relação entre os autovalores de $A$ e do semigrupo $\left\{e^{-A t}, t \in\right.$ $[0, \infty)\}$ por ele gerado:

Proposição 1.1.9 ([17], Teorema 2.4) Com a notaçũo introduzida nessa seçüo, valem as seguintes propriedades:

(1) se $\lambda \dot{e}$ um autovalor de $A$, então, para cada $l>0$, e $e^{-\lambda t}$ é autovalor de $e^{-A t}$;

(2) seja $t>0$. Se $\mu=e^{-\lambda t}$ é um autovalor de $e^{-A t}$, então existe um $k \in \mathbb{Z}$ tal que $\lambda+2 \pi k i / t$ é um autovalor de $A$.

\subsection{Semifluxos locais}

Nessa seção apresentamos o conceito de semifluxo local. Utilizaremos a notação apresentada em [25].

Definição 1.2.1 Sejam $X$ um espaço métrico, $D$ um subconjunto aberto em $[0, \infty) \times X$ e $\pi: D \longrightarrow X$ una aplicação. Se $(l, x) \in D$, escreveremos $x \pi t:=\pi(t, x)$. A aplicação $\pi$ é chamada semifluxo local se as seguintes propriedades estão satisfeitas:

(1) $\pi$ é uma aplicação contínua;

(2) para todo $x \in X$, existe um $\omega_{x} \in(0, \infty]$ tal que $(t, x) \in D$ se, e somente se, $0 \leq t<\omega_{x}$

(3) $x \pi 0=x$, para todo $x \in X$;

(4) se $(t, x) \in D$ e $(s, x \pi t) \in D$, então $(t+s, x) \in D$ e $x \pi(t+s)=(x \pi t) \pi s$.

Se $\omega_{t}=\infty$ para todo $x \in X$, então $\pi$ é chamado um semifluxo global.

Se $A \subset X$ e $S \subset[0, \infty)$ são tais que $S \times A \subset D$, definimos $A \pi S:=\pi(S \times A)$. Além disso, se $A=\{x\}$ ou $S=\{t\}$, escrevemos $x \pi S:=\{x\} \pi S$ e $A \pi t:=A \pi\{t\}$. 
Exemplo 1.2.2 ([15], Teoremas 3.3.3 e 3.4.1) Suponhamos que $X$ seja um espaço de Banach e seja $A: D(A) \subset X \longrightarrow X$ um operador setorial. Seja $0 \leq \alpha<1$ e consideremos um conjunto aberto $U \subset X^{\alpha}$ e $f: U \longrightarrow X$ uma função localmente Lipschitziana. O Teorema 3.3.3 em [15] implica que, para cada $u_{0} \in U$, existe um $\omega_{u_{0}} \in(0, \infty]$ tal que o problema de valor inicial

$$
\left\{\begin{array}{l}
\dot{u}+A u=f(u), \\
u(0)=u_{0} .
\end{array}\right.
$$

possui uma única solução $t \mapsto u\left(t, u_{0}\right)$ definida em $\left[0, \omega_{u_{0}}\right)$. Escrevendo $u_{0} \pi_{f} t:=u\left(t, u_{0}\right)$, segue que $\pi_{f}$ é um semifluxo local em $U$.

Dado um semifluxo local $\pi$, temos associado a este semifluxo o conceito de soluçĩo.

Definição 1.2.3 Seja $\pi$ um semifluxo local e $J$ um intervalo em $\mathbb{R}$. Uma aplicação $\sigma: J \longrightarrow X$ é chamada uma solução de $\pi$ se, para todo $t \in J$ e $s \in[0, \infty)$ tais que $t+s \in J$, segue que $\sigma(t) \pi s$ está definido e $\sigma(t) \pi s=\sigma(t+s)$. Se $0 \in J$ e $\sigma(0)=x$, dizemos que $\sigma$ é uma solução passando por $x$. Se $J=\mathbb{R}$, então $\sigma$ é chamada uma solução completa com relação ao semifluxo local $\pi$ (passando por $x=\sigma(0)$ ).

Exemplo 1.2.4 Consideremos a notaşão introduzida no Exemplo 1.2.2. Seja $J \subset \mathbb{R}$ um intervalo aberto e $\sigma: J \longrightarrow U$ uma solução de $\pi_{f}$. Afirmamos que $\sigma$ é diferenciável $\mathrm{cm}$ $J$. De fato, seja $s_{0} \in J$ e fixemos $s_{1} \in J$ com $s_{1}<s_{0}$. Definamos $\tilde{r}:=\left(s_{0}-s_{1}\right) / 2$. Logo, se $|h|<\tilde{r}$, temos que $s_{0}+h>s_{1}$. Como $J$ é um intervalo aberto, tomando $r>0$ suficientemente pecueno, podenos assunir também que $s_{0}+h \in J$ para todo $h \in \mathbb{R}$ com $|h|<r$.

Dado $h \in \mathbb{R}$ com $|h|<r$, definimos $t:=\left(s_{0}-s_{1}\right)+h$ e $t_{1}:=s_{0}-s_{1}$. Segue que $l>0$ e $t_{1}>0$. Além disso, como $s_{1}+t=s_{0}+h \in J$ e $s_{1}+t_{1}=s_{0} \in J$, temos que

$$
\sigma\left(s_{1}\right) \pi_{f} t=\sigma\left(s_{1}+t\right)=\sigma\left(s_{0}+h\right),
$$

$$
\sigma\left(s_{1}\right) \pi_{f} t_{1}=\sigma\left(s_{1}+t_{1}\right)=\sigma\left(s_{0}\right) .
$$

Definindo $u_{0}:=\sigma\left(s_{1}\right) \in U$, temos que $u_{0} \pi_{f} l$ e $u_{0} \pi_{f} t_{1}$ estão definidas $\mathrm{e}$

$$
\frac{\sigma\left(s_{0}+h\right)-\sigma\left(s_{0}\right)}{h}=\frac{u_{0} \pi_{f} l-u_{0} \pi_{f} \iota_{1}}{t-t_{1}} .
$$

Se $h \rightarrow 0$ temos que $t \rightarrow t_{1}$. Como a função $t \mapsto u_{0} \pi_{f} t$ é diferenciável em $t_{1}$, segue que $s \mapsto \sigma(s)$ é diferenciável em $s_{0}$.

Outro conceito importante associado a semifluxos (locais ou globais) é o de conjuntos invariantes. 
Seja $Y$ um subconjunto de $X$ e consideremos os seguintes conjuntos:

$$
\begin{aligned}
\operatorname{Inv}_{\pi}^{+}(Y):= & \left\{x \in X \mid x \pi\left[0, \omega_{x}\right) \subset Y\right\}, \\
\operatorname{Inv}_{\pi}^{-}(Y):= & \{x \in X \mid \text { existe uma solução } \sigma:(-\infty, 0] \rightarrow X \\
& \text { passando por } x \operatorname{com} \sigma(-\infty, 0] \subset Y\}, \\
\operatorname{Inv}_{\pi}(Y):= & \operatorname{Inv}_{\pi}^{+}(Y) \cap \operatorname{Inv}_{\pi}^{-}(Y) .
\end{aligned}
$$

Um conjunto $Y \subset X$ é chamado invariante se $Y=\operatorname{Inv}_{\pi}\left(Y^{\prime}\right)$.

Notemos que se $Y$ ć um subconjunto do $X$ e $\pi$ um scmifluxo local $\mathrm{cm} X$ tais que $\omega_{x}=\infty$ para todo $x \in Y$, então $Y$ é invariante se, e somente se, para todo $x \in Y$, existe uma solução completa $\sigma: \mathbb{R} \longrightarrow X$ passando por $x$.

Seja agora $N$ um subconjunto arbitrário do espaço métrico $X$. Dizemos que o semifluxo $\pi$ não explode em $N$ se, para todo $x \in X$ tal que $x \pi\left[0, \omega_{x}\right) \subset N$, temos que $\omega_{x}=\infty$.

Lema 1.2.5 Sejam $X, A$ ef como no Exemplo 1.2.2 e $N \subset U$ um subconjunto fechado em $X^{\alpha}$ e limitado em $X$. Se $f(N)$ i limitado, então o semifluxo local $\pi_{f}$ não explode em $N$.

Demonstração. Suponhamos que a conclusão do teorema não seja verdadeira. Logo, existe um $u_{0} \in X \operatorname{com} u_{0} \pi_{f}\left[0, \omega_{u_{0}}\right) \subset N$ e $\omega_{u_{0}} \in(0, \infty)$. Afimamos que existe um $u_{1} \in N$ tal que $\left|u_{0} \pi_{f} t-u_{1}\right|_{X^{\alpha}} \rightarrow 0$ quando $t \rightarrow \omega_{u_{0}}^{-}$. Cauchy

Suponhamos que nossa afirmativa seja verdadeira, e consideremos o problema de

$$
\left\{\begin{array}{l}
\dot{u}+A u=f(u) \\
u\left(\omega_{u}\right)=u
\end{array}\right.
$$

O Teorema $3.3 .3 \mathrm{em}$ [15] implica que existe um $\eta>0$ tal que o problema de Cauchy (1.3) possui uma única solução em $\left[\omega_{u_{0}}, \omega_{u_{0}}+\eta\right)$. Portanto, $u_{1} \pi_{f} t$ está definido para todo $l \in\left[\omega_{\text {non }}, \omega_{\text {no }}+\eta\right)$. Definimos a seguinte função:

$$
u(t):= \begin{cases}u_{0} \pi_{f} t & \text { se } 0<t<\omega_{u_{0}} \\ u_{1} & \text { se } t=\omega_{u_{0}} \\ u_{1} \pi_{f} t & \text { se } \omega_{u_{0}}<t<\omega_{u_{0}}+\eta\end{cases}
$$

Nossa afirmação implica que $t \mapsto u(t)$ é contínua em $\left[0, \omega_{u_{0}}+\eta\right)$. Além disso, para todo $t \in\left[0, \omega_{u 0}\right]$ temos que

$$
u(l)=e^{-A t} u_{0}+\int_{0}^{l} e^{-A(t-s)} \int\left(u_{0} \pi_{f} s\right) \mathrm{d} s,
$$

e para todo $t \in\left[\omega_{u_{0}}, \omega_{u_{0}}+\eta\right]$

$$
u(t)=e^{-A t_{1}} u_{1}+\int_{\omega_{u_{u_{0}}}}^{t} e^{-A(t-s)} f\left(u_{1} \pi_{f} s\right) \mathrm{d} s
$$


Combinando (1.4) e (1.5) temos que, para todo $t \in\left[0, \omega_{u_{0}}+\eta\right)$

$$
u(t)=e^{-A t} u_{0}+\int_{0}^{t} e^{-A(t-s)} f(u(s)) \mathrm{d} s
$$

Assim, pelo Teorema 4.3 .3 de [6], temos que $t \mapsto u(t)$ é uma solução do problema de Cauchy

$$
\left\{\begin{array}{l}
\dot{u}+A u=f(u) \\
u(0)=u_{0} .
\end{array}\right.
$$

em $\left[0, \omega_{u_{0}}+\eta\right)$. Mas isso contradiz o fato de $\left[0, \omega_{u_{0}}\right)$ ser o intervalo maximal de existência da solução passando por $u_{0}$.

Assim, para completar a prova do teorema, basta mostrar que nossa afirmação é verdadeira. Como $A$ é um operador setorial con resolvente compacto existe um $k>0$ e um $\delta>0$ tal que $\sigma(A+k I)>\delta>0$. Sem perda de generalidade, podemos assumir que $|u|_{X^{\alpha}}=\left|A_{1}^{\alpha} u\right|_{X}$, onde $A_{1}=A+k I$. Notemos que u é solução de

$$
\left\{\begin{array}{l}
\dot{u}+A_{1} u=f(u)+k u, \\
u(0)=u_{0},
\end{array}\right.
$$

se, e somente se, $u$ é solução de 1.2 .

Definimos $f_{1}(u)=f(u)+k u$. Notemos que $f_{1}(N)$ é um conjunto limitado.

Para demonstrar nossa afirmação, mostremos inicialmente que o conjunto $\left\{\left|u_{0} \pi_{f}\right|_{x^{3}} \mid\right.$ $\left.\tau_{0} \leq t \leq \omega_{u_{0}}\right\}$ é limitado, onde $\tau_{0}>0$ é uma constante positiva e $\beta$ é um número real tal que $\alpha \leq \beta<1$.

Se $t \in\left[0, \omega_{u_{0}}\right)$, pela fórmula da variação das constantes temos:

$$
u_{0} \bar{u}_{f} t=e^{-\lambda_{1} t} u_{0}+\int_{J_{0}}^{t} e^{-A_{1}(t-s)} J_{1}\left(u_{0} \pi_{f} s\right) \mathrm{d} s .
$$

Logo, para $0 \leq t<\omega_{u_{0}}$, segue do Teorema 1.4 .3 de [15] que

$$
\begin{aligned}
\left|u_{0} \pi_{f} t\right|_{X^{\beta}} & \leq\left|A_{1}^{\beta-\alpha} e^{-A_{1} t} A_{1}^{\alpha} u_{0}\right|_{X^{\beta}}+\int_{0}^{t}\left|A_{1}^{\beta} e^{-A_{1}(t-s)} f_{1}\left(u_{0} \pi_{f} s\right)\right|_{X} \mathrm{~d} s \\
& \leq\left|A_{1}^{\beta-\alpha} e^{-A_{1} t}\right|\left|u_{0}\right|_{X^{\alpha}}+\int_{0}^{t}\left|A_{1}^{\beta} e^{-A_{1}(t-s)}\right|\left|f_{1}\left(u_{0} \pi_{f} s\right)\right|_{X} \mathrm{~d} s \\
& \leq C_{\beta-\alpha} t^{-(\beta-\alpha)}\left|u_{0}\right|_{X^{\alpha}}+C \int_{0}^{\iota}(t-s)^{-\beta} \mathrm{d} s
\end{aligned}
$$

onde $C=C_{\beta} \sup \left\{\left|f_{1}(u)\right|_{X} \mid u \in N\right\}$ e $C_{\beta-\alpha}$ e $C_{\beta}$ são constantes positivas como no Teorema 1.4 .3 de $[15]$. Mas

$$
\int_{0}^{l}(t-s)^{-3} \mathrm{~d} s=\frac{t^{1-\beta}}{1-\beta}
$$

Assim,

$$
\left|u_{0} \pi_{f} l\right|_{X^{\beta}} \leq C_{\beta-\alpha} t^{-(\beta-\alpha)}\left|u_{0}\right|_{X^{\alpha}}+C \frac{t^{1-\beta}}{1-\beta} .
$$


A expressão acima é limitada quando $t \rightarrow \omega_{u_{\nu}}^{-}$, isto é, existe um $C^{\prime}>0$ tal que

$$
\left|u_{0} \pi_{f} t\right|_{X^{\beta}} \leq C^{\prime} \text { para todo } t \in\left[\tau_{0}, \omega_{u_{0}}\right] \text {. }
$$

Como $X^{\alpha}$ é um espaço normado completo, para completar a demonstração basta mostrar que, para todo $\varepsilon>0$, existe um $\delta>0$ tal que. para quaisquer que sejam $t, \tau \in\left[\tau_{0}, \omega_{1 \nu_{0}}\right)$ com $|t-\tau|<\delta$, então $\left|u_{0} \pi_{f} t-u_{0} \pi_{f} \tau\right|_{x^{\alpha}}<\Xi$.

Primeiramente notemos, que se $0 \leq \tau<t<\omega_{u_{0}}$, então

$$
\begin{aligned}
u_{0} \pi_{f} l-u_{0} \pi_{f} \tau & =e^{-A_{1} t} u_{0}-u_{0} \pi_{f} \tau+\int_{0}^{\tau} e^{-A_{1}(t-s)} j_{1}\left(u_{0} \pi_{f} s\right) \mathrm{d} s+\int_{\tau}^{\tau} e^{-A_{1}(t-s)} j_{1}\left(u_{0} \pi_{f} s\right) \mathrm{d} s \\
& =e^{-A_{1}(l-\tau)} e^{-A_{1} \tau} u_{0}-u_{0} \pi_{f} \tau+\int_{0}^{\tau} e^{-A_{1}(l-\tau)} e^{-\Lambda_{1}(\tau-s)} f_{1}\left(u_{0} \pi_{f} s\right) \mathrm{d} s \\
& +\int_{\tau}^{t} e^{-A_{1}(t-s)} f_{1}\left(u_{0} \pi_{f} s\right) \mathrm{d} s \\
& =\left(e^{-A_{1}(t-\tau)}-I\right) u_{0} \pi_{f} \tau+\int_{\tau}^{t} e^{-A_{1}(t-s)} j_{1}\left(u_{0} \pi_{f} s\right) \mathrm{d} s .
\end{aligned}
$$

Pelo Teorema 1.4 .3 de [15] temos que

$$
\begin{aligned}
& \left|u_{0} \pi_{f} t-u_{0} \pi_{f} \tau\right|_{X^{\alpha}} \leq\left|A_{1}^{\alpha}\left(e^{-A_{1}(t-\tau)}-I\right) u_{0} \pi_{f} \tau\right|_{X}+\int_{\tau}^{\iota}\left|A_{1}^{\alpha x} e^{-A_{1}(t-s)} f_{1}\left(u_{0} \pi_{f} s\right)\right|_{X} \mathrm{~d} s \\
& \leq\left|\left(e^{\left.-\operatorname{ri}_{1} i-i\right)}-I\right) A_{1}^{\alpha} u_{0}{ }^{\pi} \tau\right|_{X}+C \int_{\tau}^{t}(t-s)^{\alpha} \mathrm{d} s \\
& \leq C_{\beta-\alpha}(t-\tau)^{\beta-\alpha}\left|A_{1}^{\beta-\alpha} \cdot A_{1}^{\alpha} u_{0} \pi_{f} \tau\right|_{X}-C \frac{(t-\tau)^{1-\alpha}}{1-\alpha} \\
& =C_{\beta-\alpha}(t-\tau)^{\beta-\alpha}\left|u_{0} \pi_{f} \tau\right|_{X^{\beta}}-C \frac{(t-\tau)^{1-\alpha}}{1-\alpha} \\
& \leq C_{\beta-n} C^{\prime}(t-\tau)^{\beta-\alpha} \text {. }
\end{aligned}
$$

A continuidade uniforme da função $x \mapsto x^{\beta-\alpha}$ para $x$ em um intervalo limitado compacto que, para cada $\varepsilon>0$, existe um $\delta>0$ tal que $\left|u_{0} \pi_{f} t-u_{0} \pi_{f} \tau\right|_{X^{\alpha}} \leq \varepsilon$ sempre que $|t-\tau|<\delta$. Logo, existe um $u_{1} \in X^{\alpha}$ tal que $\left|u_{0} \pi_{f} t-u_{1}\right|_{X^{\alpha}} \rightarrow 0$ quando $t \rightarrow \omega_{u_{0}}$ e a demonstração está completa

\subsection{Atratores globais}

Nessa seção vamos apresentar o conceito de atrator global associado a uma equação de evolução parabólica. Para tanto, vamos supor que $X$ seja um espaço de Banach com norma $|\cdot|_{X}$ e que $A: D(A) \subset X \longrightarrow X$ seja um operador setorial com resolvente compacto. Dado $\alpha \in[0,1)$, seja $f: X^{\alpha} \longrightarrow X$ uma função localmente Lipschitziana e suponhamos que $f$ aplica subconjuntos limitados de $X^{\alpha}$ em subconjuntos limitados de $X$. 
Seja $\pi_{f}$ o semifluxo local em $X^{\alpha}$ gerado pelas soluções da equação parabólica semilincar

$$
\dot{u}+A u=f(u) .
$$

Dizemos que $\pi_{f}$ é do tipo gradiente com relação a uma função contínua $\mathcal{L}: X \longrightarrow \mathbb{R}$, ou que $\mathcal{L}$ é uma função de Lyapunov para $\pi_{f}$ se, para toda solução não-constante $\sigma: J \longrightarrow X$ de $\pi_{f}$, a função a função $t \mapsto \mathcal{L}(\sigma(t)), t \in J$, ć cstritamente decrescente.

O Lema 1.2 .5 implica no seguinte resultado:

Teorema 1.3.1 Seja $\pi_{f}$ como acima e suponhamos que $\pi_{f}$ seja do tipo gradiente com relação a uma função $\mathcal{L}: X \longrightarrow \mathbb{R}$. Além dis.so, suponhamos que exista um $k \in[0, \infty)$ tal que

$$
|u|_{X^{\alpha}} \leq k(\mathcal{L}(u)+1) \text { qualquer que seja } u \in X^{\alpha} .
$$

Então $\pi_{f}$ é um semifluxo global.

Demonstração. Seja $u \in X^{a}$ e $\omega_{u} \in(0, \infty]$ tais que, para todo $l \geq 0, u \pi_{f} l$ está definido se, e somente se, $t \in\left[0, \omega_{u}\right)$. Nossa hipótese implica que

$$
\left|u \pi_{f} l\right|_{X^{\alpha}} \leq h\left(\mathcal{L}\left(u \pi_{f} l\right)+1\right) \leq h(\mathcal{L}(u)+1)=: h^{\prime} \text { para todo } t \in\left[0, \omega_{u}\right) .
$$

Seja $N:=\left\{\left.u \in X^{\alpha}|| u\right|_{X^{\alpha}} \leq k^{\prime}\right\}$. Logo, $N$ é limitado o fechado cm $X^{\alpha}$ e portanto, $N$ é limitado em $X$. Além disso, $u \pi_{f}\left[0, \omega_{u}\right) \subset N$ e $\pi_{f}$ não explode em $N$ pelo Lema 1.2.5. Logo, $\omega_{u}=\infty$ para todo $u \in X^{\alpha}$.

Seja $\pi_{f}$ do tipo gradiente. Dizemos que $u \in X^{\alpha}$ é um ponto de equilibrio de $\pi_{f}$ se $u \pi_{f} t=u$ para todo $t \in[0, \infty)$.

Teorema 1.3.2 Suponhamos que as hipóteses do Teorema 1.3.1 estejam satisfeitas. Além disso, suponhamos que, para todo subconjunto limitado $B$ de $X^{\alpha}$,

$$
\sup _{u \in B} \mathcal{L}(u)<\infty .
$$

Suponhamos ainda que o conjunto $E$ dos pontos de equalibrio de $\pi_{f}$ seja limitado em $X^{\alpha}$.

Seja $\mathcal{A}_{\pi_{f}}$ o conjunto de todas as órbitas completas e limitadas de $\pi_{f}$. isto é, o conjunto de todos os $u \in X^{\alpha}$ para os quais existe uma soluçâa completa $\sigma: \mathbb{R} \rightarrow X^{\alpha}$ de $\pi_{f}$ com $\sigma(0)=u$ e $\sup _{t \in \mathbb{R}}|\sigma(t)|_{X^{\alpha}}<\infty$. As seguintes afirmativas são válidas:

(1) $\pi_{f}$ é um semifluxo global;

(2) $\mathcal{A}_{\pi,}$ é näo-vazio, compacto, conexo em $X^{\alpha}$ e atrai todo conjunto limitado $B$ de $X^{\alpha}$, isto é, dado $\delta>0$, existe um $t(B, \delta)>0$ tal que

$$
\inf _{v \in \mathcal{A}_{\pi_{f}}}\left|u \pi_{f} t-v\right|_{X^{\alpha}}<\delta \text { para todo } u \in B \text { e todo } t \geq t(B, \delta) .
$$


O conjunto $\mathcal{A}_{\pi_{f}}$ é chamado atrator global de $\pi_{f}$

Notemos que a afirmativa (1) do Teorema 1.3.2 segue do Teorema 1.3.1. Para demonstrarmos a afirmativa (2) utilizamos o Tcorcma 3.2 de [16]. Assim, precisamos mostrar que $\pi_{f}$ é limitado e assintoticamente compacto (no sentido de [16]). Os próximos lemas demonstram esses fatos. Vamos então assumir as hipóteses do Teorema 1.3.2.

Lema 1.3.3 O semifluxo $\pi_{f}$ é limitado, isto é, se $B$ é um subconjunto limitado em $X^{\alpha}$, então $B \pi_{f}[0, \infty)$ é limitado em $X^{\alpha}$.

Demonstração. Suponhamos que $B$ seja um subconjunto limitado em $X^{\alpha}$ e sejam $u \in B$ e $t \geq 0$. Portanto,

$$
\left|u \pi_{f} t\right|_{X^{\alpha}} \leq k\left(\mathcal{L}\left(u \pi_{f} t\right)+1\right) \leq k(\mathcal{L}(u)+1) \leq k\left(\sup _{u \in B} \mathcal{L}(u)+1\right)=h^{\prime \prime} \in(0, \infty)
$$

Logo,

$$
\left|u \pi_{f} t\right|_{X^{\alpha}} \leq k_{i}^{\prime \prime} \text { para todo } u \in B \text { e todo } t \geq 0
$$

A demonstração do lema está concluída.

O próximo lema nos auxiliará na demonstração de que $\pi_{f}$ é assintoticamente compacto.

Lema 1.3.4 Seja $N \subset X^{\mathrm{u}}$ um subconjunto limitado e fechado em $X^{\alpha}$. Seja $\varepsilon>0$ arbitrário. Então existe um conjunto compacto $C=C(N, \Xi) \subset X^{\alpha}$ tal que, sempre que $u \pi_{f} t \in N$ para todo $t \in\left[0, t_{0}\right)$, para algum $t_{0} \in(0, \infty]$, segue que $u \pi_{f} t \in C$ para todo $t \in\left[\hat{\varepsilon}, t_{0}\right)$.

Demonstração. Seja $\beta \in \mathbb{R}$ tal que $\alpha<\beta<1$. Como $N$ é um subconjunto fechado e limitado em $X^{\alpha}$, temos que $f(N)$ e limitado $\mathrm{cm} X$. Portanto, existe um $L>0$ tal que

$$
|f(u)|_{X} \leq L \text { para todo } x \in N .
$$

Mostremos que existe um $b=b(N, \varepsilon)>0$ tal que, sempre que $u \pi_{f} t \in N$ para todo $\iota \in\left[0, \iota_{0}\right)$, para algum $\iota_{0} \in(0, \infty]$, então $\left|u \pi_{f}\right|_{X^{3}} \leq b$ para todo $\iota \in\left[\bar{\varepsilon}, \iota_{0}\right)$. Como $A$ é um operador setorial, existe um $k>0$ tal que $\sigma(A+k I)>\delta>0$. Vamos assumir que $|u|_{X^{\alpha}}=\left|A_{1}^{\wedge} u\right|_{X}$, onde $A_{1}=A+k I$ e $f_{1}(u)=f(u)+k u$, como na prova do Lema 1.2.5. Então, pela Fórmula da Variação das Constantes:

$$
u \pi_{f} t=e^{-A_{1} t} u+\int_{0}^{t} e^{-A_{\mathbf{1}}(i-s)} f_{1}\left(u \pi_{f} s\right) \mathrm{d} s \text { para } t>0 .
$$

Assim, para $0<t<t_{0}$, segue do Teorema 1.4 .3 de [15] que

$$
\begin{aligned}
\left|u \pi_{f} t\right|_{X^{\beta}} & =\left|A_{1}^{3} e^{-A_{1} t} u+\int_{0}^{t} A_{1}^{\beta \beta} e^{-A_{1}(t-s)} f_{1}\left(u \pi_{f} s\right) \mathrm{d} s\right|_{X} \\
& \leq\left|A_{1}^{\beta} e^{-A_{1} t}\right||u|_{X}+\int_{0}^{t}\left|A_{1}^{\beta} e^{-A_{1}(t-s)}\right|\left|f_{1}\left(u \pi_{f} s\right)\right|_{X} \mathrm{~d} s \\
& \leq C_{\beta} t^{-\beta} e^{-\delta t} L_{1}+\int_{0}^{\iota} C_{\beta}(t-s)^{-\beta} e^{-\omega \delta(t-s)}\left(L+k L_{1}\right) \mathrm{d} s,
\end{aligned}
$$


onde $L_{1}=\sup \left\{|u|_{X} \mid u \in N\right\}$. Portanto, para todo $t \in\left[\varepsilon, t_{0}\right)$ temos que, fazendo $t-s-z ;$

$$
\left\lceil\left. u \pi_{f} t\right|_{X^{\beta}} \leq C_{\beta} L_{1} \varepsilon^{-\beta} e^{-\delta \varepsilon}+C_{\beta}\left(L+k L_{1}\right) \int_{U}^{l} z^{-\beta} e^{-\delta z} \mathrm{~d} z .\right.
$$

Notemos que,

$$
\begin{aligned}
\int_{0}^{t} z^{-\beta} e^{-\delta z} \mathrm{~d} z & =\int_{J_{0}}^{\varepsilon} z^{-\beta} e^{-\delta z} \mathrm{~d} z+\int_{J_{\varepsilon}}^{l} z^{-\beta} e^{-\delta z} \mathrm{~d} z \\
& \leq \int_{0}^{e} z^{-\beta} e^{-\delta z} \mathrm{~d} z+\int_{=}^{\infty} z^{-\beta} e^{-\delta z} \mathrm{~d} z
\end{aligned}
$$

Como $z^{\beta}=e^{-\beta \ln z} \leq \varepsilon^{-\beta}$, obtemos

$$
\begin{aligned}
\int_{0}^{l} z^{-\beta} e^{-\delta z} \mathrm{~d} z & \leq \int_{0}^{\varepsilon} z^{-\beta} e^{-\delta z} \mathrm{~d} z+\varepsilon^{-\beta} \int_{\varepsilon}^{\infty} e^{-\delta z} \mathrm{~d} z \\
& \leq \int_{3}^{\varepsilon} z^{-\beta} \mathrm{d} z+\varepsilon^{-\beta} \int_{\varepsilon}^{\infty} e^{-\delta z} \mathrm{~d} z=: M(\varepsilon, \delta)<\infty .
\end{aligned}
$$

Substituindo em (1.6) temos que

$$
\left|u_{0} \pi_{f} t\right|_{X^{\beta}} \leq b \text { para todo } t \in\left[\hat{\varepsilon}, t_{0}\right),
$$

onde $b:=C_{\beta} L_{1} \varepsilon^{-\beta} e^{-\delta \varepsilon}+C_{\beta}\left(L+h L_{1}\right) M(\varepsilon, \delta)$. Isso completa a primeira parte da demonstração.

Para completar a demonstração recordemos que, como $A$ possui resolvente compacto, a inclusão $X^{\beta} \hookrightarrow X^{\alpha}$ é compacta para $\alpha<\beta<1$. Consideremos $C:=\left\{\left.u \in X^{\alpha}|| u\right|_{X^{\beta}} \leq\right.$ b\}. Segue que $C$ é um conjunto compacto na topologia de $X^{a}$ e (1.7) implica que $u_{0} \pi_{f} t \in C$ para todo $t \in\left[c, t_{0}\right)$.

Lema 1.3.5 O semifluxo $\pi_{f}$ é assintoticamente compacto, isto é, se $B$ é um subconjunto limitado de $X^{\alpha}$ tal que $B \pi_{f}[\tau, \infty)$ é limitado para algum $\tau \geq 0$, então todo conjunto da forma $\left\{u_{n} \pi_{f} t_{n} \mid n \in \mathbb{N}\right\}$ com $u_{n} \in B, t_{n} \in[0, \infty)$ para todo $n \in \mathbb{N}$ e $t_{n} \rightarrow \infty$ quando $n \rightarrow \infty$ é relativamente compacto.

Demonstração. Seja $B$ um subconjunto limitado de $X^{\alpha}$ tal que existe um $\tau \geq 0$ com $B \pi_{f}[\tau, \infty)$ limitado. Consideremos sequências $\left(u_{n}\right)_{n \in \mathbb{N}}$ em $B$ e $\left(t_{n}\right)_{n \in \mathbb{N}}$ en $[0, \infty)$ com $t_{n} \rightarrow \infty$ quando $n \rightarrow \infty$. Por hipótese, existe um $L>0$ tal que

$$
\left|u_{n} \pi_{f} t\right|_{X^{\circ}} \leq L \text { para todo } t \in[\tau, \infty] \text { e todo } n \in \mathbb{N} .
$$

Como $t_{n} \rightarrow \infty$, podemos assumir que $t_{n} \geq \tau=: \varepsilon$ para todo $n \in \mathbb{N}$. Definamos $N:=$ $\left\{\left.u \in X^{\alpha}|| u\right|_{X^{\alpha}} \leq L\right\}$. Pelo Lema 1.3.4 existe um conjunto compacto $C \subset X^{\alpha}$ tal que

$$
u_{n} \pi_{f}\left[\tau, t_{n}\right] \subset C \text { para todo } n \in \mathbb{N}
$$


Em particular, a sequência $\left(u_{n} \pi_{f} t_{n}\right)_{n \in \mathbb{N}}$ possui uma subsequência convergente.

Demonstração do Teorema 1.3.2. As hipóteses sobre $f$, os Lemas 1.3 .3 e 1.3 .5 e as hipótcses sobrc $\pi_{f}$ implicam que as hipóteses do Teorema 2.3 de [16] estão satisfeitas. Logo, obtemos as conclusões do teorema.

Observação 1.3.6 Como é rcssaltado cm [22] na Proposição 2.15, o conceito de semifluxo assintoticamente compacto apresentado aqui e definido por Ladyzhenskaya em [16] ¿ equivalente ao conceito de semifluxo assintoticamente suave, apresentado por Hale cm [13]. 


\section{Capítulo 2}

\section{As Equações de Reação-Difusão em Domínios Finos}

Iniciamos esse capítulo apresentando a relação entre os problemas $\left(\tilde{E}_{\varepsilon}\right)$ e $\left(E_{\varepsilon}\right)$ apresentados na Introdução. A equivalência obtida nos permite estudar a equação de evolução abstrata associada a $\left(\Gamma_{\varepsilon}\right)$. () passo seguinte é definir o problema limite. Na Scção 2.2 apresentamos os "espaços limites" no qual a equação de evolução abstrata estará definida. Finalizamos o capítulo apresentando o problema limite para a família $\left(E_{\varepsilon}\right), \varepsilon>0$.

\subsection{As equações de reação-difusão em domínios finos}

Sejam $M$ c $N$ inteiros positivos. Identificaremos $\mathbb{R}^{M+N} \operatorname{com} \mathbb{R}^{M} \times \mathbb{R}^{N}$ e escreveremos $(x, y)$ para um ponto genérico de $\mathbb{R}^{N+N}$. Seja $\Omega \subset \mathbb{R}^{M+N}$ um domínio limitado não-vazio e com fronteira Lipschitz. Dado $\varepsilon>0$, consideremos a transformação de compressão $T_{\xi}: \mathbb{R}^{M+N} \longrightarrow \mathbb{R}^{M+N}$ dada por $T_{\varepsilon}(x, y)=(x, \varepsilon y)$. Definimos $\Omega_{\varepsilon}:=T_{\varepsilon}(\Omega)$. Geometricamente, $T_{\varepsilon}$ comprime o domínio $\Omega$ na direção $y$ pelo fator $\varepsilon$.

Consideremos a seguinte equação de reação-difusão em $\Omega_{\varepsilon}$ com condições de fronteira do tipo Neumann:

$$
\begin{array}{ll}
u_{t}=\Delta u+f(u), & t>0,(x, y) \in \Omega_{\bar{\varepsilon}}, \\
\partial_{\nu_{\varepsilon}} u=0, & t>0,(x, y) \in \partial \Omega_{\varepsilon},
\end{array}
$$

onde $\nu_{\varepsilon}$ é a normal exterior em $\partial \Omega_{\varepsilon}$ e $f: \mathbb{R} \longrightarrow \mathbb{R}$ é uma não-linearidade que satisfaz certas condições de crescimento e dissipatividade as quais iremos caracterizar mais tarde.

Nosso interesse é analisar o que ocorre com a dinâmica de $\left(\tilde{E}_{\varepsilon}\right)$ quando $\varepsilon \rightarrow 0^{+}$. Nossa hipótese de dissipatividade sobre $f$ implicará que, para cada $\varepsilon>0$, existe um atrator global $\tilde{\mathcal{A}}_{\pi_{\varepsilon}}$ para $\left(\tilde{E}_{\varepsilon}\right)$. Assim, discutiremos o que ocorre com a família de atratores $\left(\mathcal{A}_{\text {Tr }}\right)_{\varepsilon>0}$ quando $\varepsilon \rightarrow 0^{+}$. Entretanto, temos a seguinte dificuldade técnica: o parâmetro $\varepsilon$ aparece no domínio $\Omega_{\varepsilon}$ de cada equação $\left(\tilde{E}_{\varepsilon}\right)$. O primeiro passo, então, será obter um problema $\left(E_{z}\right)$ equivalente a $\left(\tilde{E}_{\varepsilon}\right)$ no qual o domínio é $\Omega$. para todo $\varepsilon>0$. 
Fixado $\varepsilon>0$, para cada função $u: \Omega_{\bar{\Xi}} \longrightarrow \mathbb{R}$, definimos a aplicação $\Phi_{\varepsilon}(u):=u \circ T_{\varepsilon}$. É claro que $\Phi_{\varepsilon}$ é uma transformação linear. Além disso, vale o seguinte resultado:

Lema 2.1.1 Seja $\varepsilon>0$. As restrições $\Phi_{\varepsilon}: \mathcal{D}\left(\Omega_{\Sigma}\right) \longrightarrow \mathcal{D}(\Omega), \Phi_{\varepsilon}: H^{1}\left(\Omega_{\varepsilon}\right) \longrightarrow H^{1}(\Omega)$ e $\Phi_{\varepsilon}: L^{2}\left(\Omega_{\varepsilon}\right) \longrightarrow L^{2}(\Omega)$ estão bem definidas e são isomorfismos lineares.

Demonstração. Seja $\varepsilon>0$. Mostremos que $\Phi_{\varepsilon}\left(L^{2}\left(\Omega_{\varepsilon}\right)\right) \subset L^{2}(\Omega)$. Dado $u \in L^{2}\left(\Omega_{\varepsilon}\right)$, pelo Teorema de Mudança de Variáveis temos que

$$
\begin{aligned}
\int_{\Omega 2}\left|\Phi_{\varepsilon}(u)(x, y)\right|^{2} \mathrm{~d} x \mathrm{~d} y & =\int_{\Omega}\left|u \circ T_{\varepsilon}(x, y)\right|^{2} \mathrm{~d} x \mathrm{~d} y=\left|\operatorname{det}\left[T_{\varepsilon}\right]\right|^{-1} \int_{\Omega 2}\left|u \circ T_{\varepsilon}(x, y)\right|^{2}\left|\operatorname{det}\left[T_{\varepsilon}\right]\right| \mathrm{d} x \mathrm{~d} y \\
& =\left|\operatorname{det}\left[T_{\xi}^{\prime}\right]\right|^{-1} \int_{\Omega_{\varepsilon}}|u(x, \Xi y)|^{2} \mathrm{~d} x \mathrm{~d} y=\left|\operatorname{det}\left[T_{\varepsilon}\right]\right|^{-1}|u|_{L^{2}\left(\Omega_{\varepsilon}\right)}^{2},
\end{aligned}
$$

onde $\left[T_{\varepsilon}\right]$ é a matriz de $T_{\varepsilon}$ em relação à base canônica de $\mathbb{R}^{M+N}$. Segue que

$$
\left|\Phi_{\varepsilon}(u)\right|_{L^{2}(\Omega)}^{2}=\varepsilon^{-N}|u|_{L^{2}\left(\Omega_{\varepsilon}\right)}^{2} \text {. }
$$

Portanto, $\Phi_{\varepsilon}(u) \in L^{2}(\Omega)$, isto é, $\Phi_{\varepsilon}: L^{2}\left(\Omega_{\varepsilon}\right) \longrightarrow L^{2}(\Omega)$ está bem definido. Por outro lado, dada uma função $u \in L^{2}(\Omega)$, consideremos $v:=u \circ T_{\varepsilon^{-1}}$ e notemos que

$$
\begin{aligned}
\int_{\Omega_{\varepsilon}}|v(x, \varepsilon y)|^{2} \mathrm{~d} x \mathrm{~d} y & =\int_{\Omega_{\varepsilon}}\left|u \circ T_{\varepsilon^{-1}}(x, \varepsilon y)\right|^{2} \mathrm{~d} x \mathrm{~d} y \\
& =\left|\operatorname{det}\left[T_{\varepsilon^{-1}}\right]\right|^{-1} \int_{\Omega_{\varepsilon}}\left|u \circ T_{\varepsilon^{-1}}(x, \varepsilon y)\right|^{2}\left|\operatorname{det}\left[T_{\varepsilon^{-11}}^{\prime}\right]\right| \mathrm{d} x \mathrm{~d} y \\
& =\left|\operatorname{det}\left[T_{\varepsilon^{-1}}\right]\right|^{-1} \int_{\Omega 2}|u(x, y)|^{2} \mathrm{~d} x \mathrm{~d} y=\varepsilon^{N}|u|_{L^{2}(\Omega)}^{2},
\end{aligned}
$$

isto é, $v \in L^{2}\left(\Omega_{\varepsilon}\right)$. Também, $\Phi_{\varepsilon}(v)=\left(u \circ T_{\varepsilon^{-1}}\right) \circ T_{\varepsilon}=u$. Sendo assim, $\Phi_{\varepsilon}\left(L^{2}\left(\Omega_{\varepsilon}\right)\right)=$ $I_{2}^{2}(\Omega)$

Seja $v \in L^{2}\left(\Omega_{\varepsilon}\right)$ tal que $\Phi_{\varepsilon}(v)=0$. Segue de $(2.1)$ que $v=0$. Como $\Phi_{\varepsilon}$ é linear, temos que $\Phi_{\varepsilon}$ é injetora e, portanto, um isomorfismo.

Consideremos agora o caso $\Phi_{\varepsilon}: \mathcal{D}\left(\Omega_{\varepsilon}\right) \longrightarrow \mathcal{D}(\Omega)$. É claro que, se $\varphi \in \mathcal{D}\left(\Omega_{\varepsilon}\right)$, então $\varphi \circ T_{\varepsilon} \in C^{\infty}(\Omega)$. Verifiquemos que $\varphi \circ T_{\varepsilon}$ tem suporte compacto contido em $\Omega$. Para isto, consideremos o conjunto $A:-T_{\varepsilon^{-1}}(\operatorname{supp} \varphi)$. A continuidade de $T_{\varepsilon^{-1}}$ implica que $A$ ó compacto e além disso, $A \subset \Omega$. Tomando $\left(x_{0}, y_{0}\right) \in \Omega \backslash A$, temos que existe uma vizinhança aberta $U$ de $\left(x_{0}, y_{0}\right)$ com $U \subset \Omega \backslash A$. Observemos também que $T_{\varepsilon}(U) \subset \Omega_{\varepsilon} \backslash \operatorname{supp} \varphi$. Portanto, $\varphi \circ T_{\varepsilon}(x, y)=0$, para todo $(x, y) \in U$. Com isso, supp $\varphi \circ T_{\varepsilon} \subset A$ e assim, $\varphi \circ T_{\varepsilon}$ possui suporte compacto em $\Omega$, ou seja, $\Phi_{\varepsilon}: \mathcal{D}\left(\Omega_{\varepsilon}\right) \longrightarrow \mathcal{D}(\Omega)$ está bem definido. Agora, dada uma função $\varphi \in \mathcal{D}(\Omega)$, considcramos a função $\phi:=\varphi \circ T_{\varepsilon^{-1}} \mathrm{c}$ um raciocínio análogo ao acima implica que $\phi \in \mathcal{D}\left(\Omega_{\varepsilon}\right)$. Também, se $\Psi_{\varepsilon}(\varphi)=0$ temos que $\varphi=0 \mathrm{e}$ assim, $\Phi_{\varepsilon}: \mathcal{D}\left(\Omega_{\xi}\right) \longrightarrow \mathcal{D}(\Omega)$ é um isomorfismo.

Seja $v \in I^{1}\left(\Omega_{\varepsilon}\right)$. Então $v \circ T_{\varepsilon} \in L^{2}(\Omega), \partial_{x_{i}} v \circ T_{\varepsilon} \in L_{i}^{2}(\Omega), i=1, \ldots, M$, e $\partial_{y_{j}} v \circ T_{\varepsilon} \in$ $L^{2}(\Omega), j=1, \ldots, N$. Para cada $\varphi \in \mathcal{D}\left(\Omega_{\varepsilon}\right)$,

$$
\nabla\left(\varphi \circ T_{\varepsilon}\right)=\left(\partial_{x_{i}} \varphi \circ T_{\varepsilon}, \ldots, \partial_{x_{M}} \varphi \circ T_{\varepsilon}, \varepsilon \partial_{y_{1}} \varphi \circ T_{\varepsilon}, \ldots, \varepsilon \partial_{y_{N}} \varphi \circ T_{\varepsilon}^{\prime}\right) .
$$


Assim, para cada $i=1, \ldots, M$, usando novamente o Teorema de Mudança de Variáveis encontramos

$$
\int_{\Omega}\left(v \circ T_{\varepsilon}\right) \partial_{x_{i}}\left(\varphi \circ T_{\varepsilon}\right) \mathrm{d} x \mathrm{~d} y=-\int_{\Omega} \partial_{x_{i}} v \circ T_{\varepsilon}\left(\varphi \circ T_{\varepsilon}\right) \mathrm{d} x \mathrm{~d} y \text { qualquer que seja } \varphi \in \mathcal{D}\left(\Omega_{\Sigma}\right) \text {. }
$$

Como $\Phi_{\varepsilon}: \mathcal{D}\left(\Omega_{\varepsilon}\right) \longrightarrow \mathcal{D}(\Omega)$ é um isomorfismo, obtemos,

$$
\int_{\Omega}^{n}\left(v \circ T_{\varepsilon}\right) \partial_{x_{i}} \psi \mathrm{d} x \mathrm{~d} y=-\int_{\Omega}\left(\partial_{x_{i}} v \circ T_{\varepsilon}\right) \psi \mathrm{d} x \mathrm{~d} y \text { qualquer que seja } \psi \in \mathcal{D}(\Omega),
$$

isto 6 ,

$$
\partial_{x_{i}}\left(v \circ T_{\Xi}\right)=\partial_{x_{i}} v \circ T_{\varepsilon}, \text { para } i=1, \ldots, M .
$$

Analogamente mostramos que

$$
\partial_{y_{j}}\left(v \circ T_{\varepsilon}\right)=\varepsilon \partial_{y_{j}} v \circ T_{\Sigma}, \operatorname{para} j=1, \ldots, N
$$

Procedendo como anteriormente mostramos que $\Phi_{\varepsilon}: H^{1}\left(\Omega_{\varepsilon}\right) \longrightarrow H^{1}(\Omega)$ é um isomorfismo.

Para cada $\varepsilon>0$, a aplicação $\check{\Psi}_{\varepsilon}$ nos auxiliará na construção de um problema $\left(E_{\varepsilon}\right)$ que será conjugado ao probloma $\left(E_{\varepsilon}\right)$. Para isso, comeşaremos com a definição das formas bilineares associadas a estes problemas.

Dado $\varepsilon>0$, definimos as formas bilineares $\tilde{a}_{\varepsilon}: H^{1}\left(\Omega_{\varepsilon}\right) \times H^{1}\left(\Omega_{\varepsilon}\right) \longrightarrow \mathbb{R}$ por

$$
\tilde{a}_{\varepsilon}(u, v):=\int_{\Omega_{\Omega}} \nabla u \cdot \nabla v \mathrm{~d} x \mathrm{~d} y
$$

e $a_{\varepsilon}: H^{1}(\Omega) \times H^{1}(\Omega) \rightarrow \mathbb{R}$ por

$$
a_{\varepsilon}(u, v):=\int_{\Omega}\left(\nabla_{x} u \cdot \nabla_{x} v+\frac{1}{\varepsilon^{2}} \nabla_{y} u \cdot \nabla_{y} v\right) \mathrm{d} x \mathrm{~d} y
$$

Lema 2.1.2 Seja $\Phi_{\varepsilon}, \varepsilon>0$, como acima. Ás seguintes afirmativas são válidas:

(1) para cada $\varepsilon>0, \tilde{a}_{\varepsilon}$ e $a_{\varepsilon}$ são formas bilineares contínuas e simétricas;

(2) para quaisquer que sejam $u, v \in H^{1}\left(\Omega_{\S}\right)$,

$$
a_{\varepsilon}\left(\Phi_{\varepsilon} u, \Phi_{\varepsilon} v\right)=\frac{1}{\left|\operatorname{det}\left[T_{\varepsilon}\right]\right|} \tilde{a}_{\varepsilon}(u, v) ;
$$

(3) para quaisquer que sejam $u, v \in L^{2}\left(\Omega_{\varepsilon}\right)$,

$$
\left\langle\Phi_{\tilde{\varepsilon}} u, \Phi_{\varepsilon} v\right\rangle_{L^{2}(\Omega)}=\frac{1}{\left|\operatorname{det}\left[T_{\varepsilon}\right]\right|}\langle u, v\rangle_{L^{2}\left\{\Omega_{\varepsilon}\right\}} .
$$


Demonstração. É claro que $\tilde{a}_{\varepsilon}$ e $a_{\varepsilon}, \varepsilon>0$, são formas bilineares simétricas. Seja $\varepsilon>0$. A Desigualdade de Hölder implica que

$$
\left|\tilde{a}_{\varepsilon}(u, v)\right| \leq|u|_{H^{1}\left(\Omega_{\varepsilon}\right)}|v|_{H^{1}\left(\Omega_{\varepsilon}\right)} \text { quaisquer que sejam } u, v \in H^{1}\left(\Omega_{\varepsilon}\right) .
$$

Portanto, $\tilde{a}_{\varepsilon}$ é contínua.

Se $0<\varepsilon \leq 1$, segue que

$\left|a_{\tilde{\varepsilon}}(u, v)\right| \leq \varepsilon^{-2}|u|_{H^{1}(\Omega)}|v|_{H^{1}(\Omega)}$ quaisquer que sejam $u, v \in H^{1}(\Omega)$.

No caso $\varepsilon \geq 1$,

$$
\left|a_{\varepsilon}(u, v)\right| \leq|u|_{H^{1}(\Omega)}|v|_{H^{1}(\Omega)} \text { quaisquer que sejam } u, v \in H^{1}(\Omega) .
$$

Logo, $a_{\varepsilon}, \hat{\varepsilon}>0$, é uma forma bilinear contínua e a demonstração de (1) está completa.

Vamos demonstrar (2) e (3) segue de maneira análoga. Dadas $u, v \in H^{1}\left(\Omega_{s}\right)$, temos

$$
\begin{aligned}
a_{\varepsilon}\left(\Phi_{\varepsilon} u, \Phi_{\varepsilon} v\right) & =\int_{\Omega}\left(\nabla_{x} \Phi_{\varepsilon} u \cdot \nabla_{x} \Phi_{\varepsilon} v+\frac{1}{\varepsilon^{2}} \nabla_{y} \Phi_{\varepsilon} u \cdot \nabla_{y} \Phi_{\varepsilon} v\right) \mathrm{d} x \mathrm{~d} y \\
& =\int_{J_{\Omega}}\left(\left(\nabla_{x} u \cdot \nabla_{x} v\right) \circ T_{\varepsilon}+\frac{1}{c^{2}}\left(\nabla_{y} u \cdot \nabla_{y} v\right) \circ T_{\varepsilon}\right) \mathrm{d} x \mathrm{~d} y \\
& =\int_{\Omega}(\nabla u \cdot \nabla v) \circ T_{\varepsilon} \mathrm{d} x \mathrm{~d} y=\frac{1}{\left|\operatorname{det}\left[T_{\varepsilon}\right]\right|} \tilde{a}_{\varepsilon}(u, v),
\end{aligned}
$$

onde a última igualdade segue da demonstração do Lema 2.1.1.

Para cada $\varepsilon>0$, denotemos por $\tilde{b}_{\bar{\varepsilon}}$ a restrição de $\left\langle\cdot \dot{\prime}^{\prime}\right\rangle_{L^{2}\left(\Omega_{\varepsilon}\right)}$ a $H^{1}\left(\Omega_{\varepsilon}\right) \times H^{1}\left(\Omega_{\varepsilon}\right)$ e por $b$ a restrição de $\langle\cdot, \cdot\rangle_{L^{2}(\Omega)}$ a $H^{1}(\Omega) \times H^{1}(\Omega)$. Vale o seguinte resultado:

Lema 2.1.3 Seja $\varepsilon>0$. O par $(\lambda, u)$ é um par autovalor-autovetor de $\left(\tilde{a}_{\varepsilon}, \tilde{b}_{\varepsilon}\right)$ se, e somente se, $\left(\lambda, \Phi_{\varepsilon} u\right)$ é um par autovalor-autovetor de $\left(a_{\varepsilon}, b\right)$.

Demonstração. Se $(\lambda, u)$ é um par autovalor-autovetor de $\left(\tilde{a}_{\varepsilon}, \tilde{b}_{\varepsilon}\right)$, então $u \neq 0$ e, para qualquer que seja $v \in H^{1}\left(\Omega_{\varepsilon}\right)$, temos que $\tilde{a}_{\varepsilon}(u, v)=\lambda\langle u, v\rangle_{L^{2}\left(\Omega_{\varepsilon}\right)}$. Logo, o Lema 2.1.2 implica que

$$
\left|\operatorname{det}\left[T_{\varepsilon}\right]\right| a_{z}\left(\Phi_{\varepsilon} u, \Phi_{\varepsilon} v\right)=\tilde{a}_{\varepsilon}(u, v)=\lambda\langle u, v\rangle_{L^{2}\left(\Omega_{\varepsilon}\right)}=\lambda\left|\operatorname{det}\left[T_{\varepsilon}\right]\right|\left\langle\Phi_{\varepsilon} u, \Phi_{\varepsilon} v\right\rangle_{L^{2}(\Omega)} .
$$

Como $\Phi_{\varepsilon}: H^{1}\left(\Omega_{\varepsilon}\right) \longrightarrow H^{1}(\Omega)$ é um isomorfismo, segue que $\Phi_{\bar{\varepsilon}}(u) \neq 0$ e

$$
a_{\varepsilon}\left(\Phi_{\bar{\varepsilon}} u, w\right)=\lambda b\left(\Phi_{\varepsilon} u, w\right) \text { qualquer que seja } w \in H^{1}(\Omega) .
$$

O lema está demonstrado. 
Fixemos $\varepsilon>0$ e seja $u \in H^{1}\left(\Omega_{*}\right)$. Temos

$$
\begin{aligned}
\left|\tilde{a}_{\varepsilon}(u, u)\right| & =\left.\left|\sum_{i=1}^{M}\right| \partial_{x_{i}} u\right|_{L^{2}\left(\Omega_{\varepsilon}\right)} ^{2}+\sum_{j=1}^{N}\left|\partial_{y_{j}} u\right|_{L^{2}\left(\Omega_{\varepsilon}\right)}^{2} \mid \\
& =\sum_{i=1}^{M}\left|\partial_{x_{i}} u\right|_{L^{2}\left(\Omega_{\varepsilon}\right)}^{2}+\sum_{j=1}^{N}\left|\partial_{y_{j}} u\right|_{L^{2}\left(\Omega_{\varepsilon}\right)}^{2}+|u|_{L^{2}\left(\Omega_{\varepsilon}\right)}^{2}-|u|_{L^{2}\left(\Omega_{\varepsilon}\right)}^{2} \\
& =|u|_{H^{1}\left(\Omega_{\varepsilon}\right)}^{2}-|u|_{L^{2}\left(\Omega_{\varepsilon}\right)}^{2} .
\end{aligned}
$$

Analogamente, se $\varepsilon>0$ e $v \in H^{1}(\Omega)$, temos

$$
\left|a_{\varepsilon}(v, v)\right|=\sum_{i=1}^{M}\left|\partial_{x_{i}} v\right|_{L^{2}(\Omega)}^{2}+\frac{1}{\varepsilon^{2}} \sum_{j=1}^{N}\left|\partial_{y_{j}} v\right|_{L^{2}(\Omega)}^{2} .
$$

Se $0<\varepsilon<1$, segue que

$$
\left|a_{\varepsilon}(v, v)\right| \geq \sum_{i=1}^{M}\left|\partial_{x_{i}} v\right|_{L^{2}(\Omega)}^{2}+\sum_{j=1}^{N}\left|\partial_{y_{j}^{\prime}} v\right|_{L^{2}(\Omega)}^{\tilde{2}}=|u|_{H^{1}(\Omega)}^{2}-|u|_{L^{2}(\Omega)}^{2}
$$

Por outro lado, se $\varepsilon \geq 1$ temos que

$$
\left|a_{\varepsilon}(v, v)\right| \geq \frac{1}{\varepsilon^{2}} \sum_{i=1}^{M}\left|\partial_{x_{i}} v\right|_{L^{2}(\Omega)}^{2}+\frac{\frac{1}{\varepsilon^{2}}}{\sum_{j=1}}\left|\partial_{y_{j}} v\right|_{L^{2}(\Omega)}^{2}=\frac{1}{\varepsilon^{2}}|u|_{H^{1}(\Omega)}^{2}-\frac{1}{\varepsilon^{2}}|u|_{L^{2}(\Omega)}^{2}
$$

Assim, demonstramos o seguinte resultado:

Lema 2.1.4 Para cada $\varepsilon>0$, as formas bilineares $\tilde{a}_{\varepsilon}$ e $a_{\varepsilon}$ são coercivas.

Os Lemas 2.1.2 e 2.1.4 mostram que as hipóteses das Proposições 1.1.2 e 1.1.6 estão satisfeitas. Com isso temos:

Teorema 2.1.5 Para cada $\varepsilon>0$, valem:

(1) o par $\left(\tilde{a}_{\varepsilon}, \tilde{b}_{\xi}\right)$ gera um operador linear auto-adjunto com resolvente compacto $\tilde{\Lambda}_{\varepsilon}$ erm $\left(L^{2}\left(\Omega_{\varepsilon}\right),\langle\cdot, \cdot\rangle_{L^{2}\left(\Omega_{\varepsilon}\right)}\right)$ tal que, se $u \in D\left(\tilde{A}_{\varepsilon}\right)$,

$$
\tilde{a}_{\varepsilon}(u, v)=\left\langle\ddot{A}_{\varepsilon} u, v\right\rangle_{L^{2}\left(\Omega_{\varepsilon}\right)} \text { qualquer que seja } v \in H^{1}\left(\Omega_{*}\right) \text {. }
$$

Além disso, $D\left(A_{\varepsilon}\right)$ é um subespaço vetorial denso em $I^{1}\left(\Omega_{\varepsilon}\right)$ e em $L^{2}\left(\Omega_{\varepsilon}\right)$;

(2) o par $\left(a_{\varepsilon}, b\right)$ gera um operador linear auto-adjunto com resolvente compacto $A_{\varepsilon}$ em $\left(L^{2}(\Omega),\langle\cdot, \cdot\rangle_{L^{2}(\Omega)}\right)$ tal que, se $u \in D\left(A_{\varepsilon}\right)$,

$$
a_{\varepsilon}(u, v)=\left\langle A_{\varepsilon} u, v\right\rangle_{L^{2}(\Omega)} \text { qualquer que seja } v \in H^{1}(\Omega) .
$$

Além disso, $D\left(A_{\varepsilon}\right)$ é um subespaço vetorial denso em $H^{1}(\Omega)$ e em $L^{2}(\Omega)$. 
(3) O conjunto dos autovalores do par $\left(\tilde{a}_{\varepsilon}, \tilde{b}_{\varepsilon}\right)$ (respectivamente, $\left(a_{\varepsilon}, b_{\varepsilon}\right)$ ) é infinito e enumerável, possui um menor elemento e cada autovalor possui multiplicidade finita.

Os operadores $\tilde{A}_{\varepsilon}$ e $A_{\varepsilon}$ estão relacionados como mostra o próximo lema.

Lema 2.1.6 Fixemos $\varepsilon>0$. Sejam $\tilde{A}_{\varepsilon}$ e $A_{\varepsilon}$ como no Teorema 2.1.5. Então:

(1) $D\left(A_{\varepsilon}\right)=\Phi_{\varepsilon}\left(D\left(\hat{A}_{\varepsilon}\right)\right)$;

(2) $A_{\varepsilon}\left(\Phi_{\varepsilon} u\right)=\Phi_{\varepsilon}\left(\ddot{A}_{\varepsilon} u\right)$ qualquer que seja $u \in D\left(\tilde{A}_{\varepsilon}\right)$.

Demonstração. Seja $u \in D\left(\tilde{A}_{\varepsilon}\right)$. Temos

$$
\tilde{a}_{\varepsilon}(u, v)=\left\langle\tilde{A}_{\varepsilon} u, v\right\rangle_{L^{2}\left(\Omega_{\varepsilon}\right)} \text {, qualquer que seja } v \in H^{1}\left(\Omega_{\varepsilon}\right) .
$$

O Lema 2.1.2 implica que

$$
a_{\varepsilon}\left(\Phi_{\varepsilon} u, \Phi_{\varepsilon} v\right)=\left\langle\Phi_{\varepsilon}\left(\tilde{\Lambda}_{\varepsilon} u\right), \Phi_{\varepsilon} v\right\rangle_{\left.L^{2}(\Omega)\right\rangle}, \text { qualquer que seja } v \in H^{1}\left(\Omega_{\varepsilon}\right) .
$$

Como $\Phi_{\varepsilon}: I^{1}\left(\Omega_{\Xi}\right) \longrightarrow H^{1}(\Omega)$ ó um isomorfismo, segue que

$$
a_{\varepsilon}\left(\Phi_{\varepsilon} u, w\right)=\left\langle\Phi_{\varepsilon}\left(\tilde{\Lambda}_{\varepsilon} u\right), w\right\rangle_{L^{2}(\Omega)} \text {, para qualquer que seja } w \in I^{1}(\Omega) .
$$

Ou seja, $\Phi_{\varepsilon} u \in D\left(\Lambda_{\varepsilon}\right)$ e $\Lambda_{\varepsilon}\left(\Phi_{\varepsilon} u\right)=\Phi_{\varepsilon}\left(\tilde{A}_{\varepsilon} u\right)$, mostrando a parte (2) do lema e que $\Phi_{\varepsilon}\left(D\left(\tilde{A}_{\varepsilon}\right)\right) \subset D\left(A_{\varepsilon}\right)$.

Por outro lado, seja $u \in D\left(A_{\varepsilon}\right)$. Então,

$$
a_{\varepsilon}(u, w)=\left\langle A_{\varepsilon} u, w\right\rangle_{L^{2}(\Omega)} \text { qualquer que seja } w \in H^{1}(\Omega) .
$$

Devemos mostrar que existe um $\tilde{u} \in D\left(\tilde{A}_{\varepsilon}\right)$ tal que $\Phi_{\varepsilon} \tilde{u}=u$. Como $\Phi_{\varepsilon}: H^{1}\left(\Omega_{\varepsilon}\right) \longrightarrow$ $I^{1}(\Omega)$ ć um isomorfismo, existe um $\tilde{u} \in H^{1}\left(\Omega_{\varepsilon}\right)$ tal que $\Phi_{\varepsilon} \tilde{u}=u$. Falta então mostrar que $\tilde{u} \in D\left(\tilde{A}_{\varepsilon}\right)$. Seja $v \in H^{1}\left(\Omega_{\varepsilon}\right)$. Então $w:=\Phi_{\varepsilon}(v) \in H^{1}(\Omega)$ e, portanto,

$$
a_{\tilde{\varepsilon}}\left(\Phi \tilde{u}, \Phi_{\varepsilon} v\right)=\left\langle A_{\varepsilon} \Phi_{\tilde{\varepsilon}} \tilde{u}, \Phi_{\varepsilon} v\right\rangle_{L^{2}(\Omega)}, \text { qualquer que seja } v \in H^{1}\left(\Omega_{\varepsilon}\right) .
$$

O Lema 2.1.2 implica que

$$
\tilde{a}_{\varepsilon}(\tilde{u}, v)=\left\langle\Phi_{\varepsilon}^{-1}\left(A_{\varepsilon}\left(\Phi_{\varepsilon} \tilde{u}\right)\right), v\right\rangle_{L^{2}\left(\Omega_{\varepsilon}\right)} \text { para qualquer que seja } v \in H^{1}(\Omega) .
$$

Logo, $\bar{u} \in D\left(\tilde{A}_{\varepsilon}\right)$ e $\tilde{A}_{\varepsilon} \tilde{u}=\Phi^{-1}\left(A_{\varepsilon}\left(\Phi_{\varepsilon} \tilde{u}\right)\right)$. Portanto $D\left(A_{\varepsilon}\right) \subset \Phi_{\varepsilon}\left(D\left(\tilde{A}_{\varepsilon}\right)\right)$.

Consideremos agora a seguinte hipótese sobre a função $f$ :

(HC1) $\int \in C^{1}(\mathbb{R})$ e existem constantes $C, \beta \in[0, \infty)$ tais que $\left|f^{\prime}(s)\right| \leq C\left(|s|^{\beta}+1\right)$ para todo $s \in \mathbb{R}$. Definamos $n:=M+N$. Se $n>2$, suponhamos também que $\beta \leq 2^{*} / 2-1$, onde $2^{*}=2 n /(n-2)>2$. 
Utilizando a teoria dos operadores de Nemitskii (ver por exemplo [1], [10] ou [27]) e o Teorema das Imersōes de Sobolev (ver, por exemplo, Teorema $0.4 \mathrm{em}$ [1]) vamos demonstrar o seguinte resultado:

Teorema 2.1.7 Suponhamos que $\int$ satisfaça $(\mathrm{HC1})$ e seja $\varepsilon>0$. Então o operador de Nemitskî $\tilde{f}: H^{1}\left(\Omega_{\varepsilon}\right) \longrightarrow L^{2}\left(\Omega_{\varepsilon}\right)$ está bem definido, é Lipschitziano em subconjuntos limitados de $H^{1}\left(\Omega_{\varepsilon}\right)$ e aplica subconjuntos limitados de $H^{1}\left(\Omega_{\varepsilon}\right)$ em subconjuntos limitados de $L^{2}\left(\Omega_{\varepsilon}\right)$.

Demonstração. Mostremos que se $u \in H^{1}\left(\Omega_{\bar{\varepsilon}}\right)$, então $\hat{f}(u) \in L^{2}\left(\Omega_{\varepsilon}\right)$. Como

$$
\left|f^{\prime}(s)\right| \leq C\left(|s|^{\beta}+1\right) \text { para todo } s \in \mathbb{R},
$$

temos que existem constantes positivas $C_{1}$ e $C_{2}$ tais que

$$
|f(s)| \leq C_{1}|s|^{\beta+1}+C_{2} \text { para todo } s \in \mathbb{R} .
$$

Elevando ao quadrado ambos os lados da desigualdade acima obtemos

$$
|f(s)|^{2} \leq C_{1}^{2}|s|^{2(\beta+1)}+2 C_{1} C_{2}|s|^{\beta+1}+C_{2}^{2} \text { para todo } s \in \mathbb{R} .
$$

A Desigualdade de Young implica que

$$
2 C_{1} C_{2}|s|^{\beta+1} \leq 1 / 2\left(\left(2 C_{1} C_{2}\right)^{2}+|s|^{2(\beta+1)}\right) .
$$

Logo, existem constantes $C_{3}, C_{4}>0$ tais que

$$
|f(s)|^{2} \leq C_{3}|s|^{2(\beta+1)}+C_{4} \text { para todo } s \in \mathbb{R} .
$$

Assim, se $u \in I^{1}\left(\Omega_{\varepsilon}\right)$, segue que

$$
\int_{\Omega_{\varepsilon}}|f(u(x, y))|^{2} \mathrm{~d} x \mathrm{~d} y<C_{3} \int_{\Omega .}|u(x, y)|^{2(\beta+1)} \mathrm{d} x \mathrm{~d} y+C_{5}=C_{3}|u|_{L^{2}(\beta+1)}^{2(\beta+1)}+C_{5},
$$

onde $C_{5}:=\mu\left(\Omega_{\bar{\varepsilon}}\right) C_{4}$.

As hipóteses sobre $\beta$ e o Teorema das Imersões de Sobolev implicam que $H^{1}\left(\Omega_{\varepsilon}\right)$ está continuamente imerso em $L^{2(\beta+1)}\left(\Omega_{\varepsilon}\right)$. Assim, existe uma constante $C_{6}>0$ tal que

$$
\int_{\Omega_{\varepsilon}}|f(u(x, y))|^{2} \mathrm{~d} x \mathrm{~d} y \leq C_{6}|u|_{I I^{1}\left(\Omega_{\mathrm{s}}\right)}^{2(\beta+1)}+C_{5}
$$

Portanto, $\tilde{f}: H^{1}\left(\Omega_{\varepsilon}\right) \longrightarrow L^{2}\left(\Omega_{\varepsilon}\right)$ está bem definido. Além disso,

$$
|\tilde{f}(u)|_{L^{2}\left(\Omega_{s}\right)} \leq\left(C_{6}|u|_{H^{1}\left(\Omega_{\varepsilon}\right)}^{2(\beta+1)}+C_{5}\right)^{1 / 2}
$$

ou seja, $\tilde{f}$ aplica subconjuntos limitados de $H^{1}\left(\Omega_{\varepsilon}\right)$ em subconjuntos limitados de $I^{2}\left(\Omega_{\varepsilon}\right)$. 
Mostremos agora que $\tilde{f}$ é uma função Lipschitziana em subconjuntos limitados de $H^{1}\left(\Omega_{\varepsilon}\right)$, isto é, para quaisquer que sejam $u, v \in H^{1}\left(\Omega_{\varepsilon}\right)$ tais que $|v|_{\left[T_{1}\left(\Omega_{-}\right)\right.} \leq R$ e $|u|_{H I^{1}\left(\Omega_{\varepsilon}\right)} \leq$ $R$, com $R<\infty$, então existe uma constante $L>0$ tal que

$$
|\hat{f}(u)-\tilde{f}(v)|_{L^{2}\left(\Omega_{\varepsilon}\right)} \leq L|u-v|_{H^{1}\left(\Omega_{\varepsilon}\right)} .
$$

Para isso notemos inicialmente que, quaisquer que sejam $u, v \in H^{1}\left(\Omega_{\varepsilon}\right)$ e $(x, y) \in \Omega_{\varepsilon}$, temos

$$
f(u(x, y))-f(v(x, y))=(u(x, y)-v(x, y)) \int_{0}^{\mathrm{i}} f^{\prime}(t u(x, y)+(1-t) v(x, y)) \mathrm{d} t .
$$

Logo, segue de (2.2) que

$$
\begin{aligned}
|f(u(x, y))-f(v(x, y))| & \leq|u(x, y)-v(x, y)| \int_{0}^{1}\left|f^{\prime}(t u(x, y)+(1-t) v(x, y))\right| \mathrm{d} t \\
& \leq|u(x, y)-v(x, y)| \int_{0}^{1} C\left(1+(|u(x, y)|+|v(x, y)|)^{\beta}\right) \mathrm{d} t \\
& \leq C|u(x, y)-v(x, y)|\left(1+2^{\beta} \max \left\{|u(x, y)|^{\beta},\left.j v(x, y)\right|^{\beta}\right\}\right) \\
& \leq C|u(x, y)-v(x, y)|\left(1+2^{\beta}|u(x, y)|^{\beta}+2^{\beta}|v(x, y)|^{\beta}\right) \\
& \leq C_{7}|u(x, y)-v(x, y)|\left(1+|u(x, y)|^{\beta}+|v(x, y)|^{\beta}\right) \\
& \leq C_{7}|u(x, y)-v(x, y)|\left(3 \max \left\{1,|u(x, y)|^{\beta},|v(x, y)|^{\beta}\right\}\right),
\end{aligned}
$$

onde $C_{T}^{\prime}=\max \left\{C^{\prime}, 2^{\beta} C^{\prime}\right\}$.

Elevando ao quadrado ambos os lados da desigualdade acima encontramos:

$$
\begin{aligned}
|f(u(x, y))-f(v(x, y))|^{2} & \leq C_{7}^{2}|u(x, y)-v(x, y)|^{2}\left(3^{2} \max \left\{1,|u(x, y)|^{2 \beta}:|v(x, y)|^{2 \beta}\right\}\right) \\
& \leq C_{8}|u(x, y)-v(x, y)|^{2}\left(1+|u(x, y)|^{2 \beta}+|v(x, y)|^{2,3}\right),
\end{aligned}
$$

onde $C_{8}=\left(3 C_{7}\right)^{2}$. Integrando em $\Omega_{\varepsilon}$

$$
\begin{aligned}
\int_{\Omega_{s}}|f(u(x, y))-f(v(x, y))|^{2} \mathrm{~d} x \mathrm{~d} y \\
\quad \leq C_{8} \int_{\Omega_{\varepsilon}}|u(x, y)-v(x, y)|^{2}\left(1+|u(x, y)|^{2 \beta 3}+v(x, y)^{2 \beta 3}\right) \mathrm{d} x \mathrm{~d} y \\
\quad=C_{8} \int_{\Omega_{\varepsilon}}^{n}|u(x, y)-v(x, y)|^{2} \mathrm{~d} x \mathrm{~d} y+C_{8} \int_{\Omega_{\varepsilon}}|u(x, y)|^{2 \beta}|u(x, y)+v(x, y)|^{2} \mathrm{~d} x \mathrm{~d} y \\
\quad+C_{8} \int_{\Omega_{\varepsilon}}|v(x, y)|^{2 \beta}|u(x, y)+v(x, y)|^{2} \mathrm{~d} x \mathrm{~d} y .
\end{aligned}
$$

Definamos agora $p:=2^{*} /(2 \beta)>1$ e $q:=p /(p-1)=2^{*} /\left(2^{*}-2 \beta\right)$. A Desigualdade de Hölder implica que

$$
\begin{aligned}
\int_{\Omega_{\varepsilon}} \mid f(u(x, y))- & \left.\int(v(x, y))\right|^{2} \mathrm{~d} x \mathrm{~d} y \\
& \leq C_{8}\left(|u-v|_{L^{2}\left(\Omega_{\varepsilon}\right)}^{2}+|u|_{L^{2^{-}}\left(\Omega_{\varepsilon}\right)}^{2,3}|u-v|_{L^{2 q}\left(\Omega_{\xi}\right)}^{2}+|v|_{L^{2^{*}}\left(\Omega_{\varepsilon}\right)}^{2,3}|u-v|_{L^{2 q}\left(\Omega_{\varepsilon}\right)}^{2}\right) .
\end{aligned}
$$


Observemos que $2 \leq 2 q \leq 2^{*}$. Logo, $H^{1}\left(\Omega_{\varepsilon}\right)$ está continuamente imerso em $L^{2^{*}}\left(\Omega_{\varepsilon}\right)$ e em $L^{2 q}\left(\Omega_{\bar{\varepsilon}}\right)$. Portanto, existe uma constante positiva $C_{9}$ tal que

$$
\int_{\Sigma_{\Omega_{E}}}|f(u(x, y))-f(v(x, y))|^{2} \mathrm{~d} x \leq C_{9}\left(1+|u|_{H^{1}\left(\Omega_{\epsilon}\right)}^{2 \beta}+|v|_{H^{1}\left(\Omega_{\epsilon}\right)}^{2 \beta}\right)|u-v|_{H^{1}\left(\Omega_{\varepsilon}\right)}^{2} .
$$

Se $u, v \in H^{1}\left(\Omega_{\varepsilon}\right)$ são tais que $|u|_{H^{1}\left(\Omega_{\varepsilon}\right)},|\eta|_{H^{1}\left(\Omega_{\varepsilon}\right)} \leq R$, segue que

$$
|\tilde{f}(u)-\tilde{f}(v)|_{L^{2}\left(\Omega_{\varepsilon}\right)} \leq C_{9}^{1 / 2}\left(1+2 R^{2 \beta}\right)^{1 / 2}|u-v|_{H^{1}\left(\Omega_{\epsilon}\right)} .
$$

Portanto, $\tilde{f}$ é Lipschitziano em subconjuntos limitados de $I^{1}\left(\Omega_{\varepsilon}\right)$.

No restante dessa seção vamos assumir que $f$ satisfaça a hipótese (HC1).

Dado $\varepsilon>0$, definimos a função $\hat{f}: H^{1}(\Omega) \longrightarrow L^{2}(\Omega)$ por $\hat{f}:=\Phi_{\varepsilon} \circ \tilde{f} \circ \Phi_{=}^{-1}$. A boa definição de $\hat{f}$ segue do seguinte diagrama:

$$
H^{1}(\Omega) \stackrel{\Phi_{\varepsilon}^{-1}}{\longrightarrow} H^{1}\left(\Omega_{\varepsilon}\right) \stackrel{f}{\longrightarrow} L^{2}\left(\Omega_{\varepsilon}\right) \stackrel{\Phi_{c}}{\longrightarrow} L^{2}(\Omega) .
$$

Notemos que $\hat{f}$ não depende do parâmetro $\varepsilon$. De fato, para quaisquer que sejam $u \in H^{1}(\Omega)$ e $(x, y) \in \Omega$,

$$
\Phi_{\varepsilon} \circ \tilde{f} \circ \Phi_{\varepsilon}^{-1}(u)(x, y)=f \circ \Phi_{\varepsilon}^{-1} \circ u(x, \varepsilon y)=f \circ u(x, y)=f(u(x, y)) .
$$

A igualdade acima mostra que $\hat{\jmath}$ é o operador de Nenitskiĭ de $H^{1}(\Omega) \mathrm{cm} L^{2}(\Omega)$ associado a $f$. Além disso, a hipótese (HC1) implica que $H^{1}(\Omega)$ está continuamente

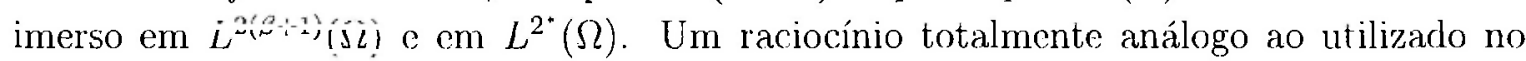
Teorema 2.1.7 nos permite concluir o seguinte resultado:

Teorema 2.1.8 A aplicação $\hat{f}: I^{1}(\Omega) \longrightarrow L^{2}(\Omega)$ e Lipschitziana em subconjuntos limitados de $H^{1}(\Omega)$ e aplica subconjuntos limitados de $H^{1}(\Omega)$ em subconjuntos limitados de $L^{2}(\Omega)$.

Os Teoremas 2.1.5, 2.1.7 e 2.1.8 implicam que as seguintes equações de evolução estão bern clefinidas:

$$
\begin{aligned}
& \dot{u}+\tilde{A}_{\varepsilon} u=\tilde{f}(u) \\
& \dot{u}+A_{\varepsilon} u=\hat{f}(u)
\end{aligned}
$$

Notemos que, para cada $\varepsilon>0,\left(\tilde{R D_{\varepsilon}}\right)$ é a formulação abstrata de $\left(\tilde{E}_{z}\right)$. Além disso, $\left(R D_{\varepsilon}\right)$ é a formulação abstrata de

$$
\begin{array}{ll}
u_{t}=\Delta_{x} u+\frac{1}{\varepsilon^{2}} \Delta_{y} u+f(u), & t>0,(x, y) \in \Omega \\
\partial_{\nu_{r}} u+\frac{1}{\varepsilon^{2}} \partial_{\nu_{y}} u=0, & t>0,(x, y) \in \partial \Omega .
\end{array}
$$

As Proposições 1.1.7 e 1.1.8 implicam no seguinte fato: 
Proposição 2.1.9 Para cada $\varepsilon>0$, o operador linear $\bar{A}_{\varepsilon}$ (respectivamente, $A_{\varepsilon}$ ) é setorial em $X-L^{2}\left(\Omega_{\varepsilon}\right)$ (respectivamente, $X=L^{2}(\Omega)$ ) e o espaço de potências fracionárias correspondente a $\alpha=1 / 2$ satisfaz $X^{\alpha}=H^{1}\left(\Omega_{\varepsilon}\right)$ (respectivamente, $X^{\alpha}=H^{1}(\Omega)$ ).

A Proposição 2.1.9 e os Teoremas 2.1 .7 e 2.1.8, juntamente com uma aplicação do Exemplo 1.2.2 implicam que, para cada $\varepsilon>0$, a equação $\left(R D_{\varepsilon}\right)$ (respectivamente, $\left(R D_{\Xi}\right)$ ), gera um scmifluxo local $\tilde{\pi}_{\varepsilon}$ em $H^{1}\left(\Omega_{\varepsilon}\right)$ (respectivamente, $\pi_{\varepsilon}$ em $H^{1}(\Omega)$ ). A relação entre tais semifluxos locais é expressa no próximo resultado.

Proposição 2.1.10 Para cada $\varepsilon>0$, a aplicação $\Phi_{s}$ é uma conjugação entre os semifluxos locais $\tilde{\pi}_{\varepsilon}$ e $\pi_{\varepsilon}$, isto é, dados $u \in H^{1}(\Omega)$ e $t>0$, então u$\pi_{\varepsilon} t$ está definido se, $e$ somente se $\left(\Phi_{\varepsilon}^{-1} u\right) \tilde{\pi}_{\varepsilon} t$ cstá definido e neste caso $u \pi_{\varepsilon} t=\Phi_{\varepsilon}\left(\left(\Phi_{\varepsilon^{*}}^{-1} u\right) \tilde{\pi}_{s} t\right)$.

Demonstração. Fixemos $\varepsilon>0$ e seja $\tilde{u}_{0} \in H^{1}\left(\Omega_{\varepsilon}\right)$. Suponhamos que $\tilde{u}_{0} \tilde{\pi}_{\varepsilon} t$ esteja definiclo se, e somente se, $t \in\left[0, \omega_{i_{0}}\right)$.

Definimos $u_{0}:=\Phi_{\varepsilon}\left(\tilde{u}_{0}\right)$ e suponhamos que $u_{0} \pi_{\varepsilon} l$ csteja definiclo se, c somente se, $t \in\left[0, \omega_{u_{0}}\right)$. Consideraremos duas situções.

(a) Sc $t \in\left[0, \omega_{\bar{u}_{0}}\right)$, mostremos que $\ell \in\left[0, \omega_{u_{0}}\right)$ e que

$$
u_{0} \pi_{\varepsilon} t=\Phi_{\varepsilon}\left(\bar{u}_{0} \tilde{\pi}_{\varepsilon} t\right)=\Phi_{\varepsilon}\left(\left(\Phi_{\varepsilon}^{-1} u_{0}\right) \tilde{\pi}_{\underline{\varepsilon}} t\right)
$$

Como $t \in\left[0, \omega_{i_{0}}\right)$ segue que $\tilde{u}_{0} \tilde{\pi}_{\varepsilon} t$ está definido e satisfaz a equação de evolução

$$
\dot{u}+\tilde{A}_{\varepsilon} u=\tilde{f}(u)
$$

isto ć,

$$
\frac{\mathrm{d}}{\mathrm{d} t}\left(\tilde{u}_{0} \tilde{\pi}_{\varepsilon} t\right)+\tilde{A}_{\varepsilon}\left(\tilde{u}_{0} \tilde{\pi}_{\varepsilon} t\right)=\tilde{f}\left(\tilde{u}_{0} \tilde{\pi}_{\varepsilon} t\right)
$$

Calculemos $\frac{\mathrm{d}}{\mathrm{d} t}\left(\Phi_{\varepsilon}\left(\tilde{u}_{0} \tilde{\pi}_{\varepsilon} t\right)\right)$. É claro que $\frac{\mathrm{d}}{\mathrm{d} t}\left(\omega_{\varepsilon}\left(\tilde{u}_{0} \ddot{\pi}_{\varepsilon} t\right)\right)=\Phi_{\varepsilon}\left(\frac{\mathrm{d}}{\mathrm{d} t}\left(\tilde{u}_{0} \tilde{\pi}_{\varepsilon} t\right)\right)$. Utilizando (2.5) e o fato de $\Phi_{\varepsilon}$ ser linear temos que

$$
\frac{\mathrm{d}}{\mathrm{d} t}\left(\Phi_{\varepsilon}\left(\tilde{u}_{0} \bar{\pi}_{\varepsilon} t\right)\right)=\Phi_{\varepsilon}\left(-\tilde{A}_{\varepsilon}\left(\tilde{u}_{0} \bar{\pi}_{\varepsilon} t\right)\right)+\Phi_{\equiv}\left(\tilde{f}\left(\tilde{u}_{0} \tilde{\pi}_{\varepsilon} t\right)\right)
$$

Como $\tilde{u}_{0} \tilde{\pi}_{=} l \in D\left(\tilde{\Lambda}_{\varepsilon}\right)$, o Lema 2.1.6 implica que

$$
\Phi_{\varepsilon}\left(\tilde{u}_{0} \tilde{\pi}_{\varepsilon} t\right) \in D\left(A_{\varepsilon}\right) \text { e } A_{\Sigma}\left(\Phi_{\varepsilon}\left(\tilde{u}_{0} \tilde{\pi}_{\varepsilon} t\right)\right)=\Phi_{\hat{\varepsilon}}\left(\tilde{A}_{\varepsilon}\left(\tilde{u}_{0} \tilde{\pi}_{\varepsilon} t\right)\right) .
$$

Além disso, como $\hat{f}=\Phi_{\varepsilon} \circ \tilde{f} \circ \Phi_{\varepsilon}^{-1}$, segue que $\hat{f} \circ \Phi_{\varepsilon}=\bar{\Psi}_{\varepsilon} \circ \tilde{f}$. Portanto,

$$
\Phi_{\varepsilon}\left(\tilde{f}\left(\tilde{u}_{0} \tilde{\pi}_{\varepsilon} t\right)\right)=\hat{f}\left(\Phi_{\varepsilon}\left(\tilde{u}_{0} \tilde{\pi}_{\varepsilon} t\right)\right) .
$$

Substituindo esses fatos em (2.6) segue que

$$
\frac{\mathrm{d}}{\mathrm{d} t}\left(\Phi_{\tilde{\varepsilon}}\left(\tilde{u}_{0} \tilde{\pi}_{\varepsilon} t\right)\right)=-A_{\varepsilon}\left(\Phi_{\varepsilon}\left(\tilde{u}_{0} \tilde{\pi}_{\varepsilon} t\right)\right)+\hat{f}\left(\Phi_{\varepsilon}\left(\tilde{u}_{0} \tilde{\pi}_{\varepsilon} t\right)\right),
$$


isto é, $\Phi_{\Xi}\left(\tilde{u}_{1}, \tilde{\pi}_{\tilde{\varepsilon}} t\right)$ satisfaz a equação de evolução $\dot{u}+A_{\varepsilon} u=\hat{f}(u)$. Como $\Phi_{\varepsilon}\left(\tilde{u}_{0} \tilde{\pi}_{\varepsilon} 0\right)=u_{0}$, segue que $t \in\left[0, \omega_{u_{0}}\right)$ e $u_{i}, \pi_{s} t=\Phi_{\varepsilon}\left(\ddot{u}_{0} \tilde{\pi}_{s} t\right)$

(b) Suponhamos agora que $\iota \in\left[0, \omega_{u_{0}}\right)$. Do mesmo modo que em (a), demosntramos que $t \in\left[0, \omega_{u_{0}}\right)$ e $\tilde{u}_{0} \tilde{\pi}_{\varepsilon} t=\Phi_{\varepsilon}^{-1}\left(u_{0} \pi_{s} t\right)$.

Isso conclui a demonstração.

A Proposição 2.1.10 nos motiva a estudar, para cada $\varepsilon>0$, a equação de evolução

$$
\dot{u}+A_{\varepsilon} u=\hat{f}(u),
$$

uma vez que as propriedades de $\left(R D_{\varepsilon}\right)$ são transferidas para $\left(\tilde{R D_{\varepsilon}}\right)$ via a conjugação $\Phi_{\varepsilon}$.

\subsection{Os espaços $t t_{\mathrm{s}}^{1}(O)$ e $L_{s}^{2}(O)$}

Queremos apresentar o problema limite para a família de equaçöes $\left(R D_{\varepsilon}\right)$ com $\varepsilon \in$ $(0,1]$. Iniciamos definindo os espaços nos quais o problema limite estará definido. Tal definição será apresentadia nesta seção. Antes porém neecessitamos de alguns resultados auxiliares.

Usaremos a notação $\nabla_{y} u$ significando o vetor $\left(\partial_{y_{1}} u \ldots, \partial_{y, \mathrm{~N}} u\right)$, onde $\partial$ é a derivada parcial no sentido das distribuições. A medida de Lebesgue em $\mathbb{F}^{\prime \prime}$ será denotada por $\mu_{k}$, para qualquer $k \in \mathbb{N}$. Começamos com um resultado da Teoria de Distribuições que utilizaremos.

Lema 2.2 .1 (cf. [2]) Sejam $O_{1}$ e $O_{2}$ subconjuntos abertos de $\mathbb{R}^{M}$ e de $\mathbb{R}^{N}$ respectivamente. Considere o conjunto $\mathcal{D}\left(O_{1}\right) \otimes \mathcal{D}\left(O_{2}\right)$ formado pelas funçôes $u: O_{1} \times O_{2} \longrightarrow \mathbb{R}$ dadas como somas finitas

$$
u(x, y)=\sum_{i=1}^{k} \phi_{i}(x) \psi_{i}(y)
$$

onde $\phi_{i} \in \mathcal{D}\left(O_{1}\right), \psi_{\imath} \in \mathcal{D}\left(O_{2}\right), i=i_{1} \ldots \dot{\kappa}$; $\quad$ e $k \in \mathbb{N}$. Então $\mathcal{D}\left(O_{1}\right) \otimes \mathcal{D}\left(O_{2}\right)$ é um subespaço denso de $\mathcal{D}\left(\mathrm{O}_{1} \times \mathrm{O}_{2}\right)$.

Nosso primeiro resultado auxiliar é dado a seguir.

Lema 2.2.2 Seja $O \subset \mathbb{R}^{M+N}$ um conjunto aberto da forma $O-U \times V$, onde $U \subset \mathbb{R}^{M}$ e $V \subset \mathbb{R}^{N}$ são retângulos abertos limitados. Seja $u \in L_{1 \mathrm{loc}}^{1}(O)$ com $\nabla_{y} u=0$. As seguintes propriedades estão satisfeitas:

(1) existe um conjunto de medida nula $S \subset \mathbb{R}^{M+N}$ e uma função $v \in L_{\text {loc }}^{1}(U)$ tal que $u(x, y)=v(x)$ para todo $(x, y) \in O \backslash S$;

(2) se $\partial_{x_{i}} u \in L_{\text {loc }}^{1}(O)$ para algum $i=1, \ldots, \hat{h i}$, entâo $\partial_{x_{i}} v \in L_{\text {loc }}^{1}(U)$ e existe um conjunto de medida nula $S^{\prime} \subset \mathbb{R}^{M+N}$ tal que $u(x, y)=v(r)$ e $\partial_{x_{i}} u(x, y)=\partial_{x_{i}} v(x)$ para $(x, y) \in O \backslash S^{\prime}$; 
(3) se $u \in H^{1}(O)$, então $v \in H^{1}(U)$, onde $v$ é a função obtida em (1);

(4) se $u \in H^{1}(O)$ e $M=1$, então existe um conjunto de medida nula $S^{\prime \prime} \subset \mathbb{R}^{1+*}$ e uma função absolutamente contínua $\hat{v} \in H^{1}(U)$ tal que $u(x, y)=\tilde{v}(x)$ i $\partial_{x} u(x, y)=$ $\partial_{x} \bar{v}(x)$ para $(x, y) \in O \backslash S^{\prime \prime}$. Em particular, $u(x, y)=\tilde{u}(x, y)$ em $O \backslash S^{\prime \prime}$, onde $\tilde{u}: U \times V \rightarrow \mathbb{R}$ é a função contínua dada por $\tilde{u}(x, y):=\tilde{v}(x)$ para $(x, y) \in U \times V$.

Demonstração. Como $U \subset \mathbb{R}^{M}$ e $V \subset \mathbb{R}^{N}$ são retângulos limitados, podemos representá-los por $U=\left(a_{1}, b_{1}\right) \times \cdots \times\left(a_{M}, b_{M}\right)$ e $V=\left(c_{1}, d_{1}\right) \times \cdots \times\left(c_{N}, \dot{a}_{N}\right)$, onde $a_{i}, b_{i}, c_{j}, d_{j} \in \mathbb{R}, i=1, \ldots, M$ e $j=1, \ldots, N$.

Como $\nabla_{y} u=0$, segue que $\partial_{y_{N}} u=0$. Defina $\widetilde{V}:=\left(c_{1}, d_{1}\right) \times \ldots \times\left(c_{N-1}, d_{N-1}\right) \subset \mathbb{R}^{N-1}$ e consideremos $\phi \in \mathcal{D}(U \times \widetilde{V})$ e $\psi \in \mathcal{D}\left(\left(c_{N}, d_{N}\right)\right)$. Logo, $\phi \dot{v} \in \mathcal{D}(U \times V)$ e

$$
\int_{I_{O}} u(x, y) \phi\left(x, y_{1}, \ldots, y_{N-1}\right) \psi^{\prime}\left(y_{N}\right) \mathrm{d} x \mathrm{~d} y=0 .
$$

Seja $\alpha \in \mathcal{D}\left(\left(c_{N}, d_{N}\right)\right)$ tal que

$$
\int_{c_{c_{N}}}^{d_{N}} \alpha\left(y_{N}\right) \mathrm{d} y_{N}=1
$$

Então, para toda função $\beta \in \mathcal{D}\left(\left(c_{N}, d_{N}\right)\right)$, definimos

$$
\theta(s):=\int_{c_{N}}^{s} \beta(t) \mathrm{d} t-\int_{c_{N}}^{d_{N}} \beta(s) \mathrm{d} s{f_{c_{N}}^{r s}}_{c^{\prime}} \alpha(t) \mathrm{d} t \text { para } s \in\left(c_{N}, d_{N}\right) .
$$

Segue que $\theta \in \mathcal{D}\left(\left(c_{N}, d_{N}\right)\right)$. Portanto, para toda função $\beta \in \mathcal{D}\left(\left(c_{N}, d_{N}\right)\right)$, temos que

$$
\beta-\left(\int_{c_{N}}^{d_{N}} \beta(s) \mathrm{d} s\right) \alpha
$$

é a derivada de uma função em $\mathcal{D}\left(\left(c_{N}, d_{N}\right)\right)$. Assim, tomando $\beta=\psi$ temos que

$$
\int_{0} u(x, y) \phi\left(x, y_{1}, \ldots, y_{N-1}\right)\left(\psi\left(y_{N}\right)-\left(\int_{r^{\prime}}^{a_{N}} \psi(s) \mathrm{d} s\right) \alpha\left(y_{N}\right)\right) \mathrm{d} x \mathrm{~d} y=0,
$$

ou seja,

$$
\begin{aligned}
\int_{O} u(x, y) \phi\left(x, y_{1}, \ldots, y_{N-1}\right) \psi\left(y_{N}\right) \mathrm{d} x \mathrm{~d} y \\
=\left(\int_{c_{*}}^{i_{N}} \psi(s) \mathrm{d} s\right)\left(\int_{O} u(x, y) \phi\left(x, y_{1}, \ldots, y_{N-1}\right) \alpha\left(y_{N}\right) \mathrm{d} x \mathrm{~d} y\right) .
\end{aligned}
$$

Agora definimos

$$
v\left(x, y_{1}, \ldots, y_{N-1}\right):=\int_{c_{N}}^{d_{N}} u\left(x, y_{1}, \ldots, y_{N}\right) \alpha\left(y_{N}\right) \mathrm{d} y_{N} .
$$


Segue do Teorema de Fubini que $v \in L_{\text {loc }}^{1}(U \times \widetilde{V})$. Além disso, podemos considerar $v$ como uma função em $O$ e neste caso $v \in L_{\text {loc }}^{1}(O)$. Com isso,

$$
\begin{aligned}
\int_{O} u(x, y) \phi( & \left.x, u_{1}, \ldots, y_{N-1}\right) \psi\left(y_{N}\right) \mathrm{d} x \mathrm{~d} y \\
& =\left(\int_{c_{N}}^{d_{N}} \psi(s) \mathrm{d} s\right)\left(\int_{J_{U \times} \tilde{V}} v\left(x, y_{1}, \ldots, y_{N^{\prime}-1}\right) \phi\left(y_{1}, \ldots, y_{N-1}\right) \mathrm{d} x \mathrm{~d} y_{1} \ldots \mathrm{d} y_{N-1}\right) \\
& \left.=\int_{J_{O}} v\left(x, y_{1}, \ldots, y_{N-1}\right) \phi\left(y_{1}, \ldots, y_{N-1}\right) \psi_{(}\right) \mathrm{d} x \mathrm{~d} y .
\end{aligned}
$$

Portanto, para quaisquer que sejam $\phi \in \mathcal{D}(U \times \widetilde{V})$ e $\omega \in \mathcal{D}\left(\left(c_{N}, d_{N}\right)\right)$, temos que

$$
\int_{I_{O}}\left(u(x, y)-v\left(x, y_{1}, \ldots, y_{N-1}\right)\right) \phi\left(y_{1}, \ldots, y_{N-1}\right) \psi\left(y_{N}\right) \mathrm{d} x \mathrm{~d} y=0 .
$$

Segue do Lema 2.2.1 que o conjunto gerado por todas as funções da forma $\phi \psi$, onde $\phi \in \mathcal{D}(U \times \widetilde{V})$ e $\psi \in \mathcal{D}\left(\left(c_{N}, d_{N}\right)\right)$ é denso em $\mathcal{D}(O)$. Logo,

$$
\int_{O}\left(u(x, y)-v\left(x, y_{1}, \ldots, y_{N-1}\right)\right) \gamma(x, y) \mathrm{d} x \mathrm{~d} y=0, \text { qualquer que seja } \gamma \in \mathcal{D}(O) .
$$

Assim, $u-v=0$ quase sempre em $O$, isto é, uxiste um conjunto do medida nula $S_{N} \subset \mathbb{R}^{M+N}$ tal que

$$
u(x, y)=v\left(x, y_{1}, \ldots, y_{N-1}\right) \text { para todo }(x, y) \in O \backslash S .
$$

Afirmamos que $\partial_{y_{j}} v=0$, para $j=1, \ldots, N-1$. De fato, seja $\phi \in \mathcal{D}(U \times \widetilde{V})$. Para cada $j=1, \ldots, N-1$,

$$
\begin{aligned}
\int_{U \times \tilde{V}} v\left(x, y_{1}, \ldots, y_{N-1}\right) \partial_{y_{j}} \phi\left(x, y_{1}, \ldots, y_{N-1}\right) \mathrm{d} x \mathrm{~d} y_{1} \ldots \mathrm{d} y_{N-1} \\
\quad=\int_{U_{U \times V}}\left(\int_{c_{N}}^{d_{N}} u\left(x, y_{1}, \ldots, y_{N}\right) \alpha\left(y_{N}\right) \mathrm{d} y_{N}\right) \partial_{J_{j}} o\left(x, y_{1} \ldots, y_{N-1}\right) d x \mathrm{~d} y_{1} \ldots \mathrm{d} y_{N-1} \\
\quad=\int_{i_{O}} u(x, y) \partial_{y_{j}}\left(\phi\left(x, y_{1}, \ldots, y_{N-1}\right) \alpha\left(y_{N}\right)\right) \mathrm{d} x \mathrm{~d} y=0
\end{aligned}
$$

e nossa afirmativa está demonstrada.

Agora, com um processo de indução $\mathrm{cm} N$, obtemos um conjunto de medida nula $S \subset \mathbb{R}^{M+N}$ e $v \in L_{\text {loc }}^{1}(U) \operatorname{com} u(x, y)=v(x)$ para todo $(x, y) \in O \backslash S$. A demonstração de (1) está concluíla.

Mostremos (2). Assumimos entâo que $\partial_{x_{i}} u \in L_{\text {loc }}^{1}(O)$ para algum $i=1, \ldots, M$. Observemos que $\nabla \partial_{x_{i}} u=\partial_{x_{i}} \nabla u=0$ no sentido das distribuições. A primeira parte deste lema aplicada à função $\partial_{x_{i}} u$ implica que existe um conjunto de medida nula $S^{\prime} \subset \mathbb{R}^{M+N}$ 
e $w \in L_{\text {loc }}^{1}(U) \operatorname{com} \partial_{x_{i}} u(x, y)=w(x)$, para todo $(x, y) \in \Omega \backslash S^{\prime}$. Assim, para quaisquer que sejam $\phi \in \mathcal{D}(U)$ e $\psi \in \mathcal{D}(V)$ temos

$$
\begin{aligned}
\int_{U} \partial_{x_{i}} v(x) \phi(x) \mathrm{d} x \int_{V} \psi(y) \mathrm{d} y & =-\int_{U} v(x) \partial_{x_{i}} \phi(x) d x \int_{V} \psi(y) \mathrm{d} y \\
& =-\int_{U \times V} v(x) \psi(y) \partial_{x_{i}} \phi(x) \mathrm{d} x \mathrm{~d} y \\
& =-\int_{U \times V} u(x, y) \partial_{x_{i}}(\phi(x) \psi(y)) \mathrm{d} x \mathrm{~d} y \\
& =\int_{U \times V} \partial_{x_{i}} u(x, y) \phi(x) \psi(y) \mathrm{d} x \mathrm{~d} y \\
& =\int_{U \times V} u(x) \phi(x) \psi(y) \mathrm{d} x \mathrm{~d} y \\
& =\int_{U} w(x) \phi(x) \mathrm{d} x \int_{V} \psi(y) \mathrm{d} y
\end{aligned}
$$

Escolhendo $\psi \in \mathcal{D}(V)$ de forma que

$$
\int_{V} \psi(y) \mathrm{d} y=1
$$

vemos que $\partial_{x_{i}} v=w$, demonstrando (2).

Agora, se $u \in H^{1}(O)$, então $u \in L^{2}(O)$ e $\partial_{x_{i}} u \in L^{2}(O)$ para $i=1, \ldots, M$. Então, o mesmo raciocínio acima nos diz que $v: \in H^{1}(U)$ c isto prova (3)

Suponha que as hipóteses de (4) estejam verificadas. Então, segue do Teorema VIII.2 em [3] que existe um conjunto de medida nula $Z \subset U$ e uma função absolutamente contínua $\tilde{v} \mathrm{em} U$ tal que $v(x)=\tilde{v}(x) \mathrm{cm} U \backslash Z$. Como $Z \times V$ é de medida nula em $U \times V$, segue do que já foi demonstrado que $u(x, y)=\tilde{v}(x)$ em $O \backslash S^{\prime \prime}$, para algum conjunto de medida nula $S^{\prime \prime} \subset \mathbb{R}^{M+N}$

$\mathrm{Na}$ verdade, a condição (4) do Lema 2.2.2 é válida quando $O$ é um aberto qualquer de $\mathbb{R}^{M+N}$ com $M=1$. A demonstração desse fato é o objetivo do próximo resultado

Proposição 2.2.3 Seja $O \subset \mathbb{R}^{M+N}$ um conjunto aberto arbitrário com $M=1$. Suponha que $u \in H^{1}(O)$ satisfaça $\nabla_{y} u=0$. Então u possui um representante contínuo, isto é, existe uma função contínua $\tilde{u}: O \longrightarrow \mathbb{R}$ e um conjunto de medida nula $S \subset \mathbb{R}^{M+N}$ tal que $u(x, y)=\tilde{u}(x, y)$ para todo $(x, y) \in O \backslash S$.

Demonstração. Seja $\left(O_{n}\right)_{n \in \mathbb{N}}$ uma cobertura de $O$ onde $O_{n}, n \in \mathbb{N}$, é produto cartesiano de retângulos abcrtos, isto é, para cada $n \in \mathbb{N}, O_{n}=U_{n} \times V_{n}$, com $U_{n} \subset \mathbb{R}^{M} \mathrm{e} V_{n} \subset \mathbb{R}^{. V}$ retângulos abertos. O Lema 2.2.2 (4) implica que, para cada $n \in \mathbb{N}$, existe um conjunto de medida nula $S_{n} \subset \mathbb{R}^{I+N}$ e uma função contínua $u_{n}: O_{n} \rightarrow \mathbb{R}$ tal que $u(x, y)=u_{n}(x, y)$ sempre que $(x, y) \in O_{n} \backslash S_{n}$. Delina o conjunto $S:=\bigcup_{n=1}^{\infty} S_{n}$. Segue que $S$ possui medida 
nula em $\mathbb{R}^{M+N}$ e para quaisquer $m, n \in \mathbb{N}$, temos que $u_{m}(x, y)-u(x, y)-u_{n}(x, y)$, para $(x: y) \in O_{m} \cap O_{n} \backslash S$.

Afirmamos que $O_{m} \cap O_{n} \backslash S$ ó denso $\mathrm{cm} O_{m} \cap O_{n}$. Caso contrário, cxistiria um $\left(x_{0}, y_{0}\right) \in O_{m} \cap O_{n}$ e uma vizinhança aberta $W$ desse ponto tal que $W \cap \overline{\left(O_{m} \cap O_{n} \backslash S\right)}=\emptyset$. Logo $W \subset S$, o que contradiz o fato de $S$ possuir medida nula. Nossa afirmação está demonstrada.

A continuidade de $u_{m}$ e $u_{n}$ e a afirmação acima implicam que $u_{m}(x, y)=u_{n}(x, y)$ sempre que $(x, y) \in O_{m} \cap O_{n}$. Com isso, existe uma única função $\tilde{u}: O \longrightarrow \mathbb{R}$ tal que $\left.\tilde{u}\right|_{O_{n}}=u_{n}$ para todo $n \in \mathbb{N}$. Segue que $\tilde{u}$ é uma função contínua e $u(x, y)=\tilde{u}(x, y)$ para todo $(x, y) \in O \backslash S$.

O próximo resultado caracteriza as funções cujas derivadas direcionais com relação a uma dada coordenada é nula. A notação $O_{x}$ será utilizada para denotar a secção vertical de $x$. isto $\dot{\mathrm{e}}$, dado $x \in \mathbb{R}^{M}, O_{x}:=\left\{y \in \mathbb{R}^{N} \mid(x, y) \in O\right\}$. Se $x \in \mathbb{R}^{M}$ e $\bar{y} \in \mathbb{R}^{n}, O_{x}(\bar{y})$ denotará a componente conexa de $\bar{y}$ em $O_{x}$.

Teorema 2.2.4 Suponhamos $M, N \in \mathbb{N}$. Sejam $O \subset \mathbb{R}^{M+N}$ um conjunto aberto e $u$ $L_{\text {loc }}^{1}(O)$. As seguintes afirmaçòes são equivalentes:

(1) $\nabla_{y} u=0$ no sentido ditribucional;

(2) existe um conjunto de medida nula $Z \subset \mathbb{R}^{M}$ e para todo $x \in \mathbb{R}^{M} \backslash Z$ existe um conjunto de medida nula $S_{x} \in \mathbb{R}^{N}$ tal que, sempre que $y \in O_{x}$, existe uma constante $v(x, \bar{y}) \in \mathbb{R}$ com a propriedade $u(x, y)=v(x, \bar{y})$ para todo $y \in O_{x}(\bar{y}) \backslash S_{x}$.

Demonstração. Suponhamos que (1) esteja verificada. Consideremos una cobertura de $O$ por uma família enumerável $\left(O_{n}\right)_{n \in \mathbb{N}}$ de conjuntos da forma $O_{n}=U_{n} \times V_{n}$, onde $U_{n}$ e $V_{n}$ são retângulos abertos limitados em $\mathbb{R}^{M}$ e $\mathbb{R}^{N}$ respectivamente. Notemos também que

$$
\nabla_{y}\left(\left.u\right|_{O_{n}}\right)=\left.\left(\nabla_{y} u\right)\right|_{O_{n}}=0 \text { para todo } n \in \mathbb{N}
$$

O Lema 2.2.2 implica que, para cada $n \in \mathbb{N}$, existe um conjunto de medida nula $S_{n} \in$ $\mathbb{R}^{M+N}$ e uma função $v_{n} \in L_{\text {loc }}^{1}\left(U_{n}\right)$ tal que $u(x, y)=v_{n}(x)$ sempre que $(x, y) \in O_{n} \backslash S_{n}$. Seja $S:=\bigcup_{n=1}^{\infty} S_{n}$. Observe que

$$
\mu_{M+N}(S)=\int_{\mathbb{R}^{M}} \mu_{N}\left(S_{x}\right) \mathrm{d} x=0 .
$$

Com isso, vemos que existe um conjunto de medida nula $Z \subset \mathbb{R}^{M}$ tal que, para todo $x \in \mathbb{R}^{M} \backslash Z, \mu_{N}\left(S_{x}\right)=0$. Agora, fixemos $(x, \bar{y}) \in O \operatorname{com} x \notin Z$. Então $(x, \bar{y}) \in O_{m}$ para $\operatorname{algum} m \in \mathbb{N}$.

Definamos $A$ como sendo o conjunto de todos os $z \in O_{\text {ir }}(y)$ para os quais existe um retângulo aberto $V:=V_{z} \subset O_{x}$ com $z \in V$, tal que $u(x, y)=v_{m}(x)$ para todo $y \in V \backslash S_{x}$. 
Notemos que $y \in A$. De fato, basta tomarmos $V=V_{m}$. Logo. $A \neq \emptyset$. O conjunto $A$ também é aberto em $O_{x}(\bar{y})$ já que, se $z_{0} \in A$ e $V=V_{z_{0}}$ é como na definição de $A$, então $V . \subset A$. Seja agora $z \in O_{x}(\bar{y}) \backslash A$. Então $(x, z) \in O_{k}$ para algum $k \in \mathbb{N}$. Segue que $u(x, y)=v_{k}(x)$ para todo $y \in V_{k} \backslash S_{x}$. Se $v_{k}(x)=v_{m}(x)$, então a definição do conjunto $A$ implica que $z \in A$, o que é absurdo. Logo $v_{k}(x) \neq v_{m}(x)$ e também $V_{k} \cap A \neq \emptyset$, caso contrário, $V_{k} \subset A$. Logo, $A$ é também fechado em $O_{x}(y)$. Da conexidade de $O_{x}(y)$ temos que $A=O_{x}(y)$.

Com isso, $O_{x}(\bar{y})=\bigcup_{z \in A} V_{z}$. Seja $y \in O_{x}(y) \backslash S_{x}$. Entāo $y \in V_{z}$ para algum $z \in A$ e assim $u(x, y)=v_{n}(x)$. Portanto, $u(x, y)=v_{m}(x)$ para todo $y \in O_{x}(y) \backslash S_{x}$. Assim, a propriedade (2) está satisfeita.

Reciprocamente, suponhamos que a condição (2) seja válida. Considere uma função arbitrária $\phi \in \mathcal{D}(O)$, com $K:=\operatorname{supp} o \subset O$.

Denotemos por $P$ a projeção canônica de $\mathbb{R}^{M+N}$ sobre $\mathbb{R}^{M}$. Segue que

$$
\int_{O} u(x, y) \nabla_{y} \phi(x, y) \mathrm{d} x \mathrm{~d} y=\int_{P(O) \backslash Z}\left(\int_{O_{x}} u(x, y) \nabla_{y} \phi(x, y) \mathrm{d} y\right) \mathrm{d} x
$$

Fixemos agora $x \in P(O) \backslash Z$. Se mostrarmos que

$$
\int_{O_{O_{x}}} u(x, y) \nabla_{y} \phi(x, y) \mathrm{d} y=0
$$

teremos finalizado a demonstração, pois (2.7) implica que, para toda função $\phi \in \mathcal{D}(O)$,

$$
\int_{0} \nabla_{y} u(x, y) \phi(x, y) \mathrm{d} y=\int_{0} u(x, y) \nabla_{y} \phi(x, y) \mathrm{d} y=0,
$$

ou seja, $\nabla_{y} u=0$. Mostremos que a igualdade em (2.7) é verificada.

O conjunto $K_{x}$ é compacto em $\mathbb{R}^{v}$ e $K_{x} \subset O_{x}$. Assim, existem componentes conexas $\omega_{1}, \cdots, \omega_{n}$ de $O_{x}$ luas a duas disjuntas tais que

$$
K_{x} \subset \omega_{1} \cap \ldots \cap \omega_{n}
$$

Como cada $\omega_{i}, i=1 \ldots, n$, é conexo, segue que cada $K_{x} \cap \omega_{i}$ é compacto, $i=1, \ldots, n$.

Por hipótese, existem numoros reais $c_{1}(x), \ldots, c_{n}(x)$ tais que $u(x, y)=c_{k}(x)$ para $y \in \omega_{k} \backslash S_{x}, k=1, \ldots, n$. Assim,

$$
\int_{O_{O_{x}}} u(x, y) \nabla_{y} \phi(x, y) \mathrm{d} y=\sum_{k=1}^{n} \int_{\omega_{k}} u(x, y) \nabla_{y} \phi(x, y) \mathrm{d} y=\sum_{k-1}^{n} c_{k}(x) \int_{\omega_{\omega_{k}}} \nabla_{y} \phi(x, y) \mathrm{d} y .
$$

Fixando $k=1, \ldots, n$ e definindo

$$
\alpha(y):= \begin{cases}\varphi(x, y), & y \in \omega_{k} \\ 0, & y \in \mathbb{R}^{N} \backslash \omega_{k} .\end{cases}
$$


temos que $\alpha \in \mathcal{D}\left(\mathbb{R}^{N}\right), \operatorname{supp} \alpha-K_{x} \cap \omega_{k}$ e

$$
\nabla \alpha(y):= \begin{cases}\nabla \phi(x, y), & y \in \omega_{k} \\ 0, & y \in \mathbb{R}^{N} \backslash \omega_{k} .\end{cases}
$$

Assim,

$$
\int_{\omega_{k}} \nabla_{y} \phi(x, y) \mathrm{d} y=\int_{\mathbb{K}^{*}} \nabla \alpha(y) \mathrm{d} y=0
$$

As igualdades (2.8) e (2.9) implicam que a igualdade cm (2.7) está verificada. O teorema está demonstrado.

Seja $O \subset \mathbb{R}^{i+N}$ um domínio não-vazio e limitado. Definimos

$$
H_{s}^{1}(O):=\left\{u \in H^{1}(O) \mid \nabla_{y} u=0\right\}
$$

Proposição 2.2.5 O conjunto $H_{s}^{1}(O)$ é um subespaço fechado de $H^{1}(O)$. Além disso, $I_{s}^{1}(O)$ possui dimensão infinita.

Demonstração. Notemos que $I_{s}^{1}(O)$ é claramente um subespaço de $I^{1}(O)$. Para mostrarmos que elé é fechado, seja $\left(u_{n}\right)_{n \in \mathbb{N}}$ uma sequência em $I_{s}^{1}(O) \operatorname{com} u_{n} \rightarrow u$ na norma de $H^{1}(O)$. Logo, para cada $i=1, \ldots, N$

$$
\left|\partial_{y_{i}} u_{n}-\partial_{y_{i}} u\right|_{L^{2}(O)} \rightarrow 0 \text { quando } n \rightarrow \infty
$$

Como $u_{n} \in H_{s}^{1}(O)$, temos que $\partial_{y_{i}} u_{n}=0, i=1 \ldots, N$. Logo, $\partial_{y_{i}} u=0, i=1 \ldots, N$ e portanto $u \in H_{s}^{1}(O)$.

Sejam $U$ e $V$ retângulos abertos, limitado e não vazios satisfazendo $U \subset \mathfrak{K}^{\text {ivi }}$ e $V \subset \mathbb{R}^{N}$ com $U \times V \subset O$. Vamos definir uma aplicação linear injetiva $\Gamma: \mathcal{D}(U) \rightarrow H_{s}^{1}(O)$. Como $\mathcal{D}(U)$ ć de dimensão infinita, $H_{s}^{1}(O)$ também o será. Dado $\phi \in \mathcal{D}(U)$, seja $\Gamma(\phi)=u$, definida por

$$
u(x, y):= \begin{cases}\phi(x), & \text { se } x \in U \\ 0, & \text { caso contrário }\end{cases}
$$

Temos que $u \in C^{\infty}(O)$,

e $\partial_{y_{\imath}} u=0$ para todo $i=1, \ldots, N$.

$$
\partial_{x_{i}} u= \begin{cases}\partial_{x_{i}} \phi, & \text { em } U, \\ 0, & \text { caso contrário }\end{cases}
$$

Notemos também que, como $O$ e limitado, $u, \partial_{x_{i}} u \in L^{2}(O), i=1, \ldots, N$. Uma aplicação do Tcorema 2.2.4 implica que $u \in H_{s}^{1}(O)$. Logo, $\Gamma: \mathcal{D}(U) \longrightarrow H_{s}^{1}(O)$ está bem definida e, é claro que, $\Gamma$ é uma aplicação linear. Notemos ainda que, se $\Gamma(\phi)=0$, entäo $\phi=0$, ou seja, $\Gamma$ é injetiva.

A Proposição 2.2 .5 implica que o espaço $H_{s}^{1}(O)$ é completo para o produto escalar herdado de $H^{1}(O)$ e, portanto, é também un espaço de Hilbert.

Definamos agora o espaço $L_{s}^{2}(O)$ com sendo o fecho de $H_{s}^{1}(O)$ em $L^{2}(O)$. Assim, $L_{s}^{2}(O)$ é também um espaço de Hilbert com o produto escalar herdado de $L^{2}(O)$. 


\subsection{O problema limite}

Vamos agora apresentar o problema limite para $\left(R D_{\varepsilon}\right), \varepsilon>0$. Utilizaremos a notação introduzida na Seção 2.1. Recordemos que $a_{\varepsilon}: I I^{1}(\Omega) \times I I^{1}(\Omega) \rightarrow \mathbb{R}$ ć a forma bilinear dada por

$$
a_{\varepsilon}(u, v):=\int_{\Omega}^{n}\left(\nabla_{x} u \cdot \nabla_{x} v+\frac{1}{\varepsilon^{2}} \nabla_{y} u \cdot \nabla_{y} v\right) \mathrm{d} x \mathrm{~d} y .
$$

Dada uma função $u \in H^{1}(\Omega)$, é fácil ver que

$$
\lim _{\varepsilon \rightarrow 0^{+}} a_{\Xi}(u, u)=\int_{\Omega}^{n} \nabla_{x} u \cdot \nabla_{x} u \mathrm{~d} x \mathrm{~d} y, \text { se } \nabla_{y} u=0
$$

e que

$$
\lim _{\varepsilon \rightarrow 0^{+}} a_{\varepsilon}(u, u)=\infty, \text { se } \nabla_{y} u \neq 0 .
$$

Vamos definir uma forma bilinear $a_{0}$ em $I_{s}^{1}(\Omega)$. Esta scrá a forma bilinear "limite" que nos dará o semifluxo "limite" para a família de semifluxos $\left(\pi_{\varepsilon}\right)_{\varepsilon>0}$.

Definimos $a_{0}: H_{s}^{1}(\Omega) \times H_{s}^{1}(\Omega) \rightarrow \mathbb{R}$ por

$$
a_{0}(u, v):=\int_{J_{\Omega}} \nabla u \cdot \nabla v \mathrm{~d} x \mathrm{~d} y
$$

A definição de $H_{s}^{1}(\Omega)$ implica que

$$
a_{0}(u, v)=\int_{\Omega} \nabla_{x} u \cdot \nabla_{x} v \mathrm{~d} x \mathrm{~d} y \text {, para quaisquer que sejam } u, v \in H_{s}^{1}(\Omega) .
$$

Em $i_{s}^{2}(j i)$ estamos considerando o produto interno dado por

$$
\langle u, v\rangle_{L_{s}^{2}(\Omega)}:=\int_{\Omega} u v \mathrm{~d} x \mathrm{~d} y .
$$

Seja $b_{0}$ a restrição de $\langle\cdot, \cdot\rangle_{L_{s}^{2}(\Omega)}$ a $H_{s}^{1}(\Omega) \times H_{s}^{1}(\Omega)$. Do mesmo modo que para o caso $a_{\hat{c}}$, temos que $a_{0}$ é contínua e coerciva. Sendo assim, as hipóteses da Proposição 1.1.2 estão satisfeitas para o par $\left(a_{0}, b_{0}\right)$. Também estão satisfeitas as hipóteses das Proposições 1.1.7 e 1.1.8. Estas proposições implicam no seguinte resultado:

Lema 2.3.1 O conjunto dos autovalores de $\left(a_{0}, b_{0}\right)$ é infinito e enumerável, possui um menor elemento e cada autovalor possui multiplicidade finita. Além disso, o par $\left(a_{0}, b_{0}\right)$ gera um operador setorial $A_{0}$ em $X=L_{s}^{2}(\Omega)$. O espaço de potências fracionárias associado a $\alpha=1 / 2$ satisfaz $X^{\alpha}=H_{s}^{1}(\Omega)$.

Queremos agora mostrar que o operador $\hat{f}$ definido na Seção 2.1 associado a função $f$ aplica $H_{s}^{1}(\Omega)$ em $L_{s}^{2}(\Omega)$. Antes porém, temos o seguinte resultado, o qual é uma adaptação do Corolário IX.5 de [3]. 
Lema 2.3.2 Seja $G \in C^{1}(\mathbb{R})$ uma função satisfazendo $\sup _{s \in \mathbb{R}}\left|G^{\prime}(s)\right|<\infty$. Se $p \geq 1$ e $u \in W^{1, p}(\Omega)$, então $G \circ u \in W^{1, p}(\Omega)$ e $\nabla(G \circ u)=\left(G^{\prime} \circ u\right) \nabla u$.

Demonstração. Como $\sup _{s \in \mathbb{R}}\left|G^{\prime}(s)\right|<\infty$ e $\mu(\Omega)<\infty$, temos que existe uma constante $M>0$ tal que $|G(s)| \leq \bar{M}|s|$ para todo $s \in \mathbb{R}$. Assim,

$$
\int_{\Omega}|G \circ u|^{p} \mathrm{~d} x \mathrm{~d} y \leq M^{p} \int_{J_{\Omega}}|u|^{p} \mathrm{~d} x \mathrm{~d} y<\infty
$$

ou seja, $G \circ u \in L^{p}(\Omega)$. A limitação de $G^{\prime}$ também implica que $\left(G^{\prime} \circ u\right) \partial u \in L^{p}(\Omega)$. Para completar a demonstração, falta mostrar que

$$
\int_{\Omega}(G \circ u) \partial \psi \mathrm{d} x \mathrm{~d} y=-\int_{\Omega}\left(G^{\prime} \circ u\right) \partial u \psi \mathrm{d} x \mathrm{~d} y, \text { qualquer que seja } \psi \in \mathcal{D}(\Omega) .
$$

Seja $\psi \cdot \in \mathcal{D}(\Omega)$. Vamos considerar dois casos

Caso 1. Seja $1 \leq p<\infty$. Segue do Teorema IX.2 em [3] que existe uma sequência $\left(u_{n}\right)_{n \in \mathbb{N}}$ em $\mathcal{D}(\Omega)$ tal que $u_{n} \rightarrow u$ em $L^{p}(\Omega)$ quase sempre em $\Omega$ e, se $\omega \subset \subset \Omega$, então $\partial u_{n} \rightarrow \partial u$ em $I^{\nu}(\omega)$. Alóm clisso,

$$
\int_{\Omega}^{r}\left(G \circ u_{n}\right) \partial \psi \mathrm{d} x \mathrm{~d} y=-\int_{\Omega}^{r}\left(G^{\prime} \circ u_{n}\right) \partial u_{n} \psi \mathrm{d} x \mathrm{~d} y \text { qualquer que seja } \psi \in \mathcal{D}(\Omega) \text {. }
$$

Como $G \circ u_{n} \rightarrow G \circ u$ em $L^{p}(\Omega)$, o Teorema da Convergência Dominada de Lebesgue implica que $\left(G^{\prime} \circ u_{n}\right) \partial u_{n} \rightarrow\left(G^{\prime} \circ u\right) \partial u$. Assim, fazendo $n \rightarrow \infty$ em (2.11) obtemos (2.10).

Caso 2. Seja $p=\infty$ e $\Omega_{\psi}$ um aberto tal que supp $\psi \subset \Omega_{\psi} \subset \subset \Omega$. Portanto $u \in W^{1, p}\left(\Omega_{\psi}\right)$ para todo $p<\infty$, e pelo Caso 1 ,

$$
\begin{aligned}
\int_{\Omega}(G \circ u) \partial \psi \mathrm{d} x \mathrm{~d} y & =\int_{\Omega_{\psi}}^{n}(G \circ u) \partial \psi \mathrm{d} x \mathrm{~d} y \\
& =-\int_{\Omega_{\psi}}^{f}\left(G^{\prime} \circ u\right) \partial u \psi \mathrm{d} x \mathrm{~d} y=-\int_{\Omega}\left(G^{\prime} \circ u\right) \partial u \psi \mathrm{d} x \mathrm{~d} y
\end{aligned}
$$

qualquer que seja $\psi \in \mathcal{D}(\Omega)$. A demonstração está completa.

Teorema 2.3.3 Seja $g \in C^{\prime 1}(\mathbb{R})$ e $u \in H_{s}^{1}(\Omega)$ tal que $g \circ u \in L^{2}(\Omega)$. Então gou $\in L_{s}^{2}(\Omega)$.

Demonstração. Dado $n \in \mathbb{N}$, seja $h_{n} \in C_{0}^{1}(\mathbb{R})$ tal que $0 \leq h_{n} \leq 1$ e $h_{n}(s)=1$ para $|s| \leq n$. Para cada $n \in \mathbb{N}$, definimos $g_{n}:=g h_{n}$. Segue que $g_{n} \in C_{0}^{1}(\mathbb{R})$ para todo $n \in \mathbb{N}$. Assim, para cada $n \in \mathbb{N}$, $\sup _{s \in \mathbb{R}}\left|q_{n}^{\prime}(s)\right|<\infty$. Portanto, pelo Lema 2.3.2 temos que $g_{n} \circ u \in H^{1}(\Omega)$ e $\nabla_{y}\left(g_{n} \circ u\right)=\left(g_{n}^{\prime} \circ u\right) \nabla_{y} u$. Como $\nabla_{y} u=0$, temos que $\nabla_{y}\left(g_{n} \circ u\right)=0$ para todo $n \in \mathbb{N}$. Logo, $g_{n} \circ u \in H_{s}^{1}(\Omega)$ para todo $n \in \mathbb{N}$.

Como $u(x, y)$ é finito para quase todo $(x, y) \in \Omega$, segue que existe um $n_{0}=n_{0}(x, y)$ com $|u(x, y)| \leq n_{0}$ para quase todo $(x, y) \in \Omega$. A definição de $g_{n}$ implica que $\mid g_{n} \circ u(x, y)-$ 
$g \circ u(x, y) \mid=0$ para todo $n \geq n_{0}$. Logo, $\left|g_{n} \circ u(x, y)-g \circ u(x, y)\right| \rightarrow 0$ para quase todo $(x, y) \in \Omega$. Notemos que $\left|g_{n} \circ u\right| \leq|g| \circ u$ e, portanto, $\left|g_{n} \circ u-g \circ u\right| \leq 2|g| \circ u$ para todo $n \in \mathbb{N}$. Como $|g| \circ u \in L^{2}(\Omega)$, segue do Teorema da Convergência Dominada de Lebesgue que $g_{n} \circ u \rightarrow g \circ u$ em $L^{2}(\Omega)$, ou seja, $g \circ u \in L_{s}^{2}(\Omega)$. O teorema está demonstrado.

O Teorema 2.3.3 implica, em particular, que $\hat{f}\left(H_{s}^{1}(\Omega)\right) \subset L_{s}^{2}(\Omega)$. Com isso, temos definido a seguinte equação de evolução:

$$
\dot{u}+A_{0} u=\hat{\jmath}(u) .
$$

O Lema 2.3.1 e o Teorema 2.1.8 juntamente com o Exemplo 1.2 .2 implicam que a equação $\left(R D_{0}\right)$ gera um semifluxo $\pi_{0}$ em $H_{s}^{1}(\Omega)$. Este será o semifluxo limite para a farnília $\left(\pi_{s}\right)_{s}>0$.

Queremos tornar mais precisa a idéia de como $\pi_{0}$ e $\left(\pi_{\varepsilon}\right)_{\varepsilon>0}$ estão relacionados. O primeiro passo é saber se $\left(\pi_{\varepsilon}\right)_{s>0}$ se aproxima, em algum sentido, do semifluxo $\pi_{0}$. Para realizarmos tal análise vamos introduzir, para cada $\varepsilon>0$, uma norma em $H^{1}(\Omega)$.

Para cada $\varepsilon>0$, definimos a norma $|\cdot|_{\varepsilon} \operatorname{em~} H^{1}(\Omega)$ por:

$$
|u|_{\varepsilon}:=\left(a_{\varepsilon}(u, u)+|u|_{L^{2}(\Omega)}^{2}\right)^{1 / 2}
$$

Proposição 2.3.4 Para cada $\varepsilon>0$, as normas $|\cdot|=e|\cdot|_{H^{1}(\Omega)}$ são equivalentes

Demonstração. Notemos que, para toda função $u \in H^{1}(\Omega)$, se $0<\varepsilon \leq 1$ vale

$$
|u|_{H^{1}(\Omega)} \leq|u|_{\Sigma} \leq \frac{1}{c^{2}}|u|_{H^{1}(\Omega)}
$$

e se $\varepsilon>1$,

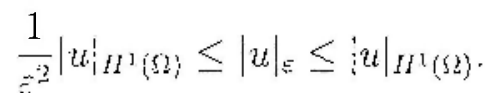

Isso completa a demonstração

Observemos que $|u|_{\varepsilon} \rightarrow \infty$ quándo $\varepsilon \rightarrow 0^{+}$sempre que $u \in H^{1}(\Omega)$ é tal que $\nabla_{y} u \neq 0$ em $L^{2}(\Omega)$, pois nesse caso, $\lim _{\varepsilon, 0^{+}} a_{\varepsilon}(u, u)=\infty$. Por outro lado, se $u \in H^{1}(\Omega)$, temos que $|u|_{\varepsilon}=|u|_{H^{1}(\Omega)}$, ou seja, a família $\left(|u|_{\varepsilon}\right)_{\varepsilon>0}$ possui limite finito quando $\varepsilon \rightarrow 0^{+}$.

Concluímos a seção com o lema que nos auxiliará na demonstração do resultado sobre convergência espectral a ser analisado no próximo capítulo.

Proposição 2.3.5 Seja $\left(\varepsilon_{n}\right)_{n \in \mathbb{N}}$ um sequênciu de números reais positivos com $\varepsilon_{n} \rightarrow 0$, quando $n \rightarrow \infty$. Suponhamos que $\left(u_{n}\right)_{n \in \mathbb{N}}$ seja uma sequência em $H^{1}(\Omega)$ e que existe um $u \in H_{s}^{1}(\Omega)$ tal que

$$
\left|u_{n}-u\right|_{\varepsilon_{n}} \rightarrow 0 \text { quando } n \rightarrow \infty
$$

As seguintes propriedades são verificadas:

(1) $u_{n} \rightarrow u e m H^{1}(\Omega)$ quando $n \rightarrow \infty$; 
(2) $\left|a_{\varepsilon_{n}}\left(u_{n}, u_{n}\right)-a_{0}(u, u)\right| \rightarrow 0$ quando $n \rightarrow \infty$.

Demonstração. Como $\varepsilon_{n} \rightarrow 0$ quando $n \rightarrow \infty$, existe um $n_{0} \in \mathbb{N}$ tal que $\varepsilon_{n} \leq 1$ para todo $n \geq n_{0}$. A Proposição 2.3.4 implica que

$|u|_{H^{1}(\Omega)} \leq|u|_{\varepsilon_{n}}$ qualquer que seja $u \in H^{1}(\Omega)$ e para todo $n \geq n_{0}$.

Em particular,

$$
\left|u_{n}-u\right|_{H^{1}(\Omega)} \leq\left|u_{n}-u\right|_{\varepsilon_{n}} \text { para todo } n \geq n_{0} .
$$

A desigualdade acima juntamente com (2.12) demonstram a afirmativa (1).

Para provar (2), primeiro observemos que, se $u \in H_{*}^{1}(\Omega)$, para todo $\varepsilon>0$ temos que

$$
|u|_{\Xi}^{2}=\int_{\Omega}\left(\nabla_{x} u \cdot \nabla_{x} u\right) \mathrm{d} x \mathrm{~d} y+|u|_{L^{2}(\Omega)}^{2}=a_{0}(u, u)+|u|_{L^{2}(\Omega)}^{2} .
$$

Portanto,

$$
\begin{aligned}
\left|a_{\varepsilon_{n}}\left(u_{n}, u_{n}\right)-a_{0}(u, u)\right| & \leq\left.\left|a_{\varepsilon_{n}}\left(u_{n}, u_{n}\right)+\right| u_{n}\right|_{I^{2}(\Omega)} ^{2}-a_{0}(u, u)-|u|_{L^{2}(\Omega)}^{2} \mid \\
& +\left.|| u_{n}\right|_{L^{2}(\Omega)} ^{2}-|u|_{L^{2}(\Omega)}^{2} \mid \\
& =\left|j u u_{n}\right|_{\varepsilon_{n}}^{2}-|u|_{\varepsilon_{n}}^{2}|+|\left|u_{n}\right|_{L^{2}(\Omega)}^{2}-|u|_{L^{2}(\Omega)}^{2} \mid \\
& =\left.|| u_{r}\right|_{\varepsilon_{n}} ^{2}-|u|_{H^{1}(\Omega)}^{2}|+|\left|u_{n}\right|_{L^{2}(\Omega)}^{2}-|u|_{L^{2}(\Omega)}^{2} \mid .
\end{aligned}
$$

Mas,

$$
\left.|| u_{n}\right|_{\varepsilon_{n}}-|u|_{\xi_{n}}|\leq| u_{n}-\left.u\right|_{\varepsilon_{n}} \rightarrow 0, \text { quando } n \rightarrow \infty .
$$

Como $|u|_{\Sigma_{n}}=|u|_{H^{1}(\Omega)}$ para todo $n \in \mathbb{N}$, temos que $\left|u_{n}\right|_{\varepsilon_{n}} \rightarrow|u|_{H^{1}(\Omega)}$ quando $n \rightarrow \infty \mathrm{e}$ portanto, $\left.\left.\right|_{i u}\right|_{s_{s,}} ^{2} \rightarrow|u|_{H^{2}(\Omega)}^{2}$ quando $n \rightarrow \infty$. Segue da desigualdade

$$
\left.|| u_{n}\right|_{L^{2}(\Omega)}-|u|_{L^{2}(\Omega)}|\leq| u_{n}-\left.u\right|_{H^{1}(\Omega)} \leq\left|u_{n}-u\right|_{\xi_{n}}
$$

que $\left|u_{n}\right|_{L^{2}(\Omega)}^{2} \rightarrow|u|_{L^{2}(\Omega)}^{2}$

Com isso,

$$
\left|a_{\varepsilon_{n}}\left(u_{n}, u_{n}\right)-a_{0}(u, u)\right| \rightarrow 0, \text { quando } n \rightarrow \infty .
$$

A demonstração está concluída. 


\section{Capítulo 3}

\section{Convergência Espectral}

Nesse capítulo vamos analisar o comportamento da família de autovalores e autovetores associados aos pares $\left(a_{\varepsilon}, b\right), \varepsilon>0$, quando $\varepsilon \rightarrow 0^{+}$. A Seção 3.1 apresenta o resultado de convergência desses autovalores e autovetores. Sob certas condiçöes no domínio, ó possível obter uma propriedade de monotonicidade. Tal result ado é apresentado na Seção 3.2. Nesse capítulo utilizaremos a notação aprescntarla no Capítulo 2.

\subsection{Convergência espectral}

Para cada $\varepsilon>0$, o par $\left(a_{\varepsilon}, b\right)$ definido na Seção 2.1 satisfaz todas as hipótcscs das Proposições 1.1.2 e 1.1.3. Denotemos por $\left(\lambda_{\varepsilon, j}\right)_{j \in \mathbb{N}}$ a sequência repetida de autovalores do par $\left(a_{*}, b\right)$ e por $\left(u_{i, j}\right)_{j=\mathbb{N}}$ a sequência de autovetores $L^{2}(\Omega)$-ortonormal completa correspondente. Segue da Proposiçĩo 1.1.2 que, para cada $\varepsilon>0$

$$
\lambda_{\varepsilon, 1} \leq \lambda_{\varepsilon, 2} \leq \lambda_{\varepsilon, 3} \leq \cdots .
$$

Notemos ainda que o par $\left(a_{0}, b_{0}\right)$ definido na Scção 2.3 também satisfaz as hipótcses

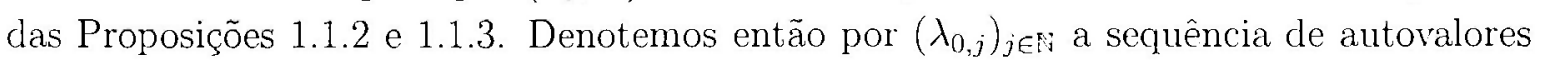
de $\left(n: 1, b_{0}\right)$. Segue que

$$
\lambda_{0,1} \leq \lambda_{0,2} \leq \lambda_{0.3} \leq \cdots .
$$

O próximo resultado nos mostra como, para cada $j \in \mathbb{N}$, as famílias $\left(\lambda_{\varepsilon, j}\right)_{\varepsilon>0}$ e $\left(w_{\varepsilon, j}\right)_{\varepsilon>0}$ se comportam quando $\varepsilon \rightarrow 0^{+}$. No caso da família $\left(w_{z, j}\right)_{\varepsilon>0}$, somente conseguiremos obter alguma resposta se considerarmos a norma $|\cdot|_{\varepsilon}$ introduzida na Seção 2.3. Temos então o resultado mais importante deste capítulo:

Teorema 3.1.1 Com a notação introduzida acima, valem as seguintes propriedades:

(1) para todo $j \in \mathbb{N}$, se $\varepsilon_{1}<\varepsilon_{2}$, entäo $\lambda_{\varepsilon_{1}, j} \geq \lambda_{\varepsilon_{2}, j}$. Além disso, para cada $j \in \mathbb{N}$,

$$
\lambda_{0, j}=\lim _{\varepsilon \rightarrow 0^{+}} \lambda_{\varepsilon, j}=\sup _{\varepsilon>0} \lambda_{\varepsilon, j} ;
$$


(2) $\operatorname{seja}\left(\varepsilon_{n}\right)_{n \in \mathbb{N}}$ uma sequência arbitrária de números positivos com $\varepsilon_{n} \rightarrow 0$ quando $n \rightarrow \infty$. Então existe uma subsequência de $\left(\varepsilon_{n}\right)_{n \in \mathbb{N}}$, denotada novamente por $\left(\tau_{n}\right)_{n \in \mathbb{N}}$, e existe um sistema $L_{s}^{2}(\Omega)$-ortonormal $\left(w_{0, j}\right)_{j \in \mathbb{N}}$ de autovetores de $\left(a_{0}, b_{0}\right)$ correspondendo à sequência de autovalores $\left(\lambda_{0, j}\right)_{j \in \mathbb{N}}$ tais que, para todo $\jmath \in \mathbb{N}$,

$$
\left|u_{\varepsilon_{n}, j}-w_{0, j}\right|_{\varepsilon_{n}} \rightarrow 0 \text { quando } n \rightarrow \infty
$$

Demonstração. Nesta demonstração utilizaremos a notação $\langle u, v\rangle$ para representar $\langle u, v\rangle_{L^{2}(\Omega)}$. Seja $\varepsilon>0$ e $j \in \mathbb{N}$. Pela Proposição 1.1.3 temos que

$$
\lambda_{\Sigma, j}=\min _{i \cdot \in \mathcal{V}_{j}} \max _{u \in F \backslash\{0\}} \frac{a_{\varepsilon}(u, u)}{\langle u, u\rangle},
$$

onde $\mathcal{V}_{j}$ é o conjunto de todos os subespaços $j$-dimensionais de $H^{1}(\Omega)$.

Sejam $\varepsilon_{1}, \varepsilon_{2}$ tais que $0<\varepsilon_{1}<\varepsilon_{2}$. Com isso, $1 / \varepsilon_{1}^{2}>1 / \varepsilon_{2}^{2}$ e, portanto, se $u \in H^{1}(\Omega)$,

$$
a_{\varepsilon_{1}}(u, u) \geq a_{\varepsilon_{2}}(u, u) .
$$

Assim, (3.1) e (3.2) implicam que $\lambda_{\Sigma_{1}, \jmath} \geq \lambda_{E_{2, j} .}$. Além disso, como $b_{0}$ é a restrição de $\langle\cdot, \cdot\rangle$ a $H_{s}^{1}(\Omega) \times H_{s}^{1}(\Omega)$, segue da Proposição 1.1.3 que, para cada $j \in \mathbb{N}$,

$$
\lambda_{0 . j}=\min _{F \in \mathcal{U}_{j}} \max _{u \in F \backslash\{0\}} \frac{a_{0}(u, u)}{\langle u, u\rangle},
$$

onde $\mathcal{U}_{j}$ é o conjunto de todos os subespaços $j$-dimensionais de $H_{s}^{1}(\Omega)$. Observemos que $H_{s}^{1}(\Omega) \subset H^{1}(\Omega)$ e que $a_{\bar{s}}(u, u)=a_{0}(u, u)$ para toda função $u \in H_{s}^{1}(\Omega)$ e para todo $\varepsilon>0$. Assim, $\mathcal{U}_{j} \subset \mathcal{V}_{j}$ para cada $j \in \mathbb{N}$. Seja $E \in \mathcal{U}_{j}$. Logo, $E \in \mathcal{V}_{j}$. Tomando $u \in E$ com $u \neq 0$, temos que

$$
\frac{a_{\varepsilon}(u, u)}{\langle u, u\rangle}=\frac{a_{0}(u, u)}{\langle u, u\rangle}
$$

Com isso,

$$
\min _{i \in \mathcal{V}_{j}} \max _{u \in F \backslash\{0\}} \frac{a(\{, u)}{\langle u, u\rangle} \leq \min _{l, \in \mathcal{U}_{j}} \max _{u \in E \backslash\{0\}} \frac{a_{0}(u, u)}{\langle u, u\rangle}
$$

isto é, $\lambda_{\varepsilon, j} \leq \lambda_{0, j}$, para todo $j \in \mathbb{N}$ e todo $\varepsilon>0$. Assim, para cada $j \in \mathbb{N}$, a família $\left(\lambda_{\varepsilon, j}\right)_{\Sigma>0}$ é limitada superiormente. Este fato e a desigualdade (3.2) implicam que, para cada $j \in \mathbb{N}$, existe um $\mu_{j} \in \mathbb{R}$ tal que

$$
\mu_{j}=\lim _{\varepsilon \rightarrow 0} \lambda_{\varepsilon, j}=\sup _{\varepsilon>0} \lambda_{\varepsilon, j}
$$

Observemos ainda que $\mu_{j}<\lambda_{0, j}$ e que, para $j_{1}<j_{2}$, segue que $\mu_{j_{i}} \leq \mu_{j_{2}}$.

Nosso objetivo é mostrar que, para todo $\jmath \in \mathbb{N}, \mu_{j}=\lambda_{0,3}$ e também construir uma sequência $\left(w_{0, j}\right)_{j \in \mathbb{N}}$ Com as propriedades descritas em (2). Seja então $\left(\varepsilon_{n}\right)_{n \in \mathbb{N}}$ uma sccuência de números positivos com $\varepsilon_{n} \rightarrow 0$, quando $n \rightarrow \infty$. Podemos assumir som 
perda de generalidade que $\varepsilon_{n}<1$, para todo $n \in \mathbb{N}$. Fixemos $j \in \mathbb{N}$. Como para cada $n \in \mathbb{N},\left(w_{\varepsilon_{n}, j}\right)_{j \in \mathbb{N}}$ é um sistema $L^{2}(\Omega)$-ortonormal e $w_{\varepsilon_{n}, j}$ é um autovetor associado a $\lambda_{\varepsilon_{n}, j}$ temos que

$$
a_{\varepsilon_{n}}\left(w_{\varepsilon_{n}, j}, w_{\varepsilon_{n}, j}\right)=\lambda_{\varepsilon_{n}, j}\left\langle w_{\varepsilon_{n}, j}, w_{\varepsilon_{n}, j}\right\rangle=\lambda_{\varepsilon_{n}, j}, \text { para todo } n \in \mathbb{N}
$$

Taunbém,

$$
a_{\varepsilon_{n}}\left(w_{\varepsilon_{n}, j}, w\right)=\lambda_{\varepsilon_{n}, j}\left\langle w_{\varepsilon_{n}, j}, w\right\rangle, \text { qualquer que seja } w \in H^{1}(\Omega) \text { e todo } n \in \mathbb{N} \text {. }
$$

Consequentemente,

$$
\begin{aligned}
\left|w_{\varepsilon_{n}, j}\right|_{I^{1}(\Omega)}^{2} & \leq \int_{\Omega} \nabla_{x} w_{\varepsilon_{n}, j} \cdot \nabla_{x} w_{\varepsilon_{n}, j} \mathrm{~d} x \mathrm{~d} y+\frac{1}{\varepsilon_{n}^{2}} \int_{\Omega} \nabla_{y} w_{\varepsilon_{n}, j} \cdot \nabla_{y} w_{\varepsilon_{n}, j} \mathrm{~d} x \mathrm{~d} y+\left|w_{\varepsilon_{n}, j}\right|_{L^{2}(\Omega)}^{2} \\
& =a_{\varepsilon_{n}}\left(w_{\varepsilon_{n}, j}, w_{\varepsilon_{n}, j}\right)+1=\lambda_{\varepsilon_{n}, j}+1 \leq \lambda_{0, j}+1 .
\end{aligned}
$$

Assim, para cada $j \in \mathbb{N},\left(w_{\varepsilon_{n}, j}\right)_{n \in \mathbb{N}}$ é uma sequência limitada no espaço reflexivo $H^{1}(\Omega)$. Logo. existe uma subsequência de $\left(\dot{c}_{n}\right)_{n \in \mathbb{N}}$, que será denotada novamente por $\left(c_{n}\right)_{n \in N}$, e uma função $w_{0, j} \in H^{1}(\Omega)$ tal que

$$
w_{\Sigma_{n}, j} \rightarrow w_{0, j} \operatorname{em~} H^{1}(\Omega) .
$$

Como $\Omega$ possui fronteira Lipschitz, temos que $H^{1}(\Omega)$ está compactamente imerso em $L^{2}(\Omega)$. Logo

$$
w_{\varepsilon_{n}, j} \rightarrow w_{0, j} \operatorname{ern} I^{2}(\Omega)
$$

Em particular, como $\left|w_{\varepsilon_{n}, j}\right|_{L^{2}(\Omega)}=1$ para todo $n \in \mathbb{N}$, segue que $\left|w_{0, j}\right|_{L^{2}(\Omega)}=1$. Vamos mostrar que $w_{0, j} \in H_{s}^{1}(\Omega)$ para todo $j \in \mathbb{N}$.

Seja $j \in \mathbb{N}$. Observemos que

$$
a_{\varepsilon_{n}}\left(w_{\varepsilon_{n}, j}, w_{\varepsilon_{n}, j}\right) \geq \frac{1}{\varepsilon_{n}^{2}} \int_{\Omega l} \nabla_{y} w_{\varepsilon_{n}, j} \cdot \nabla_{y} w_{\varepsilon_{n}, j} \mathrm{~d} x \mathrm{~d} y \text { para todo } n \in \mathbb{N} \text {. }
$$

Portanto,

$$
\frac{1}{\varepsilon_{n}^{2}} \int_{\Omega} \nabla_{y} w_{\varepsilon_{n}, j} \cdot \nabla_{y} w_{\varepsilon_{n}, j} \mathrm{~d} x \mathrm{~d} y \leq \lambda_{\varepsilon_{n}, j} \leq \lambda_{0, j} \text { para todo } n \in \mathbb{N} .
$$

Temos então que

$$
\int_{\Omega} \nabla_{y} w_{\varepsilon_{n, j}} \cdot \nabla_{y} w_{\varepsilon_{n}, j} \mathrm{~d} x \mathrm{~d} y \rightarrow 0 \text { quando } n \rightarrow \infty .
$$

Logo, $\partial_{y_{1}} w_{\varepsilon_{n}, j} \rightarrow 0 \mathrm{em} L^{2}(\Omega)$, para cada $i=1, \ldots, N$.

Agora, fixemos $i=1, \ldots, N$. Para toda função $\phi \in \mathcal{D}(\Omega)$, segue da Desigualdade de Hölder que

$$
\int_{J \Omega}\left|\phi \partial_{y_{i}} w_{\varepsilon_{n}, j}\right| \mathrm{d} x \mathrm{~d} y \leq|\phi|_{L^{2}(\Omega)}\left|\partial_{y_{i}} w_{\varepsilon_{n}, j}\right|_{L^{2}(\Omega)}
$$


Assim, $\phi \partial_{y_{2}} w_{\varepsilon_{n}, j} \rightarrow 0$ em $L^{1}(\Omega)$ quando $n \rightarrow \infty$. Além disso,

$$
\int_{\Omega}\left|w_{\xi_{n}, j} \partial_{y_{\ell}} \phi-w_{0, j} \partial_{y_{i}} \phi\right| \mathrm{d} x \mathrm{~d} y \leq\left|w_{\varepsilon_{r, j}}-w_{0, j}\right|_{\mathcal{L}^{2}(\Omega)}\left|\partial_{y_{i}} \dot{\phi}\right|_{\ell^{2}(\Omega)}
$$

Como $w_{\varepsilon_{n}, \jmath} \rightarrow w_{0, j}$ em $L^{2}(\Omega)$, témos que $w_{\varepsilon_{n}, j} \partial_{y_{l}} \phi \rightarrow w_{0, j} \partial_{y_{l}} \phi$ em $\dot{L}^{-1}(\widehat{\jmath} \hat{\iota})$ qualquer que seja $\phi \in \mathcal{D}(\Omega)$. Por outro lado, para todo $n \in \mathbb{N}$,

$$
\int_{\Omega} \phi \partial_{y_{i}} w_{\varepsilon_{n}, j} \mathrm{~d} x \mathrm{~d} y=-\int_{\Omega} w_{\varepsilon_{n}, j} \partial_{y_{\imath}} \phi \mathrm{d} x \mathrm{~d} y
$$

Sendo assim,

$$
\begin{aligned}
\int_{\Omega_{\Omega}} w_{0, j} \partial_{y_{i}} \phi \mathrm{d} x \mathrm{~d} y & =\int_{\Omega \Omega}^{n} w_{0, j} \partial_{y_{i}} \phi \mathrm{d} x \mathrm{~d} y+\int_{\Omega_{\Omega}} \phi \partial_{y_{i}} w_{\varepsilon_{n}, j} \mathrm{~d} x \mathrm{~d} y-\int_{\Omega} \phi \partial_{y_{i}} w_{\varepsilon_{n}, j} \mathrm{~d} x \mathrm{~d} y \\
& =\int_{\Omega \Omega}^{n} w_{0, j} \partial_{y_{i}} \phi \mathrm{d} x \mathrm{~d} y-\int_{\Omega}^{n} w_{\varepsilon_{n}, j} \partial_{y_{i}} \phi \mathrm{d} x \mathrm{~d} y-\int_{\Omega} \phi \partial_{y_{i}} w_{\varepsilon_{n}, j} \mathrm{~d} x \mathrm{~d} y \\
& =\int_{\Omega}\left(w_{0, j} \partial_{y_{i}} \phi-w_{\varepsilon_{n}, j} \partial_{y_{i}} \phi\right) \mathrm{d} x \mathrm{~d} y-\int_{\Omega} \phi \partial_{y_{i}} w_{\varepsilon_{n}, j} \mathrm{~d} x \mathrm{~d} y .
\end{aligned}
$$

Estimando o módulo da expressão acima obtenos

$$
\left|\int_{\Omega} w_{0, j} \partial_{y_{i}} \phi \mathrm{d} x \mathrm{~d} y\right| \leq\left|w_{0, j} \partial_{y_{i}} \phi-w_{s_{n, j}} \partial_{y_{i}} \phi\right|_{L^{1}(\Omega)}+\left|\phi \partial_{x_{i}} w_{z_{2, j}, j}\right|_{L^{1}\{\Omega\}}
$$

Fazendo $n \rightarrow \infty$, obtemos que:

$$
\int_{\Omega}^{r} w_{0, j} \partial_{y_{i}} \phi \mathrm{d} x \mathrm{~d} y=0 \text { qualquer que seja } \phi \in \mathcal{D}(\Omega)
$$

ou seja, mostramos que $\partial_{y_{i}} w_{0, j}=0$, para cada $i=1, \ldots, N$. Portanto, $w_{0, j} \in H_{s}^{1}(\Omega)$ para todo $j \in \mathbb{N}$.

Afirmamos que $\left(\mu_{j}, w_{0, i}\right)_{i \in \mathbb{N}}$ é uma sequência de pares de autovalores-autovetores de $\left(a_{0}, b_{0}\right)$, isto é, para todo $j \in \mathbb{N}, a_{0}\left(w_{0, j}, w\right)=\mu_{j} b_{0}\left(w_{0, j}, w\right)$ qualquer que seja $w \in H_{s}^{1}(\Omega)$. De fato, seja $w \in H_{s}^{1}(\Omega)$ uma função arbitrária e fixemos $j \in \mathbb{N}$. Então:

$$
\begin{aligned}
\int_{\Omega} \nabla w_{\varepsilon_{n}, j} \cdot \nabla w \mathrm{~d} x \mathrm{~d} y & =\int_{\Omega} \nabla_{x} w_{\varepsilon_{n}, j} \cdot \nabla_{x} w \mathrm{~d} x \mathrm{~d} y \\
& =a_{\varepsilon_{\varepsilon_{n}}}\left(w_{\varepsilon_{n}, j}, w\right)=\lambda_{\varepsilon_{n}, j}\left\langle w_{\varepsilon_{n}, j}, w\right\rangle .
\end{aligned}
$$

Como $w_{\xi_{n, j}} \rightarrow w_{0, j}$ em $I^{1}(\Omega), \lambda_{\varepsilon_{n}, j} \rightarrow \mu_{j}$ e $w_{\varepsilon_{n, j}} \rightarrow w_{0, j}$ em $L^{2}(\Omega)$ quando $n \rightarrow \infty$, obtemos que

$$
a_{0}\left(w_{0, j}, w\right)=\int_{\Omega} \nabla w_{0, j} \cdot \nabla w \mathrm{~d} x \mathrm{~d} y=\mu_{j}\left\langle u_{0, j}, w\right\rangle=\mu_{j} b_{0}\left(w_{0, j}, w\right)
$$

o que demonstra nossa afirmação. 
Mostremos agora que, para cada $j \in \mathbb{N},\left|w_{\varepsilon_{n}, j}-w_{0 . j}\right|_{\varepsilon_{n}} \rightarrow 0$ quando $n \rightarrow \infty$. Seja $j \in \mathbb{N}$. Notemos que:

$$
\begin{aligned}
\left|w_{\varepsilon_{n}, j}-w_{0, j}\right|_{\varepsilon_{n}}^{2} & =u_{\varepsilon_{n}}\left(w_{\varepsilon_{n}, j}-w_{0, j}, w_{\varepsilon_{n}, j}-w_{0, j}\right)+\left|w_{\varepsilon_{n}, j}-w_{0, j}\right|_{L^{2}(\Omega 2)}^{2} \\
& =a_{\varepsilon_{n}}\left(w_{\varepsilon_{n}, j}, w_{\varepsilon_{n}, j}\right)-2 a_{\varepsilon_{n}}\left(w_{\xi_{n}, j}, w_{0, j}\right)+a_{0}\left(w_{0, j}, w_{n_{j}, j}\right)+\left|w_{\varepsilon_{n}, j}-w_{0, j}\right|_{L^{2}(\Omega)}^{2} \\
& =\lambda_{\varepsilon_{n}, j}-2 a_{\varepsilon_{n}}\left(w_{\varepsilon_{n}, j}, w_{0, j}\right)+\mu_{j}\left\langle w_{0, j}, w_{0, j}\right\rangle+\left|w_{\varepsilon_{n}, j}-w_{0, j}\right|_{L^{2}(\Omega)}^{2} \\
& =\lambda_{\varepsilon_{n}, j}-2 \int_{\Omega} \nabla_{x} w_{\varepsilon_{n}, j} \cdot \nabla_{x} w_{0, j} \mathrm{~d} x \mathrm{~d} y+\mu_{\jmath}+\left|w_{\varepsilon_{n}, j}-w_{0, j}\right|_{L^{2}(\Omega)}^{2}
\end{aligned}
$$

Como $w_{\varepsilon_{h}, j} \rightarrow w_{0, j}$ em $H^{1}(\Omega)$, temos que

$$
\int_{\Omega}^{n} \nabla_{x} w_{\varepsilon_{n}, j} \cdot \nabla_{x} w_{0, j} \mathrm{~d} x \mathrm{~d} y \rightarrow \int_{j !} \nabla_{x} w_{0, j} \cdot \nabla_{x} w_{0, j} \mathrm{~d} x \mathrm{~d} y=\mu_{i} \text { quando } n \rightarrow \infty \text {. }
$$

O fato de que $w_{\varepsilon_{n}, j} \rightarrow w_{n_{j}}$ em $L^{2}(\Omega)$ para cada $j \in \mathbb{N}$, juntamente com (3.3) e (3.4) implicam que

$$
\lim _{n \rightarrow \infty}\left|w_{s_{n, j}}-w_{0, j}\right|_{\varepsilon_{n}}^{2}=0 .
$$

Aplicando a Proposição 2.3 .5 obtemos que $w_{\varepsilon_{n}, j} \rightarrow w_{0, j}$ em $H I^{1}(\Omega)$ e $a_{\varepsilon_{n}}\left(w_{\varepsilon_{n}, j}, w_{\varepsilon_{n}, j}\right) \rightarrow$ $a_{0}\left(w_{0, j}, w_{0, j}\right)$ quando $n \rightarrow \infty$.

Por meio do Processo Diagonal de Cantor (cf. [26], Teorema 3.144), podemos encontrar uma subsequência de $\left(\varepsilon_{n}\right)_{n \in \mathbb{N}}$, novamente denotada por $\left(\varepsilon_{n}\right)_{n \in \mathbb{N}}$, e uma sequência de funções $\left(u_{0, j}\right)_{j \in \mathbb{N}}$ em $i_{i}^{1}(\bar{l})$ i) satisfazcndo as seguintes propriedades para cada $j \in \mathbb{N}$ :

(1) $\left(\mu_{j}, w_{0, j}\right)$ é um par autovalor-autovetor de $\left(a_{0}, b_{0}\right)$;

(2) $\left|w_{0, j}\right|_{L^{2}(\Omega)}=1$;

(3) $\left|w_{\varepsilon_{n}, j}-w_{0, j}\right|_{\varepsilon_{n}} \rightarrow 0$ quando $n \rightarrow \infty$.

Mostremos que $\left(w_{0 . j}\right)_{j \in \mathbb{N}}$ é um sistema $L_{s}^{2}(\Omega)$-ortonormal. Como $\left|w_{0, j}\right|_{I^{2}(\Omega)}=1$ para todo $j \in \mathbb{N}$, basta mostrarmos que $w_{0, j_{1}}$ e $w_{0, j_{2}}$ são ortogonais pará $j_{1} \neq j_{2}$. Sejam $j_{1}, j_{2} \in \mathbb{N}$ tais que $j_{1} \neq j_{2}$. Então

$$
\int_{, / \Omega} w_{\varepsilon_{n} \cdot j_{1}} w_{\varepsilon_{n} j_{2}} \mathrm{~d} x \mathrm{~d} y=0 \text {, para todo } n \in \mathbb{N} \text {. }
$$

Segue que, para todo $n \in \mathbb{N}$,

$$
\begin{aligned}
\int_{\Omega} w_{0, j_{1}} w_{0, j_{2}} \mathrm{~d} x \mathrm{~d} y & =\int_{\Omega} w_{0, j_{1}} w_{0, j_{2}} \mathrm{~d} x \mathrm{~d} y+\int_{\Omega \Omega}^{r} w_{\varepsilon_{n}, j_{1}} w_{\varepsilon_{n}, j_{2}} \mathrm{~d} x \mathrm{~d} y \\
& -\int_{J_{\Omega}} w_{\varepsilon_{n}, j_{1}} w_{0, j_{2}} \mathrm{~d} x \mathrm{~d} y+\int_{\Omega \Omega} w_{\varepsilon_{n}, j_{1}} w_{0, j_{2}} \mathrm{~d} x \mathrm{~d} y \\
& =\int_{\Delta \downarrow}\left(w_{0, j_{1}}-w_{\varepsilon_{n}, j_{1}}\right) w_{0, j_{2}} \mathrm{~d} x \mathrm{~d} y+\int_{\Omega}\left(w_{\varepsilon_{n}, j_{2}}-w_{0, j_{2}}\right) w_{\varepsilon_{n}, j_{1}} \mathrm{~d} x \mathrm{~d} y
\end{aligned}
$$


Como $w_{\varepsilon_{n}, j} \rightarrow w_{0, j}$ em $L^{2}(\Omega)$, a desigualdade de Hölder implica que

$$
\int_{\Omega} w_{0, j_{1}} w_{0, j_{2}} \mathrm{~d} x \mathrm{~d} y=0
$$

Portanto $w_{0, j_{1}}$ e $w_{0, j_{2}}$ são ortogonais.

Para verificarmos que $\lambda_{0,3}=\mu_{\jmath}$ para todo $j \in \mathbb{N}$, utilizaremos a Proposição 1.1.5. Assim temos somente que mostrar que, para cada $j \in \mathbb{N}$,

$$
\begin{aligned}
\mu_{j} & =a_{0}\left(w_{0, j}, w_{0, j}\right) \\
& =\min \left\{\left.a_{0}(w, w)\left|w \in H_{s}^{1}(\Omega),\right| w\right|_{L_{s}^{2}(\Omega)}=1,\left\langle w, w_{0, h}\right\rangle=0, \text { para } h=1, \ldots, j-1\right\} .
\end{aligned}
$$

Fixemos então $j \in \mathbb{N}$ e seja $w \in H_{w}^{i}\left(\Omega \zeta j\right.$ arbitrário tal que $|w|_{L^{2}(\Omega)}=1$ e $\left\langle w, w_{0, h}\right\rangle=0$ $h=1, \ldots, j-1$. Para cada $n \in \mathbb{N}$, definimos

$$
\left.v_{n}:=-\sum_{h=1}^{j-1} i w, w_{\varepsilon_{n}, h}\right\rangle w_{\varepsilon_{n}, h}+w
$$

Segue que, para cada $n \in \mathbb{N}$ e $k=1, \ldots, j-1$,

$$
\left\langle v_{n}, w_{\varepsilon_{n}, k}\right\rangle=\left\langle w, w_{\varepsilon_{n}, k}\right\rangle-\sum_{h=1}^{j-1}\left\langle w, w_{\varepsilon_{n}, h}\right\rangle\left\langle w_{\varepsilon_{n}, k}, w_{\varepsilon_{n}, h}\right\rangle=0 .
$$

Afirmamos que $\left|v_{n}-w\right|_{\varepsilon_{n}} \rightarrow 0$ quando $n \rightarrow \infty$. De fato, para cada $n \in \mathbb{N}$,

$$
\begin{aligned}
\left|v_{n}-w\right|_{\varepsilon_{n}}^{2} & =\left|\sum_{h=1}^{j-1}\left\langle w, w_{\varepsilon_{n}, h}\right\rangle w_{\varepsilon_{n}, h}\right|_{\varepsilon_{n}}^{2} \\
& =\sum_{h=1}^{j-1}\left\langle u,, w_{\varepsilon_{n}, h}\right\rangle a_{\varepsilon_{n}}\left(w_{\varepsilon_{n}, h}, \sum_{h=1}^{j-1}\left\langle u, u u_{\varepsilon_{n}, h}\right\rangle w_{\varepsilon_{n}, h}\right)+\left|\sum_{h=1}^{j-1}\left\langle w, w_{\varepsilon_{n}, h}\right\rangle w_{\varepsilon_{n}, h}\right|_{L^{2}(\Omega)}^{2} .
\end{aligned}
$$

Utilizando a Identidade de Parseval temos

$$
\begin{aligned}
\left|v_{n}-w\right|_{\varepsilon_{n}}^{2} & =\sum_{h=1}^{j-1} \sum_{k=1}^{j-1}\left\langle w, w_{\varepsilon_{n}, k}\right\rangle\left\langle w, w_{\varepsilon_{n}, h}\right\rangle a_{\varepsilon_{n}}\left(w_{\varepsilon_{n}, h}, w_{\varepsilon_{n}, k}\right)+\sum_{h=1}^{j-1}\left|\left\langle w, u_{\varepsilon_{n}, h}\right\rangle\right|^{2} \\
& =\sum_{h=1}^{j-1} \sum_{k=1}^{j-1}\left\langle w, w_{\varepsilon_{n}, k}\right\rangle\left\langle w, w_{\varepsilon_{n}, h}\right\rangle \lambda_{\varepsilon_{n}, h}\left\langle w_{\varepsilon_{n}, h}, w_{\varepsilon_{n}, k}\right\rangle+\sum_{h=1}^{j-1}\left|\left\langle w, w_{\varepsilon_{n}, h}\right\rangle\right|^{2} .
\end{aligned}
$$

Como $w_{\varepsilon_{n}, h} \rightarrow w_{0, h}$ em $L^{2}(\Omega)$ quando $n \rightarrow \infty$ e $\left\langle w, w_{0, h}\right\rangle=0$ para cada $h=1, \ldots, j-1$, temos que $\left|v_{n}-w\right|_{\varepsilon_{n}}^{2} \rightarrow 0$ quando $n \rightarrow \infty$.

Segue da Proposição 2.3.5 que $v_{n} \rightarrow w$ em $H^{1}(\Omega)$ e, consequentemente, em $L^{2}(\Omega)$. Portanto, $\left|v_{n}\right|_{L^{2}(\Omega)} \rightarrow 1$ quando $n \rightarrow \infty$. Logo, podemos assumir que $\left|v_{n}\right|_{L^{2}(\Omega)} \neq 0$ para todo $n \in \mathbb{N}$. Definimos então

$$
u_{n}:=\left|v_{n}\right|_{L^{2}(\Omega)}^{-1} v_{n}
$$


Temos que $\left|w_{n}\right|_{L^{2}(\Omega)}=1 \mathrm{e}\left\langle w_{n}, w_{\varepsilon_{n}, h}\right\rangle=0, h=1 \ldots, j-1$. Além disso,

$$
\begin{aligned}
\left|w_{n}-w\right|_{\varepsilon_{n}} & =\left.|| v_{n}\right|_{L^{2}(\Omega)} ^{-1} v_{n}-\left.w\right|_{\varepsilon_{n}}=\left.\left.\left|v_{n}\right|_{L^{2}(\Omega)}^{-1}\left|v_{n}-\right| v_{n}\right|_{L^{2}(\Omega)} w\right|_{\varepsilon_{n}} \\
& \leq\left|v_{n}\right|_{L^{2}(\Omega)}^{-1}\left(\left|v_{n}-w\right|_{\varepsilon_{n}}+\left.\left.|w-| v_{n}\right|_{L^{2}(\Omega)} w\right|_{\varepsilon_{n}}\right) \\
& =\left|v_{n}\right|_{L^{2}(\Omega)}^{-1}\left(\left|v_{n}-w\right|_{\varepsilon_{n}}+\left.\left.|1-| v_{n}\right|_{L^{2}(\Omega)}\right|_{\varepsilon_{n}}|w|_{\varepsilon_{n}}\right) .
\end{aligned}
$$

Notemos que $|w|_{\varepsilon_{n}}=|w|_{H^{1}(\Omega)}$, já que $w \in H_{s}^{1}(\Omega)$ e recordemos que $\left|v_{n}\right|_{L^{2}(\Omega)} \rightarrow 1 \mathrm{e}$ $\left|v_{n}-w\right|_{\varepsilon_{n}} \rightarrow 0$ quando $n \rightarrow \infty$. Logo

$$
\left|w_{n}-w\right|_{\varepsilon_{n}} \rightarrow 0 \text { quando } n \rightarrow \infty .
$$

Pela Proposição 2.3.5, sabemos que $a_{\varepsilon_{n}}\left(w_{n}, w_{n}\right) \rightarrow a_{0}(w, w)$ quando $n \rightarrow \infty$. Por outro lado, para cada $j \in \mathbb{N}$,

$$
a_{\varepsilon_{n}}\left(w_{\varepsilon_{n}, j}, w_{\varepsilon_{n}, j}\right)=\dot{\lambda}_{\varepsilon_{n}, j} \rightarrow \mu_{j}=a_{0}\left(w_{0, j}, w_{0, j}\right) \text { quando } n \rightarrow \infty .
$$

Além disso, como $\left(w_{\varepsilon_{n}, j}\right)_{j \in \mathbb{N}}$ é um sistema ortonormal de autovetores do par $\left(a_{\varepsilon_{n}}, b_{0}\right)$, temos que, para todo $n \in \mathbb{N}$ e todo $j \in \mathbb{N}$,

$$
a_{\varepsilon_{n}}\left(w_{\varepsilon_{n}, j}, w_{\varepsilon_{n}, j}\right) \leq a_{\varepsilon_{n}}\left(w_{n}, w_{n}\right) .
$$

Fazendo $n \rightarrow \infty$, segue de (3.5) e (3.6) que

$$
a_{0}\left(w_{0, j}, w_{0, j}\right) \leq a_{0}(w, w) \text { para cada } \jmath \in \mathbb{N} .
$$

Mostramos que, para cada $j \in \mathbb{N}$,

$a_{0}\left(w_{0, j}, w_{0, j}\right)=\min \left\{\left.a_{0}(w, w)\left|w \in H_{s}^{\mathrm{i}}(\Omega),\right| w\right|_{L_{s}^{2}(\Omega)}-1,\left\langle w, w_{0, h}\right\rangle=0, h=1, \ldots, j-1\right\}$, concluindo assim a demonstração clo teorema.

\subsection{Monotonicidade}

O Teorema 3.1.1 implica que, para cada $j \in \mathbb{N}$, a família de autovalores $\left(\lambda_{\varepsilon, 3}\right)_{\varepsilon>0}$ é monótona decrescente e converge para $\lambda_{0, j}$. Observemos que $\lambda_{\varepsilon, 1}=0$ para todo $\varepsilon \geq 0$. Por outro lado, é mostrado em [4] que, se $j \geq 2$, sob condições adicionais, a família $\left(\lambda_{\varepsilon, j}\right)_{\varepsilon>0}$ é estritamente monótona decrescente. Descreveremos aqui tal resultado. Iniciamos apresentando uma condição necessária e suficiente para que a monotonicidade seja estrita. Usarcmos a scguinte notação:

$$
\langle\nabla w, \nabla v\rangle=\int_{\Omega} \nabla w \cdot \nabla v \mathrm{~d} x \mathrm{~d} y, \text { sempre que } w, v \in H^{1}(\Omega) .
$$


Teorema 3.2.1 Sejam $\varepsilon>0$. Consideremos $\left(\lambda_{\varepsilon, j}\right)_{j \in \mathbb{N}}$ a familia de autovalores do par $\left(a_{\varepsilon}, b\right)$.

(1) Suponhamos que exista um $j \in \mathbb{N}$ e números reais $0<\varepsilon_{2}<\varepsilon_{1}$ tais que $\lambda_{\varepsilon_{2}, j}=\lambda_{\varepsilon_{1}, j}$. Entĩo existe uma função $w \in H_{s}^{i}(s i)$ com $w \neq 0$, tal que

$$
\langle\nabla w, \nabla v\rangle=\lambda\langle w, v\rangle \text { qualquer que seja } v \in H^{1}(\Omega),
$$

onde $\lambda=\lambda_{\varepsilon_{2, j}, j}$;

(2) reciprocamente, se existe $u m \lambda \in \mathbb{R}$ e um $w \in H_{s}^{1}(\Omega)$, com $w \neq 0$, tais que (3.7) esteja satisfeita, então existe um único $k \in \mathbb{N}$ com $\lambda=\lambda_{n_{1}:}<\lambda_{0, k+1}$. Além disso, existe um $\varepsilon_{0}>0$ tal que

$$
\lambda_{\varepsilon, k}=\lambda \text { para todo } \varepsilon \in\left[0, \varepsilon_{0}\right] .
$$

Demonstração. Suponhamos que as hipóteses em (1) estejam satisfeitas. Seja $E$ o subespaço $j$-dimonsional de $H^{1}(\Omega)$ gerado pelo conjunto $\left\{w_{\varepsilon_{2}, 1}, \ldots, w_{\xi_{2}, j}\right\}$. Pela Proposição 1.1.3, temos que

$$
\lambda_{* \cdot, i} \leq \max _{u \in E \backslash\{0\}} \frac{a_{\varepsilon_{1}}(u, u)}{\langle u, u\rangle} .
$$

Consequentemente, existe uma função $w \in E, \operatorname{com}\langle w, w\rangle=1$, tal que

$$
\lambda_{\varepsilon_{1}, j} \leq a_{\varepsilon_{1}}(w, w) .
$$

Nosso primeiro passo será mostrar que $w \in H_{s}^{1}(\Omega)$, isto é, $\nabla_{y} w \ddot{-} 0$ em $L^{2}(\Omega)$. Notemos que

$$
\begin{aligned}
a_{\varepsilon_{1}}(w, w) & =\int_{\Omega}\left(\nabla_{x} w \cdot \nabla_{x} w+\frac{1}{\varepsilon_{1}^{2}} \nabla_{y} w \cdot \nabla_{y} w\right) \mathrm{d} x \mathrm{~d} y \\
& +\frac{1}{\varepsilon_{2}^{2}} \int_{\Omega} \nabla_{y} w \cdot \nabla_{y} w \mathrm{~d} x \mathrm{~d} y-\frac{1}{\varepsilon_{2}^{2}} \int_{j \Omega} \nabla_{y} w \cdot \nabla_{y} w \mathrm{~d} x \mathrm{~d} y \\
& =a_{\varepsilon_{2}}(w, w)-\left(\frac{1}{\varepsilon_{2}^{2}}-\frac{1}{\varepsilon_{1}^{2}}\right)\left\langle\nabla_{y} w, \nabla_{y} w\right\rangle .
\end{aligned}
$$

E assim,

$$
\lambda_{\varepsilon_{1}, j} \leq a_{\varepsilon_{2}}(w, w)-\left(\frac{1}{\varepsilon_{2}^{2}}-\frac{1}{\varepsilon_{1}^{2}}\right)\left\langle\nabla_{y} w, \nabla_{y} w\right\rangle
$$

Como para cada $k=1, \ldots, j, w_{\varepsilon_{2}, k}$ é um autovetor associado ao autovalor $\lambda_{\varepsilon_{2}, k},\left(w_{\varepsilon_{2}, j}\right)_{j \in \mathbb{N}}$ é um sistema $L^{2}(\Omega)$-ortonromal completo e $w \in E$, segue que

$$
\begin{aligned}
a_{\varepsilon_{2}}(w, w) & =\sum_{k=1}^{j} a_{\varepsilon_{2}}\left(w,\left\langle w, w_{\varepsilon_{2}, k}\right\rangle w_{\varepsilon_{2}, k}\right) \\
& =\sum_{k=1}^{j}\left\langle w, w_{\varepsilon_{2}, k}\right\rangle a_{\varepsilon_{2}}\left(w, w_{\varepsilon_{2}, k}\right)=\sum_{k=1}^{j} \lambda_{\varepsilon_{2}, k}\left|\left\langle w, w_{\varepsilon_{2}, k}\right\rangle\right|^{2} .
\end{aligned}
$$


Por outro lado,

$$
\lambda_{\varepsilon_{2}, j}=\lambda_{\varepsilon_{2}, j}\langle w, w\rangle=\lambda_{\varepsilon_{2}, j} \sum_{k=1}^{j}\left|\left\langle w, w_{\varepsilon_{2}, k}\right\rangle\right|^{2}
$$

Como estamos supondo $\lambda_{\varepsilon_{2} . j}=\lambda_{\varepsilon_{1}, j}$, a desigualdade (3.8) implica que

$$
\begin{aligned}
\lambda_{\varepsilon_{2, j} j} \sum_{k=1}^{j}\left|\left\langle w, w_{\varepsilon_{2}, k}\right\rangle\right|^{2} & =\lambda_{\varepsilon_{2, j}}=\lambda_{\varepsilon_{1} j} \leq a_{\varepsilon_{2}}(w, w)-\left(\frac{1}{\varepsilon_{2}^{2}}-\frac{1}{\varepsilon_{1}^{2}}\right)\left\langle\nabla_{y} u, \nabla_{y} w\right\rangle \\
& =\sum_{k=1}^{j} \lambda_{\varepsilon_{2}, k}\left|\left\langle w, w_{\varepsilon_{2}, k}\right\rangle\right|^{2}-\left(\frac{1}{\varepsilon_{2}^{2}}-\frac{1}{\varepsilon_{1}^{2}}\right)\left\langle\nabla_{y} w, \nabla_{y} w\right\rangle,
\end{aligned}
$$

ou seja,

$$
\sum_{k=1}^{j}\left(\lambda_{\varepsilon_{2}, j}-\lambda_{\varepsilon_{2}, \cdots}\right) \mid\left\langle\left.\left(w, w_{z_{\alpha}, \ldots}\right)\right|^{\hat{z}} \leq-\left(\frac{1}{\varepsilon_{2}^{2}}-\frac{1}{\varepsilon_{1}^{2}}\right)\left\langle\nabla_{y} w, \nabla_{y} w\right\rangle .\right.
$$

Notemos que $\lambda_{\varepsilon_{2, j}} \geq \lambda_{\varepsilon_{2}} k$ para cada $k=1, \ldots, j$, e que

$$
-\left(\frac{1}{\varepsilon_{2}^{2}}-\frac{1}{\varepsilon_{1}^{2}}\right)<0 \text {. }
$$

Portanto,

$$
\sum_{k-1}^{i}\left(\lambda_{s_{2}, j}-\lambda_{\varepsilon_{2}, k}\right)\left|\left\langle w, w_{\varepsilon_{2}, k}\right\rangle\right|^{2} \geq 0
$$

$$
-\left(\frac{1}{\varepsilon_{2}^{7}}-\frac{1}{\varepsilon_{1}^{2}}\right)\left\langle\nabla_{y} w, \nabla_{y} w\right\rangle \leq 0 .
$$

Esses fatos e a desigualdade (3.9) implicam que devemos ter

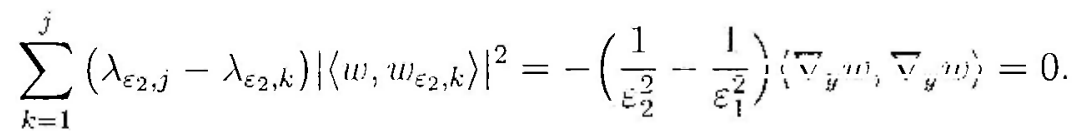

Concluímos que

$$
\left\langle\nabla_{y} w, \nabla_{y} w\right\rangle=0
$$

ou seja, $w \in H_{s}^{1}(\Omega)$. Notemos ainda que por $(3.10)$

$$
\left|\left\langle w, w_{\varepsilon_{2}, k}\right\rangle\right|^{2}=0 \text { semprè que } \lambda_{\varepsilon_{2}, j}-\lambda_{\varepsilon_{2}, k}>0
$$

Ou seja, $w \in E$ e $w$ é ortogonal a todos os autovetores associados a $\lambda_{\varepsilon_{2}, k}$, desde que $\lambda_{\Sigma_{2}, k} \not \leq \lambda_{\varepsilon_{2}, j}$. Com isso, $w$ pertence ao autocspaço gerado por $\lambda=\lambda_{\varepsilon_{2}, j}$. Em outras palavras, qualquer que seja $v \in H^{1}(\Omega)$,

$$
\langle\nabla w, \nabla v\rangle=\left\langle\nabla_{x} w, \nabla_{x} v\right\rangle=\left\langle\nabla_{x} w, \nabla_{x} v\right\rangle+\frac{1}{\varepsilon_{2}^{2}}\left\langle\nabla_{y} w, \nabla_{y} v\right\rangle=a_{\varepsilon_{2}}(u, v)=\lambda\langle u, v\rangle .
$$


A demonstração de (1) está concluída.

Demonstremos a parte (2) do teorema. Seja $w \in H_{s}^{1}(\Omega)$ com $w \neq 0$ e $\lambda \in \mathbb{R}$ tais que (3.7) esteja satisfeita. Segue que, para todo $\varepsilon>0$,

$$
a_{\varepsilon}(u, v)-\langle\nabla w, \nabla v\rangle=\lambda\langle w, v\rangle, \text { qualquer que seja } v \in H^{1}(\Omega) .
$$

Assim, para todo $\varepsilon>0$, temos que $\lambda$ é um autovalor do operador $A_{\varepsilon}$. Como $w \in$ $H_{s}^{1}(\Omega)$, segue que

$$
a_{0}(w, v)=\langle\nabla w, \nabla v\rangle-\lambda\langle w, v\rangle
$$

Logo, $\lambda$ é também autovalor de $A_{0}$. Seja $r \in \mathbb{N}$ o maior númcro tal quc $\lambda=\lambda_{0, r}$. Então $\lambda_{0, r}<\lambda_{0, r+1}$. O Teorema 3.1.1 implica que $\lambda_{\varepsilon_{1}, r+1} \geq \lambda_{\varepsilon_{2}, r+1}$ sempre que $0<\varepsilon_{1}<\varepsilon_{2}$. Além disso, segue desse mesmo resultado que $\lambda_{0, r+1}=\sup _{s>0} \lambda_{\varepsilon_{r+1}+1}$, ou seja, $\lambda_{0, r+1} \geq \lambda_{\varepsilon, r+1}$, para todo $\varepsilon \geq 0$. Portanto, existe um $\varepsilon_{0}>0$ tal que $\lambda_{0, r+1} \geq \lambda_{\varepsilon, r+1}>\lambda_{0, r}=\lambda$ para todo $\varepsilon \in\left[0, \varepsilon_{0}\right]$.

Para complctar a demonstração, basta mostrar que, para todo $\varepsilon \in\left[0, \varepsilon_{0}\right], \lambda_{\varepsilon, r}=\lambda$. Suponhamos que isso não seja rerdadeiro. Então existiria um $\varepsilon \in\left[0, \varepsilon_{0}\right]$ tal que

$$
\lambda_{\bar{\varepsilon}, r}<\lambda<\lambda_{\bar{c}, r+1}
$$

mas isso contradiz o fato de $\lambda$ ser um autovalor de $A_{\bar{\xi}}$.

$O$ próximo resultado apresenta as condliçoes quo garantem a monotonicidade estrita da família $\left(\lambda_{\varepsilon, j}\right)_{\varepsilon>0}$. para cada $j \geq 2$.

Teorema 3.2.2 Suponhamos que exista um conjunto aberto nâo-vazio $J \subset \mathbb{R}^{M}$ e dois conjuntos abertos e disjuntos $U, U^{\prime} \subset \mathbb{R}^{M+N}$ lais que $\Omega \cap\left(J \times \mathbb{R}^{N}\right)=U \cup U^{\prime}$ e que para todo $x \in J$, a seção vertical

$$
U_{x}:=\{y \mid(x, y) \in U\}
$$

seja conexa e não-vazia. Para $x \in J$, seja $p(x)>0$ a medida de Lebesgue $N$-dimensional da seçâo vertical $U_{x}$. Suponhamos ainda que $p \in C^{1}(J)$ e que $\nabla p \neq 0$ em J. Então, para todo $j \in \mathbb{N}$ com $j \geq 2$, a família $\left(\lambda_{\varepsilon, j}\right)_{\varepsilon>0}$ é estritamente monótona decrescente.

Demonstraçāo. Suponhamos que as conclusões do teorema não sejam verdadeiras. Segue do Teorema 3.2 .2 que existe um número real $\lambda \neq 0$ e $w \in H_{s}^{1}(\Omega)$ com $w \neq 0$, tais que

$$
\langle\nabla w, \nabla v\rangle=\lambda\langle w, v\rangle \text { qualquer que seja } v \in H^{1}(\Omega) .
$$

Afirmamos que $w$ é uma função analítica real. De fato, qualquer que seja $\varphi \in \mathcal{D}(\Omega)$, o Teorema 1.4 .2 de [23] implica que

$$
\int_{\Omega} w \Delta \varphi \mathrm{d} x \mathrm{~d} y=\int_{\partial \Omega}(\nabla w \cdot \nabla \varphi) \nu \mathrm{d} \sigma-\int_{\Omega} \nabla w \cdot \nabla \varphi \mathrm{d} x \mathrm{~d} y
$$


onde $\sigma$ é a medida de Hausdorff e $\nu(x, y)$ é a componente $x$ da normal exterior a $\partial \Omega$ em $(x, y) \in \partial \Omega$. Como $\varphi \in \mathcal{D}(\Omega)$, temos que

$$
\int_{\partial \Omega}(\nabla w \cdot \nabla \varphi) \nu \mathrm{d} \sigma=0
$$

Sendo assim,

$$
\int_{\delta \Omega} w \Delta \varphi \mathrm{d} x \mathrm{~d} y=-\int_{\Omega} \nabla w \cdot \nabla \varphi \mathrm{d} x \mathrm{~d} y=-\lambda \int_{\Omega \Omega} w \varphi \mathrm{d} x \mathrm{~d} y .
$$

Logo, $\Delta w=-\lambda w$. Agora, os resultados das Seções 2.1 e 4.2 do Capítulo V de $[7]$ implicam que uma solução de $(\Delta+\lambda) u=0$ é uma função analítica. Assim, $w$ é uma função analítica. Como $w \in I_{s}^{1}(\Omega)$, temos que $\nabla_{y} w=0$ cm $\Omega$. Como $U_{x}$ é conexo para todo $x \in J$, segue que existe uma função $\tilde{w}: J \rightarrow \mathbb{R}$ com $\tilde{w}(x)=w(x, y)$ sempre que $(x, y) \in U$. Isto implica que $\tilde{w} e ́$ uma função analítca real em $J$ e

$$
\Delta_{x} \tilde{w}=-\lambda \tilde{w} \text { em } J
$$

Agora, dada uma função $\bar{v} \in \mathcal{D}(J)$, definimos $v: \Omega \rightarrow \mathbb{R}$ por:

$$
v(x, y):= \begin{cases}\tilde{v}(x), & \text { se }(x, y) \in U \\ 0, & \text { caso contrário. }\end{cases}
$$

Com isso $v \in C^{\infty}(\Omega), \nabla_{y} v=0 \mathrm{e}$

$$
\nabla_{x} v(x, y):= \begin{cases}\nabla \tilde{v}(x), & \text { se }(x, y) \in U \\ 0, & \text { caso contrário. }\end{cases}
$$

Assim tanto $v$ quanto $\nabla v$ são funções limitadas em $\Omega$ e, portanto, $v \in H^{1}(\Omega)$. Logo,

$$
\begin{aligned}
\langle\nabla w, \nabla v\rangle & =\int_{U} \nabla w \cdot \nabla v \mathrm{~d} x \mathrm{~d} y=\int_{J} p(x) \nabla \tilde{w}(x) \cdot \nabla \tilde{v}(x) \mathrm{d} x \\
& =\int_{J} p\left(\sum_{i=1}^{M} \partial_{x_{i}} \tilde{w} \partial_{x_{i}} \tilde{v}\right) \mathrm{d} x=\sum_{i=1}^{M} \int_{J}\left(p \partial_{x_{i}} \tilde{w}\right) \partial \tilde{v} \mathrm{~d} x \\
& =-\sum_{i=1}^{M} \int_{J} \partial_{x_{i}}\left(p \partial_{x_{i}} \tilde{w}\right) \tilde{v} \mathrm{~d} x=-\sum_{i=1}^{M} \int_{J}\left(\partial_{x_{i}} p \partial_{x_{i}} \tilde{w}+p \partial_{x_{i}}^{2} \tilde{w}\right) \tilde{v} \mathrm{~d} x \\
& =-\int_{J}(\nabla p \cdot \nabla \tilde{w}) \tilde{v} \mathrm{~d} x-\int_{J} p \tilde{v} \Delta \tilde{w} \mathrm{~d} x
\end{aligned}
$$

Como

$$
\int_{J} p \tilde{v} \Delta \tilde{w} \mathrm{~d} x=-\lambda \int_{J} p \tilde{v} \tilde{w} \mathrm{~d} x
$$

segue que

$$
\langle\nabla w, \nabla v\rangle=\lambda \int_{J} p \tilde{v} \tilde{w} \mathrm{~d} x-\int_{. J}(\nabla p \cdot \nabla \tilde{w}) \tilde{v} \mathrm{~d} x
$$


Por outro lado, (3.11) implica que

$$
\langle\nabla w, \nabla v\rangle=\lambda\langle w, v\rangle=\lambda \int_{I I}^{r} w v \mathrm{~d} x=\lambda \int_{I}^{r} p \tilde{w} \tilde{w} \mathrm{~d} x
$$

Segue de (3.13) e (3.14) que

$$
\int_{J}(\nabla p \cdot \nabla \tilde{u}) \tilde{r} \mathrm{~d} x=0 \text { qualquer que seja } \hat{v} \in \mathcal{D}(J) \text {. }
$$

Portanto, $\nabla p \cdot \nabla \tilde{w}=0$ em $J$. Como $\nabla p$ não é identicamente nulo em $J$, segue que existe um aberto conexo $J^{\prime}$ de $J$ tal que

$$
\nabla \tilde{w}=0 \mathrm{em} J^{\prime}
$$

Como $\lambda \neq 0$, a equaşão (3.12) implica que $\tilde{w}=0 \mathrm{em} J^{\prime}$. Portanto $w(x, y)=0$ para todo $(x, y) \in \Omega^{\prime}:=U \cap\left(J^{\prime} \times \mathbb{R}^{N}\right)$. É claro que $\Omega^{\prime}$ é aberto.

Como $J^{\prime}$ é não-vazio e $U_{x}$ é não-vazio para todo $x \in J$, segue que $\Omega^{\prime}$ e também não-vazio. Como $w$ é uma função analítica, scgue que $w$ é identicamente nula, o que ó uma contraclição.

Seja $P: \mathbb{R}^{M+N} \longrightarrow \mathbb{R}^{M}$ dada por $P(x, y):=x$. O Teorema 3.2 .2 nos diz que, se existir um conjunto aberto não-vazio $J \subset P(\Omega)$ tal cuc $\Omega \cap\left(J \times \mathbb{R}^{N}\right)$ ć umião do subconjuntos abertos e disjuntos, e que para pelo menos um desses conjuntos, digamos $U$, a seção vertical $U_{x}$ é não vazia e concxa para todo $x \in J$ e a função $p$ ć não constantc c $p \in C^{1}(J)$, então temos a monotonicidade da família $\left(\lambda_{\varepsilon, 0}\right)_{\varepsilon>0}$ para todo $j \in \mathbb{N} \operatorname{com} j \geq 2$. Os domínios considerados por Hale e Raugel em [14] satisfazem tal condição desde que a função $g$ não seja constante. Já os domínios em forma de $C$, que definiremos no Capítulo 6, não satisfazem tal condição. 


\section{Capítulo 4}

\section{Convergência de Semigrupos e de Semifluxos}

Nesse capítulo analisaremos o comportamento da farmíla de semifluxos $\left(\pi_{\Sigma}\right)_{\varepsilon>0}$ quando $\varepsilon \rightarrow 0^{+}$.

Recordemos que, para cada $\varepsilon>0, \pi_{\varepsilon}$ é o semifluxo gerado pelas soluções da equação de evolução

$$
\dot{u}+A_{\varepsilon} u=\dot{f}(u),
$$

onde $A_{\varepsilon}$ é o operador gerado pclo par $\left(a_{\varepsilon},\langle\cdot,\rangle_{L^{2}(\Omega)}\right)$. Recordemos também que $\pi_{0}$ é o semifluxo gerado pelas soluções da equação de evolução

$$
\dot{u}+A_{0} u=\hat{f}(u),
$$

onde $A_{0}$ é o operador gerado pelo par $\left(a_{0},\langle\cdot\rangle_{L^{2}(\Omega)}\right)$.

Nossa análise inicia-se com o problema linear associado às equações $\left(R D_{\varepsilon}\right), \varepsilon>0$, e $\left(R D_{0}\right)$. Esse estudo é descrito na próxima seção e na Seção 4.2 analisaremos a família $\left(\pi_{\varepsilon}\right)_{\varepsilon>0}$.

Em todo esse capítulo, utilizaremos a notação apresentada nos Capítulos 2 e 3.

\subsection{Convergência de semigrupos}

Recordemos que o semigrupo gerado pelo operador $-A_{\hat{\varepsilon}}, \varepsilon>0$, é denotado por $e^{-A_{\varepsilon} t}$, $t \geq 0$.

Para o nosso primeiro resultado de convergência de semigrupos serão necessários alguns resultados preliminares.

Lema 4.1.1 Dados $\beta \in(0, \infty)$ e $\delta>0$, existe um $s_{0}=s_{0}(\delta, \beta) \in \mathbb{R}$ tal que $(s+1) e^{-s t}<\delta$ para todo $s \geq s_{0}$ e todo $t \geq \beta$. 
Demonstração. Fixemos $\beta>0$ e seja $t \geq \beta$. Então,

$$
(s+1) e^{-s t} \leq(s+1) e^{-\ldots f} \text { para todo } s \geq 0 \text { c para } t \geq \beta .
$$

Sabemos que $\lim _{s \rightarrow \infty}(s+1) e^{-s \beta}=0$. Portanto, dado $\delta>0$, existe um $s_{0}=s_{0}(\delta, \beta)>0$ tal que

$$
(s+1) e^{-s \beta}<\delta \text { para todo } s \geq s_{0} .
$$

Utilizando (4.1) e (4.2) temos que $(s+1) e^{-s t} \leq \delta$ para todo $s \geq s_{0}$ e todo $t \geq \beta$.

Lema 4.1.2 Fixemos $j \in \mathbb{N}$. Dado $n \in \mathbb{N}$, seja $P_{n}: L^{2}(\Omega) \longrightarrow L^{2}(\Omega)$ a projeção $L^{2}(\Omega)$

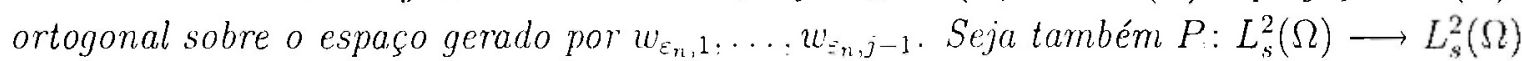
a projeção $L_{s}^{2}(\Omega)$-ortogonal de $L_{s}^{2}(\Omega)$ sobre o espaço gerado por $w_{0,1}, \ldots \ldots w_{i, j-i}$. Então, para todo $n \in \mathbb{N}$, todo $t>0$ e toda função $u \in L^{2}(\Omega)$. temos:

$$
\begin{aligned}
P_{n} e^{-A_{s_{n}} t} u & =e^{-A_{s_{n}} t} P_{n} u \in D\left(A_{\varepsilon_{n}}\right) \subset H^{1}(\Omega), \\
\left(I-P_{n}\right) e^{-A_{s_{n}} t} u & =e^{-A_{s_{n}} t}\left(I-P_{n}\right) u \in D\left(A_{\varepsilon_{n}}\right) \subset I^{1}(\Omega) .
\end{aligned}
$$

Se $u \in L_{s}^{2}(\Omega)$, então

$$
\begin{aligned}
P e^{-A_{0} t} u & =e^{-A_{0} t} P u \in D\left(A_{0}\right) \subset H_{s}^{1}(\Omega) . \\
(I-P) e^{-A_{0} t} u & =e^{-A_{0} t}(I-P) u \in D\left(\Lambda_{0}\right) \subset I I_{s}^{1}(\Omega) .
\end{aligned}
$$

Demonstração. Vamos denotar apenas por $\langle\cdot:\rangle$ o produto interno de $L^{2}(\Omega)$. Se $u \in$ $L^{2}(\Omega)$, como $\left(w_{\varepsilon_{n}, 2}\right)_{\imath \in \mathbb{N}}$ é um sistema $L^{2}(\Omega)$-ortonormal, podemos escrever

$$
u=\sum_{i=1}^{\infty}\left\langle u, w_{\varepsilon_{n}, i}\right\rangle w_{s_{n}, i}
$$

Para todo $n \in \mathbb{N}$ e todo $t \geq 0$, o operador $e^{-A_{\varepsilon_{n}} t}$ é limitado. Portanto

$$
e^{-A_{z_{n}} t} u=\sum_{i=1}^{\infty}\left\langle u, w_{\varepsilon_{n}, i}\right\rangle e^{-A_{\varepsilon_{n}} t} w_{\varepsilon_{n}, i}
$$

A Proposição 1.1.9 implica que

$$
e^{-A_{\Sigma n} t} u=\sum_{i=1}^{\infty} e^{-t \lambda_{n_{n}, i}}\left\langle u, w_{s_{n},}\right\rangle w_{\varepsilon_{n}, i} .
$$

Logo,

$$
P_{n} e^{-A_{\varepsilon_{n}} t} u=\sum_{i=1}^{\infty} e^{-t \lambda_{s_{n}, i}}\left\langle u, w_{\varepsilon_{n}, i}\right\rangle P_{n} w_{\varepsilon_{n}, i}=\sum_{i=1}^{j-1} e^{-l \lambda_{\varepsilon_{n}, i}}\left\langle u, w_{\varepsilon_{n}, i}\right\rangle w_{\varepsilon_{n}, i} .
$$

Por outro lado,

$$
P_{n} u=\sum_{i=1}^{j-1}\left\langle u, w_{\varepsilon_{n}, i}\right\rangle w_{\varepsilon_{n}, i}
$$


o que implica que

$$
e^{-A_{\varepsilon_{n}} t} P_{n} u=\frac{\sum_{i=1}^{j-1}}{e^{-i \dot{A}_{n, i}}}\left\langle u, w_{\varepsilon_{n}, i}\right\rangle w_{\varepsilon_{n}, i} .
$$

As igualdades (4.5) e (4.6) implicam a primeira igualdade em (4.3).

Agora notemos que, como $w_{\varepsilon_{n}, j} \dot{\mathrm{e}}$ um autovetor associado a $\lambda_{\varepsilon_{n}, j}$ para cada $n \in \mathbb{N}$, qualquer que seja $v \in H^{1}(\Omega)$ temos que,

$$
\begin{aligned}
& a_{\varepsilon_{n}}\left(\sum_{i=1}^{j-1} e^{-t \lambda_{\varepsilon_{n}, i}}\left\langle u, w_{\varepsilon_{n}, i}\right\rangle w_{\varepsilon_{n}, i}, v\right)=\sum_{i=1}^{j-1} e^{-t \lambda_{\varepsilon_{n}, i}}\left\langle u, w_{\varepsilon_{n}, i}\right\rangle a_{\varepsilon_{n}}\left(w_{\varepsilon_{n}, 2}, v\right) \\
& =\sum_{i=1}^{j-1} e^{-i \lambda_{\varepsilon_{n}, i}} \lambda_{\varepsilon_{n}, i}\left\langle u, w_{\varepsilon_{n}, i}\right\rangle\left\langle w_{\varepsilon_{n}, i}, v\right\rangle \\
& -\left\langle\sum_{i=1}^{j-1} e^{-i \lambda_{z, i}, i} \lambda_{\varepsilon_{n}, i}\left\langle u_{,}, w_{z_{n}, i}\right\rangle u_{z_{n}, i}, v\right\rangle=\left\langle e^{-A_{E_{n}} t} P_{n} \imath t, v\right\rangle .
\end{aligned}
$$

Assim, $P_{n} e^{-A_{\varepsilon_{n}} t} u=e^{-A_{\varepsilon_{n}} t} P_{n} u \in D\left(A_{\tilde{\Sigma}_{n}}\right) \subset I^{1}(\Omega)$ para toda função $u \in L^{2}(\Omega)$.

Analogamente demonstramos a segunda igualdade de (4.3). Para demonstrar (4.4) basta seguirmos os mesmos passos da demonstração de (4.3).

Temos então nosso resultado de convergência dos semigrupos.

Teorema 4.1.3 Seja $\left(\varepsilon_{n}\right)_{n \in \mathbb{N}}$ uma sequência arbitrária de números positivos com $\varepsilon_{n} \rightarrow$ $0^{+}$quando $n \rightarrow \infty$. Suponhamos que $\left(u_{n}\right)_{n \in \mathbb{N}}$ seja uma sequência em $L^{2}(\Omega)$ e que $u \in$ $L_{s}^{2}(\Omega)$ seja tal que $\left|u_{n}-u\right|_{L^{2}(\Omega)} \rightarrow 0$ quando $n \rightarrow \infty$. Então, para todo $\beta \in(0, \infty)$,

$$
\sup _{t \in[\beta, \infty)}\left|e^{-A_{\varepsilon_{n}} t} u_{n}-e^{-A_{0} t} u\right|_{\varepsilon_{n}} \rightarrow 0 \text { quando } n \rightarrow \infty \text {. }
$$

Demonstração. Fixemos $\beta \in(0, \infty)$ e seja $\delta>0$ arbitrário. Pelo Lema 4.1.1 existe um $s_{0}=s_{0}(\delta, \beta)$ tal que $(s+1) e^{-s t}<\delta$ para todo $s \geq s_{0}$ e todo $t \geq \beta$. Existe também um $j_{0}=j_{0}(\delta, \beta)$ tal que $\lambda_{0, j_{0}}>s_{0}$ já que, pela Proposição $1.1 .2, \lambda_{0, j} \rightarrow \infty$, quando $j \rightarrow \infty$. Como pelo Teorema 3.1.1 temos que $\lambda_{0, j_{0}}=\sup _{\varepsilon>0} \lambda_{-; j_{0}}$, segue que existe um $n_{0}=n_{0}(\delta, \beta)$ tal que $\lambda_{\varepsilon_{n}, j_{0}}>s_{0}$ para $n \geq n_{0}$. Mas para cada $\varepsilon \geq 0$, a Proposição 1.1 .2 implica que $\lambda_{\varepsilon, j+1} \geq \lambda_{\varepsilon, j}$ para todo $j \in \mathbb{N}$. Com isso obtemos

$$
\lambda_{\varepsilon_{n, j}} \geq s_{0}(\delta, \beta), \text { para todo } n \geq n_{0} \text { e todo } j \geq j_{0}
$$

Sejam $P_{n}: L^{2}(\Omega) \longrightarrow L^{2}(\Omega)$ a projeção $L^{2}(\Omega)$-ortonormal sobre o espaço gerado por $w_{\varepsilon_{n}, 1}, \ldots, w_{\varepsilon_{n}, \gamma_{0}-1}$ e $P: L_{s}^{2}(\Omega) \longrightarrow L_{s}^{2}(\Omega)$ a projeção $L_{s}^{2}(\Omega)$-ortonormal sobre o espaço gerado por $w_{0,1}, \ldots, w_{1, j_{n-1}}$. Notemos que, para todo $n \in \mathbb{N}$ e todo $t \geq 0$,

$$
\begin{gathered}
\left|e^{-A_{\varepsilon n} t} u_{n}-e^{-A_{0} t} u\right|_{\varepsilon_{n}}=\left|\left(I-P_{n}\right) e^{-A_{\varepsilon_{n}} t} u_{n}-(I-P) e^{-A_{0} t} u+P_{n} e^{-A_{\varepsilon_{n}} t_{n}} u_{n}-P e^{-A_{0} t} u\right|_{\varepsilon_{n}} \\
\leq\left|P_{n} e^{-A_{\varepsilon_{n}} t} u_{n}-P e^{-A_{0} t} u\right|_{\varepsilon_{n}}+\left|\left(I-P_{n}\right) e^{-A_{\varepsilon_{n}} t} u_{n}\right|_{\varepsilon_{n}}+\left|(I-P) e^{-A_{0} t} u\right|_{\varepsilon_{n}} .
\end{gathered}
$$


Vamos analisar cada uma das parcelas da soma acima. Para todo $n \in \mathbb{N}$, o Lema 4.1.2 implica que

$$
\begin{aligned}
\left|P_{n} e^{-A_{\varepsilon_{n}} t} u_{n}-P e^{-A_{0} t} u\right|_{\varepsilon_{n}} & =\left|\sum_{i=1}^{J_{0}-1} e^{-t \lambda_{\varepsilon_{n}, i}}\left\langle u_{n}, w_{\varepsilon_{n}, i}\right\rangle w_{\varepsilon_{n}, i}-\sum_{i=1}^{j_{0}-1} e^{-t \lambda_{0, i}}\left\langle u, w_{0, i}\right\rangle w_{0, i}\right|_{\varepsilon_{n}} \\
& \leq \sum_{i=1}^{j_{0}-1}\left|e^{-t \lambda_{\varepsilon_{n}, i}}\left\langle u_{n}, w_{\varepsilon_{n}, i}\right\rangle w_{\varepsilon_{n, i}, i}-e^{-t \lambda_{0, i}}\left\langle u, w_{0, i}\right\rangle w_{0, i}\right|_{\varepsilon_{n}}
\end{aligned}
$$

Somando e subtraindo a expressão $e^{-t \lambda_{\varepsilon_{n}, i}}\left\langle u_{n}, w_{\varepsilon_{n}, i}\right\rangle w_{0, i}$ obtemos,

$$
\begin{aligned}
& \left|P_{n} e^{-A_{\varepsilon_{n}} t} u_{n}-P e^{-A_{0} t} u\right|_{\varepsilon_{n}} \\
& \leq \sum_{i=1}^{j 0-1}\left|e^{-t \lambda_{\varepsilon_{n}, i}}\left\langle u_{n}, w_{\varepsilon_{n}, i}\right\rangle\left(w_{\varepsilon_{n}, i}-w_{0, i}\right)+\left(e^{-t \lambda_{\varepsilon_{n}, i}}\left\langle u_{n}, w_{\varepsilon_{n}, i}\right\rangle-e^{-t \lambda_{0, i}}\left\langle u, w_{0, i}\right\rangle\right) w_{0, i}\right|_{\varepsilon_{n}} \\
& \leq \sum_{i=1}^{j 0-1}\left(\left|e^{-t \lambda_{\varepsilon_{n} . i}}\left\langle u_{n}, w_{\varepsilon_{n}, i}\right\rangle\right||| w_{\varepsilon_{n}, i}-\left.w_{0, i}\right|_{\varepsilon_{n}}+\left|e^{-t \lambda_{\varepsilon_{n}, i}}\left\langle u_{n}, w_{\varepsilon_{n}, i}\right\rangle-e^{-t \lambda_{0, i}}\left\langle u, w_{0, i}\right\rangle\right|\left|w_{0, i}\right|_{\varepsilon_{n}}\right) .
\end{aligned}
$$

O Teorema 3.1.1 implica que, para cada $i=1, \ldots, j_{0}-1,\left|w_{\varepsilon_{n}, i}-w_{0, i}\right|_{\varepsilon_{n}} \rightarrow 0$ quando $n \rightarrow \infty$. Logo, $\left|w_{\varepsilon_{n}, i}-w_{0, i}\right|_{L^{2}(\Omega)} \rightarrow 0$ quando $n \rightarrow \infty$ para cada $i=1, \ldots, j_{0}-1$. Por hipótese temos que $\left|u_{n}-u\right|_{L^{2}(\Omega)} \rightarrow 0$ quando $n \rightarrow \infty$. Assim, pela continuidade de $\langle\cdot, \cdot\rangle$ em $L^{2}(\Omega)$ segue que $\left\langle u_{n}, w_{\varepsilon_{n}, i}\right\rangle \rightarrow\left\langle u, w_{0, i}\right\rangle$ quando $n \rightarrow \infty$ para $i=1, \ldots, j_{0}-1$.

Afirmamos que, para $i=1, \ldots, j_{0}-1$,

$$
\sup _{t \in[\beta, \infty)}\left|e^{-t \lambda_{\varepsilon_{n}, i}}-e^{-t \lambda_{0.2}}\right| \rightarrow 0, \text { quando } n \rightarrow \infty .
$$

De fato, fixemos $i \in\left\{1, \ldots, j_{0}-1\right\}$ e seja $t \in[\beta, \infty)$. É claro que a afirmação é válida se $\lambda_{0, i}=0$. Suponhamos então que $\lambda_{0, i}>0$. Portanto, existe um $\alpha \in \mathbb{R}$ tal que $0<\alpha<\lambda_{0, i}$. Como $\lambda_{\varepsilon_{n}, l} \rightarrow \lambda_{0, i}$, segue que existe um $n_{1} \in \mathbb{N}$ tal que $\lambda_{\varepsilon_{n},}>\alpha$ para todo $n \geq n_{1}$. Pelo Teorema do Valor Médio, existe um $c \in\left[\lambda_{\varepsilon_{n}, i}, \lambda_{0 . \imath}\right]$ tal que

$$
e^{-t \lambda_{\varepsilon_{n}, i}}-e^{-t \lambda_{0, i}}=-l e^{-t c}\left(\lambda_{\varepsilon_{n}, i}-\lambda_{0, i}\right) \leq l e^{-t \alpha}\left(\lambda_{0, i}-\lambda_{\varepsilon_{n}, i}\right) \text { para todo } l \in[\beta, \infty) .
$$

A função $h(t):=t e^{-t \alpha}$ possui um ponto de máximo em $t=\alpha^{-1}$. Logo,

$$
\sup _{t \in[\beta, \infty)}\left(e^{-t \lambda_{\varepsilon_{n}, i}}-e^{-t \lambda_{0,2}}\right) \leq(\alpha e)^{-1}\left(\lambda_{0, i}-\lambda_{\varepsilon_{n}, i}\right) \text { para todo } n \geq n_{1},
$$

Como $\lambda_{\varepsilon_{n}, i} \rightarrow \lambda_{0, i}$ quando $n \rightarrow \infty$, obtemos (4.10).

Notemos que $\left|w_{0, i}\right|_{\ell_{n}}^{2}=\lambda_{0, i}+1$ para todo $n \in \mathbb{N}$. Assim, voltando em (4.9) temos que

$$
\sup _{t \in[B, \infty)}\left|P_{n} e^{-A_{\varepsilon_{n}} t} u_{n}-P e^{-A_{0} t} u\right|_{\varepsilon_{n}} \rightarrow 0 \text { quando } n \rightarrow \infty
$$


Analisemos agora a segunda parcela de (4.8). Definimos, para cada $n \in \mathbb{N}, v_{n}:=$ $\left(I-P_{n}\right) e^{-A_{\varepsilon_{n}} t} u_{n}$, onde $t \in[0, \infty)$. Temos que

$$
\begin{aligned}
\left|v_{n}\right|_{\varepsilon_{n}}^{2} & =a_{\varepsilon_{n}}\left(\sum_{i=1}^{\infty}\left\langle v_{n}, w_{\varepsilon_{n}, i}\right\rangle w_{\varepsilon_{n}, i}, \sum_{i=1}^{\infty}\left\langle v_{n}, w_{\bar{\varepsilon}_{n}, i}\right\rangle w_{\varepsilon_{n}, i}\right)+\left|v_{n}\right|_{L^{2}(\Omega)}^{2} \\
& =a_{\varepsilon_{n}}\left(\sum_{i=1}^{\infty}\left\langle v_{n}, w_{\varepsilon_{n}, i}\right\rangle w_{\varepsilon_{n}, i}, \sum_{i=1}^{\infty}\left\langle v_{n}, w_{\bar{\varepsilon}_{n}, i}\right\rangle w_{\varepsilon_{n}, i}\right)+\sum_{i=1}^{\infty}\left|\left\langle v_{n}, w_{\varepsilon_{n}, i}\right\rangle\right|^{2} \\
& =\sum_{i=1}^{\infty} \lambda_{\varepsilon_{n}, i}\left\langle v_{n}, w_{\varepsilon_{n}, i}\right\rangle\left\langle w_{\varepsilon_{n}, i}, \sum_{i=1}^{\infty}\left\langle v_{n}, w_{\varepsilon_{n}, i}\right\rangle w_{\varepsilon_{n}, i}\right\rangle+\sum_{i=1}^{\infty}\left|\left\langle v_{n}, w_{\varepsilon_{n}, i}\right\rangle\right|^{2} \\
& =\sum_{i=1}^{\infty} \lambda_{\varepsilon_{n}, i}\left|\left\langle v_{n}, w_{\varepsilon_{n}, i}\right\rangle\right|^{2}+\sum_{i=1}^{\infty}\left|\left\langle v_{n}, w_{\varepsilon_{n}, i}\right\rangle\right|^{2}=\sum_{i=1}^{\infty}\left(\lambda_{\varepsilon_{n}, i}+1\right)\left|\left\langle v_{n}, w_{\varepsilon_{n}, i}\right\rangle\right|^{2}
\end{aligned}
$$

Para cada $n \in \mathbb{N}$ e cada $i \in \mathbb{N}$, temos que

$$
\begin{aligned}
\left\langle v_{n}, w_{\varepsilon_{n}, i}\right\rangle & =\left\langle e^{-A_{\varepsilon_{n}} t}\left(I-P_{n}\right) u_{n}, w_{\varepsilon_{n}, i}\right\rangle=\left\langle e^{-t_{\varepsilon_{n}} t} \sum_{j=j_{0}}^{\infty}\left\langle u_{n}, w_{\varepsilon_{n}, j}\right\rangle w_{\varepsilon_{n}, j}, w_{\varepsilon_{n}, i}\right\rangle \\
& =\left\langle\sum_{j=j_{0}}^{\infty} e^{-t \lambda_{\varepsilon_{n}, j}}\left\langle u_{n}, w_{\varepsilon_{n}, j}\right\rangle w_{\varepsilon_{n}, j}, w_{\xi_{\xi_{i}, i}}\right\rangle .
\end{aligned}
$$

Portanto,

$$
\begin{aligned}
\left|\left(I-P_{n}\right) e^{-A_{\varepsilon_{n}} t} u_{n}\right|_{\varepsilon_{n}}^{2} & =\sum_{i=j_{0}}^{\infty}\left(\lambda_{\varepsilon_{n}, i}+1\right)\left|\left\langle\sum_{j=j_{0}}^{\infty} e^{-t \lambda_{\varepsilon_{n}, j}}\left\langle u_{n}, w_{\varepsilon_{n}, j}\right\rangle w_{\varepsilon_{n}, j}, w_{\varepsilon_{n}, i}\right\rangle\right|^{2} \\
& =\sum_{j=j_{0}}^{\infty}\left(\lambda_{\varepsilon_{n}, j}+1\right)\left|\left\langle e^{-t \lambda_{\varepsilon_{n}, j}}\left\langle u_{n}, w_{\varepsilon_{n}, j}\right\rangle w_{\varepsilon_{n}, j}, w_{\varepsilon_{n}, j}\right\rangle\right|^{2} \\
& =\sum_{j=j_{0}}^{\infty}\left(\lambda_{\varepsilon_{n}, j}+1\right) e^{-2 t \lambda_{\varepsilon_{n}, j}}\left|\left\langle u_{n}, u_{\varepsilon_{n}, j}\right\rangle\right|^{2} \\
& \leq \sum_{j=j 0}^{\infty} \delta\left|\left\langle u_{n}, w_{\varepsilon_{n}, j}\right\rangle\right|^{2} \leq \delta\left|u_{n}\right|_{L^{2}(\Omega)}^{2} .
\end{aligned}
$$

Como $\left(u_{n}\right)_{n \in \mathbb{N}}$ é uma sequência limitada em $L^{2}(\Omega)$, existe um $C_{1}>0$ tal que

$$
\left|\left(I-P_{n}\right) e^{-A_{\varepsilon_{n}} t} u_{n}\right|_{\varepsilon_{n}}^{2} \leq \delta C_{1} \text { para todo } n \geq n_{1} \text { e todo } t \in[0, \infty) .
$$

Analogamente, ao analisarmos a terceira parcela de (4.8), teremos que existe um $C_{2}>0$ tal que

$$
\left|(I-P) e^{-. \iota_{0} t} u\right|_{\varepsilon_{n}}^{2} \leq \delta C_{2} \text { para todo } t \in[0, \infty)
$$

Como $\delta$ é arbitrário, as desigualdades (4.7), (4.11), (4.12) e (4.13) implicam que

$$
\sup _{t \in[\beta, \infty)}\left|e^{-A_{\varepsilon_{n}} t} u_{n}-e^{-A_{0} t} u\right|_{\tilde{z}_{n}} \rightarrow \infty \text {, quando } n \rightarrow \infty
$$


A demonstração do teorema está concluída.

No resultado de convergência de sernifluxos necessitaremos de algumas estimativas que demonstraremos a seguir.

Lema 4.1.4 Seja $\alpha>0$ e definimos $C(\alpha):=(\alpha / e)^{\alpha}$. Então, para todo $t>0$ e todo $\lambda>0$

$$
\lambda^{\alpha} e^{-\lambda t} \leq C(\alpha) t^{-\alpha}
$$

Demonstração. Dado $\alpha>0$, consideremos a função $h:(0, \infty) \longrightarrow \mathbb{R}$ definida por $h(s):=s^{\alpha} c^{-s}$. Tal função assume valor máximo $\mathrm{cm} s=\alpha$ e, portanto, $s^{\alpha} c^{-s} \leq C(\alpha)$ para todo $s \in(0, \infty)$. Em particular, se $s=\lambda t \operatorname{com} \lambda>0$ e $t>0$, temos que

$$
\lambda^{\prime r} e^{-\lambda l} \leq C(\alpha) t^{-\alpha} .
$$

O lema está demonstrado.

Com ajuda do Lema 4.1.4 demonstraremos o seguinte:

Lema 4.1.5 Para cada $\varepsilon \geq 0$, seja $\left(\lambda_{\varepsilon, j}\right)_{j \in \mathrm{fi}}$ a sequência repetida de autovalores de $A_{\varepsilon}$ e $\left(w_{\varepsilon, j}\right)_{j \in \mathbb{N}}$ um sistema de autovetores $L^{2}(\Omega)$-ortonormal correspondente. Definimos $C_{1}:=$ $(C(1 / 2)+1)^{1 / 2}$ e seja $r>0$. Então valem:

(1) para todo $\varepsilon>0$ e todo $u \in L^{2}(\Omega)$,

$$
\left|e^{-\lambda \mid=r} u\right|_{\leq} \leq\left(C_{1} r^{-1 / 2}+1\right)|u|_{L^{2}(\Omega)}
$$

(2) para todo $u \in I_{i s}^{2}(\Omega)$,

$$
\left|e^{-A_{0} r} u\right|_{\varepsilon} \leq\left(G_{1} r^{-1 / 2}+1\right)|u|_{\delta^{2}(\Omega)}
$$

Demonstração. Sejam $\varepsilon>0$ e $r>0$ e suponhamos que $u \in L^{2}(\Omega)$. Como $\left(w_{\varepsilon, j}\right)_{j \in \mathbb{N}} \dot{c}$ um sistema $L^{\dot{L}}(\Omega)$-ortonormal de autovetores, podemos escrever

$$
u=\sum_{j=1}^{\infty}\left\langle u, w_{\varepsilon_{n}, j}\right\rangle w_{\varepsilon_{n}, \jmath} .
$$

Logo,

$$
\left|e^{-A_{\varepsilon} r} u\right|_{L^{2}(\Omega)}^{2}=\sum_{j=1}^{\infty}\left(e^{-r \lambda_{\varepsilon, j}}\right)^{2}\left|\left\langle u, w_{\varepsilon_{n}, j}\right\rangle\right|^{2}
$$

$$
a_{\varepsilon}\left(e^{-A_{\varepsilon} r} u, e^{-A_{\varepsilon} r} u\right)=\sum_{j=1}^{\approx} \lambda_{\varepsilon, j}\left(e^{-r \lambda_{\varepsilon, j}}\right)^{2} \mid\left\langle u, w_{\varepsilon_{n}, j}\right\rangle^{2} .
$$


Como para cada $j \in \mathbb{N}, \lambda_{\varepsilon, 3} \geq 0$, temos que $e^{-r \lambda_{\varepsilon, j}} \leq 1$ para todo $j \in \mathbb{N}$, e assim

$$
\begin{aligned}
\left|e^{-A_{\varepsilon} r} u\right|_{\varepsilon}^{2} & =\sum_{j=1}^{\infty}\left(\lambda_{\varepsilon, j}+1\right)\left(e^{-r \lambda_{\varepsilon, j}}\right)^{2}\left|\left\langle u, w_{\varepsilon, j}\right\rangle\right|^{2} \\
& \leq \sum_{j=1}^{\infty}\left(\lambda_{\varepsilon, j}^{1 / 2} e^{-r \lambda_{\varepsilon, j}}\right)^{2}\left|\left\langle u, w_{\varepsilon, j}\right\rangle\right|^{2}+\sum_{j=1}^{\infty}\left|\left\langle u, w_{\varepsilon, j}\right\rangle\right|^{2} .
\end{aligned}
$$

Segue do Lema 4.1.4 que $\lambda_{\varepsilon, j}^{1 / 2} e^{-r \lambda_{s, j}} \leq C(1 / 2) r^{-1 / 2}$. Com isso,

$$
\left|e^{-A_{\varepsilon} r} u\right|_{\varepsilon}^{2} \leq \sum_{j=1}^{\infty}\left(C(1 / 2)^{2} r^{-1}\right)\left|\left\langle u, w_{\varepsilon, j}\right\rangle\right|^{2}+\left.\sum_{j=1}^{\infty} \dot{i}\left\langle u, w_{-j, j}\right\rangle\right|^{2}=\left(C(1 / 2)^{2} r^{-1}+1\right)|u|_{L^{2}(\Omega)}^{2} .
$$

Cosequentemente,

$$
\begin{aligned}
\left|e^{-A_{\varepsilon} 7} u\right|_{\varepsilon} & \leq\left(C(1 / 2)^{2} r^{-1}+1\right)^{1 / 2}|u|_{L^{2}(\Omega)}=\left(\left(C(1 / 2)^{2}+1\right) r^{-1}+1\right)^{1 / 2}|u|_{L^{2}(\Omega)} \\
& \leq\left(\left(C(1 / 2)^{2}+1\right)^{1 / 2} r^{-1 / 2}+1\right)|u|_{L^{2}(\Omega)}=\left(C_{1} r^{-1 / 2}+1\right)|u|_{L^{2}(\Omega)}
\end{aligned}
$$

demonstrando (4.15). A demonstraçāo da segunda parte do lema é análoga.

\subsection{Convergência de semifluxos}

Dado $\varepsilon>0$, sejam $\Lambda_{\bar{\varepsilon}}$ o operador setorial gerado pelo par $\left(a_{\varepsilon}, b\right)$ e $A_{0}$ o operador setorial gerado pelo par $\left(a_{0}, b\right)$. Tomando $\alpha=1 / 2$, segue que $X^{\alpha}=H^{1}(\Omega)$ quando $X:=$ $L^{2}(\Omega)$ ॰ $X^{\alpha}=I_{s}^{1}(\Omega)$ quando $X:=L_{s}^{2}(\Omega)$. Scja $U$ um aberto em $H^{1}(\Omega)$ e $\hat{\int}: U \rightarrow L^{2}(\Omega)$ uma aplicação contínua e localmente Lipschitz tal que $\hat{f}\left(U \cap H_{s}^{1}(\Omega)\right) \subset L_{s}^{2}(\Omega)$. Para todo $\varepsilon>0$, a equação de evolução

$$
\dot{u}+A_{\varepsilon} u=\hat{f}(u)
$$

está bem definida. Seja $\pi_{\varepsilon}$ o semifluxo local gerado pelas soluções de (4.17). Também cstá bem definida a equação

$$
\dot{u}+A_{0} u=\hat{f}(u) .
$$

Denotemos por $\pi_{0}$ o semifluxo local gerado pelas soluções de (4.18). Nesta seção analisaremos o que ocorre com a família de semifluxos $\left(\pi_{\varepsilon}\right)_{\varepsilon>0}$ quando $\hat{\varepsilon} \rightarrow 0^{+}$. Iniciamos com o seguinte lema:

Lema 4.2.1 Seja $k$ uma constante positiva e consideremos

$$
\rho(x):=\sum_{n=1}^{\infty} \frac{(k)^{n} x^{n \beta}}{\Gamma(n \beta)}
$$

onde $\beta=1 / 2$ e $\Gamma$ é a função Gama. Então a função $\rho:[0, \infty) \longrightarrow[0, \infty)$ está bem definida e é contínua. 
Demonstração. Fixemos $x_{0} \in[0, \infty)$ e seja $b \in \mathbb{R}$ tal que $x_{0} \in[0, b]$. Com isso,

$$
\frac{(k)^{n} x^{n \beta}}{\Gamma(n \beta)} \leq \frac{\left(k \cdot b^{\beta}\right)^{n}}{\Gamma(n, \beta)} \text { para todo } x \in\lfloor 0, b] .
$$

Afirmamos que a série $\sum_{n=1}^{\infty} \frac{\left(k b^{\beta}\right)^{n}}{\Gamma\left(n^{\beta}\right)}$ é convergente. Supondo que nossa afirmação seja válida, o Teste $M$ de Weicrstrass implica que a série de funções $\sum_{n=1}^{\infty} \frac{(l i)^{n} x^{n \beta}}{\Gamma(n \beta)}$ converge uniformemente em $[0, b]$. Portanto, $\rho$ está bem definida e é contínua neste intervalo e em particular em $x_{0}$. Como $x_{0} \in[0, \infty)$ é qualquer, isso conclui a demonstração do lema. Portanto, basta mostrar que nossa afirmação é verdadeira.

Seja $C>0$ tal que $C^{2}=k^{2} b$. Vamos analisar as séries $\sum_{n=1}^{\infty} a_{n}$ e $\sum_{i=1}^{\infty} \dot{b}_{n}$, onde

$$
a_{n}:=\frac{C^{2 n-1}}{\Gamma\left(\frac{2 n-1}{?}\right)} \text { e } b_{n}:=-\frac{C^{2 n}}{(n-1) !} \text {. }
$$

Se tais séries forem convergentes, por um resultado sobre sequências numéricas (ver, por excmplo, [26]): temos que

$$
\sum_{n=1}^{\infty} a_{n}+\sum_{n=1}^{\infty} b_{n}=\sum_{n=1}^{\infty}\left(a_{n}+b_{n}\right)=a_{1}+b_{1}+a_{2}+b_{2}+\cdots
$$

Além disso, notemos que

$$
\sum_{n=1}^{\infty} \frac{\left(k b^{\beta}\right)^{n}}{\Gamma^{\prime}(\cdots, \beta)}=\sum_{n=1}^{\infty} a_{n}+\sum_{n=1}^{\infty} b_{n}
$$

ou seja, $\sum_{n=1}^{\infty} \frac{\left(k b^{\beta}\right)^{n}}{\Gamma(n \beta)}$ è uma série convergente.

Mostremos que a série $\sum_{n=1}^{\infty} \dot{\theta}_{n}$ converge. Esse fato segue da scguinte igualdade:

$$
\sum_{n=1}^{\infty} b_{n}=\sum_{n=1}^{\infty} \frac{C^{2 n}}{(n-1) !}=C^{2} \sum_{m=0}^{\infty} \frac{C^{2 m}}{m !} .
$$

Já no caso de $\sum_{n=1}^{\infty} a_{n}$, notemos primeiro que para $n>3$,

$$
\Gamma\left(\frac{2 n-1}{2}\right) \geq \Gamma\left(\frac{2 n-2}{2}\right)=\Gamma(n-1) .
$$

Portanto, se $n \geq 3$, temos que

$$
\frac{C^{2 n-1}}{\Gamma\left(\frac{2 n-1}{2}\right)} \leq \frac{C^{2 n-1}}{\Gamma(n-1)}=C \frac{C^{2(n-1)}}{\Gamma(n-1)}=C^{3} \frac{C^{2(n-2)}}{(n-2) !},
$$

e a série $\sum_{m=1}^{\infty} \frac{C^{2 m}}{m !}$ é convergente. Isto conclui a demonstração do lema. 
Estamos em condição de enunciar e demonstrar o principal resultado desse capítulo, a saber, a convergência da família de semifluxos $\left(\pi_{z}\right)_{\varepsilon>0}$ para o semifluxo $\tau_{0}$. Mais precisamente temos:

Teorema 4.2.2 Seja $\left(\varepsilon_{n}\right)_{n \in \mathbb{N}}$ uma sequência de nuimeros positivos tal que $\varepsilon_{n} \rightarrow 0^{+}$quando $n \rightarrow \infty$. Sejam $\left(u_{n}\right)_{n \in \mathbb{N}}$ uma sequência em $H^{1}(\Omega)$ e $u \in H_{s}^{1}(\Omega)$ tais que $\mid u_{n}-u_{j i z i a j j}^{1} \rightarrow 0$ quando $n \rightarrow \infty$. Suponhamos ainda que exista um $b \in(0, \infty)$ tal que $u_{n} \pi_{\varepsilon_{n}} t$ e u $u \pi_{0} t$ estejam definidos para todo $n \in \mathbb{N}$ e todo $t \in[0, b]$. Então, para todo $t \in(0, b]$ e toda sequência $\left(t_{n}\right)_{n \in \mathbb{N}}$ em $(0, b]$ convergindo para $t$, temos que

$$
\left|u_{n} \pi_{\Sigma_{n_{i}}} t_{7 \iota}-u \pi_{0} t\right|_{E_{n}} \rightarrow 0 \text { quando } n \rightarrow \infty
$$

Demonstração. Seja $\left(t_{n}\right)_{n \in \mathbb{N}}$ uma sequência em $(0, b]$ tal que $t_{n} \rightarrow t, t \in(0, b]$. Observemos inicialmente que, para cada $n \in \mathbb{N}$,

$$
\left|u_{n} \pi_{\varepsilon_{1}} t_{n}-u \pi_{0} t\right|_{\varepsilon_{n}} \leq\left|u_{n} \pi_{\varepsilon_{n}} t_{n}-u \pi_{0} t_{n}\right|_{\varepsilon_{n}}+\left|u \pi_{0} t_{n}-u \pi_{0} t\right|_{\varepsilon_{n}}
$$

Como $u \pi_{0} t_{n}, u \pi_{0} t \in H_{s}^{1}(\Omega)$, segue que

$$
\left|u \pi_{0} t_{n}-u \pi_{0} t\right|_{\varepsilon_{n}}=\left|u_{n} \pi_{0} t_{n}-u \pi_{0} t\right|_{H^{1}(\Omega)} \text { para todo } n \in \mathbb{N} \text {. }
$$

Da continuidade do semifluxo $\pi_{0}$ em $I^{1}(\Omega)$ temos que

$$
\left|u \pi_{0} t_{n}-u \pi_{0} t\right|_{H^{1}(\Omega)} \rightarrow 0 \text { quando } n \rightarrow \infty \text {. }
$$

Resta mostrar que $\left|u_{n} \pi_{\varepsilon_{n}} t_{n}-u \pi_{0} t_{n}\right|_{\varepsilon_{n}} \rightarrow 0$ quando $n \rightarrow \infty$. Para cada $t \in[0, b]$, temos pela Fórmula da Variação das Constantes que

$$
\begin{aligned}
u u_{n} \pi_{\varepsilon_{n}} t \cdot u \pi_{0} t & =e^{-A_{\varepsilon_{n}} t} u_{n}-e^{-A_{0} t} u \\
& +\int_{0}^{t} e^{-A_{\varepsilon_{n}}(t-s)} \hat{f}\left(u_{n} \pi_{\varepsilon_{n}} s\right) \mathrm{d} s-\int_{0}^{t} e^{-A_{0}(t-s)} \hat{j}\left(u t \pi_{0} s\right) \mathrm{d} s \\
& =e^{-A_{\varepsilon_{n}} t} u_{n}-e^{-A_{0} t} u \\
& +\int_{0}^{t} e^{-A_{\varepsilon_{n}}(t-s)}\left(\hat{f}\left(u_{n} \pi_{\varepsilon_{n}} s\right) \cdots \hat{f}\left(u \pi_{0} s\right)\right) \mathrm{d} s \\
& +\int_{0}^{i}\left(e^{-A_{\varepsilon_{n}}(t-s)} \hat{f}\left(u \pi_{0} s\right)-e^{-A_{0}(t-s)} \hat{j}\left(u \pi_{0} s\right)\right) \mathrm{d} s .
\end{aligned}
$$

Logo,

$$
\begin{aligned}
\left|u u_{n} \pi_{\varepsilon_{n}} t-u \pi_{0} l\right|_{\varepsilon_{n}} & \leq\left|e^{-A_{\varepsilon_{n}} t} u_{n}-e^{-A_{0} t} u\right|_{\varepsilon_{v_{s}}} \\
& +\int_{0}^{\iota}\left|e^{-A_{\varepsilon_{n}}(t-s)} \hat{f}\left(u \pi_{0} s\right)-e^{-A_{0}(t-s)} \hat{f}\left(u \pi_{0} s\right)\right|_{\varepsilon_{n}} \mathrm{~d} s \\
& +\int_{0}^{t}\left|e^{-A_{\varepsilon_{n}}(t-s)}\left(\hat{f}\left(u_{n} \pi_{\varepsilon_{n}} s\right)-\hat{f}\left(u \pi_{0} s\right)\right)\right|_{\varepsilon_{n}} \mathrm{~d} s .
\end{aligned}
$$


Definimos agora, para cada $n \in \mathbb{N}$, a função $g_{n}:[0, b] \times[0, b] \longrightarrow \mathbb{R}$ como

$$
g_{n}(t, s):= \begin{cases}\left|e^{-A_{\varepsilon_{n}}(t-s)} \hat{f}\left(u \pi_{0} s\right)-e^{-A_{0}(t-s)} \hat{f}\left(u \pi_{0} s\right)\right|_{s_{n}}, & \text { se } 0<s<t, \\ 0, & \text { caso contrário. }\end{cases}
$$

É claro que, para cada $n \in \mathbb{N}, g_{n}$ é contínua cm $O_{1}:=\{(s, t) \in[0, b] \times[0, b] \mid 0<s<t\}$ e em $O_{2}:=\{(s, t) \in[0, b] \times[0, b] \mid 0<t<s\}$. Logo, para cada $n \in \mathbb{N}, g_{n}$ é uma função mensurável em $[0, b] \times[0, b]$. Além disso, $g_{n}$ é integrável em $[0, b] \times[0, b]$ para todo $n \in \mathbb{N}$ Segne do Teorema de Fubini que, para cada $n \in \mathbb{N}$, a função $c_{n}:[0, b] \rightarrow \mathbb{R}$ definida por

$$
c_{n}(t):=\int_{0}^{h} g_{n}(t, s) \mathrm{d} s=\int_{j}^{n} g_{n}(t, s) \mathrm{d} s
$$

está bem definida quase sempre e é mensurável em $[0, b]$.

Para cada $n \in \mathbb{N}$ e todo $t \in[0, b]$, definimos,

$$
a_{n}(t):= \begin{cases}\left|e^{-A_{\varepsilon_{n}} t}-e^{-A_{0} t}\right|_{\hat{\varepsilon}_{n}}+c_{n}(t), & \text { se } t \in(0, b], \\ 0, & \text { quando } t=0 .\end{cases}
$$

Segue que $a_{n}$ é mensurável em $[0, b]$ para todo $n \in \mathbb{N}$. Com isso, (4.21) pode ser reescrita na forma

$$
\left|u_{n} \pi \pi_{\varepsilon_{n}} t-u \pi_{0} t\right|_{\varepsilon_{n}} \leq a_{n}(t)+\int_{0}^{c}\left|e^{-A_{\varepsilon_{n}}(t-s)}\left(\hat{f}\left(u_{n} \pi_{\varepsilon_{n}} s\right)-\hat{f}\left(u \pi_{0} s\right)\right)\right|_{\Sigma_{n}} \mathrm{~d} s .
$$

Segue do Lema 4.1.5 que, se $0<s<t$.

$$
\begin{aligned}
\left|g_{n}(t, s)\right| & \leq\left|e^{-A_{\varepsilon_{n}}(t-s)} \hat{f}\left(u \pi_{0} s\right)\right|_{\varepsilon_{n}}+\left|e^{-A_{0}(t-s)} \hat{f}\left(u \pi_{0} s\right)\right|_{\varepsilon_{n}} \\
& \leq 2 C_{2}\left(C_{1}(t-s)^{-1 / 2}+1\right),
\end{aligned}
$$

onde

$$
C_{2}:=\sup _{s \in[0, b]}\left|\hat{f}\left(u \pi_{0} s\right)\right|_{L^{2}(\Omega)}<\infty .
$$

Recordemos que $\left(t_{n}\right)_{n \in \mathbb{N}}$ é uma sequência em $(0, b]$ convergindo para algum $t \in(0, b]$. Afirmamos que $c_{n}\left(t_{n}\right) \rightarrow 0$ quando $n \rightarrow \infty$. De fato, observemos que, se $0<s<t$, então existe um $n_{0} \in \mathbb{N}$ e um $\beta>0$ tais que $t_{n}-s>\beta$ para todo $n \geq n_{0}$. Assim, pelo Teorema 4.1.3

$$
\begin{aligned}
g_{n}\left(t_{n}, s\right) & =\left|e^{-A_{\varepsilon_{n}}\left(t_{n}-s\right)} \hat{f}\left(u \pi_{0} s\right)-e^{-A_{0}\left(t_{n}-s\right)} \hat{f}\left(u \pi_{0} s\right)\right|_{\varepsilon_{n}} \\
& \leq \sup _{\tau \in[\beta, \infty)}\left|e^{-A_{\varepsilon_{n}} \tau} \hat{f}\left(u \pi_{0} s\right)-e^{-A_{0} \tau} \hat{f}\left(u \pi_{0} s\right)\right|_{\xi_{n}} \rightarrow 0 \text { quando } n \rightarrow \infty .
\end{aligned}
$$

Observemos que neste caso, $0<s<t_{n}$ para todo $n \geq n_{0}$. Logo $\left(t_{n}-s\right)^{-1 / 2} \leq \beta^{-1 / 2}$ para todo $n \geq n_{0}$. Esse fato e a desiguladade (4.23) implicam que

$$
\left|g_{n}\left(t_{n}, s\right)\right| \leq 2 C_{2}\left(C_{1} \beta^{-1 / 2}+1\right)=: C_{3} .
$$


O Teorema da Convergência Dominada de Lebesgue implica que

$$
\lim _{n \rightarrow \infty} \int_{0}^{b} g_{n}\left(t_{n}, s\right) \mathrm{d} s=\int_{0}^{b} \lim _{n \rightarrow \infty} g_{n}\left(t_{n}, s\right) \mathrm{d} s=0 .
$$

Portanto, $c_{n}\left(t_{n}\right) \rightarrow 0$ quando $n \rightarrow \infty$.

Suponhamos agora que $0<t<s$. Então existe um $n_{1} \in \mathbb{N}$ tal que $l_{n}<s$ para todo $n \geq n_{1}$. Portanto $g_{n}\left(t_{n}, s\right)=0$ para todo $n \geq n_{1}$. Novamente

$$
c_{n}\left(t_{n}\right)-\int_{0}^{b} g_{n}\left(t_{n}, s\right) \mathrm{d} s \rightarrow 0 \text { quando } n \rightarrow \infty .
$$

Isso demonstra nossa afirmação

A afirmação demonstrada e o Tcorema 4.1 .3 implicam que

$$
\begin{aligned}
& a_{n}\left(l_{n}\right)=\left|e^{-A_{i, i}^{t} \cdots \underline{l}_{i, i}}-e^{-A_{0} t_{n}} u\right|_{\varepsilon_{n}}+c_{n}\left(t_{n}\right) \\
& \leq \sup _{t \in[\beta, \infty)}\left|e^{-A_{\varepsilon_{n}} t} u_{n}-e^{-A_{0} l} u\right|_{\varepsilon_{n}}+c_{n}\left(l_{n}\right) \rightarrow 0 \text { quando } n \rightarrow \infty .
\end{aligned}
$$

Em particular,

$$
a_{n}(t) \rightarrow 0 \text { para todo } t \in(0, b]
$$

Notemos que a integral imprópria $\int_{j 0}^{t}(t-s)^{-1 / 2} \mathrm{~d} s$ é convergente e que $\int_{0}^{t}(t-s)^{-1 / 2} \mathrm{~d} s=$ $t^{1 / 2}$. Logo,

$$
\begin{aligned}
\int_{0}^{t} g_{n}(l, s) \mathrm{d} s & \leq \int_{J_{0}}^{t} 2 C_{2}\left(C_{1}(t-s)^{-1 / 2}+1\right) \mathrm{d} s \\
& =4 C_{2} C_{1} t^{1 / 2}+2 C_{2} t \leq 4 C_{2} C_{1} b^{1 / 2}+2 C_{2} b=: A_{1}
\end{aligned}
$$

Portanto,

$$
a_{n}(t) \leq\left|e^{-A_{\varepsilon_{n}} t} u_{n}-e^{-A_{0} t} u\right|_{\varepsilon_{n}}+A_{1} \text { para todo } t \in(0, b] .
$$

O Lema 4.1.5 implica que

$$
a_{n}(t) \leq\left(C_{1} t^{-1 / 2}+1\right)\left|u_{n}\right|_{L^{2}(\Omega)}+\left(C_{1} t^{-1 / 2}+1\right)|u|_{L^{2}(\Omega)}+A_{1} .
$$

Como $\left(u_{n}\right)_{n \in \mathbb{N}}$ é uma sequência convergente em $L^{2}(\Omega)$, temos que tal sequência é limitada e, portanto, existe uma constante $A_{2}>0$ tal que

$$
a_{n}(l) \leq\left(C_{1} t^{-1 / 2}+1\right) A_{2}+\left(C_{1} t^{-1 / 2}+1\right) A_{2}+A_{1}
$$

Logo,

$$
a_{n}(t) \leq C_{4}\left(C_{1} t^{-1 / 2}+1\right) \text { para } t \in(0, b],
$$

onde $C_{4}:=\max \left\{C_{1} A_{2}+C_{1} A_{3}, A_{1}+A_{2}+A_{3}\right\}$. 
Novamente, segue do Lema 4.1.5 que, para quaisquer que sejam $t, s \in(0, b] \mathrm{com}$ $t-s>0$

$$
\begin{aligned}
\left|e^{-A_{\varepsilon_{n}}(t-s)}\left(\hat{f}\left(u_{n} \pi_{\varepsilon_{n}} s\right)-\hat{f}\left(u \pi_{0} s\right)\right)\right|_{\varepsilon_{n}} & \leq\left(C_{1}(t-s)^{-1 / 2}+1\right)\left|\hat{f}\left(u_{n} \pi_{\varepsilon_{n}} s\right)-\hat{f}\left(u \pi_{0} s\right)\right|_{L^{2}(\Omega)} \\
& \leq L\left(C_{1}(t-s)^{-1 / 2}+1\right)\left|u_{n} \pi_{\varepsilon_{n}} s-u \pi_{0} s\right|_{H^{1}(\Omega)} \\
& \leq L\left(C_{1}(t-s)^{-1 / 2}+1\right)\left|u_{n} \pi_{\varepsilon_{n}} s-u \pi_{0} s\right|_{\varepsilon_{n}}
\end{aligned}
$$

onde $L>0$ é a constante de Lipschitz de $\hat{f}$ em $U$. Observemos ainda que, neste caso,

$$
\left(C_{1}(t-s)^{-1 / 2}+1\right)=\frac{C+(t-s)^{1 / 2}}{(i-s)^{1 / 2}} \leq \frac{C_{1}+b^{1 / 2}}{(t-s)^{1 / 2}}=c(i-s)^{-1 / 2} .
$$

Logo, para quaisquer que sejam $t, s \in(0 . b] \operatorname{com} t-s>0$,

$$
\left|e^{-A_{\varepsilon_{n}}(t-s)}\left(\hat{f}\left(u_{n} \pi_{\varepsilon_{n}} s\right)-\hat{f}\left(u \pi_{0} s\right)\right)\right|_{\varepsilon_{n}} \leq L C_{5}(t-s)^{-1 / 2}\left|u_{n} \pi_{\varepsilon_{n}} s-u \pi_{0} s\right|_{\varepsilon_{n}} .
$$

Utilizando a desigualdade acima $\mathrm{cm}$ (4.22) obtemos

$$
\left|u_{n} \pi_{\varepsilon_{n}} t-u \pi_{0} t\right|_{\varepsilon_{n}}<a_{n}(t)+\int_{j 0}^{t} L C_{5}(t-s)^{-1 / 2}\left|u_{n} \pi_{\varepsilon_{n}} s-u \pi_{0} s\right|_{\varepsilon_{n}} \mathrm{~d} s .
$$

Notemos que, para todo $n \in \mathbb{N}$, a função $a_{n}$ é não negativa e a desigualdade (4.25) implica que $a_{n}$ é localmente integrável para todo $n \in \mathbb{N}$. Portanto, estamos nas condições do Lema 7.1.1 de [15]. Segue que, para cada $t \in(0, b]$,

$$
\left|u_{n} \pi_{\varepsilon_{n}} t-u \pi_{0} t\right|_{\varepsilon_{n}} \leq a_{n}(t)+\theta \int_{j_{0}}^{i} E_{\beta}^{\prime}(\theta(t-s)) a_{n}(s) \mathrm{d} s,
$$

onde $E_{\beta}(z)=\sum_{n=0}^{\infty} \frac{z^{n \beta}}{\Gamma(n \beta+1)}, \theta=\left(L C_{4} \Gamma(\beta)\right)^{1 / \beta} \operatorname{com} \beta=1 / 2$. Assim, para cada $t \in(0, b]$,

$$
\left|u_{n} \pi_{\varepsilon_{n}} t-u \pi_{0} t\right|_{\varepsilon_{n}} \leq a_{n}(t)+\int_{0}^{t} \tilde{\rho}(t-s) a_{n}(s) \mathrm{d} s
$$

onde

$$
\tilde{\rho}(x)=\sum_{n=1}^{\infty} \frac{\left(L C_{5} \Gamma(\beta)\right)^{n}}{\Gamma(n \beta)} x^{n \beta-1}=\frac{1}{x} \rho(x), \text { para } x \in(0, b],
$$

sendo $\rho$ a função do Lema $4.2 .1 \operatorname{com} k=L_{L} \Gamma(\beta)$. Polo Lcma 4.2 .1 a função $\rho$ está bem definida e é contínua em $(0, b]$. Além disso, para todo $x \in(0, b]$,

$$
\tilde{\rho}(x) \leq L C_{5} x^{-1 / 2}+b^{-1} \sum_{n=2}^{\infty} \frac{\left(L C_{5} \Gamma(\beta) b^{\beta}\right)^{n}}{\Gamma(n \beta)} .
$$

Definindo

$$
C_{6}:=\max \left\{L C_{5}, i^{-i} \sum_{n=2}^{\infty} \frac{\left(L C_{5} \Gamma(\beta) b^{\beta}\right)^{n}}{\Gamma(n \beta)}\right\}
$$


temos que $\tilde{\rho}$ satisfaz a estimativa

$$
\tilde{\rho}(x) \leq C_{6} x^{-1 / 2}+C_{6} \text { para todo } x \in(0, b] .
$$

Afirmamos que

$$
\int_{0}^{t_{n}} \tilde{\rho}\left(t_{n}-s\right) a_{n}(s) \mathrm{d} s \rightarrow 0 \text { quando } n \rightarrow \infty
$$

De fato, fixemos um $\delta_{n}>0$ com $0<\delta_{0}<t$. Dado $\nu>0$, seja $\delta>0$ tal que $0<0<2 \delta<\delta_{0}$ e $\delta^{1 / 2}+\delta<\nu$. Existe um $n_{1} \in \mathbb{N}$ tal que $\left|t_{n}-t\right|<\delta$ para todo $n \geq n_{-}$. Notemos que $l_{n}-s>\partial$ para todo $s \in[0, t-2 \delta]$ e todo $n \geq n_{1}$. Assim, $\tilde{\rho}\left(t_{n}-s\right) \leq C_{6}\left(t_{n}-s\right)^{-1 / 2}+C_{6}<C_{6} \delta^{-1 / 2}+C_{6}$, o que nos fornece a seguinte desigualdade:

$$
\dot{\rho}\left(t_{n}-s\right) a_{n}(s) \leq C_{6}\left(s^{-1 / 2}+1\right) \text { para todo } s \in(0, t-2 \delta] \text { e todo } n \geq n_{1} .
$$

Portanto, se $s \in(0, t-2 \delta], \bar{\rho}\left(t_{n}-s\right) a_{n}(s) \rightarrow 0$ quando $n \rightarrow \infty$ (cf. (4.24)). Estamos então nas condições do 'Teorema da Convergência Dominada de Lebesgue. Logo

$$
\int_{0}^{t-2 \delta} \tilde{\rho}\left(t_{n}-s\right) a_{n}(s) \mathrm{d} s \rightarrow 0 \text { quando } n \rightarrow \infty,
$$

isto é, existe um $n_{0} \in \mathbb{N}$ com $n_{0}>n_{1}$ tal que, para todo $n \geq n_{0}$,

$$
\int_{0}^{t-2 \delta} \tilde{\rho}\left(t_{n}-s\right) a_{n}(s) \mathrm{d} s<\nu
$$

Por outro lado, para todo $s \in\left[t-2 \hat{\delta}, t_{n}\right]$ e todo $n \geq n_{1}$, temos que $s \geq t-2 \delta>t-\delta_{0}$. Segue da desigualdade (4.25) que

$$
a_{n}(s) \leq C_{4}\left(s^{-1 / 2}+1\right) \leq C_{4}\left(\left(t-\delta_{0}\right)^{-1 / 2}+1\right)=: C_{7} .
$$

A desigualdade acima e (4.27) implicam que, para todo $n \geq n_{1}$,

$$
\begin{aligned}
\int_{t-2 \delta}^{t_{n}} \tilde{\rho}\left(t_{n}-s\right) a_{n}(s) \mathrm{d} s & \leq C_{7} \int_{t-2 \delta}^{t_{n}} \tilde{\rho}\left(t_{n}-s\right) \mathrm{d} s \leq C_{7} C_{6} \int_{t-2 \delta}^{t_{n}}\left(\left(t_{n}-s\right)+1\right) \mathrm{d} s \\
& <C_{7} C_{6} \int_{t-2 \delta}^{t_{n}}\left(\delta^{1 / 2}+\delta\right) \mathrm{d} s<C_{8}\left(\delta^{1 / 2}+\delta\right)<C_{8} \nu .
\end{aligned}
$$

Com isso, para todo $n \geq n_{0}$ temos que

$$
\int_{0}^{i n} \tilde{\rho}\left(t_{n}-s\right) a_{n}(s) \mathrm{d} s<\nu+C_{\alpha} \nu .
$$

E nossa afirmação está demonstrada. Isto implica que

$$
\left|u_{n} \pi_{\varepsilon_{n}} t_{n}-u \pi_{0} t_{n}\right|_{\varepsilon_{n}} \rightarrow 0 \text { quando } n \rightarrow \infty \text {. }
$$


As convergências em (4.20) e em (4.28) implicam que

$$
\left|u_{n} \pi_{\bar{\varepsilon}_{n}} t_{n}-u \pi_{0} t\right|_{\varepsilon_{n}} \rightarrow 0 \text { quando } n \rightarrow \infty
$$

A demonstração do teorema está concluída.

Com este resultado, podemos demonstrar um resultado sobre a convergência da família $\left(\sigma_{z}\right)_{\varepsilon>0}$ onde, para cada $\varepsilon>0, \sigma_{\varepsilon}$ é uma solução completa de $\pi_{\varepsilon}$. Demonstramos primeiro o seguinte resultado auxiliar:

Lema 4.2.3 Seja $\left(\varepsilon_{n}\right)_{n \in \mathbb{N}}$ uma sequência arbitrária de números reais tal que $\varepsilon_{n} \rightarrow 0^{+}$ quando $n \rightarrow \infty$. Suponhamos que $\left(u_{n}\right)_{n \in \mathbb{N}}$ seja uma sequência em $H^{1}(\Omega)$ tal que existe uma constante $C>0$ com

$$
\sup _{n \in \mathbb{N}}\left|u_{n}\right|_{\varepsilon_{n}} \leq C
$$

Então existe um $u \in H_{s}^{1}(\Omega)$ tal que $\left|u_{n}-u\right|_{L^{2}(\Omega)} \rightarrow 0$ quando $n \rightarrow \infty$.

Demonstração. Como $\sup _{n \in \mathbb{N}}\left|u_{n}\right|_{\varepsilon_{n}} \leq C$, temos que, para cada $n \in \mathbb{N}$,

$$
\left|u_{n}\right|_{L^{2}(\Omega)} \leq\left|u_{n}\right|_{H^{1}(\Omega)} \leq \sup _{n \in \mathbb{N}}\left|u_{n}\right|_{\varepsilon_{n}} \leq C .
$$

Logo, $\left(u_{n}\right)_{n \in \mathbb{N}}$ é uma sequência limitada $\mathrm{em} H^{1}(\Omega)$. A recíproca do Teorema de EberlianSmulian, (cf. [3], Teorema III.27), implica quc existe uma subsequência de $\left(u_{n}\right)_{n \in \mathbb{N}}$, denotada novamente por $\left(u_{n}\right)_{n \in \mathbb{N}}$, e $u \in H^{1}(\Omega)$ tal que $u_{n} \rightarrow u$. Como $H^{1}(\Omega)$ está contido em $L^{2}(\Omega)$ com inclusão compacta, temos que $\left(u_{n}\right)_{n \in \mathbb{N}}$ converge fortemente em $L^{2}(\Omega)$ para $u \in H^{1}(\Omega)$. Resta mostrar que $u \in I_{s}^{1}(\Omega)$, isto é, que $\partial_{y_{j}} u=0$ para $j=1, \ldots, N$. A desigualdade (4.29) implica que, para cada $n \in \mathbb{N}$,

$$
\int_{\Omega} \nabla_{n} u_{n} \cdot \nabla_{x} u_{n} \mathrm{~d} x \mathrm{~d} y+\frac{1}{\varepsilon_{n}^{2}} \int_{\Omega} \nabla_{y} u_{n} \cdot \nabla_{y} u_{n} \mathrm{~d} x \mathrm{~d} y=a_{\varepsilon_{n}}\left(u_{n} \cdot u_{n}\right) \leq\left|u_{n}\right|_{\varepsilon_{n}}^{2} \leq C^{2} .
$$

Portanto,

$$
\int_{\Omega}^{r} \nabla_{y} u_{n} \cdot \nabla_{y} u_{n} \mathrm{~d} x \mathrm{~d} y \leq \varepsilon_{n}^{2} C^{2} \text { para todo } n \in \mathbb{N}
$$

ou seja, $\left|\partial_{y_{j}} u_{n}\right|_{L^{2}(\Omega)} \rightarrow 0$ quando $n \rightarrow \infty$, para todo $j=1, \ldots, N$. Fixcmos $j=1, \ldots, N$. Para qualquer que seja $\phi \in \mathcal{D}(\Omega)$ temos que

$$
\int_{J_{\Omega}}^{\pi} u \partial_{y_{j}} \phi \mathrm{d} x \mathrm{~d} y=\int_{\Omega}\left(u-u_{n}+u_{n}\right) \partial_{y_{j}} \phi \mathrm{d} x \mathrm{~d} y=\int_{\Omega}\left(u-u_{n}\right) \partial_{y_{j}} \phi \mathrm{d} x \mathrm{~d} y+\int_{\Omega} u_{n} \partial_{u_{j}} \phi \mathrm{d} x \mathrm{~d} y
$$

A Desigualdade de Hölder implica que

$$
\int_{\Omega} u \partial_{y}, \phi \mathrm{d} x \mathrm{~d} y \leq\left|u_{n}-u\right|_{L^{2}(\Omega)}\left|\partial_{y_{j}} \phi\right|_{L^{2}(\Omega)}+\left|\partial_{y_{j}} u_{n}\right|_{L^{2}(\Omega)}|\phi|_{L^{2}(\Omega)}
$$

Fazendo $n \rightarrow \infty$, obtemos que $\partial_{y_{j}} u=0$ para $j=1, \ldots, N$, e, portanto, $u \in H_{s}^{1}(\Omega)$.

Uma consequência do teorema que acabamos de demonstrar é o seguinte resultado de convergência das soluções: 
Corolário 4.2.4 Seja $\left(\varepsilon_{n}\right)_{n \in \mathbb{N}}$ uma sequência arbitrária de números tal que $\varepsilon_{n} \rightarrow 0^{+}$ quando $n \rightarrow \infty$. Para cada $n \in \mathbb{N}$, seja $\sigma_{n}: \mathbb{R} \rightarrow H^{1}(\Omega)$ uma solução completa de $\pi_{\varepsilon_{n}}$. Assumimos que existe uma constante $C \in[0, \infty)$ tal que

$$
\sup _{t \in \mathbb{R}}\left|\sigma_{n}(t)\right|_{\varepsilon_{n}} \leq C \text {. }
$$

Assumimos também que $\hat{f}$ seja uma função Lipschitz em $B_{C}:=\left\{\left.u \in H^{1}(\Omega)|| u\right|_{H^{1}(\Omega)} \leq\right.$ $C\}$ e que, se $u \in B_{C}$, então $u \pi_{0} t$ está definido para todo $t \in[0, \infty)$.

Então existe uma subsequência de $\left(\sigma_{n}\right)_{n \in \mathbb{N}}$, denotada novamente por $\left(\sigma_{n}\right)_{n \in \mathbb{N}}$, e uma solução competa $\sigma: \mathbb{R} \rightarrow H_{s}^{1}(\Omega)$ do semifluxo $\pi_{0}$ tal que

$$
\left|\sigma_{n}(t)-\sigma(i)\right|_{c_{n}} \rightarrow 0 \text { quando } n \rightarrow \infty \text { para todo } t \in \mathbb{R}
$$

Demonstração. Para cada $k \in \mathbb{N}$, a sequência $\left(\sigma_{n}(-k)\right)_{n \in \mathbb{N}}$ está nas condições do Lema 4.2.3. Portanto, para cada $k \in \mathbb{N}$, existe um $u_{-k} \in H_{s}^{1}(\Omega)$ e uma subsequência de $\left(\sigma_{n}(-k)\right)_{n \in \mathbb{N}}$, denotada novamente por $\left(\sigma_{n}(-k)\right)_{n \in \mathbb{N}}$, tal que $\left|\sigma_{n}(-k)-u_{-k}\right|_{L^{2}(\Omega)} \rightarrow 0 \mathrm{e}$ $\sigma_{n}(-k) \rightarrow u_{-k}$ ein $H^{1}(\Omega)$ quando $n \rightarrow \infty$.

Utilizando o Procedimento da Diagonal de Cantor, existe uma subsequência de $\left(\sigma_{n}\right)_{n \in \mathbb{N}}$ denotada novamente por $\left(\sigma_{n}\right)_{n \in \mathbb{N}}$, tal que, para cada $k \in \mathbb{N}$,

$$
\begin{gathered}
\sigma_{n}(-k) \rightarrow u_{-k} \text { em } H^{1}(\Omega) \text { quando } n \rightarrow \infty, \\
\left|\sigma_{n}(-k)-u_{-k}\right|_{L^{2}(\Omega)} \rightarrow 0 \text { quando } n \rightarrow \infty .
\end{gathered}
$$

Além disso, $\left|u_{-k}\right|_{H^{1}(\Omega)} \leq C$ para todo $k \in \mathbb{N}$.

Sejam $k \in \mathbb{N}$ e $t \in[-k, \infty)$. Como $u_{-k} \pi_{0}(t+k)$ está definido, podemos definir

$$
\tau_{k}(t):=u_{-k} \pi_{0}(t+k) \text { para todo } t \in[-k, \infty) \text {. }
$$

Como

$$
\sigma_{n}(t)=\sigma_{n}(-k) \pi_{\varepsilon_{n}}(t+k) \text { para todo } k \in \mathbb{N} \text { e todo } l \in[-k ; \infty),
$$

o Teorema 4.2.2 implica que

$$
\left|\sigma_{n}(t)-\tau_{k}(t)\right|_{\varepsilon_{n}}=\left|v(-k) \pi_{0}(t+k)-\sigma_{n}(-k) \pi_{\tilde{\varepsilon}_{n}}(t+k)\right|_{\varepsilon_{n}} \rightarrow 0 \text { quando } n \rightarrow \infty .
$$

Em particular, se $l, k \in \mathbb{N} \operatorname{com} l>k$, temos que, para $t \in[-k, \infty)$,

$$
\begin{aligned}
\left|\tau_{l}(t)-\tau_{k}(t)\right|_{H^{1}(\Omega)} & =\left|\tau_{l}(t)-\tau_{k}(t)\right|_{\varepsilon_{n}} \\
& \leq\left|\tau_{l}(t)-\sigma_{n}(t)\right|_{\varepsilon_{n}}+\left|\tau_{k}(t)-\sigma_{n}(t)\right|_{\varepsilon_{i k}} \rightarrow 0 \text { quando } n \rightarrow \infty .
\end{aligned}
$$

Ou seja, $\tau_{l}(t)=\tau_{k}(t)$ para todo $t \in[-k, \infty)$. Segue que existe uma única função $\sigma: \mathbb{R} \rightarrow$ $H_{s}^{1}(\Omega)$ tal que $\sigma(t)=\tau_{k}(t), t \in[-k, \infty)$. Consequentemente, $\sigma$ é uma solução de $\pi_{0}$ tal que, para todo $t \in \mathbb{R}$

$$
\left|\sigma_{n}(t)-\sigma(t)\right|_{\varepsilon_{n}} \rightarrow 0 \text { quando } n \rightarrow \infty
$$

A demonstração está completa

No próximo capítulo o Corolário acima sorá utilizado na domonstragăa da somicontinuidade superior da família de atratores globais. 


\section{Capítulo 5}

\section{Semicontinuidade Superior dos Atratores}

Na primeira seção deste capítulo mostraremos a existência de atratores globais para os semifluxos $\pi_{\varepsilon}, \varsigma \geq 0$, gerados pelas soluções da equação de evolução

$$
\dot{u}+A_{\varepsilon} u=\hat{f}(u),
$$

impondo condições adicionais em $f$, além da hipótese (HC1). Para cada $\varepsilon \geq 0$, obteremos um atrator global $\mathcal{A}_{\pi_{\tilde{r}}}$. Estamos interessados no comportamento da família $\left(\mathcal{A}_{\pi_{\varepsilon}}\right)_{\varepsilon>0}$ quando $\varepsilon \rightarrow 0^{+}$. Na Seção 5.2 analisaremos este comportamento. Mais precisamente, apresentaremos um resultado de semicontinuidade superior da família $\left(\mathcal{A}_{\pi_{\epsilon}}\right)_{\varepsilon>0}$ quando $\varepsilon \rightarrow 0^{+}$. Utilizaremos a notação apresentada no Capítulo 2, em particular, das Seções 2.1 e 2.3

\subsection{Existência de atratores}

Scja $f: \mathbb{R} \longrightarrow \mathbb{R}$ uma função satisfazendo a hipótesc (HC1), isto ć, $f \in C^{1}(\mathbb{R})$ e existem constantes $C, \beta \in[0, \infty)$ tais que $\left|f^{\prime}(s)\right| \leq C\left(|s|^{\hat{\beta}}+1\right)$ para todo $s \in \mathbb{R}$. Além disso, se $M+N=: n>2$, suponhamos também que $\beta \leq 2^{*} / 2-1$, onde $2^{*}=2 n /(n-2)>$ 2

Definimos $F: \mathbb{R} \longrightarrow \mathbb{R}$ como

$$
F(y):=\int_{0}^{y} f(s) \mathrm{d} s \text { para todo } y \in \mathbb{R} .
$$

Teorema 5.1.1 Seja $\hat{F}$ o operador de Nemitskii associado a $F$. Então $\hat{F}\left(I^{1}(\Omega)\right) \subset$ $L^{1}(\Omega), \hat{F}$ aplica subconjuntos limitados de $H^{1}(\Omega)$ em subconjuntos limitados de $L^{1}(\Omega)$ e é Fréchet-diferenciável em $H^{1}(\Omega)$ com $D \hat{F}(u)(v)=\hat{\jmath}(u)$.v para quaisquer que sejam u e $v$ em $H^{1}(\Omega)$.

Demonstração. Pela definição de $F$ e pcla Desiguladade de Young, existem constantes $K_{1}, K_{2} \in(0, \infty)$ tais que

$$
|F(y)| \leq K_{1}|y|^{\beta+2}+K_{2} \text { para todo } y \in \mathbb{R} .
$$


Logo, $\dot{F}(u) \in L^{1}(\Omega)$ para todo $u \in L^{\beta+2}(\Omega)$. Como $\beta \leq 2^{*} / 2-1$, segue que $\beta+2 \leq$ $2^{*}$ e assim $H^{1}(\Omega)$ está continuamente imerso em $L^{\beta+2}(\Omega)$ e $\hat{F}(u) \in L^{1}(\Omega)$ para todo $u \in H^{1}(\Omega)$, ou seja, o operador de Nemitskiĭ $\hat{F}: H^{1}(\Omega) \longrightarrow L^{1}(\Omega)$ está bem definido.

Nostremos que a imagem de subconjuntos limitados de $H^{1}(\Omega)$ pelo operador $\hat{F}$ é também um subconjunto limitado $L^{\mathrm{I}}(\Omega)$. Integrando em $\Omega$ ambos os lados de (5.2) obtemos:

$$
|\hat{F}(u)|_{L^{1}(\Omega)} \leq K_{3}\left(|u|_{L^{\beta+2(\Omega)}}^{\beta+2}+1\right) \text { para todo } u \in H^{1}(\Omega),
$$

onde $K_{3}=\max \left\{K_{1}, K_{2} \mu(\Omega)\right\}>0$. Usando que $H^{1}(\Omega)$ está continuamente imerso em $L_{L}^{\beta+2}(\Omega)$ temos

$$
|\hat{F}(u)|_{L^{1}(\Omega)} \leq K_{3}\left(\left.||_{u}\right|_{H^{\prime}} ^{2} i_{(\Omega)}^{2}+1\right) \text { para todo } u \in H^{1}(\Omega) .
$$

Obscrvemos que $F^{\prime \prime}=f$ e como $\mu(\Omega)<\infty$, temos que as aplicaçõcs

$$
\hat{F}: L^{2(3+1)}(\Omega) \longrightarrow L^{1}(\Omega) \text { e } \hat{f}: L^{2(\beta+1)}(\Omega) \longrightarrow L^{2}(\Omega)
$$

estão bem definidas. O Teorema 2.6 de [10] implica que $\hat{F}$ é Fréchet-diferenciável em $L^{2(\beta+1)}(\Omega) \operatorname{com} D \hat{F}(u) \cdot v=\hat{f}(u) . v$, quaisquer que sejam $u, v \in L^{2(\beta+1)}(\Omega)$. Como $H^{1}(\Omega)$ está imerso em $L^{2(\beta+1)}(\Omega)$, o resultado segue.

Seja $F$ definida por (5.1). Para cada $\varepsilon>0$, definimos $\mathcal{L}_{\varepsilon}: H^{1}(\Omega) \longrightarrow \mathbb{R}$ por

$$
\mathcal{L}_{\dot{s}}(u):=\frac{1}{\tau} a_{\varepsilon}(u, u)-\int_{\Omega} \hat{F}(u) \mathrm{d} x \mathrm{~d} y,
$$

e $\mathcal{L}_{0}: H_{s}^{1}(\Omega) \longrightarrow \mathbb{R}$ por

$$
\mathcal{L}_{0}(u):=\frac{1}{2} a_{0}(u, u)-\int_{\Omega} \hat{F}(u) \mathrm{d} x \mathrm{~d} y
$$

Lema 5.1.2 Para cada $\varepsilon \geq 0$, o semifluxo global $\pi_{\varepsilon}$ é do tipo gradiente com relação à funç̧ão $\mathcal{L}_{\varepsilon}$.

Demonstração. O Teorema 5.1.1 implica que $\mathcal{L}_{\varepsilon}$ é Fréchet-diferenciável para cada $\varepsilon \geq 0$ e, em particular, contínua. Fixemos $\varepsilon>0$. Pelo Exemplo 1.2.2 temos que, se $\sigma: J \longrightarrow$ $H^{1}(\Omega)$ é uma solução não-constante de $\pi_{\varepsilon}$, então $\sigma$ é diferenciável em $J$. Mostremos que $\mathcal{L}_{\varepsilon} \circ \sigma$ é estritamente decrescente em $J$. Para isto, definimos

$$
G(v):=\int_{\Omega} v \mathrm{~d} x \mathrm{~d} y \text { qualquer que seja } v \in L^{1}(\Omega)
$$

Assim

$$
\mathcal{L}_{\varepsilon}(u)=\frac{1}{2} a_{\varepsilon}(u, u)-G \circ \hat{F}(u) .
$$


Sabemos pela Regra da Cadeia que

$$
\left(\mathcal{L}_{\varepsilon} \circ \sigma\right)^{\prime}(t)=\mathcal{L}_{\varepsilon}^{\prime}(\sigma(t)) \cdot \sigma^{\prime}(t) \text { para todo } t \in J
$$

Por outro lado, se $h \in H^{1}(\Omega)$; temos pela Regra da Cadeia que, para todo $u \in H^{1}(\Omega)$,

$$
\mathcal{L}_{\varepsilon}^{\prime}(u) \cdot h=a_{\varepsilon}(u, h)-G^{\prime}(\hat{F}(u)) \circ \hat{F}^{\prime}(u) \cdot h=a_{\varepsilon}(u, h)-G(\hat{f}(u) \cdot h) .
$$

Logo,

$$
\left(\mathcal{L}_{\varepsilon} \circ \sigma\right)^{\prime}(t)=a_{\varepsilon}\left(\sigma(t), \sigma^{\prime}(t)\right)-\int_{\Omega} \hat{f}(\sigma(t)) \sigma^{\prime}(t) \mathrm{d} x \mathrm{~d} y \text { para todo } t \in J .
$$

Como $\sigma$ é solução de $\pi_{\varepsilon}$, temos que $\hat{f}(\sigma(t))=\sigma^{\prime}(t)+A_{\varepsilon} \sigma(t)$ para todo $t \in J$, e assim,

$$
\begin{aligned}
\int_{\Omega} \hat{f}(\sigma(t)) \sigma^{\prime}(t) \mathrm{d} x \mathrm{~d} y & =\int_{\Omega}|\sigma(t)|^{2} \mathrm{~d} x \mathrm{~d} y+\int_{\Omega}\left(A_{\varepsilon} \sigma(t)\right) \sigma^{\prime}(t) \mathrm{d} x \mathrm{~d} y \\
& =\left|\sigma^{\prime}(t)\right|_{L^{2}(\Omega)}^{2}+\left\langle A_{\varepsilon} \sigma(t), \sigma^{\prime}(t)\right\rangle \\
& =\left|\sigma^{\prime}(t)\right|_{L^{2}(\Omega)}^{2}+a_{\varepsilon}\left(\sigma(t), \sigma^{\prime}(t)\right) .
\end{aligned}
$$

Assim,

$$
\left(\mathcal{L}_{\varepsilon} \circ \sigma\right)^{\prime}(t)=-\left|\sigma^{\prime}(t)\right|_{L^{2}(\Omega)}^{2} \leq 0 \text { para todo } t \in J .
$$

Em particular, se $u \in H^{1}(\Omega) \backslash E$, onde $E$ é o conjunto dos pontos de equilíbrio de $\pi_{\varepsilon}$, Segue que a função $t \mapsto \mathcal{L}_{\varepsilon}(\sigma(t)), t \in J$ é estritamente decrescente. O caso $\varepsilon=0$ possui demonstração análoga.

Lema 5.1.3 Dado $C \in[0, \infty)$, definimos

$$
\theta=\theta(C):=\sup _{|u|_{H^{1}(\Omega)} \leq C}|\hat{F}(u)|_{L^{1}(\Omega)}<\infty .
$$

(1) Para todo $\varepsilon \in(0,1]$ e $u \in I^{1}(\Omega)$ tal que $|u|_{\varepsilon} \leq C$, temos que $\mathcal{L}_{\varepsilon}(u) \leq \frac{1}{\overline{2}}|u|_{\varepsilon}^{2}+\theta$;

(2) para todo $u \in H_{s}^{1}(\Omega)$ tal que $|u|_{H^{1}(\Omega)} \leq C$, segue que $\mathcal{L}_{0}(u) \leq \frac{1}{2}|u|_{H^{1}(\Omega)}^{2}+\theta$.

Demonstração. Mostremos (1). Suponhamos que $u \in H^{1}(\Omega)$ scja tal que $|u|_{H^{1}(\Omega)} \leq C$. Então

$$
\left|\int_{\Omega} \hat{F}(u) \mathrm{d} x \mathrm{~d} y\right| \leq \int_{\Omega}|\hat{F}(u)| \mathrm{d} x \mathrm{~d} y \leq \theta
$$

Como $\varepsilon \in(0,1]$, a Proposição 2.3 .4 implica que $|u|_{H^{1}{ }^{1}(\Omega)} \leq|u|_{\xi}$. Segue que $|u|_{\mu 1(\Omega)} \leq C$. Utilizando (5.3) obtemos

$$
\begin{aligned}
\mathcal{L}_{\varepsilon}(u) & =\frac{1}{2} a_{\varepsilon}(u, u)-\int_{\Omega} \hat{F}(u) \mathrm{d} x \mathrm{~d} y<\frac{1}{2} a_{\varepsilon}(u, u)+\theta \\
& \leq \frac{1}{2} a_{\varepsilon}(u, u)+\frac{1}{2}|u|_{L^{2}(\Omega)}^{2}+\theta=\frac{1}{2}|u|_{\varepsilon}^{2}+\theta .
\end{aligned}
$$


Seja agora $u \in H_{s}^{1}(\Omega)$ tal que $|u|_{H^{1}(\Omega)} \leq C$. Utilizando (5.3) temos que

$$
\begin{aligned}
\mathcal{L}_{0}(u) & =\frac{1}{2} a_{0}(u, u)-\int_{\Omega} \hat{F}(u) \mathrm{d} x \mathrm{~d} y \\
& \leq \frac{1}{2} \int_{\Omega} \nabla_{x} u \cdot \nabla_{x} u \mathrm{~d} x \mathrm{~d} y+\frac{1}{2}|u|_{L^{2}(\Omega)}^{2}+\theta=\frac{1}{\overline{2}}|u|_{L I !(\Omega)}^{2}+\theta .
\end{aligned}
$$

A demonstração do lema está concluída.

Para continuarmos, vamos assumir que a não-linearidade $f$ satisfaça, além de (HC1), a seguinte condição de dissipatividade:

(HC2) existe um $\xi>0$ tal que $\varlimsup_{|s| \rightarrow \infty} \frac{f(s)}{s}<-\xi$.

Portanto, dado $\eta>0$, existe um $M=M(\eta)>0$ tal que

$$
\frac{f(s)}{s} \leq-\xi+\eta \text { para todo } s \in \mathbb{R} \text { com }|s|>M
$$

Logo,

$$
f(s) s \leq(-\xi+\eta) s^{2} \text { para todo } s \in \mathbb{R} \operatorname{com}|s|>M
$$

Como

segue que

$$
F(s)=\int_{0}^{s} f(t) \mathrm{d} t \text { para todo } s \in \mathbb{R}
$$

$$
F(s)=\int_{0}^{\ddot{\text { ii }}} f(t) \mathrm{d} t+\int_{M}^{s} \frac{1}{t} f(t) t \mathrm{~d} t \text { para todo } s \in \mathbb{R}
$$

A função $f$ é contínua em $[-M, M]$. Logo, existe um $c_{1}=c_{1}(M)>0$ tal que $f(t) \leq c_{1}$, para todo $s \in[-M, M]$. Portanto,

$$
\begin{aligned}
F(s) & \leq c_{1} M+\int_{J_{M}}^{s} \frac{1}{i}(-\xi+\eta) t^{2} \mathrm{~d} t=c_{1} M+\frac{1}{i}(-\xi+\eta) s^{2}-\frac{1}{\dot{2}}(-\xi+\eta) M^{2} \\
& =\frac{1}{2}(-\xi+\eta) s^{2}+c_{2} \text { para todo } s \in \mathbb{R} \text { com }|s|>M
\end{aligned}
$$

onde $c_{2}=c_{2}(\eta):=c_{1} M-\frac{1}{2}(-\xi+\eta) M^{2}$.

Por outro lado, para $s \in \mathbb{R}$ com $|s| \leq M$, existem constantes $c_{3}=c_{3}(\eta)$ e $c_{4}=c_{4}(\eta)$ tais que

$$
\begin{aligned}
& f(s) s-(-\xi+\eta) s^{2} \leq c_{3}, \\
& F(s)-\frac{1}{2}(-\xi+\eta) s^{2} \leq c_{4},
\end{aligned}
$$

já que ambas as funções do lado dircito das desigualdades acima são contínuas em $[-M, M]$. 
Assim, tomando $c^{\prime}=c^{\prime}(\eta):=\max \left\{c_{2}, c_{3}, c_{4}\right\}$, temos que

$$
\begin{aligned}
& f(s) s \leq(-\xi+\eta) s^{2}+c^{\prime} \text { para todo } s \in \mathbb{R}, \\
& F(s) \leq(-\xi+\eta) s^{2}+c^{\prime} \text { para todo } s \in \mathbb{R} .
\end{aligned}
$$

Utilizaremos as desigualdades (5.4) e (5.5) no que segue.

Lema 5.1.4 Existe uma constante $K>0$ tal que

$$
\begin{gathered}
|u|_{\varepsilon} \leq K\left(\mathcal{L}_{\varepsilon}(u)+1\right) \text { para todo } u \in H^{1}(\Omega) \text { e todo } \varepsilon \in(0,1], \\
|u|_{I^{1}(\Omega)} \leq K\left(\mathcal{L}_{0}(u)+1\right) \text { para todo } u \in H_{s}^{1}(\Omega) .
\end{gathered}
$$

Demonstração. Fixemos $\eta \in \mathbb{R}$ tal que $0<\eta<\xi$. Utilizando (5.5) temos que, para todo $\varepsilon \in(0,1]$ e todo $u \in H^{1}(\Omega)$ :

$$
\begin{aligned}
\mathcal{L}_{\varepsilon}(u) & =\frac{1}{2} a_{\varepsilon}(u, u)-\int_{\Omega} \hat{F}(u) \mathrm{d} x \mathrm{~d} y \\
& \geq \frac{1}{2} a_{\varepsilon}(u, u)-\frac{1}{2}(-\xi+\eta) \int_{\Omega}|u|^{2} \mathrm{~d} x \mathrm{~d} y-c^{\prime}(\eta) \int_{\Omega} \mathrm{d} x \mathrm{~d} y \\
& =\frac{1}{2} a_{\varepsilon}(u, u)+\frac{1}{2}(\xi-\eta)|u|_{L^{2}(\Omega)}^{2}-c^{\prime}(\eta) \mu(\Omega) .
\end{aligned}
$$

Para todo $u \in H_{s}^{1}(\Omega)$,

$$
\mathcal{L}_{0}(u)=\frac{1}{2} a_{0}(u, u)-\int_{\Omega} \hat{F}(u) \mathrm{d} x \mathrm{~d} y \geq \frac{1}{2} a_{0}(u, u)+\frac{1}{2}(\xi-\eta)|u|_{L^{2}(\Omega)}^{2}-c^{\prime}(\eta) \mu(\Omega) .
$$

Definimos $c:=c^{\prime}(\eta) \mu(\Omega)$ e $k:=\frac{1}{2} \min \{1, \xi-\eta\}$. Para todo $\varepsilon \in(0,1]$, obtcmos que

$$
\mathcal{L}_{\varepsilon}(u) \geq k a_{\varepsilon}(u, u)+k|u|_{L^{2}(\Omega)}^{2}-c^{\prime}(\eta) \mu(\Omega)=k|u|_{\varepsilon}^{2}-c .
$$

Ou seja,

$$
|u|_{\Sigma}^{2} \leq \frac{1}{k}\left(\mathcal{L}_{f}(u)+c\right) \text { para todo } u \in H^{1}(\Omega) \text { e todo } \varepsilon \in(0,1] .
$$

Analogamente mostramos que, para todo $u \in H_{s}^{1}(\Omega)$.

$$
|u|_{H^{1}(\Omega)}^{2} \leq \frac{1}{k}\left(\mathcal{L}_{0}(u)+c\right)
$$

Assim,

$$
\begin{aligned}
|u|_{\Sigma} \leq & \left(\frac{1}{k}\right)^{1 / 2}\left(\mathcal{L}_{\varepsilon}(u)+c\right)^{1 / 2} \text { para todo } u \in H^{1}(\Omega) \text { e todo } \varepsilon \in(0,1], \\
& |u|_{H^{1}(\Omega)} \leq\left(\frac{1}{k}\right)^{1 / 2}\left(\mathcal{L}_{0}(u)+c\right)^{1 / 2} \text { para todo } u \in H_{s}^{1}(\Omega) .
\end{aligned}
$$


Pela Desigualdade de Young segue que

$$
\begin{aligned}
&|u|_{\varepsilon} \leq \frac{1}{2 k}+\frac{1}{2}\left(\mathcal{L}_{\varepsilon}(u)+c\right) \text { para todo } u \in I^{1}(\Omega) \text { c todo } \varepsilon \in(0,1], \\
&|u|_{H^{1}(\Omega)} \leq \frac{1}{2 k}+\frac{1}{2}\left(\mathcal{L}_{n}(u)+c\right) \text { para todo } u \in H_{s}^{1}(\Omega) .
\end{aligned}
$$

Definindo $K:=\max \{1 / 2 k+c / 2,1 / 2\}$, temos que

$$
\begin{aligned}
|u|_{\varepsilon} \leq & K\left(\mathcal{L}_{\varepsilon}(u)+1\right), \text { para todo } u \in H^{1}(\Omega) \text { e todo } \varepsilon \in(0,1], \\
& |u|_{H^{1}(\Omega)} \leq K\left(\mathcal{L}_{0}(u)+1\right), \text { para todo } u \in H_{s}^{1}(\Omega) .
\end{aligned}
$$

O lema cstá demonstrado.

Lema 5.1.5 Seja $\varepsilon \in[0,1]$ e u um ponto de equlíbrio de $\pi_{\varepsilon}$. Então, se $\varepsilon \in(0,1]$.

$$
|u|_{\varepsilon}^{2} \leq \frac{\kappa}{h}
$$

Se $\varepsilon=0$,

$$
|u|_{H^{1}(\Omega)}^{2} \leq \frac{c}{k}
$$

Demonstração. Seja $u$ um ponto de equilíbrio de $\pi_{\varepsilon}, \varepsilon \in[0,1]$. Então $u \in D\left(A_{\varepsilon}\right)$ e $A_{\varepsilon} u=\hat{f}(u)$. Usando (5.4) obtemos

$$
\begin{aligned}
a_{\varepsilon}(u, u) & =\left\langle A_{\varepsilon} u, u\right\rangle=\langle\hat{f}(u), u\rangle=\int_{\Omega} f(u) u \mathrm{~d} x \mathrm{~d} y \\
& \leq \int_{\Omega}\left((-\xi+\eta)|u|^{2}+c^{\prime}\right) \mathrm{d} x \mathrm{~d} y=(-\xi+\eta)|u|_{L^{2}(\Omega)}^{2}+c
\end{aligned}
$$

isto é,

$$
a_{\varepsilon}(u, u)+(\xi-\eta)|u|_{L^{2}(\Omega)}^{2} \leq c .
$$

Lembremos que $k \leq \xi-\eta$ e $k \leq 1$. Portanto,

$$
k|u|_{\varepsilon}^{2} \leq k a_{\varepsilon}(u, u)+k|u|_{L^{2}(\Omega)}^{2} \leq a_{\bar{\varepsilon}}(u, u)+(\xi-\eta)|u|_{L^{2}(\Omega)}^{2} \leq c,
$$

e segue (5.10). Analogamente demonstramos a desigualdade (5.11).

Estes resultados demonstrados nos possibilita apresentar o seguinte resultado sobre a existência de atratores globais:

Teorema 5.1.6 Suponhamos que a função f satisfaça (HC1) e (HC2) e seja $\varepsilon \in[0,1]$. Então o semifluxo local $\pi_{\varepsilon}$ em $H^{1}(\Omega)$ é um semifluxo global em $H^{1}(\Omega)$ para cada $\varepsilon>0 e$ o semifluxo local $\pi_{0}$ em $H_{i}^{1}(\Omega)$ é um semifluxo global em $H_{s}^{1}(\Omega)$. Além disso, o conjunto $\mathcal{A}_{\pi_{\varepsilon}}$ de todas as órbitas limitadas de $\pi_{\varepsilon}$ é um atrator global em $H^{1}(\Omega)$ para $\varepsilon>0$ e em $H_{s}^{1}(\Omega)$ se $\varepsilon=0$.

Demonstração. O Teorema 2.1.8, os Lemas 5.1.2, 5.1.3 e 5.1.5 juntamente com as desigualdades (5.6) e (5.7) implicam que $\hat{f}$ e $\pi_{\varepsilon}, \varepsilon \in[0,1]$, satisfazem todas as hipóteses do Teorema 1.3.2. 


\subsection{Semicontinuidade superior dos atratores}

Nessa seção vamos assumir que as hipóteses do Teorema 5.1.6 estejam satisfeitas. Nossos esforços agora serāo para mostrar um resultado de semicontinuidade superior em $\varepsilon=0$ da fanuília de atratores $\left(\mathcal{A}_{\pi_{\varepsilon}}\right)_{\varepsilon \in[0,1]}$ com relaşão à família de normas $|\cdot|_{\varepsilon}$. Começamos com um lema auxiliar.

Lema 5.2.1 Seja $\theta=\theta(c / k)$ como no Lema 5.1.3. Definimos

$$
K^{\prime \prime}:=(c / 2)(1-(\xi-\eta) / k)+\theta \in(0, \infty) .
$$

Então

$$
\begin{aligned}
& \sup _{\varepsilon \in((1), B]} \sup _{u \in \mathcal{A}_{\pi_{0}}}|u|_{\Xi}^{2} \leq\left(\frac{1}{k}\right)\left(K^{\prime}+c\right) \\
& \sup _{u \in \mathcal{A}_{\pi_{0}}}|u|_{H^{1}(\Omega)}^{2} \leq\left(\frac{1}{k}\right)\left(K^{\prime}+c\right) .
\end{aligned}
$$

Demonstração. Seja $\varepsilon \in(0,1]$ e $u \in \mathcal{A}_{\pi_{\varepsilon}}$ arbitrário. Como $\mathcal{A}_{\pi_{\varepsilon}}$ é invariante, existe uma soluçãa completa $\sigma_{\bar{\varepsilon}}$ de $\pi_{\bar{\varepsilon}} \operatorname{com} \sigma_{\varepsilon}(0)=u \mathrm{e} \sigma(\mathbb{R}) \subset \mathcal{A}_{\pi_{\varepsilon}}$. Como $\mathcal{A}_{\pi_{\varepsilon}}$ é um conjunto compacto, existe uma sequência $\left(t_{n}\right)_{n \in \mathbb{N}}$ em $(0, \infty)$ e uma função $v \in H^{1}(\Omega)$ tal que $\iota_{n} \rightarrow \infty$ e $\sigma_{\varepsilon}\left(-l_{n}\right) \rightarrow v$ quando $n \rightarrow \infty$. Pelo Teorema 3.2 de [16] temos que $v$ é um ponto de equilíbrio de $\pi_{\varepsilon}$, e como $\mathcal{L}_{r}$ é não crescente ao longo de $\sigma_{\varepsilon}$ e contínua, temos que $\mathcal{L}_{\dot{\varepsilon}}(u) \leq \mathcal{L}_{\bar{\varepsilon}}(v)$. Assim, utilizando as desigualdades (5.10) e (5.12) temos que

$$
\begin{aligned}
& \mathcal{L}_{\varepsilon}(u) \leq \frac{1}{2} \dot{u}_{\varepsilon}(v, v)-\int_{\Omega} \hat{F}(v) \mathrm{d} x \mathrm{~d} y \leq \frac{1}{\eta} a_{\varepsilon}(v, v)+\int_{\Omega}\{\hat{F}(v) \mid \mathrm{d} x \mathrm{~d} y \\
& \leq \frac{1}{2} a_{\varepsilon}(v, v)+\sup _{|v|_{H 1} 1_{1} \leq s, l v / k}|\hat{F}(v)|_{L^{1}(\Omega)}=\frac{1}{2} a_{\xi}(v, v)+\theta \\
& \leq \frac{1}{2}\left((-\xi+\eta)|\forall|_{f^{2}\{(\leq 2\}}^{2}+c\right)+\theta \leq \frac{1}{2}((-\xi+\eta) c: / \cdot+c)+\theta=K^{\prime} .
\end{aligned}
$$

A desigualdade (5.8) implica que

$$
|u|_{\Sigma}^{2} \leq \frac{1}{h_{i}}\left(K^{\prime}+c\right) \text {, para todo } u \in \mathcal{A}_{\pi_{\Sigma}} \text { e para todo } \varepsilon \in(0,1] .
$$

Para o caso $\varepsilon=0$, a demonstração é análoga. Nesse caso, $v \in H_{s}^{1}(\Omega)$ e utilizamos as desigualdades (5.11) e (5.9) no lugar de (5.10) e (5.8). A demonstraçâo está terminada. $\square$

Podemos agora apresentar o resultado de semicontinuidade superior em $\varepsilon=0$ da família de atratores $\left(\mathcal{A}_{\pi_{s}}\right)_{\varepsilon \in[0,1]}$.

Teorema 5.2.2 A familia de atratores $\left(\mathcal{A}_{\pi_{e}}\right)_{\varepsilon \in[0,1]}$ é semiconitínuá superiormente en $\varepsilon=$ 0 com relaçáo à fumilia de normas $|\cdot|_{\varepsilon}$, isto é,

$$
\lim _{\Sigma \rightarrow 0} \sup _{u \in \mathcal{A}_{\pi_{c}}} \inf _{v \in \mathcal{A}_{\pi_{0}}} \mid u-u=0
$$


Demonstração. Vamos provar o resultado por contradição. Suponhamos que existam $\delta>0$, uma sequência $\left(\varepsilon_{n}\right)_{n \in \mathbb{N}}$ em $(0,1], \operatorname{com} \varepsilon_{n} \rightarrow 0^{+}$quando $n \rightarrow \infty$, e, para cada $n \in \mathbb{N}$, uma solução limitada $\sigma_{n}$ de $\pi_{\varepsilon_{n}}$ tais que,

$$
\inf _{v \in \mathcal{A}_{\pi_{0}}}\left|u_{n}-v\right|_{z_{n}}>\delta \text { para todo } n \in \mathbb{N}
$$

onde $u_{n}:=\sigma_{n}(0)$

Pelo Lema 5.2.1 segue que

$$
\sup _{t \in \mathbb{R}}\left|\sigma_{n}(t)\right|_{-n}^{2} \leq \frac{1}{k}\left(K^{\prime}+c\right) \text { para todo } n \in \mathbb{N} .
$$

O Corolário 4.2.4 implica que existe uma subsequência de $\left(\sigma_{n}\right)_{n \in \mathbb{N}}$, denotada novamente por $\left(\sigma_{n}\right)_{n \in \mathbb{N}}$, e uma solução completa $\sigma$ de $\pi_{0}$ tal que

$$
\left|\sigma_{n}(l)-\sigma(l)\right|_{\varepsilon_{n}} \rightarrow 0 \text { para todo } l \in \mathbb{R} .
$$

Segue de (5.14) e (5.15) que

$$
\sup _{t \in \mathbb{R}}|\sigma(t)|_{H^{1}(\Omega)}^{2} \leq \frac{1}{k}\left(K^{\prime}+c\right) .
$$

Logo, $\sigma(\mathbb{R}) \subset \mathcal{A}_{\pi_{0}}$. Fazendo $t=0 \mathrm{em}(5.15)$ temos que

$$
\left|u_{n}-v\right|_{\varepsilon_{n}} \rightarrow 0 \text { quando } n \rightarrow \infty,
$$

onde $v:=\sigma(0) \in \mathcal{A}_{\pi_{0}}$, contradizendo (5.13). 


\section{Capítulo 6}

\section{Domínios bem Decompostos}

Em [20], os autores introduziram a classe dos chamados domínios bem decompostos. Eles mostraram que para essa classe de domínios, tanto o operador $A_{0}$ quanto seu domínio de definição podem ser explicitamente caracterizarlos. Neste capítulo apresentarcmos a definição destes domínios e o resultado de [20] que caracteriza o problema limite. Na Seção 6.1 combinamos resultados de [20] e [18] c caracterizamos os espaços $1 l_{s}^{1}(\Omega)$ e $L_{s}^{2}(\Omega)$ no caso em que $\Omega$ é um domínio com seções verticais conexas. Com estes resultados caracterizamos os espaços $H_{s}^{1}(\Omega)$ e $L_{s}^{2}(\Omega)$ no caso em que $\Omega$ é um domínio bem decomposto. Isso é realizado na Seção 6.2. Finalizamos com um exemplo de domínio bem decomposto e uma análise dos autovalores de $A_{0}$ por meio de resultados de [4].

\subsection{Domínios com seções verticais conexas}

Dizemos que um conjunto aberto $\Omega \subset \mathbb{R}^{M+N}$ possui seções verticais conexas se, para todo $x \in \mathbb{R}^{M}$, a seção vertical $\Omega_{x}:=\left\{y \in \mathbb{R}^{N} \mid(x, y) \in \Omega\right\}$ é conexa. Denotaremos por $P: \mathbb{R}^{M+N} \longrightarrow \mathbb{R}^{M}$ a projeção na primeira coordenarla, isto é, $P(x, y):=x$. Vemos que $\Omega_{x}$ é não-vazio se, e somente se, $x \in P(\Omega)$. Neste caso, definimos $J:=P(\Omega)$ e a função $p: J \longrightarrow(0, \infty)$ por $p(x):=\mu_{N}\left(\Omega_{x}\right)$, onde $\mu_{N}$ é a medida de Lebesgue $N$-dimensional. Observemos que o conceito de seçôes verticais conexas já apareceu na Seção 3.2 .

A próxima proposição, demonstrada em [20] caracteriza as funções $u \in L^{2}(\Omega)$ tais que $\nabla_{y} u=0$ para o caso em que $\Omega$ possui seções verticais conexas.

Proposição 6.1.1 Suponhamos que $\Omega$ possua seções verticais conexas. Seja $u \in L^{2}(\Omega)$ satifazendo $\nabla_{y} u=0$ no sentido das distribuições.

(1) Existe um conjunto de medida nula $S \subset \mathbb{R}^{M+N}$ e uma funçâo $v \in L_{\mathrm{loc}}^{\mathrm{l}}(J)$ tal que $u(x, y)=v(x)$, para todo $(x, y) \in \Omega \backslash S$. Além disso, $p^{1 / 2} v \in L^{2}(J)$.

(2) Se $u \in H^{1}(\Omega)$, então $\partial_{x_{i}} v \in L_{\mathrm{loc}}^{1}(J)$ para $i=1, \ldots, M$. Mais ainda, podemos escolher o conjunto $S$ de $(1)$ de forma que $u(x, y)=v(x)$ e $\partial_{x_{i}} u(x, y)=\partial_{x_{i}} v(x)$ 
para todo $(x, y) \in \Omega \backslash S$ e $i=1, \ldots, M$. Além disso, $p^{1 / 2} \partial_{x_{i}} v \in L^{2}(J), i=1, \ldots, M$.

(3) Se $M=1$, então a função $v$ pode ser escolhida como sendo absolutamente contínua em J. Neste caso, a função $\tilde{u}: \Omega \longrightarrow \mathbb{R}$ dada por $\tilde{u}(x, y):=v(x)$ é um representante contínuo de u.

Demonstração. Como no Teorema 2.2.4, podemos cobrir $\Omega$ com uma família enumerável $\left(\Omega_{n}\right)_{n \in \mathbb{N}}$, com $\Omega_{n}=U_{n} \times V_{n}$, onde $U_{n}$ e $V_{n}$ são retângulos abertos limitados. Obscrvemos que

$$
\nabla_{y}\left(\left.u\right|_{\Omega_{n}}\right)=\left.\left(\nabla_{y} u\right)\right|_{\Omega_{n}}=0 \text { para todo } n \in \mathbb{N}
$$

Logo, como no Teorema 2.2.4, para cada $n \in \mathbb{N}$, existe um conjunto de medida nula $S_{n} \subset \mathbb{R}^{M+N}$ e uma função $v_{n} \in L_{\text {loc }}^{1}\left(U_{n}\right)$ tal que $u(x, y)=v_{n}(x)$, para todo $(x, y) \in \Omega_{n} \backslash S_{n}$. Definimos $S:=\bigcup_{n=1}^{\infty} S_{n}$. Notemos que

$$
\mu_{M+N}(S)=\int_{\mathbb{R}^{M}} \mu_{N}\left(S_{x}\right) \mathrm{d} x=0 .
$$

Assim, existe um conjunto de medida nula $Z \subset \mathbb{R}^{M}$ tal que, para todo $x \in \mathbb{R}^{M} \backslash Z$, $\mu_{N}\left(S_{x}\right)=0$. Alćm disso, podcmos supor que $J=\prod_{-i=1}^{\mu} U_{n}$. Agora, se $x \in J \backslash Z$, então existe um $y \in \Omega_{x} \backslash S_{x}$ tal que, para todo $m \in \mathbb{N} \operatorname{com} x \in U_{m}$, temos que $u(x, y)=v_{m}(x)$. Em particular

$$
\left.v_{m}\right|_{\left(U_{n} \cap U_{n}\right) \backslash Z}=\left.v_{n}\right|_{\left(U_{m} \cap U_{n}\right) \backslash Z} \text { quaisquer que sejam } m, n \in \mathbb{N} .
$$

Consequentemente, existe uma única função $v: J \backslash Z \longrightarrow \mathbb{R}$ com $v(x)=v_{n}(x)$ para todo $n \in \mathbb{N}$ e para todo $x \in U_{n} \backslash Z$. Afirmamos que estendendo $v$ trivialmente a $J$, isto é, definindo $v(x)=0$ se $x \in Z$, obtemos uma função $v \in L_{\text {loc }}^{1}(J)$ tal que $u(x, y)=v(x)$ para todo $(x, y) \in \Omega \backslash S$. De fato, suponhamos que $A$ seja um conjunto mensurável tal que $A \subset \subset J$. Então existem $U_{k_{1}}, \ldots, U_{k_{j}}$ de forma que $A \subset \bigcup_{i=1}^{j} U_{k_{i}}$ e ainda $A \cap U_{k_{2}} \subset \subset U_{k_{i}}$. Logo,

$$
\int_{A}|v(x)| \mathrm{d} x \leq \sum_{i=1}^{j} \int_{A \cap U_{k_{i}}}\left|v_{k_{i}}(x)\right| \mathrm{d} x<\infty
$$

c a demonstração de nossa afirmativa está completa. Como $u \in L^{2}(\Omega)$, temos que

$$
\int_{\Omega} u^{2} \mathrm{~d} x \mathrm{~d} y=\int_{J} p v^{2} \mathrm{~d} x=\int_{J}\left(p^{1 / 2} v\right)^{2} \mathrm{~d} x
$$

e, assim, $p^{i / z} v \in I^{2}(J)$. A demonstração de (1) está concluída.

Mostremos (2). Seja $u \in H^{1}(\Omega)$. O Lema 2.2.2 implica que podemos tomar um conjunto de medida nula $S$ de forma que, para todo $m \in \mathbb{N}, v_{n} \in L_{\text {loc }}^{1}\left(U_{m}\right), u(x, y)=$ $v_{m}(x)$ e $\partial_{x_{i}} u(x, y)=\partial_{x_{i}} v_{m}(x)$ para todo $i=1, \ldots, M$ e todo $(x, y) \in \Omega_{m} \backslash S$. Agora observemos que, para cada $i=1, \ldots, M$, existe um conjunto de medida nula $Z_{2}$ e uma função $u_{i}: J \backslash Z_{\imath} \rightarrow \mathbb{R}$ tal que $\partial_{x_{i}} v_{n}(x)=u_{i}(x)$, para todo $n \in \mathbb{N}$ e todo $x \in U_{n}$. 
Tomando $Z:=\bigcup_{i=1}^{M} Z_{i}$ e estendendo trivialmente cada $w_{i}$ a $J$, teremos que $\partial_{x_{i}} v=w_{i}$ e (2) segue.

Suponhamos que $M=1$. O Lema 2.2 .2 implica que cada $v_{m}$ ć absolutamente contínua em $U_{m}$, para cada $m \in \mathbb{N}$. Sabemos que $v_{m}(x)=v_{n}(x)$ sempre que $x \in U_{n} \cap U_{m}$ (ver a demonstração da Proposição 2.2.3). Sendo assim, a extensão $v$ de todas as funções $v_{m}$ é determinada de modo único c é absolutamente contínua em $J$.

No restante dessa seção vamos supor que $\Omega \subset \mathbb{R}^{M+N}$ seja um aberto limitado com sç̧õcs verticais conexas e que $1 / p \in L^{1}(J)$. Apresentamos alguns resultados auxiliares.

Lema 6.1.2 A função $p: J \longrightarrow \mathbb{R}$ é tal que $p \in L^{\infty}(J)$ e $p(x)>0$ para quase todo $x \in J$.

Demonstração. Como $\Omega$ é limitado, temos que $p$ é limitada em $J$, isto é, $p \in L^{\infty}(J)$. Suponhamos agora que exista um conjunto aberto $A \subset J \operatorname{com} \mu_{N}(A)>0$ e tal que $p(x)=0$ para todo $x \in A$. Porém isso contradiz o fato de $\int_{A}^{n} 1 / p(x) \mathrm{d} x=\infty$.

Lema 6.1.3 Suponhamos que $v: J \longrightarrow \mathbb{R}$ seja uma função tal que $p^{1 / 2} v \in L^{2}(J)$. Dado $\delta>0$ existe um $\psi \in \mathcal{D}(J)$ tal que

$$
\int_{.} p(v-\psi)^{2} \mathrm{~d} x<\delta
$$

Demonstração. Seja $\delta>0$ arbitrário. Como $p^{1 / 2} v \in L^{2}(J)$, existe um $\phi \in \mathcal{D}(J)$ tal que

$$
\left|p^{1 / 2} v-\phi\right|_{L^{2}(J)}<\sqrt{\delta} / 2 \text {. }
$$

Notemos que

$$
\int_{J}\left|p^{1 / 2} v-\phi\right|^{2} \mathrm{~d} x=\int_{J} p\left|v-p^{1 / 2} \phi\right|^{2} \mathrm{~d} x=\int_{J \Sigma}\left|v-p^{1 / 2} \phi\right|^{2} \mathrm{~d} x \mathrm{~d} y
$$

Assim

$$
\mid v-p^{1 / 2} \dot{\varphi} L_{L^{2}(\Omega)}<\sqrt{\delta} / 2
$$

Observemos ainda que

$$
\int_{\Omega}\left(p^{-1 / 2} \phi\right)^{2} \mathrm{~d} x \mathrm{~d} y=\int_{\Omega} p^{-1} \phi^{2} \mathrm{~d} x \mathrm{~d} y=\int_{J} \phi^{2} \mathrm{~d} x .
$$

Portanto, $p^{-1 / 2} \phi \in L^{2}(\Omega)$. Como $\phi \in L^{\infty}(J)$ e $p^{-1} \in L^{1}(J)$, temos também que

$$
\int_{J}\left(p^{-1 / 2} \phi\right)^{2} \mathrm{~d} x=\int_{J} p^{-1} \phi^{2} \mathrm{~d} x<\left|\phi^{2}\right|_{L^{\infty}(J)} \int_{J} p^{-1} \mathrm{~d} x
$$

ou seja, $p^{-1 / 2} \phi \in L^{2}(J)$. Logo, existe uma função $\psi \in \mathcal{D}(J)$ tal que

$$
\left|p^{-1 / 2} \phi-\psi\right|_{L^{2}(J)}<\sqrt{\delta} /(2 \sqrt{k}),
$$


onde $k:=\sup _{x \in J} p(x)>0$. Por outro lado,

$$
\begin{aligned}
\left|p^{-1 / 2} \phi-\psi\right|_{L^{2}(\Omega)}^{2} & =\int_{J_{\Omega}}\left|p^{-1 / 2} \phi-\psi\right|^{2} \mathrm{~d} x \mathrm{~d} y=\int_{J_{J}} p\left|p^{-1 / 2} \phi-\psi\right|^{2} \mathrm{~d} x \\
& \leq k \int_{J}\left|p^{-1 / 2} \phi-\psi\right|^{2} \mathrm{~d} x=k\left|p^{-1 / 2} \phi-\psi\right|_{L^{2}(J)}^{2} .
\end{aligned}
$$

Temos

$$
\left|p^{-1 / 2} \phi-\psi\right|_{L^{2}(\Omega)} \leq \sqrt{k}\left|p^{-1 / 2} \phi-\psi\right|_{L^{2}(J)}
$$

Como

$$
|v-\psi|_{L^{2}(\Omega)} \leq\left|v-p^{-1 / 2} \phi\right|_{L^{2}(\Omega)}+\left|p^{-1 / 2} \phi-\psi\right|_{L^{2}(\Omega)} .
$$

utilizando $(6.1),(6.2)$ e $(6.3)$ obtcmos $i v-\left.\psi\right|_{L^{2}(\Omega)} ^{2}<\sqrt{\delta}$. Mas

$$
|v-\psi|_{L^{2}(\Omega)}^{2}=\int_{\Omega}|v-\psi|^{2} \mathrm{~d} x \mathrm{~d} y=\int_{J} p\left|v-\psi^{2}\right|^{2} \mathrm{~d} x .
$$

() lema está demonstrado.

Nossas hipóteses sobre $p$ e sobre $\Omega$ implicam a seguinte caractcrização do espaço $L_{i s}^{2}(\Omega)$ :

Proposição 6.1.4 Se $\Omega$ possui seções verticais conexas, então

$$
L_{s}^{2}(\Omega)=\left\{u \in L^{2}(\Omega) \mid \nabla_{y} u=0\right\} .
$$

Demonstração. Seja $u \in L_{s}^{2}(\Omega)$ e tomemos uma sequência $\left(u_{n}\right)_{n \in \mathbb{N}}$ em $H_{s}^{1}(\Omega)$ tal que $\left|u_{n}-u\right|_{L^{2}(\Omega)} \rightarrow 0$ quando $n \rightarrow \infty$. Segue que, para qualquer que seja $\phi \in \mathcal{D}(\Omega)$ e para cada $i=1, \ldots, N$,

$$
\begin{aligned}
\int_{\Omega} u \partial_{y_{i}} \phi \mathrm{d} \cdot x \mathrm{~d} y & =\int_{\Omega 2}^{n}\left(u-u_{n}+u_{n}\right) \partial_{y_{i}} \phi \mathrm{d} x \mathrm{~d} y \\
& =\int_{\Omega 2}^{n}\left(u-u_{n}\right) \partial_{y_{i}} \phi \mathrm{d} x \mathrm{~d} y+\int_{\Omega_{2}} u_{n} \partial_{y_{i}} \phi \mathrm{d} x \mathrm{~d} y \\
& =\int_{\Omega}^{n}\left(u-u_{n}\right) \partial_{y_{i}} \phi \mathrm{d} x \mathrm{~d} y .
\end{aligned}
$$

A Desigualdade de Hölder implica que

$$
\int_{I_{\Omega}} u \partial_{y_{\imath}} \phi \mathrm{d} x \mathrm{~d} y \leq\left|u-u_{n}\right|_{L^{2}(\Omega)}\left|\partial_{y_{i}} \phi\right|_{L^{2}(\Omega)} \rightarrow \infty \text { quando } n \rightarrow \infty
$$

Logo, $\nabla_{y} u=0$.

Por outro lado, suponhamos que $u \in L^{2}(\Omega)$ satisfaz $\nabla_{y} u=0$ e seja $\delta>0$ arbitrário. A Proposição 6.1 .1 implica que existe um $v \in L_{\text {loc }}^{1}(J) \operatorname{com} p^{1 / 2} v \in L^{2}(J)$ tal que $u(x, y)=$ $v(x)$ para quase todo $(x, y) \in \Omega$. Polo Loma 6.1 .3 , existe um $\psi \in \mathcal{D}(J)$ tal que 


$$
\int_{J} p(v-\psi)^{2} \mathrm{~d} x<\delta
$$

ou seja,

$$
\int_{i s}(v-\psi)^{2} \mathrm{~d} x \mathrm{~d} y<\delta
$$

Definimos $\Phi: \Omega \longrightarrow \mathbb{R}$ por $\Phi(x, y):=\psi(x)$. Segue que $\Phi \in H^{1}(\Omega)$ e que $\overline{\mathrm{V}}_{z}, \Phi=0$, isto é, $\Phi \in H_{s}^{1}(\Omega)$. Além disso,

$$
\int_{\Omega}(u-\Phi)^{2} \mathrm{~d} x \mathrm{~d} y=\int_{\Omega}(v-\psi)^{2} \mathrm{~d} x \mathrm{~d} y<\delta
$$

Como $\delta>0$ e $\Phi \in H_{s}^{1}(\Omega)$, temos que $u \in L_{s}^{2}(\Omega)$. O lema está demonstrado.

O Lema 6.1.2 e a Proposição 6.1.1 implicam que os espaços vetoriais

$$
H_{p}:=\left\{u \in L_{\mathrm{loc}}^{1}(J) \mid p^{1 / 2} u \in L^{2}(J)\right\}
$$

e

$$
V_{p}:=\left\{u \in H_{p} \mid \partial_{x_{i}} u \in L_{\mathrm{loc}}^{1}(J), p^{1 / 2} \partial_{x_{i}} u \in L^{2}(J), i=1, \ldots, M\right\}
$$

estão bem definidos. Definimos em $H_{p}$ e $V_{p}$ os seguintes produtos:

$$
\langle u, v\rangle_{H_{n}}:=\int_{i_{J}} p(x) u(x) v(x) \mathrm{d} x, \text { para } u, v \in H_{p}
$$

$$
\langle u, v\rangle_{v_{p}}:=\int_{. I}^{l} p(x) \nabla u(x) \cdot \nabla v(x) \mathrm{d} x+\int_{I} p(x) u(x) v(x) \mathrm{d} x, \text { para } u, v \in V_{p} .
$$

Proposição 6.1.5 Os produtos $\langle\cdot, \cdot\rangle_{H_{\nu}}$ e $\langle\cdot, \cdot\rangle_{V_{p}}$ definem uma estrutura de espaço de Iilbert em $H_{p}$ e em $V_{p}$ respectivamente.

Demonstração. Observemos que, qualquer que seja $u \in H_{p}$,

$$
\int_{\Omega}|u(x)|^{2} \mathrm{~d} x \mathrm{~d} y=\int_{J} p(x)|u(x)|^{2} \mathrm{~d} x=\int_{J}\left|p^{1 / 2}(x) u(x)\right|^{2} \mathrm{~d} x<\infty
$$

isto é, $u \in L^{2}(\Omega)$. Além disso, para $u, v \in H_{p}$

$$
\langle u, v\rangle_{H_{p}}=\int_{J} p(x) u(x) v(x) \mathrm{d} x=\int_{\Omega} u(x) v(x) \mathrm{d} x \mathrm{~d} y=\langle u, v\rangle_{L^{2}(\Omega)}
$$

Com isso, $H_{p} \subset L^{2}(\Omega)$ e $\langle\cdot, \cdot\rangle_{H_{p}}$ é a restrição de $\langle\cdot, \cdot\rangle_{L^{2}(\Omega)}$ a $H_{p} \times H_{p}$. A Proposição 6.1.4 implica que $H_{p} \subset L_{s}^{2}(\Omega)$. Para mostrarmos que $H_{p}$ munido com a norma induzida por $\langle\cdot,\rangle_{H_{p}}$ é completo, basta mostrarmos que $H_{p}$ é fechado em $L^{2}(\Omega)$. Seja $\left(u_{n}\right)_{n \in \mathbb{N}}$ uma sequência em $I_{p}$ e $u \in L^{2}(\Omega)$ tal que $\left|u_{n}-u\right|_{n_{p}} \rightarrow 0$ quando $n \rightarrow \infty$. Temos que $\left(u_{n}\right)_{n \in \mathbb{N}}$ é uma sequência em $L_{s}^{2}(\Omega)$ e que $L_{s}^{2}(\Omega)$ é um subespaço fechado de $L^{2}(\Omega)$. Logo, $u \in L_{s}^{2}(\Omega)$. 
A Proposição 6.1.4 implica que $\nabla_{y} u=0$. Sendo assim, a Proposição 6.1.1 implica que existe um conjunto de medida nula $S$ em uma função $v \in L_{\text {loc }}^{1}(J)$ tal que $u(x, y)=v(x)$ para todo $(x, y) \in \Omega \backslash S$ e $p^{1 / 2} v \in L^{2}(J)$. Como

$$
\int_{J} p^{1 / 2} v \mathrm{~d} x-\int_{J} p^{1 / 2} u \mathrm{~d} x
$$

segue que $p^{1 / 2} u \in L^{2}(J)$, isto é, $u \in H_{p}$

No caso do espaço $V_{p}$, notemos que se $u \in V_{p}$, então $u \in L^{2}(\Omega), \dot{d}_{x_{i}} u \in L^{2}(\Omega)$ para $i=1, \ldots, M$ e $\nabla_{y} u=0$, ou seja, $u \in H_{s}^{1}(\Omega) \subset H^{1}(\Omega)$. Observemos também que

$$
\langle u, v\rangle_{V_{P}}=\langle u, v\rangle_{I^{1}(\Omega)} \text { quaisquer que sejam } u, v \in V_{p} .
$$

Como $I_{s}(\Omega)$ é fechado, um raciocínio análogo ao do caso do espaço $H_{p}$, implica que $V_{p}$ é também um espaço de Hilbert.

Definimos a aplicação $\imath: L_{s}^{2}(\Omega) \longrightarrow H_{p}$ por $\imath(u):=v$, onde $v$ é a função dada pela Proposição 6.1.1. Segue das Proposiçôes 6.1.1 e 6.1.4 que $\iota$ está bem definida.

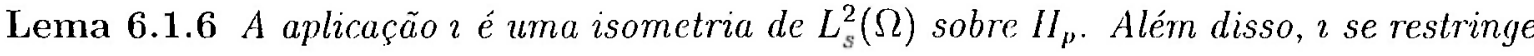
a uma isometria de $I I_{s}^{1}(\Omega)$ sobre $V_{p}$. Assim. $V_{p}$ é denso èm $I I_{p}$.

Demonstração. Sejam $u \in L_{s}^{2}(\Omega)$ e $v=\imath(u)$. Temos que

$$
|v|_{H_{p}}=\int_{J} p(x)|v(x)|^{2} \mathrm{~d} x=\int_{J_{\Omega}}|v(x)|^{2} \mathrm{~d} x \mathrm{~d} y=\int_{J_{\Omega}}|u(x, y)|^{2} \mathrm{~d} x \mathrm{~d} y=|u|_{L^{2}(\Omega)},
$$

ou seja, $\imath$ é uma isometria.

Por outro lado, se $v \in H_{p}$, definimos $u: \Omega \longrightarrow \mathbb{R}$ com $u(x, y):=v(x)$, para todo $(x, y) \in \Omega$. Daí $u \in L^{2}(\Omega)$ satisfaz $\nabla_{y} u=0$. A Proposição 6.1.4 implica que $u \in L_{s}^{2}(\Omega)$. Além disso, $\imath(u)=v$ e portanto $\imath$ é uma aplicação sobrejetora.

Seja $u \in H_{s}^{1}(\Omega)$. Então a Proposição 6.1.1 (2) implica que $\imath(u)=v$, onde $v \in V_{p}$ Notemos que

$$
\begin{aligned}
|v|_{V_{\vartheta}}^{2} & =\sum_{i=1}^{M} \int_{J_{J}} p(x)\left|\partial_{x_{i}} v(x)\right|^{2} \mathrm{~d} x+\int_{J} p(x)|v(x)|^{2} \mathrm{~d} x \\
& =\sum_{i=1}^{M} \int_{J_{s}}\left|\partial_{x_{i}} u(x, y)\right|^{2} \mathrm{~d} x \mathrm{~d} y+\int_{J_{\Omega}}|u(x, y)|^{2} \mathrm{~d} x \mathrm{~d} y=|u|_{H^{1}(\Omega)}^{2} .
\end{aligned}
$$

Portanto,, restrita a $H_{s}^{1}(\Omega)$ é uma isometria em $V_{p}$. Verifiquemos que essa restrição é sobrejetora. Isso segue do fato de que, se $v \in V_{p}$, então $u \in H_{s}^{1}(\Omega)$, onde $u: \Omega \longrightarrow \mathbb{R}$ é dada por $u(x, y):=v(x)$. Isso conclui a demonstração do lema.

Vamos agora assumir que $M=1$ e assim, $J=(a, b)$ para alguns $a, b \in \mathbb{R}$. Nessas condições vamos mostrar que $V_{p} \hookrightarrow H_{p}$ com imersão compacta. Para demonstrar esse resultado necessitamos de uma lema técnico. 
Lema 6.1.7 Seja $u \in V_{p}$ e suponhamos que exista uma função $v \in C^{0}(a, b)$ tal que $u=v$ quase sempre em $(a, b)$. Então $v$ pode ser estendida a uma função contínua em $[a, b]$.

Demonstração. Sejam $x, x^{\prime} \in(a, b) \operatorname{com} x<x^{\prime}$. Então

$$
\left|v(x)-v\left(x^{\prime}\right)\right|=\left|\int_{x}^{x^{\prime}} p^{-1 / 2}(s) p^{1 / 2}(s) v^{\prime}(s) \mathrm{d} s\right| \leq \int_{j_{x}}^{x}\left|p^{-1 / 2} p^{1 / 2} v^{\prime}(s)\right| \mathrm{d} s .
$$

Aplicando a Desigualdade de Hölder obtemos

$$
\begin{aligned}
\left|v(x)-v\left(x^{\prime}\right)\right| & \leq\left(\int_{x}^{x^{\prime}} p^{-1}(s) \mathrm{d} s\right)^{1 / 2}\left(\int_{x}^{x^{\prime}} p(s)\left|v^{\prime}(s)\right|^{2} \mathrm{~d} s\right)^{1 / 2} \\
& \leq|v|_{V_{p}}\left(\int_{\tilde{x}}^{x^{\prime}} p^{-1}(s) \mathrm{d} s\right)^{1 / 2} .
\end{aligned}
$$

Como $1 / p \in L^{1}(a, b)$, temos que $\left|v(x)-v\left(x^{\prime}\right)\right| \rightarrow 0$ quando $x, x^{\prime} \rightarrow a$ (respectivamente $\left.x, x^{\prime} \rightarrow b\right)$. Portanto, existem os limites $v(a):=\lim _{x \rightarrow a} v(x)$ e $v(b):=\lim _{x \rightarrow b} v(x)$. Assim, $v \in C^{0}([a, b])$.

Temos então o seguinte resultado:

Proposição 6.1.8 Suponhamos que $u \in V_{p}$. Então existe uma função $v \in C^{0}([a, b])$ tal que $u=v$ quase sempre em $(a, b)$. Além disso, as imersões $V_{p} \hookrightarrow C^{0}([a, b])$ e $V_{p} \hookrightarrow H_{\eta}$ são compactas.

Demonstração. Seja $\eta>0$ arbitrário tal que $a+\eta<b-\eta$. Afirmamos que existe um $\delta=\delta(\eta)>0$ tal que $p(x) \geq \delta$ para quase todo $x \in(a+\eta, b-\eta)$. De fato, caso contrário, existe um $\eta_{1}>0$ com $a+\eta_{1}<b-\eta_{1}$ de forma que $p(x)<\delta$, para todo $\delta>0$ e todo $x \in\left(a+\eta_{1}, b-\eta_{1}\right)$, contrariando o fato de que $1 / p \in L^{1}(a, b)$. Com isso, observemos que, para todo $u \in V_{p}$,

$$
\int_{J_{a+\eta}}^{\dot{v}-\eta}|u(x)|^{2} \mathrm{~d} x=\int_{J_{a+\eta}}^{b-\eta} p^{-1}(x)\left(p^{1 / 2}(x) u(x)\right)^{2} \mathrm{~d} x \leq \delta \int_{j_{a+\eta}}^{b-\eta}\left(p^{1 / 2}(x) u(x)\right)^{2} \mathrm{~d} x<\infty .
$$

Analogamente,

$$
\int_{a+\eta}^{b-\eta}\left|u^{\prime}(x)\right|^{2} \mathrm{~d} x<\infty
$$

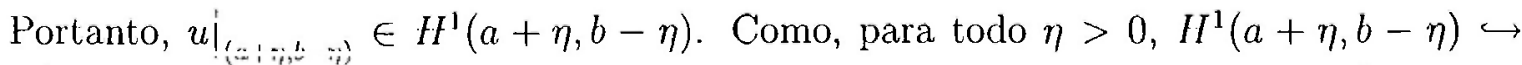
$C^{0}([a+\eta, b-\eta])$ com imersão contínua, temos que existe uma função $v \in C^{0}(a, b)$ tal que $u=v$ quase sempre em $(a, b)$. Pelo Lema 6.1.7,v pode ser cstendida a uma função contínua em $[a, b]$.

Mostremos que as imersões apresentadas no enunciado da proposição são compactas. Seja $u \in V_{p}$ e seja $v$ como na primeira parte dessa proposição 
Fixemos $\eta^{\prime}>0$ com $a+\eta^{\prime}<b-\eta^{\prime}$ e seja $\delta^{\prime}>0$ tal que $p(x) \geq \delta^{\prime}$ para quase todo $x \in\left(a+\eta^{\prime}, b-\eta^{\prime}\right)$. Como no caso de $u$, temos que $\left.v\right|_{\left(a+\eta^{\prime}, b-\eta^{\prime}\right)} \in H^{1}\left(a+\eta^{\prime}, b-\eta^{\prime}\right)$. Segue que existe uma constante $K_{1}>0$ tal que $\left.\left|v_{L^{\infty}\left(a+\eta^{\prime}, b-\eta^{\prime}\right)} \leq K_{1}\right| v\right|_{H^{1}\left(a+\eta^{\prime}, b-\eta^{\prime}\right)}$. Por outro lado,

$$
\begin{aligned}
|v|_{H^{1}\left(a+\eta^{\prime}, b-\eta^{\prime}\right)}^{2} & =\int_{a+\eta^{\prime}}^{b-\eta^{\prime}}|v(x)|^{2} \mathrm{~d} x+\int_{a+\eta^{\prime}}^{b-\eta^{\prime}}\left|v^{\prime}(x)\right|^{2} \mathrm{~d} x \\
& =\int_{a+\eta^{\prime}}^{b-\eta^{\prime}} p^{-1}(x)\left|p^{1 / 2}(x) v(x)\right|^{2} \mathrm{~d} x+\int_{J_{a+\eta^{\prime}}}^{j-\eta^{\prime}} p^{-1}(x)\left|p^{1 / 2}(x) v(x)\right|^{2} \mathrm{~d} x \leq \delta^{\prime}|v|_{V_{p}}^{2} .
\end{aligned}
$$

Segue que existe uma constante $K_{2}>0$ tal que $|v|_{L^{\infty}\left(a+\eta^{\prime}, b-\eta^{\prime}\right)} \leq K_{2}|v|_{V_{p}}$.

Agora, observemos que a desigualdade (6.5) na demonstração do Lema 6.1.7 é válida para todo $x \in(a, b)$. Assim, se $x \in(a, b) \backslash\left(a+\eta^{\prime}, b-\eta^{\prime}\right)$ e $x^{\prime} \in\left(a+\eta^{\prime}, b-\eta^{\prime}\right)$ temos que

$$
|v(x)| \leq\left|v(x)-v\left(x^{\prime}\right)\right|+\left|v\left(x^{\prime}\right)\right| \leq|v|_{V_{p}}\left(\int_{x}^{x^{x}} p^{-1}(s) \mathrm{d} s\right)^{1 / 2}+K_{2}|v|_{V_{\nu}} \leq K_{3}|v|_{V_{p}}
$$

para alguma constante $K_{3}>0$. Logo, definindo $K_{4}:=\max \left\{K_{2}, K_{3}\right\}$ temos que

$$
|v|_{L^{\infty}(a, b)} \leq K_{4}|v|_{v_{p}}
$$

ou seja, $V_{p} \hookrightarrow C^{0}([a, b])$ com imcrsão contínua. Mostromos que essa inclusão ó compacta. Seja $F \subset V_{p}$ um subconjunto limitado. Então $F$ é limitado em $C^{0}([a, b])$ por 6.6. Seja $M>0$ tal que $|v|_{L^{\infty}([a, b])} \leq M$, para todo $v \in F$. E claro que, para cada $x \in[a, b]$, o conjunto $\{v(x) \mid v \in F\}$ é limitado em $\mathbb{R}$, isto é, $F$ é pontualmente limitado. Notemos que $F$ é equicontínuo. De fato, fixemos $x \in[a, b]$ e seja $\eta>0$ arbitrário. Logo, existe um $x^{\prime} \in[a, b]$ tal que

$$
\left(\int_{x}^{x^{\prime}} p^{-1}(x) \mathrm{d} x\right)^{1 / 2}<\eta
$$

Portanto, por (6.5) temos que

$$
\left|v(x)-v\left(x^{\prime}\right)\right| \leq M \eta \text { qualquer que seja } v \in F .
$$

Estamos então nas condições do Teorema de Ascoli-Arzelá, que implica que $F$ é um conjunto relativamente compacto em $C^{0}([a, b])$, demonstrando que a imersão $V_{p} \hookrightarrow C^{0}([a, b])$ é compacta.

Para finalizarmos, observemos que, para todo $u \in C^{0}([a, b])$, temos que $u \in H_{p}$ e

$$
|u|_{H_{p}}=\left(\int_{a}^{\dot{v}} p(x)|u(x)|^{2} \mathrm{~d} x\right)^{1 / 2} \leq\left(|p|_{\left.L^{\infty}(a, b)\right)} \mu(a, b)\right)^{1 / 2}|u|_{L^{\infty}([a, b])}=K|u|_{L^{\infty}([a, b])}
$$

onde $K:=\left(|p|_{L^{\infty}(a, b)} \mu(a, b)\right)^{1 / 2}$. Sendo assim, $C^{0}([a, b]) \hookrightarrow H_{p}$ com imersão contínua e, portanto, $V_{p} \hookrightarrow H_{p}$ com imcrsão compacta. A proposição cstá demonstrada. 


\subsection{Domínios bem decompostos}

Nessa seção vamos apresentar uma classe do domínios $\Omega$ contidos cm $\mathbb{R}^{2}$. Iniciamos observando que, se $\Omega$ é um domínio não-vazio e limitado em $\mathbb{R}^{M+N}$ e $P$ é a projeção na primeira coordenada, então $J_{\Omega}:=P(\Omega)$ ć um domínio não-vázio e limitado de $\mathbb{R}^{M}$. Em particular, se $M=1, J_{\Omega}=\left(a_{\Omega}, b_{\Omega}\right)$, onde $-\infty<a_{\Omega}<\bar{b}_{\Omega}<\infty$.

Vamos estabelecer mais algumas notações. Dado $a \in \mathbb{R}$ e $\delta \in(0, \infty)$, definimos:

$$
\begin{gathered}
I_{\delta}(a):=(a-\delta, a+\delta), \\
I_{\delta}^{-}(a):=(a-\delta, a), \\
I_{\delta}^{+}(a):=(a, a+\delta) .
\end{gathered}
$$

Definição 6.2.1 Suponhamos que $M=N=1$ e que $\Omega, \Omega_{1}$ e $\Omega_{2}$ sejam domínios limitados não-vazios em $\mathbb{R}^{M+N}$. Dado $c \in \mathbb{R}$, dizemos que $\Omega_{1}$ se une a $\Omega_{2}$ no ponto $c$ em $\Omega$ se as seguintes propricdades estiverem satisfeitas:

(1) existem númcros reais positivos $\beta:=\beta_{\Omega_{1}, S \Omega_{2}}$ e $\gamma:=\gamma_{\Omega_{1}, S \Omega_{2}}$, com $\beta<\gamma$, tais que $\Omega_{1} \cap \bar{\Omega}_{2}=\{c\} \times[\hat{\sigma}, \gamma]$

(2) $c:=a_{\Omega \Omega_{2}}=b_{\Omega \Omega_{1}}$;

(3) $\{c\} \times(\beta, \gamma) \subset \Omega$;

(4) sempre que $d \in(\beta, \gamma)$, existe um $\delta=\delta(d)>0$ tal que

$$
\begin{gathered}
I_{\delta}(d) \subset(\beta, \gamma), \\
I_{\delta}^{-}(c) \times I_{\delta}(d) \subset \Omega_{1}, \\
I_{\delta}^{+}(c) \times I_{\delta}(d) \subset \Omega_{2} .
\end{gathered}
$$

Dizemos que $\Omega_{1}$ e $\Omega_{2}$ se unem no ponto $\mathrm{c}$ em $\Omega$ se $\Omega_{1}$ se une a $\Omega_{2}$ no ponto $c \mathrm{em} \Omega$ ou se $\Omega_{2}$ se une a $\Omega_{1}$ no ponto $c$ em $\Omega$.

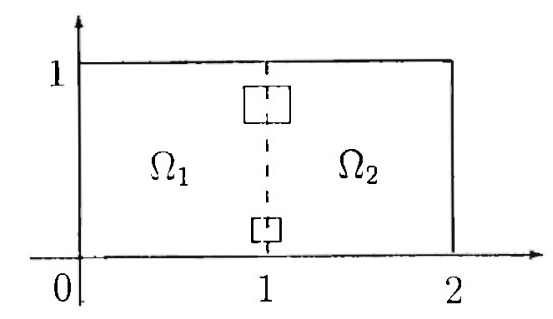

Figura 1: $\Omega_{1}$ so uno a $\Omega_{2}$ no ponto $c=1 \mathrm{em} \mathrm{cm} \Omega=(0,2) \times(0,1)$. 
Exemplo 6.2.2 Sejam $\Omega=(0,2) \times(0,1) \subset \mathbb{R}^{2}, \Omega_{1}=(0,1) \times(0,1) \subset \mathbb{R}^{2}$ e $\Omega_{2}=$ $(1,2) \times(0,1) \subset \mathbb{R}^{2}$. Na Figura 1 encontramos a representação geométrica de $\Omega, \Omega_{1}$ e $\Omega_{2}$. Observemos que $\Omega_{1} \cap \Omega_{2}=\{1\} \times[0,1]$ e $1=a_{\Omega_{3}}-b_{\Omega_{1}}$. Além disso, para todo $d \in(0,1)$, existe um $\delta=\delta(d)>0$ tal que as inclusões da condição (4) da Definição 6.2.1 estão satisfeitas, ou seja, $\Omega_{1}$ se une a $\Omega_{2}$ no ponto $c=1 \mathrm{em} \Omega$.

No restante dessa seção vamos assumir que $M=N=1$. Apresentamos a scguinte definição técnica importante no que segue:

Definição 6.2.3 Seja $\Omega \subset \mathbb{R}^{2}$ um domínio limitado, aberto, não-vazio e com fronteira Lipschitz e seja $P: \mathbb{R}^{2} \longrightarrow \mathbb{R}$ a projeção na primcira coordenada. Una boa decomposição de $\Omega$ é urna coleção $\Omega_{1}, \ldots, \Omega_{r}$ de subconjuntos de $\Omega$, abertos e não-vazios, conexos e dois a dois disjuntos, com seção vertical conexa satisfazendo as seguintes propriedades:

(1) $\Omega \backslash\left(\bigcup_{k=1}^{r} \Omega_{k}\right) \subset Z$, onde $Z:=\bigcup_{k=1}^{r}\left(\left\{a_{\Omega_{k}}, b_{\Omega_{k}}\right\} \times \mathbb{R}\right)$;

(2) para cada $k=1, \ldots, r, \partial \Omega_{k} \subset \partial \Omega \bigcup\left(\left\{a_{\Omega_{k}}, b_{\Omega_{k}}\right\} \times \mathbb{R}\right)$ e para $c \in\left\{a_{\Omega_{k}}, b_{\Omega_{k}}\right\}$, $\partial \Omega_{k} \cap(\{c\} \times \mathbb{R})=\{c\} \times I$, onde $I$ é um intervalo compacto (podendo ser degenerado) em $\mathbb{R}$;

(3) sempre que $k, l=1, \ldots, r, k \neq l$ e $(c, d) \in \Omega_{k} \cap \Omega_{l}$ é um ponto arbitrário, então, ou $\Omega_{k}$ c $\Omega_{l}$ se unem no ponto $c$ em $\Omega$ ou existe um $m \in\{1, \ldots, r\}$ tal que $\Omega_{k}$ e $\check{s}_{m}$ se unem no ponto $c$ em $\Omega$ e $\Omega_{l}$ e $\Omega_{m}$ se unem no ponto $c$ em $\Omega$;

(4) para cada $k=1, \ldots, r$, a função $p_{k}: J_{\Omega_{k}} \longrightarrow(0, \infty)$ definida por $p_{k}(x):=\mu_{1}\left(\left(\Omega_{k}\right)_{x}\right)$ é tal que $1 / p_{k} \in L^{1}\left(J_{\iota_{k}}\right)$.

Dizernos que um domínio $\Omega \subset \mathbb{R}^{2}$ é bem decomposto se existe uma boa decomposição de $\Omega$.

Intuitivamente, a Definição 6.2.3 nos diz que, a menos de un conjunto de medida nula contido em um conjunto $Z$, o qual é união finita de retas verticais, $\Omega$ pode ser decomposto cm uma cuantidade finita de domínios $\Omega_{k}, k=1, \ldots, r$, de maneira que os vários conjuntos $\Omega_{k}$ e $\Omega_{l}$ se "unem" em $Z$ de um modo "bom". Os pontos de $\bar{\Omega} \cap Z$ são aqueles nos quais as componentes concxas das seções verticais $\Omega_{x}$ se bifurcam.

Proposição 6.2.4 Seja $\Omega \subset \mathbb{R}^{2}$ um domínio bem decomposto. Se $\Omega_{1}, \ldots, \Omega_{r}$ é uma boa decomposição de $\Omega$, entâo $\Omega=\bigcup_{k=1}^{r} \Omega_{k}$.

Demonstração. É claro que $\bigcup_{k=1}^{r} \Omega_{k} \subset \Omega$. Seja $z \in \bar{\Omega}$. Então existe uma sequência $\left(z_{n}\right)_{n \in \mathbb{N}}$ em $\Omega$ com $z_{n} \rightarrow z$. Podemos supor que $z_{n} \notin Z$, para todo $n \in \mathbb{N}$. De fato, dado $n \in \mathbb{N}$, se $z_{n} \in Z$ entào existe um $\xi_{n} \in \mathbb{R}^{2} \operatorname{com} z_{n}+\dot{\xi}_{n} \in \Omega \backslash Z$ e $\left|\xi_{n}\right| \leq n^{-1}$. Sc $z_{n} \notin Z$, cntão definimos $\xi_{n}:=0$. Neste caso, temos uma secuência $\left(\xi_{n}\right)_{n \in \mathbb{N}}$ tal que 


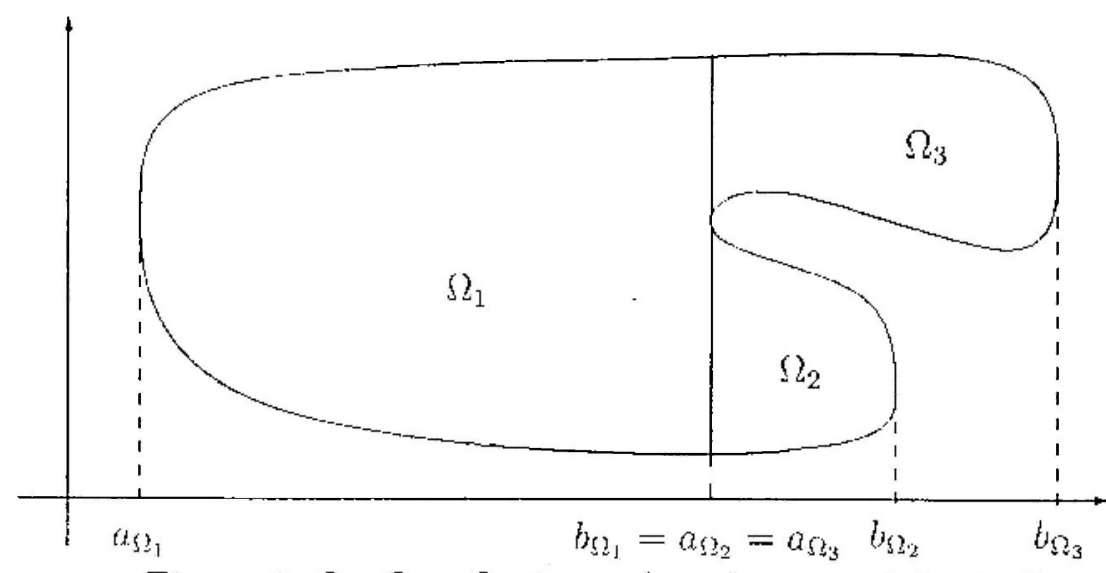

Figura 2: $\Omega_{1}, \Omega_{2}$ e $\Omega_{3}$ o una boa decomposiça de $\Omega$.

$\xi_{n} \rightarrow 0, z_{n}+\xi_{n} \in \Omega, z_{n}+\xi_{n} \notin Z$ e $z_{n}+\xi_{n} \rightarrow z$. Portanto, tomando uma subsequência se necessário, podemos assumir que existe um $l \in\{1, \ldots, r\}$ tal que $z_{n} \in \Omega_{l}$, para todo $n \in \mathbb{N}$ e, assim, $z \in \bar{\Omega}_{l}$.

Exemplo 6.2.5 Consideremos $\Omega \subset \mathbb{R}^{2}$ dado pela Figura 2 e sejam $\Omega_{1}, \Omega_{2}$ e $\Omega_{3}$ subconjuntos em $\mathbb{R}^{2}$ dados pela mesma figura. Temos que $\Omega_{1}, \Omega$, e $\Omega_{3}$ é uma boa decomposição de $\Omega$. De fato, as condięões (1)-(3) da Definição 6.2 .3 são verificadas facilmente. No caso de (4), obscrvemos que, existe um $\delta>0$ tal que $p(x)>0$ para $x$ próximo aos pontos $a_{\Omega_{1}}$, $b_{\Omega_{2}}$ e $b_{\Omega_{3}}$. Portanto $1 / p$ é integrável cm alguma vizinhança desses pontos.

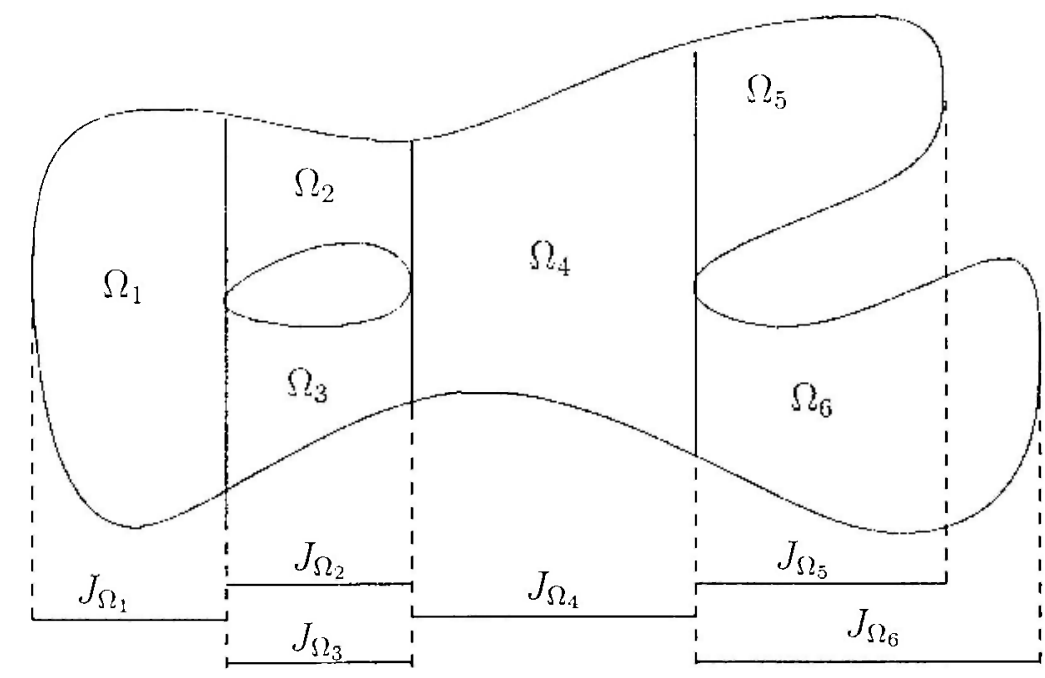

Figura 3: Um exemplo mais geral de domínio bem decomposto.

Na figura 3 apresentamos mais uma representação de um domínio bem decomposto.

O próximo exemplo apresenta um caso específico de domínios bem decompostos. Os domínios en forma de $C$, que serão estudados na Seção 6.3, são casos particulares deste 
exemplo.

Exemplo 6.2.6 Sejam $R_{1}, \ldots, R_{s}$ retângulos fechados e limitados em $\mathbb{R}^{2}$ com interior não-vazio e com lados paralclos aos cixos coordenados. Scja $\Omega_{R}$ o interior de $\bigcup_{k=1}^{s} R_{k}$. Então qualquer componente conexa $\Omega$ de $\Omega_{R}$ é um domínio bem decomposto com fronteira Lipschitz.

Dada uma boa decomposição $\Omega_{1}, \ldots, \Omega_{r}$ de $\Omega$, definimos o conjunto

$$
\left.E:=\bigcup_{k=1}^{r}\left(\left(\left\{a_{\Omega_{k}}, b_{\Omega_{k}}\right\} \times \mathbb{R}\right) \cap \partial \Omega_{k}\right)\right)
$$

O Lema 6.1.7 nos auxiliará a demonstrar o seguinte resultado técnico:

Proposição 6.2.7 Seja $\Omega_{1}, \ldots, \widehat{\Omega} \measuredangle$ uma boa decomposição de $\Omega$. Então toda função $v \in$ $H_{s}^{1}(\Omega) \cap C^{0}(\Omega)$ pode ser estendida de maneira única a uma função contínua $\tilde{v}: \bar{\Omega} \longrightarrow \mathbb{R} . A$ função v̂ é constante em cada componente conexa de $F$. Além disso, para cada componente conexa $Q$ de $E$, existe uma função $v \in H_{s}^{1}(\Omega) \cap C^{0}(\Omega)$ tal que $\tilde{v}_{{ }_{Q}}^{1}=1$ e $\left.\tilde{v}\right|_{\mathscr{L} \backslash Q}=0$.

Demonstração. Seja $v \in H_{s}^{1}(\Omega) \cap C^{0}(\Omega)$ uma função arbitrária. Pela Proposição 6.1.1, para cada $k=1, \ldots, r$, existe uma função absolutamente contínua $v_{k}: J_{\Omega_{k}} \longrightarrow \mathbb{R}$ tal que $v(x, y)=v_{k}(x)$ para todo $(x, y) \in \Omega_{k}$. O Lema 6.1.7 implica que cada $v_{k}$ pode ser estendida continuamente a uma função cm $\left[a_{\Omega_{k}}, b_{\Omega_{k}}\right]$.

Afirmamos que, quaisquer que sejam $k, l=1, \ldots, r$ com $k \neq l$, se $\Omega_{k}$ e $\Omega_{l}$ se unem no ponto $c \in \mathbb{R}$ em $\Omega$, então $v_{k}(c)=v_{l}(c)$. De fato, podemos assumir que

$$
\bar{\Omega}_{k} \cap \Omega_{l}=\{c\} \times[\beta, \gamma],
$$

onde $\beta=\beta_{\Omega_{k}, \Omega_{l}}$ e $\gamma=\gamma_{\Omega_{k} . \Omega_{l}}$ são como na Definição $6.2 .1 \mathrm{e} c=a_{\Omega_{l}}=b_{\Omega_{k}}$. Tomemos $d \in(\beta, \gamma)$ arbitrário e $\delta=\delta(d)$ como na Definição 6.2.1. Como $v(x, y)=v_{k}(x)$ para $x \in I_{\delta}^{-}(c)$ e $v(x, y)=v_{l}(x)$ para $x \in I_{\delta}^{+}(c)$, da continuidade de $v, v_{k}$ e $v_{l}$ segue que $v_{k}(c)=v(c, d)=v_{l}(c)$, provando nossa afirmação.

Como $\bar{\Omega}_{k} \subset\left[a_{\Omega_{k}}, b_{\Omega_{k}}\right] \times \mathbb{R}$, segue que a função $\tilde{v}_{k}: \bar{\Omega}_{k} \rightarrow \mathbb{R}$ dada por $\tilde{v}_{k}(x, y):=v_{k}(x)$ está bem definida e é contínua.

Vamos mostrar agora que para $k, l=1, \ldots, r \operatorname{com} k \neq l$,

$$
\tilde{v}_{k}(c, d)=\tilde{v}_{l}(c, d) \text { para }(c, d) \in \bar{\Omega}_{k} \cap \bar{\Omega}_{l} .
$$

De fato, seja $(c, d) \in \bar{\Omega}_{k} \cap \bar{\Omega}_{l}$ um ponto arbitrário. Então $\Omega_{k}$ e $\widehat{\iota_{l}}$ se unem no ponto $c$ em $\Omega$ ou, caso contrário, existe um $m \in\{1, \ldots, r\}$ tal que $\Omega_{k}$ e $\Omega_{m}$ se unem no ponto $c$ em $\Omega \mathrm{c} \Omega_{t}$ e $\Omega_{m}$ se unem no ponto $c \mathrm{~cm} \Omega$. A afirmação demonstrada acima implica que, no primeiro caso, $v_{k}(c)=v_{l}(c)$ e no segundo, $v_{k}(c)=v_{m}(c)=v_{l}(c)$. Assim, $v_{k}(c)=v_{l}(c)$ e, portanto, $\bar{v}_{k}(c, d)=v_{k}(c)=v_{l}(c)=\tilde{v}_{l}(c, d)$. Portanto, cxiste una única função $\tilde{v}: \bar{\Omega} \longrightarrow \mathbb{R}$ que 
estende todas as funções $\tilde{v}_{b}$. Além disso, $\tilde{v}$ é a única extensão contínua de $v$, o que prova a primeira parte de proposição.

Provemos agora que $\ddot{v}$ ć constante om qualquer componente concxa $Q$ de $E$. A propriedade (2) da Definição 6.2 .3 implica que toda componente conexa $Q$ de $E$ é da forma $Q=\{c\} \times I$, onde $I=[\xi, \eta]$ é um intervalo compacto de $\mathbb{R}$. Tal intervalo é união de um número finito de intervalos $I_{j}$, com $j$ variando $\mathrm{cm}$ um subconjunto de $\{1, \ldots, r\}$, de forma que $c \in\left\{a_{\Omega_{j}}, b_{\Omega_{j}}\right\}$ e $\tilde{v}=\vec{v}_{j}^{\prime}=v(c)$ em $\{c\} \times I_{j}$. Assim, a função contínua $\tilde{v}$ assume um número finito de valores no conjunto conexo $Q$. Como $\tilde{v}(Q)$ é conexo temos que $\hat{v}$ deve ser constante em $Q$.

Passemos à demonstração da última parte da proposição. Seja $Q=\{c\} \times I$ uma componente connexa de $E$.

Afirmamos que existe um $\delta_{1}>0$ tal que o intervalo $J:=\left[\xi-2 \delta_{1}, \eta+2 \delta_{1}\right]$ satisfaz $(\{c\} \times J) \cap \bar{\Omega}=Q$. De fato, suponhamos que a afirmação não seja verdadeira. Então, para cada $n \in \mathbb{N}$, o intervalo $J_{n}:=[\xi-2 / n, \eta+2 / n]$ é tal que $\left(\{c\} \times J_{n}\right) \cap \bar{\Omega} \neq Q$. Portanto, para cada $n \in \mathbb{N}$, existe um $\left(c, y_{n}\right) \in\left(\{c\} \times J_{n}\right) \cap \bar{\Omega} \operatorname{com} y_{n} \notin I$. Como $2 / n \rightarrow 0$ quando $n \rightarrow \infty$, temos que $y_{n} \rightarrow y$ para algum $y \in I$. Pela Proposição 6.2.4, podemos assumir que existe um $l \in\{1, \ldots, r\}$ tal que $\left(c, y_{n}\right) \in \Omega_{l}$, para todo $n \in \mathbb{N}$. Logo, $(c, y) \in \Omega_{l}$.

Por outro lado, a definição do conjunto $E$ implica que existe um $k \in\{1, \ldots, r\}$ tal que $(c, y) \in \partial \Omega_{k} \cap\left(\left\{a_{\Omega_{k}}, b_{\Omega_{k}}\right\} \times \mathbb{R}\right)$. Segue que $(c, y) \in \bar{\Omega}_{k} \cap \Omega_{l}$. Consideremos agora dois casos:

Caso 1: Suponhamos que $k=l$. Então $c \in\left\{a_{S \Omega_{2}}, b_{S_{2}}\right\}$.

Caso 2: Suponhamos $k \neq l$. Entĩo a propriedado (3) da Definição 6.2.3 nos dá novamente que $c \in\left\{a_{\Omega_{l}}, b_{\Omega_{l}}\right\}$.

Consequentemente, $y_{n} \in I_{l}$ para todo $n \in \mathbb{N}$, onde $I_{l}$ é um intervalo tal que $\{c\} \times I_{l}=$ $\partial \Omega_{l} \cap(\{c\} \times \mathbb{R})$. Como $y_{n} \in I_{l}$, a conexidade de $Q$ implica que $I_{l} \subset I$ e, portanto, $y_{n} \in I$ para todo $n \in \mathbb{N}$, o que é uma contradição.

A afirmação demonstrada implica que existe um $\delta_{2}>0$ tal que o conjunto $\left[c-2 \delta_{2}, c+\right.$ $\left.2 \delta_{2}\right] \times\left(\left[\xi-2 \delta_{1}, \xi-\delta_{1}\right] \cup\left[\eta+\delta_{1}, \eta+2 \delta_{1}\right]\right)$ é disjunto de $\Omega$ e $\left[c-2 \dot{\delta}_{2}, c+2 \delta_{2}\right] \times\left[\xi-2 \delta_{1}, \eta+2 \delta_{1}\right]$ é disjunto de $E \backslash Q$. Sejam $\psi: \mathbb{R} \longrightarrow \mathbb{R}$ e $\phi: \mathbb{R} \longrightarrow \mathbb{R}$ funções $C^{\infty}$ tais que $\psi$ possui suporte em $J$ c valc $1 \mathrm{~cm}$ uma vizinhança de $\left[\xi-\hat{o}_{1}, \eta+\delta_{1}\right]$ e $\phi$ possui suporte em $\left[c-2 \delta_{2}, c+2 \delta_{2}\right]$ e vale $1 \mathrm{em}\left[c-\delta_{2}, c+\partial, \bar{j}\right.$. Definimos a função $w: \bar{\Omega} \longrightarrow \mathbb{R}$ por $w(x, y)=\phi(x) \psi(y)$. Segue que $w$ é contínua e que $v:=\left.w\right|_{\Omega}$ pertence a $I^{1}(\Omega) \cap C^{0}(\Omega)$. Também,

$$
\partial_{y} v(x, y)=\phi(x) \psi^{\prime}(y)=0, \text { para todo }(x, y) \in \Omega .
$$

Portanto, $w=\bar{v} \in H^{1}(\Omega) \cap C^{0}(\Omega)$. Observemos que, pela modo que $w$ foi construída, $w=1 \mathrm{em} Q$ e $w=0$ em $E \backslash Q$, o que finaliza a demonstração da última parte da proposição.

O próximo resultado, que é o mais importante desse capítulo, caracteriza o operador $A_{0}$ quando $\Omega \subset \mathbb{R}^{2}$ é um domínio ben decomposto. 
Teorema 6.2.8 Suponhamos que $\Omega \subset \mathbb{R}^{2}$ seja um domínio bem decomposto. Sejam $u: \Omega \longrightarrow \mathbb{R}$ ev: $\Omega \longrightarrow \mathbb{R}$ funções arbitrárias. Então, as seguintes condições são equivalentes:

(1) u pertence ao dominio de definição de $A_{0}$ e $A_{0} u=v$;

(2) existem funções $u_{k}: J_{\Omega_{k}} \longrightarrow \mathbb{R}$ e $v_{k}: J_{\Omega_{k}} \longrightarrow \mathbb{R}, k=1, \ldots, r$, e um conjunto de medida nula $S \subset \mathbb{R}^{2}$, tais que as seguintes propriedades sào verificadas:

(a) para cada $k=1, \ldots, r$, temos que $u_{k} \in V_{p_{k}}$ e $v_{k} \in H_{p_{k}}$. A derivada no sentido das distribuiçöes $\left(p_{k} \cdot u_{k}^{\prime}\right)^{\prime}$ de $p_{k} \cdot u_{k}^{\prime}$ pertence a $L_{\text {loc }}^{1}\left(J_{S \lambda_{k}}\right)$ e $\left(p_{k} \cdot u_{k}^{\prime}\right)^{\prime}=-p_{k} v_{k}$. Além disso, $u(x, y)=u_{k}(x)$ e $v(x, y)=v_{k}(x)$ para todo $(x, y) \in \Omega_{k} \backslash S$;

(b) os limites $u_{k}(c):=\lim _{x \rightarrow c} u_{k}(x)$ e $u_{l}(c):=\lim _{x \rightarrow c} u_{l}(x)$ existem e sâo iguais sempre que $k, l \in\{1, \ldots, r\}$ com $k \neq l$, e $\Omega_{k}$ e e $\Omega_{l}$ se unem no ponto c em $\Omega$;

(c) sempre que $Q$ for uma componente conexa de $E$, a qual é necessariamente da forma $Q=\{c\} \times I$, onde $c \in \bigcup_{k=1}^{r}\left\{a_{\Omega_{k}}, b_{\Omega_{k}}\right\}$ e I é um intervalo, então

$$
\sum_{k \in \sigma_{+}}\left(p_{k} \cdot u_{k}^{\prime}\right)(c)=\sum_{k \in \sigma}\left(p_{k} u_{k}^{\prime}\right)(c)
$$

onde

$$
\begin{aligned}
& \sigma_{+}=\sigma_{+}(Q):=\left\{k \mid \bar{\Omega}_{k} \cap\left(\left\{b_{\Omega_{k}}\right\} \times \mathbb{R}\right) \subset Q, b_{\Omega_{k}}=c\right\} \\
& \sigma_{-}=\sigma_{-}(Q):-=\left\{k \mid \bar{\Omega}_{k} \cap\left(\left\{a_{\Omega_{k}}\right\} \times \mathbb{R}\right) \subset Q, a_{\Omega_{k}}=c\right\}
\end{aligned}
$$

Demonstração. Suponhamos que $u \in D\left(A_{0}\right)$ e que $A_{0} u=v$. Pela Proposição 6.1.1, podemos assumir que ué uma função contínua. Fixcmus $k=1, \ldots, r$ arbitrariamente. Notemos que a mesma proposição implica que existem funções absolutamente contínuas $u_{k} . J_{\Omega_{k}} \rightarrow \mathbb{R}$ e $v_{k}: J_{\Omega_{k}} \rightarrow \mathbb{R}$ e um conjunto de medida nula $S_{k} \subset \mathbb{R}^{2}$ tais que $u(x, y)=$ $u_{k}(x)$ e $v(x, y)=v_{k}(x)$ para todo $(x, y) \in \Omega_{k} \backslash S_{k}$. Além disso, podemos assumir que $u_{k}^{\prime} \in L_{\mathrm{loc}}^{1}\left(J_{\Omega_{k}}\right)$, onde a derivada ć tomada no sentido das distribuições, c ainda $\partial_{x} u(x, y)=$ $u_{k}^{\prime}(x)$ para todo $(x, y) \in \Omega_{k} \backslash S_{k}$. A Proposição 6.1.1 também implica que $p_{k}^{1 / 2} u_{k}, p_{k}^{1 / 2} v_{k}$ e $p_{k}^{1 / 2} u_{k}^{\prime}$ pertencem a $L^{2}\left(J_{\Omega_{k}}\right)$.

Mostremos que $\left(p_{k} \cdot u_{k}^{\prime}\right)^{\prime}=-p_{k} \cdot v_{k}$ no sentido das distribuições. Para isso, tomemos $\phi \in \mathcal{D}\left(J_{\Omega_{k}}\right)$ arbitrária e definamos $L:=\operatorname{supp} \phi \subset J_{\Omega_{k}}$. Queremos mostrar que

$$
\int_{J_{\Omega_{\Omega_{k}}}}\left(p_{k} u_{k}^{\prime}\right)^{\prime} \phi \mathrm{d} x=-\int_{{ }^{\prime} J_{\Omega_{k}}} p_{k} v_{k} \phi \mathrm{d} x .
$$

Definimos uma função $w: \Omega \longrightarrow \mathbb{R}$ por

$$
w(x, y):= \begin{cases}\phi(x), & \text { se }(x, y) \in \Omega_{k} \\ 0, & \text { caso contrário. }\end{cases}
$$


Afirmamos que $w \in H_{s}^{1}(\Omega)$. De fato, observemos que $w$ é de classe $C^{\infty}$ em cada $\Omega_{l}$, $l=1, \ldots, r$. Como tais conjuntos são disjuntos, $w$ é de classe $C^{\infty}$ em $\bigcup_{l=1}^{7} \Omega_{l}$. Seja $(x, y) \in \Omega \backslash\left(\bigcup_{l=1}^{r} \Omega_{l}\right)$ arbitrário. Logo, $(x, y) \in Z$ e, em particular, $x^{\prime} \notin L$, para todo $\left(x^{\prime}, y^{\prime}\right)$ em uma vizinhança de $(x, y)$. Agora, para qualquer que seja $\left(x^{\prime}, y^{\prime}\right)$ dessa forma, segue que $w\left(x^{\prime}, y^{\prime}\right)=0$, isto é, $w$ é nula em uma vizinhança de $(x, y)$ e portanto de classe $C^{\infty}$ nessa vizinhança. Com isso, concluímos que $w \in C^{\infty}(\Omega)$, e assim, $w \in H_{s}^{1}(\Omega)$. Segue que

$$
\begin{aligned}
\int_{\Omega} \nabla u \cdot \nabla w \mathrm{~d} x \mathrm{~d} y & =\int_{\Omega_{k}} \nabla u \cdot \nabla w \mathrm{~d} x \mathrm{~d} y=\int_{J_{\Omega_{k}}} p_{k} \nabla u_{k} \cdot \nabla w \mathrm{~d} x \\
& =\int_{J_{\Omega_{k}}} p_{k} u_{k}^{\prime} \phi^{\prime} \mathrm{d} x=-\int_{J_{\Omega_{k}}}\left(p_{k} \cdot u_{k}^{\prime}\right)^{\prime} \phi \mathrm{d} x .
\end{aligned}
$$

Além disso,

$$
\int_{\Omega} v w \mathrm{~d} x \mathrm{~d} y=\int_{\Omega_{k}} v w \mathrm{~d} x \mathrm{~d} y=\int_{J_{\Omega_{k}}} p_{k} v_{k} \phi \mathrm{d} x
$$

Por outro lado, como $u \in D\left(A_{0}\right)$ e $A_{0} u=v$, temos que

$$
\int_{\Omega}^{r} \nabla u \cdot \nabla \omega \mathrm{d} x \mathrm{~d} y=\int_{\Omega}^{r} v \omega \mathrm{d} x \mathrm{~d} y, \text { qualquer que seja } \omega \in H_{s}^{1}(\Omega),
$$

Em particular

$$
-\int_{J_{\Omega_{k}}}\left(p_{k} \cdot u_{k}^{\prime}\right)^{\prime} \phi \mathrm{d} x=\int_{J_{\Omega_{k}}} p_{k} v_{k} \phi \mathrm{d} x
$$

Isso conclui a demonstração da parte (a).

Pela prova da Proposição 6.2 .7 vemos que, para cada $k=1, \ldots, r$, e para $c \in$ $\left\{a_{\Omega_{k}}, b_{\Omega_{k}}\right\}$, o limite $u_{k}(c):=\lim _{x \rightarrow c} u_{k}(x)$ existe. Se $k \neq l$ e $\Omega_{k}$ se une a $\Omega_{l}$ em algum ponto $c \in \mathbb{R}$ em $\Omega$, então $c=a_{\Omega_{l}}=b_{\Omega_{k}}$. Seja $d \in(\beta, \gamma)$ arbitrário e $\delta=o$ óà) como na Definição 6.2.1. A continuidade de $u$ implica que

$$
\begin{aligned}
u_{k}(c) & =\lim _{x \rightarrow b_{\Omega_{k}}^{-}} u_{k}(x)=\lim _{x \rightarrow b_{\Omega_{k}}^{-}} u(x, d) \\
& =u(c, d)=\lim _{x \rightarrow a_{\Omega_{l}}^{+}} u(x, d)=\lim _{x \rightarrow a_{\Omega_{l}}^{+}} u_{l}(x)=u_{l}(c),
\end{aligned}
$$

provando assim a parte (b).

Seja agora $w \in H_{s}^{1}(\Omega) \cap C^{0}(\Omega)$ arbitrária e $w_{k}, k=1, \ldots, r$, e $\tilde{w}$ como na Proposição 6.2.7. Pcla parte (a) já provada, para cada $k=1, \ldots, r$ e $c \in\left\{a_{\Omega_{k}}, b_{\Omega_{k}}\right\}$, existe o limite

$$
\lim _{x \rightarrow c}\left(p_{k} u_{k}^{\prime}\right)(x)=:\left(p_{k} u_{k}^{\prime}\right)(c)
$$

Além disso, a prova da Proposição 6.2.7 implica que existe o limite

$$
\lim _{x \rightarrow c} w_{k}(x)=: w_{k}(c)
$$


Assim, para cada $k=1 \ldots, r$, temos que

$$
\begin{aligned}
\int_{J_{\Omega_{k}}} p_{k} u_{k}^{\prime} w_{k}^{\prime} \mathrm{d} x & =\left(p_{k} u_{k}^{\prime}\right)\left(b_{\Omega_{k}}\right) w_{k}\left(b_{\Omega_{k}}\right)-\left(p_{k} u_{k}^{\prime}\right)\left(a_{\Omega_{k}}\right) w_{k}\left(a_{\Omega_{k}}\right)-\int_{J_{\Omega_{k}}}\left(p_{k} u_{k}^{\prime}\right)^{\prime} w_{k} \mathrm{~d} x \\
& =\left(p_{k} u u_{k}^{\prime}\right)\left(b_{\Omega_{k}}\right) w_{k}\left(b_{\Omega_{k}}\right)-\left(p_{k} \cdot u_{k}^{\prime}\right)\left(a_{\Omega_{k}}\right) w_{k}\left(a_{\Omega_{\Omega_{k}}}\right)+\int_{J_{\Omega_{\Omega_{k}}}} p_{k} v_{k} w_{k} \mathrm{~d} x
\end{aligned}
$$

Portanto

$$
\sum_{k=1}^{r} \int_{J_{\Omega_{k}}} p_{k} u_{k}^{\prime} w_{k}^{\prime} \mathrm{d} x=\sum_{k=1}^{r}\left(\left(p_{k} u_{k}^{\prime}\right)\left(b_{\Omega_{k}}\right) w_{k}\left(b_{\Omega_{k}}\right)-\left(p_{k} u_{k}^{\prime}\right)\left(a_{\Omega_{k}}\right) w_{k}\left(a_{\Omega_{k}}\right)+\int_{J_{\Omega_{k}}} p_{k} v_{k} w_{k} \mathrm{~d} x\right)
$$

e, assim,

$$
\begin{aligned}
\sum_{k=1}^{r} \int_{\sum_{i i_{k}}}^{r} u^{\prime} w^{\prime} \mathrm{d} x \mathrm{~d} y= & \sum_{k=1}^{r}\left(\left(p_{k} u_{k}^{\prime}\right)\left(b_{\Omega \Omega_{k}}\right) w_{k}\left(b_{\Omega \Omega_{k}}\right)-\left(p_{k} u_{k}^{\prime}\right)\left(a_{\Omega \Omega_{k}}\right) w_{k}\left(a_{\Omega_{k}}\right)\right) \\
& +\sum_{k=1}^{r} \int_{\Omega_{\Omega_{k}}}^{r} v w \mathrm{~d} x \mathrm{~d} y .
\end{aligned}
$$

Ou ainda,

$$
\int_{J_{\Omega}} \nabla u \cdot \nabla w \mathrm{~d} x \mathrm{~d} y=\sum_{k=1}^{r}\left(\left(p_{k} u_{k}^{\prime}\right)\left(b_{\Omega_{k}}\right) w_{k}\left(b_{\Omega_{k}}\right)-\left(p_{k} u_{k}^{\prime}\right)\left(a_{\Omega_{k}}\right) w_{k}\left(a_{\Omega_{k}}\right)\right)+\int_{j_{\Omega}} v w \mathrm{~d} x \mathrm{~d} y .
$$

Como $A_{0} u=v$, temos que

$$
\sum_{k=1}^{r}\left(\left(p_{k} \cdot u_{k}^{\prime}\right)\left(b_{\Omega_{k}}\right) w_{k}\left(b_{\Omega_{k}}\right)-\left(p_{k} u_{k}^{\prime}\right)\left(a_{\Omega_{k}}\right) w_{k}\left(a_{\Omega_{k}}\right)\right)=0 .
$$

Pela prova da Proposição 6.2.7, vemos que, para cada $k=1, \ldots, r, w_{k}\left(a_{\Omega_{k}}\right)=$ $\tilde{w}\left(a_{\Omega_{k}}, y_{k}^{-}\right)$e $w_{k}\left(b_{\Omega_{k}}\right)=\tilde{w}\left(b_{\Omega_{k}}, y_{k}^{+}\right)$, onde $\hat{w}$ é a cxtensão contínua do $w$ a $\Omega$ c $y_{k}^{-}$e $y_{k}^{+}$são números arbitrários tais que $\left(a_{\Omega_{k}}, y_{k}^{-}\right) \in \bar{\Omega}_{k} \cap\left(\left\{a_{\Omega_{k}}\right\} \times \mathbb{R}\right) \mathrm{e}\left(b_{\Omega_{k}}, y_{k}^{-}\right) \in \bar{\Omega}_{k} \cap\left(\left\{b_{\Omega_{k}}\right\} \times \mathbb{R}\right)$.

Seja $Q$ uma componente conexa de $E$ e escolhamos $w$ tal que $\left.\tilde{w}\right|_{Q}=1$ e $\left.\tilde{w}\right|_{E \backslash Q}=0$. Tal cscolha é possivel pela Proposição 6.2.7. Para essa particular escolha de $w$ obtemos

$$
\begin{aligned}
\sum_{k=1}^{r}\left(\left(p_{k} u_{k}^{\prime}\right)\left(b_{\Omega_{k}}\right) w_{k}\left(b_{\Omega_{k}}\right)\right. & \left.-\left(p_{k} u_{k}^{\prime}\right)\left(a_{\Omega_{k}}\right) w_{k}\left(a_{\Omega_{k}}\right)\right) \\
& =\sum_{k=1}^{r}\left(\left(p_{k} u_{k}^{\prime}\right)\left(b_{\Omega_{k}}\right) \tilde{w}\left(b_{\Omega_{k}}, y_{y_{c}}\right)-\left(p_{k} \cdot u_{k}^{\prime}\right)\left(a_{\Omega_{k}}\right) \tilde{w}\left(a_{\Omega_{k}}, y_{k}^{-}\right)\right) \\
& =\sum_{k \in \sigma_{+}(Q)}\left(p_{k} u_{k}^{\prime}\right)(c)-\sum_{k \in \sigma_{-}(Q)}\left(p_{k} u_{k}^{\prime}\right)(c) .
\end{aligned}
$$

Segue da igualdade (6.7) que

$$
\sum_{k \in \sigma+(Q)}\left(p_{k} u_{k}^{\prime}\right)(c)=\sum_{k \in \sigma \ldots(Q)}\left(p_{k} u_{k}^{\prime}\right)(c)
$$


provando (c)

Suponhamos que as hipóteses de (2) estejam satisfeitas.

Definimos $u(x, y)=u_{k}(x), w(x, y)=u_{k}^{\prime}(x)$ e $v(x, y)=v_{k}(x)$ para $k=1, \ldots, r$, e $(x, y) \in \Omega_{l}$. Como $Z$ tem medida nula em $\mathbb{R}^{2}$, temos que $u, v$ e $w$ estão ben-definidas quase sempre e pela parte (a)

$$
\int_{J_{\Omega}}^{r} u^{2} \mathrm{~d} x \mathrm{~d} y=\sum_{k=1}^{r} \int_{j J_{\Omega_{k}}}^{n} p_{k} u_{k}^{2} \mathrm{~d} x
$$

isto é, $u \in L^{2}(\Omega)$. De modo análogo mostramos que $v, w \in L^{2}(\Omega)$

Vamos mostrar que $u \in H_{s}^{1}(\Omega)$ com $\partial_{x} u=w$. Para isso, mostremos que, para todo $\left(x_{0}, y_{0}\right) \in \Omega$, , existe um $\xi>0$ tal que $I_{\xi}\left(x_{0}\right) \times I_{\xi}\left(y_{0}\right) \subset \Omega$ e que, para quaisquer que sejam $\phi \in \mathcal{D}\left(I_{\xi}\left(x_{0}\right)\right)$ e $\psi \in \mathcal{D}\left(I_{\xi}\left(y_{0}\right)\right)$, temos que

$$
\int_{\Omega} u \phi^{\prime} \psi \mathrm{d} x \mathrm{~d} y=-\int_{J \Omega} w \phi \psi \mathrm{d} x \mathrm{~d} y
$$

$$
\int_{\Omega} u \phi \psi^{\prime} \mathrm{d} x \mathrm{~d} y=0
$$

Consideremos dois casos:

Caso 1: Suponhamos que $\left(x_{0}, y_{0}\right) \in \bigcup_{k-1}^{r} \Omega_{k}$. Nesse caso $\partial_{x} u\left(x_{0}, y_{0}\right)=w\left(x_{0}, y_{0}\right) \mathrm{e}$ $\partial_{y} u\left(x_{0}, y_{0}\right)=0$. Portanto, as igualdades $(6.8)$ e $(6.9)$ estão verificadas.

Caso 2: Suponhamos que $\left(x_{0}, y_{0}\right) \in \Omega \backslash\left(\bigcup_{k=1}^{r} \Omega_{k}\right)$ seja arbitrário. Segue que $\left(x_{0}, y_{0}\right) \in$ $Z$. Portanto, existe um $\xi>0$ tal que $I_{\xi}\left(x_{0}\right) \times I_{\xi}\left(y_{0}\right) \subset \Omega$ e $\left(I_{\xi}\left(x_{0}\right) \times I_{\xi}\left(y_{0}\right)\right) \cap Z \subset\left\{x_{0}\right\} \times \mathbb{R}$. Com isso, $I_{\xi}^{-}\left(x_{0}\right) \times I_{\xi}\left(y_{0}\right) \subset \bigcup_{k=1}^{r} \Omega_{k}$ e $I_{\xi}^{+}\left(x_{0}\right) \times I_{\xi}\left(y_{0}\right) \subset \bigcup_{k=1}^{r} \Omega_{k}$. Como $I_{\xi}^{-}\left(x_{0}\right) \times I_{\xi}\left(y_{0}\right)$ é conexo, existe um $k \in\{1, \ldots, r\}$ tal que $I_{\xi}^{-}\left(x_{0}\right) \times I_{\xi}\left(y_{0}\right) \subset \Omega_{k}$. Similarmente, existe um $l \in\{1, \ldots, r\}$ tal que $I_{\xi}^{+}\left(x_{0}\right) \times I_{\xi}\left(y_{0}\right) \subset \Omega_{l}$.

Afirmamos que $k \neq l$. De fato, caso contrário, $\left(x_{0}, y_{0}\right) \in \dot{\partial} \Omega_{k} \cap\left\{a_{\Omega_{k}}, \dot{o}_{\Omega_{k}}\right\} \times \mathbb{R}$ e então $x_{0} \in\left\{a_{\Omega_{k}}, b_{\Omega_{k}}\right\}$. Entretanto, se $x_{0}=a_{\Omega_{k}}$, temos que $I_{\xi}^{-}\left(x_{0}\right) \times I_{\xi}\left(y_{0}\right)$ é disjunto de $\jmath_{\iota_{k}}$ e se $x_{0}=b_{\Omega_{k}}$, segue que $i_{\dot{\xi}}^{+\frac{1}{c}}\left(x_{0}\right) \times I_{\xi}\left(y_{0}\right)$ é disjunto de $\Omega_{k}$. Em ambos os casos temos uma contradição, já que $I_{\xi}^{-}\left(x_{0}\right) \times I_{\xi}\left(y_{0}\right) \subset \Omega_{k} \in I_{\xi}^{+}\left(x_{0}\right) \times I_{\xi}\left(y_{0}\right) \subset \Omega_{l}$.

Assim, $\Omega_{k}$ se une a $\widehat{\jmath}$ ! no ponto $x_{0}$ em $\Omega$ e a parte (b) de (2) implica que $u_{k}\left(x_{0}\right)=$ $u_{l}\left(x_{0}\right)$.

Sejam $\phi \in \mathcal{D}\left(I_{\xi}\left(x_{0}\right)\right)$ e $\psi \in \mathcal{D}\left(I_{\xi}\left(y_{0}\right)\right)$ funções arbitrárias. Temos

$$
\begin{aligned}
\int_{I_{\Omega}} u \phi^{\prime} \psi \mathrm{d} x \mathrm{~d} y & =\int_{I_{\xi}^{-}\left(x_{0}\right) \times I_{\xi}\left(y_{0}\right)} u \phi^{\prime} \psi \mathrm{d} x \mathrm{~d} y+\int_{I_{\xi}^{+}\left(x_{0}\right) \times I_{\xi}\left(y_{0}\right)} u \phi^{\prime} \psi \mathrm{d} x \mathrm{~d} y \\
& =\int_{I_{\xi}\left(y_{0}\right)} \psi\left(\int_{I_{\xi}^{-}\left(x_{0}\right)} u \phi^{\prime} \mathrm{d} x\right) \mathrm{d} y+\int_{I_{\xi}\left(y_{0}\right)} \psi\left(\int_{I_{\xi}^{+}\left(x_{0}\right)} u \phi^{\prime} \mathrm{d} x\right) \mathrm{d} y \\
& =\left(i_{I_{I_{\xi}^{-}\left(x_{0}\right)}} u_{k} \phi^{\prime} \mathrm{d} x+\int_{J_{I_{\xi}^{+}\left(x_{0}\right)}} u_{l} \phi^{\prime} \mathrm{d} x\right) \int_{I_{\xi}\left(y_{0}\right)} \psi \mathrm{d} y .
\end{aligned}
$$


Integrando por partes a última expressão acima e usando o fato de $\phi \in \mathcal{D}\left(I_{\xi}\left(x_{0}\right)\right)$, obtemos

$$
\begin{aligned}
\int_{\Omega} u \phi^{\prime} \psi \mathrm{d} x \mathrm{~d} y & =-\left(\int_{I_{\xi}^{-}\left(x_{0}\right)} u_{k}^{\prime} \phi \mathrm{d} x+\int_{I_{\xi}^{+}\left(x_{0}\right)} u_{l}^{\prime} \phi \mathrm{d} x\right) \int_{I_{\xi}\left(y_{0}\right)} \psi \mathrm{d} y \\
& =\left(-\int_{I_{\xi}\left(x_{0}\right)} w \phi \mathrm{d} x\right) \int_{I_{\xi}\left(y_{0}\right)} \psi \mathrm{d} y=-\int_{\Omega} w \phi \psi \mathrm{d} x \mathrm{~d} y .
\end{aligned}
$$

Isso mostra a igualdade (6.8). Como $\psi \in \mathcal{D}\left(I_{\xi}\left(y_{0}\right)\right)$, temos que $\int_{I_{\xi}\left(y_{0}\right)} \psi^{\prime} \mathrm{d} y=0$. Portanto,

$$
\int_{\Omega 2} u \phi \psi^{\prime} \mathrm{d} x \mathrm{~d} y=\int_{I_{\xi}^{-}\left(x_{0}\right) \times I_{\xi}\left(y_{0}\right)}^{f} u \phi \psi^{\prime} \mathrm{d} x \mathrm{~d} y+\int_{I_{\xi}^{+}\left(x_{0}\right) \times I_{\xi}\left(y_{0}\right)} u \phi \psi^{\prime} \mathrm{d} x \mathrm{~d} y=0 .
$$

O que conclui a demonstração de (6.9). Logo, $u \in H_{s}^{1}(\Omega)$.

Afirmamos que $v \in L_{s}^{2}(\Omega)$. Para cada $k=1, \ldots, r$, o Lema 6.1.3 implica que existe uma sequência $\left(\phi_{n}^{k}\right)_{n \in \mathbb{N}} \mathrm{cm} \mathcal{D}\left(J_{\Omega_{k}}\right)$ tal que

$$
\lim _{n \rightarrow \infty} \int_{J_{\Omega_{k}}} p_{k}\left(v_{k}-w_{n}^{\prime}\right) ; n=0
$$

Dado $n \in \mathbb{N}$, definimos a função $\xi_{n}: \Omega \longrightarrow \mathbb{R}$ por $\xi_{n}(x, y):=\phi_{n}^{k}(x),(x, y) \in \Omega_{l_{1}}$ e $k=1, \ldots, r$. O mesmo raciocínio que utilizamos para demonstrar que $u \in I_{s}^{1}(\Omega)$ permite concluir que $\xi_{n} \in H_{s}^{i}(s i)$ para todo $n \in \mathbb{N}$. Além disso,

$$
\int_{\Omega}\left|v-\xi_{n}\right|^{2} \mathrm{~d} x \mathrm{~d} y=\sum_{k=1}^{r} \int_{\Omega_{\Omega_{k}}}\left|w-\xi_{n}\right|^{2} \mathrm{~d} x \mathrm{~d} y=\sum_{k=1}^{r} \int_{J_{\Omega_{k}}} p_{k}\left(v_{k}-\phi_{n}^{k}\right)^{2} \mathrm{~d} x .
$$

Portanto, $\xi_{n} \rightarrow v$ em $L^{2}(\Omega)$. Assim, $v \in L_{s}^{2}(\Omega)$.

Para concluirmos a demonstração do teorema devemos mostrar que $u \in D\left(A_{0}\right)$ e que $A_{0} u=v$.

Seja $w \in H_{s}^{1}(\Omega) \cap C^{0}(\Omega)$ arbitrário. Consideremos $w_{k}, k=1, \ldots, r$, e $\tilde{w}$ como na Proposição 6.2.7. Com a mesma notação da primeira parte da demonstração deste teorema, temos que

$$
\begin{aligned}
\sum_{k=1}^{r}\left(\left(p_{k} u_{k}^{\prime}\right)\right. & \left.\left(b_{\Omega_{k}}\right) w_{k}\left(b_{\Omega_{k}}\right)-\left(p_{k} u_{k}^{\prime}\right)\left(a_{\Omega_{k}}\right) w_{k}\left(a_{\Omega_{k}}\right)\right) \\
& =\sum_{k=1}^{r}\left(\left(p_{k} u_{k}^{\prime}\right)\left(b_{\Omega_{k}}\right) \tilde{w}\left(b_{\Omega_{k}}, y_{k}^{+}\right)-\left(p_{k} u_{k}^{\prime}\right)\left(a_{\Omega_{k}}\right) \tilde{w}\left(a_{\Omega_{k}}, y_{k}\right)\right) \\
& =\sum_{Q \in \mathcal{P}}\left(\sum_{k \in \sigma+(Q)}\left(p_{k} \cdot u_{k}^{\prime}\right)\left(c_{Q}\right) \tilde{w}\left(c_{Q}, y_{k}^{+}\right)-\sum_{k \in \sigma_{-}(Q)}\left(p_{k} u_{k}^{\prime}\right)\left(c_{Q}\right) \tilde{w}\left(c_{Q}, y_{k}^{-}\right)\right),
\end{aligned}
$$

onde $\mathcal{P}$ é o conjunto finito de componentes conexas de $E$ e, para cada $Q \in \mathcal{P}, c_{Q}$ é o único número tal que $Q \subset\left\{c_{Q}\right\} \times \mathbb{R}$. Como $\tilde{w}$ ć constante crn cada $Q \in \mathcal{P}$, segue de (c) 
e de (6.10) que

$$
\sum_{k=1}^{r}\left(\left(p_{k} u_{k}^{\prime}\right)\left(b_{\Omega_{k}}\right) w_{k}\left(b_{\Omega_{k}}\right)-\left(p_{k} u_{k}^{\prime}\right)\left(a_{\Omega_{k}}\right) w_{k}\left(a_{\Omega_{k}}\right)\right)=0 .
$$

Assim,

$$
\begin{aligned}
\int_{\Omega} \nabla u \cdot \nabla w \mathrm{~d} x \mathrm{~d} y & =\sum_{k=1}^{r} \int_{\Omega_{k}} \nabla u_{k} \cdot \nabla w_{k} \mathrm{~d} x \mathrm{~d} y=\sum_{k=1}^{r} \int_{J_{\Omega_{k}}} p_{k} u_{k}^{\prime} w_{k}^{\prime} \mathrm{d} x \\
& =\sum_{k=1}^{r}\left(\left(p_{k} u_{k}^{\prime}\right)\left(b_{\Omega_{k}}\right) w_{k}\left(b_{\Omega_{k}}\right)-\left(p_{k} u_{k}^{\prime}\right)\left(a_{\Omega_{k}}\right) w_{k}\left(a_{\Omega_{k}}\right)-\int_{J_{\Omega_{k}}}\left(p_{k} u_{k}^{\prime}\right)^{\prime} w_{k} \mathrm{~d} x\right) \\
& =-\sum_{k=1}^{r} \int_{J_{1 s_{k}}}\left(p_{k} u_{k}^{\prime}\right)^{\prime} w_{k} \mathrm{~d} x=\sum_{k=1}^{r} \int_{J_{\Omega_{k}}} p_{k} v_{k} w_{k} \mathrm{~d} x=\int_{\Omega} v w \mathrm{~d} x \mathrm{~d} y .
\end{aligned}
$$

Como $H_{s}^{1}(\Omega) \cap C^{0}(\Omega)$ é um conjunto denso em $H_{s}^{1}(\Omega)$, segue que

$$
\int_{\Omega}^{R} \nabla u \cdot \nabla v \mathrm{~d} x \mathrm{~d} y=\int_{\Omega} v w \mathrm{~d} x \mathrm{~d} y \text { para todo } w \in H_{s}^{1}(\Omega) .
$$

Logo, (1) está demonstrado.

Seja $\Omega$ um domínio bem decomposto e $\Omega_{1}, \ldots, \Omega_{r}$ uma boa decomposição deste domínio. Suponhamos que $f: \mathbb{R} \longrightarrow \mathbb{R}$ seja uma função satisfazendo as hipóteses (HC1). Com isso, temos que a equação abstrata

$$
\dot{u}+A_{0} u=\hat{f}(u)
$$

está bem definida. Então, o Teorema 6.2 .8 sugere que esta equação é equivalente ao seguinte sistema unidimensional de equações do reação-difusão:

$$
u_{k}^{\prime}=\left(1 / p_{k}\right)\left(p_{k} u_{k}^{\prime}\right)^{\prime}+\int\left(u_{k}\right) \text { em } J_{\Omega_{k}}, \text { para } k=1, \ldots, r,
$$

com as condições de compatibiliclade

$$
u_{k}(c)=u_{l}(c)
$$

sempre que $b_{\Omega_{k}}=a_{s_{l}}=c$ e $\Omega_{k}$ e $\Omega_{l}$ se unem no ponto $c$. Além disso, sempre que $Q$ é uma componente conexa de $E$, temos as seguintes condições, conhecidas como condições de balanço do tipo Kirchhoff:

$$
\sum_{k \in \sigma_{+}(Q)}\left(p_{k} u_{k}^{\prime}\right)(c)=\sum_{k \in \sigma_{-}(Q)}\left(p_{k} \cdot u_{k}^{\prime}\right)(c)
$$

Observemos que este sistema pode ser interpretado como uma equação de reação-difusão em um grafo. As arestas desse grafo são os intervalos ${ }^{-} J_{\Omega_{k}}$, para $k=1, \ldots, r$ e os vértices são os extremos destes intervalos. Além disso, cada intervalo deverá ser repetido no grafo o número de vezes que ele aparece na sequência $\left(J_{\Omega_{k}}\right)_{k=1}^{r}$. Os vértices por sua vez serão repetidos o número de vezes que ele ocorre como projeção de uma componente conexa de E. A Figura 4 apresenta o que seria o grafo no caso do domínio bem decomposto da Figura 3. 


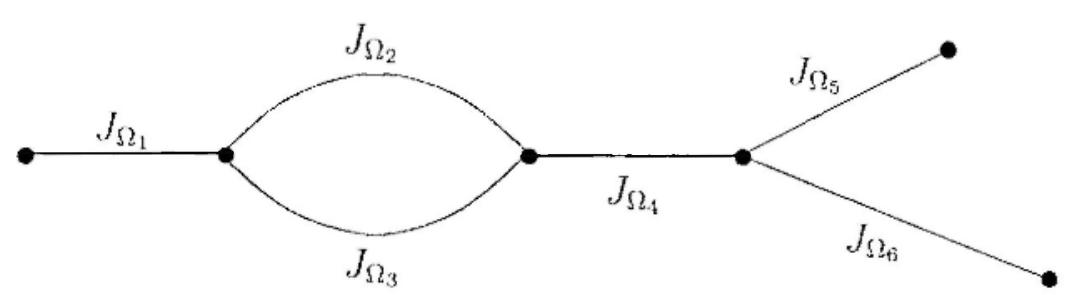

Figura 4: o grafo associado ao domínio $\Omega$ da Figura 3.

Exemplo 6.2.9 (Conjuntos Ordenados) Vamos analisar um caso particular dos domínios considerados em [14]. Seja $J=(a, b)$ com $-\infty<a<b<\infty$. Consideremos uma função $g:[a, b] \longrightarrow \mathbb{R}$ tal que $g \in C^{1}(a, b) \cap C^{0}([a, b])$ e $g(x)>0$ para todo $x \in[a, b]$. Definimos:

$$
\Omega:=\left\{(x, y) \in \mathbb{R}^{2} \mid x \in J \text { e } 0<y<g(x)\right\}
$$

e para cada $\varepsilon>0$ definimos:

$$
\Omega_{\varepsilon}:=\left\{(x, y) \in \mathbb{R}^{2} \mid x \in J \text { e } 0<y<\varepsilon g(x)\right\} .
$$

Observemos que, quando $\varepsilon \rightarrow 0$, temos que $\Omega_{r}$ colapsa no intervalo $J$.

Consideremos a equação de reação-difusão em $\Omega_{\varepsilon}$

$$
\begin{array}{ll}
u_{t}=\Delta u+f(u), & t>0,(x, y) \in \Omega_{\varepsilon}, \\
\partial_{\nu_{\varepsilon}} u=0, & \iota>0,(x, y) \in \partial \Omega_{\varepsilon} .
\end{array}
$$

Neste caso, para cada $x \in[a, b], g(x)$ c a medida da seção vertical $U_{x}=\{y \in \mathbb{R} \mid$ $(x, y) \in \Omega\}$. Com isso, aplicando o Teorema 6.2.8, temos que as soluções de $\left(E_{\varepsilon}\right)$ devem ser comparadas com as solıções da seguinte equação $\mathrm{cm} J$ :

$$
u_{t}=\frac{1}{g}\left(g u^{\prime}\right)^{\prime}+\hat{f}(u)
$$

e com as condições de fronteira

$$
u^{\prime}(a)=u^{\prime}(b)=0
$$

que é justamente o que foi apresentado em [14]. Observamos que, pela teorì de SturmLiouville, os autovalores da equção limite acima têm multiplicidade simples.

O Teorema 6.2.8 nos possibilita analisar com mais detalhes os espaços $H_{s}^{1}(\Omega)$ e $L_{s}^{2}(\Omega)$.

No restante desta seção consideraremos um domínio bem decomposto $\Omega$ e $\Omega_{1}, \ldots, \Omega_{r}$ uma boa decomposição deste domínio. Começamos observando que:

Lema 6.2.10 Para cada $k=1, \ldots, r$, as seguintes propriedades são satisfeitas:

(1) se $u \in L_{s}^{2}(\Omega)$, então $\left.u\right|_{\Omega_{k}} \in L_{s}^{2}\left(\Omega_{k}\right)$; 
(2) se $u \in H_{s}^{1}(\Omega)$, entâo $\left.u\right|_{\Omega_{u}} \in H_{s}^{1}\left(\Omega_{k}\right)$.

Demonstração. É claro que se $u \in H_{s}^{1}(\Omega)$, então $\left.u\right|_{\Omega_{*}} \in H_{s}^{1}\left(\Omega_{k}\right)$. Suponhamos então que $u \in L_{s}^{2}(\Omega)$. Então existe uma sequência $\left(u_{n}\right)_{n \in \mathbb{N}}$ em $H_{s}^{1}\left(\Omega_{k}\right)$ tal que $u_{n}$ converge para $u$ na norma de $L^{2}(\Omega)$ quando $n \rightarrow \infty$. Neste caso, para cada $k-1, \ldots, r,\left.u_{*}\right|_{\Omega_{r}}$ converge para $\left.u\right|_{\hat{\Omega}_{\tau_{k}}}$ na norma de $L^{2}\left(\Omega_{k}\right)$ quando $n \rightarrow \infty$, mostrando que $\left.u\right|_{\Omega_{\Omega_{k}}} \in L_{s}^{2}\left(\Omega_{k}\right)$.

Sendo $\Omega_{1}, \ldots, \Omega_{r}$ uma boa decomposição de $\Omega$, temos que $1 / p_{k} \in L^{1}\left(J_{\Omega_{k}}\right)$ para $k=$ $1, \ldots, r$. Assim, o Lema 6.1.2 e a Proposição 6.1 .1 implicam que os espaços $H_{p_{k}}$ e $V_{p_{k}}$ associados às funções $p_{k}$ estão bem definidos para cada $k=1, \ldots, r$. Já vimos que estes espaços são espaços de Hilbert com produtos internos dados respectivamente por:

$$
\langle u, v\rangle_{H_{p_{k}}}:=\int_{J_{S_{k}}} p_{k}(x) u(x) v(x) \mathrm{d} x, \text { para } u, v \in H_{p_{k}}
$$

$$
\langle u, v\rangle_{V_{p_{k}}}:=\int_{J_{\Omega_{k}}} p_{k}(x) u^{\prime}(x) v^{\prime}(x) \mathrm{d} x+\int_{J_{\Omega_{k}}} p_{k}(x) u(x) v(x) \mathrm{d} x, \text { para } u, v \in V_{p_{k}} .
$$

Além disso, a inclusão $V_{p_{k}} \hookrightarrow H_{p_{k}}$ é densa e compacta para cadla $k=1, \ldots, r$.

Definimos agora os espaços

$$
I_{\oplus}:=I_{p_{1}} \oplus \ldots \oplus I_{p_{r}}:-\left\{[u]=\left(u_{1}, \ldots, u_{r}\right) \mid u_{k} \in I I_{p_{k}}, k:=1, \ldots, r\right\}
$$

$$
V_{\oplus}:=V_{p_{1}} \oplus \ldots \ominus V_{p_{r}}:=\left\{[u]=\left(u_{1}, \ldots, u_{r}\right) \mid u_{k} \in V_{p_{k}}, k=1, \ldots, r\right\} .
$$

O Lema 6.2.10 implica que estes espaços estão bem-definidos.

Definimos em $H_{\ominus}$ e $V_{\oplus}$ os produtos

$$
\langle[u],[v]\rangle_{H_{\oplus}}:=\sum_{k=1}^{r}\left\langle\left\langle u_{k}, v_{k}\right\rangle_{H_{p_{k}}}, \text { para } u, v \in I_{\oplus},\right.
$$

$\mathrm{e}$

$$
\langle[u],[v]\rangle_{V_{\oplus}}:=\sum_{k=1}^{r}\left\langle u_{k}, v_{k}\right\rangle_{V_{\mathcal{P}_{k}}}, \text { para } u, v \in V_{\oplus} .
$$

Temos o seguinte resultado:

Lema 6.2.11 Os produtos $\langle\cdot, \cdot\rangle_{H_{\oplus}} e\langle\cdot, \cdot\rangle_{V_{\ominus}}$ definem uma estrutura de espaço de Hilbert em $I_{\ominus}$ e $V_{\oplus}$ respectivamente. Além disso, a inclusũo $V_{\oplus} \leftrightarrow I I_{\oplus}$ é densa e compacta.

Demonstração. É claro que $\langle\cdot, \cdot\rangle_{I_{\oplus}}$ e $\langle\cdot, \cdot\rangle_{V_{\oplus}}$ definem uma estrutura de espaço de Hilbert em $H_{\oplus}$ e $V_{\oplus}$ respectivamente. Para provarmos que $V_{\oplus} \hookrightarrow H_{\oplus}$ com imersão densa e compacta, basta lembrarmos que, pela Proposição 6.1.8, para cada $k=1, \ldots, r$, a imersão $V_{p_{k}} \hookrightarrow H_{p_{k}}$ é densa e compacta. 
Lembremos que, para cada $k=1, \ldots, r$, a aplicação $\imath_{k}: L_{s}^{2}\left(\Omega_{k}\right) \longrightarrow H_{p_{k}}$ dada por $\imath_{k}(u)=v$, onde $v$ é a função dada pela Proposição 6.1.1, é uma isometria de $L_{s}^{2}\left(\Omega_{k}\right)$ sobre $H_{p_{k}}$. Além disso, $\imath_{k}$ restrita a $H_{s}^{1}\left(\Omega_{k}\right)$ é uma isometria sobre $V_{p_{k}}$.

Definimos a aplicação $\imath_{\oplus}: L_{s}^{2}(\Omega) \longrightarrow H_{\oplus}$ por $\imath_{\oplus}(u)=\left(\imath_{1}\left(\left.u\right|_{\Omega_{r}}\right), \ldots, v_{r}\left(\left.u\right|_{\Omega_{r}}\right)\right)$. Segue do Lema 6.2.10 que $\imath_{\oplus}$ está bom definida.

Lema 6.2.12 A aplicação $\imath_{\oplus}: I_{s}^{2}(\Omega) \longrightarrow H_{\oplus}$ é uma isometria de $L_{s}^{2}(\Omega)$ em $H_{\oplus}$. Além disso, $\imath_{\oplus}$, restrita a $H_{s}^{1}(\Omega)$ i uma isometria em $V_{\oplus}$.

Demonstração. Para mostrar que $\imath_{\emptyset}: L_{s}^{2}(\Omega) \longrightarrow H_{\emptyset}$ é uma isometria de $L_{s}^{2}(\Omega)$ em $H_{\emptyset}$, basta usar que $\imath_{k}: L_{s}^{2}\left(\Omega_{k}\right) \longrightarrow H_{p_{k}}$ é uma isometria e proceder como na demonstração do Lema 6.1.6. Analogamente demonstramos que $\imath_{\oplus}$ restrita a $H_{s}^{1}(\Omega)$ é uma isometria em $I I_{\text {今. }}$

Definimos agora

$\tilde{V}_{\oplus}:=\left\{[u] \in V_{\oplus} \mid u_{k}\left(b_{\Omega_{k}}\right)=u_{l}\left(a_{\Omega_{l}}\right)\right.$ sempre que $b_{\Omega_{k}}=a_{\Omega_{l}}=c$ e $\Omega_{k}$ e $\Omega_{l}$ se unem em $\left.c\right\}$.

O scguinte resultado está implicitamente contido na demonstração do Tcorema 6.2.8. Ele apresenta uma caracterização dos espaços $H_{s}^{1}(\Omega)$ e $L_{s}^{2}(\Omega)$.

Proposição 6.2.13 As seguintes propriedades são válidas:

(1) $\imath_{\oplus}\left(L_{s}^{2}(\Omega)\right)=H_{\oplus}$;

(2) $\imath_{\oplus \rightarrow}\left(I I_{s}^{1}(\Omega)\right)=\tilde{V}_{\oplus \rightarrow}$.

Demonstração. Mostremos (2). Seja $u \in H_{s}^{1}(\Omega)$. Então $\imath_{\oplus}(u) \in V_{\oplus}$. Suponhamos que cxistan $k, l \in\{1, \ldots, r\}$ tais que $b_{\Omega_{k}}=a_{\Omega_{l}}=c$ e $\Omega_{k}$ e $\Omega_{l}$ se unem no ponto $c$. Precisamos mostrar que $\imath_{k}\left(\left.u\right|_{\Omega_{k}}\right)(c)=u_{l}\left(\left.u\right|_{\Omega_{l}}\right)(c)$. Para isso definimos $v_{k}:=u_{k}\left(\left.u\right|_{\Omega_{2}}\right.$ ) e $v_{l}:=u_{l}\left(\left.u\right|_{\Omega_{l}}\right)$. Então, pela demonstração da Proposição 6.2 .7 temos que $v_{k}(c)=v_{l}(c)$. Logo, $\imath_{\oplus}(u) \in \tilde{V}_{\oplus}$.

Por outro lado, seja $[u] \in \tilde{V}_{\oplus}$. Mostremos que existe um $v \in H_{s}^{1}(\Omega)$ tal que $\imath_{\oplus}(v)=[u]$. Definimos $v: \Omega \longrightarrow \mathbb{R}$ por

$$
v(x, y):= \begin{cases}u_{k}(x), & \text { para }(x, y) \in \Omega_{k}, k=1, \ldots, r, \\ 0, & \text { para }(x, y) \in \Omega \backslash\left(\bigcup_{k=1}^{r} \Omega_{k}\right) .\end{cases}
$$

Como na prova do Teorema 6.2.8, temos que $v \in H_{o}^{1}(\Omega)$. Alćm disso, $v_{x}(x, y)=u_{k}^{\prime}(x)$ para quase tódo $(x, y) \in \Omega_{k} \operatorname{com} k=1, \ldots, r$ e $v_{y}(x, y)=0$ para quase todo $(x, y) \in \Omega$. Pela construģão de $v$ temos que $t_{\circledast}(v)=[u]$. Isso conclui a demonstração de (2).

Vamos mostrar $(1)$. Temos que $\imath_{\oplus}\left(L_{s}^{2}(\Omega)\right) \subset H_{\oplus}$. Seja $[u] \in H_{\oplus}$. Precisamos mostrar que existe um $v \in L_{s}^{2}(\Omega)$ tal que $\imath_{\oplus}(v)=[u]$. Definimos $v: \Omega \longrightarrow \mathbb{R}$ por:

$$
,(x, y):= \begin{cases}u_{k}(x), & \text { para }(x, y) \in \Omega_{k}, k=1, \ldots, r, \\ 0, & \text { para }(x, y) \in \Omega \backslash\left(\bigcup_{k=1}^{r} \Omega_{k}\right) .\end{cases}
$$


Como $u_{k} \in H_{p_{k}}$ para cada $k=1, \ldots$ segue que $v \in L^{2}(\Omega)$. Afirmamos que $v \in L_{s}^{2}(\Omega)$. De fato, para cada $k=1, \ldots, r$, pelo Lema 6.1 .3 , existe uma sequência $\left(\phi_{n}^{k}\right)_{n \in \mathbb{N}}$ em $\mathcal{D}\left(J_{\Omega_{k}}\right)$ tal que

$$
\lim _{n \rightarrow \infty} \int_{J_{\Omega_{2}}} p_{k}\left(u_{k}-\phi_{n}^{k}\right)^{2} \mathrm{~d} x=0 .
$$

Como na demonstração do Teorema 6.2 .8 , definimos para cada $n \in \mathbb{N}$ a função $\psi_{n}: \Omega \longrightarrow$ $\mathbb{R}$ por

$$
\psi(x, y):= \begin{cases}\phi_{n}^{k}(x), & \operatorname{para}(x, y) \in \Omega_{k}, k=1, \ldots, r \\ 0, & \operatorname{para}(x, y) \in \Omega \backslash\left(\bigcup_{k=1}^{r} \Omega_{k}\right) .\end{cases}
$$

Segue que $\psi_{n} \in H_{s}^{1}(\Omega)$ para todo $n \in \mathbb{N}$ e a sequência $\left(\psi_{n}\right)_{n \in \mathbb{N}}$ converge para $v$ na norma de $L^{2}(\Omega)$. Portanto, $v \in L_{s}^{2}(\Omega)$. Além disso, do modo que $v$ foi definida temos que $u_{\oplus \rightarrow}(v)=[u]$. Isso conclui a demonstração da proposição.

\subsection{Domínios em forma de $C$}

Nesta seção apresentaremos uma classe bem simples de domínios, os quais satisfazem todas as propricdades que necessitamos para aplicar os resultados da Seção 6.2. Ilustramos como tais resultados permitem uma análise dos autovalores do operador $A_{0}$.

Sejam $a_{i}$ e $b_{i}, i=1,2,3$, números reais com $a_{1}<0, a_{2}>0, a_{3}>0$ e $0<b_{1}<b_{2}<b_{3}$. O domínio em forma de $\left(C^{\prime}\right.$ com parâmetros $a_{i}$ e $b_{i}, i=1,2,3$, é definido como

$$
\Omega:=\left(\left(a_{1}, a_{2}\right) \times\left(0, b_{1}\right)\right) \cup\left(\left(a_{1}, 0\right) \times\left(0, b_{3}\right)\right) \cup\left(\left(a_{1}, a_{3}\right) \times\left(b_{2}, b_{3}\right)\right)
$$

Na Figura 5 temos uma representação geométrica de tal domínio. Definindo $\Omega_{1}:=\left(a_{1}, 0\right) \times$ $\left(0, b_{3}\right), \Omega_{2}:=\left(0, a_{2}\right) \times\left(0, b_{1}\right), \Omega_{3}:=\left(0, a_{3}\right) \times\left(b_{2}, b_{3}\right)$ e $Z:=\{0\} \times \mathbb{R}$, obtemos uma boa decomposição de $\Omega$. Obscrvemos neste caso que $J_{\Omega_{1}}=\left(a_{1}, 0\right), J_{\Omega_{3}}=\left(0, a_{2}\right)$ e $J_{\Omega_{3}}=\left(0, a_{3}\right)$. Além disso, $p_{1}(x)=b_{3}$ para todo $x \in J_{\Omega_{1}}, p_{2}(x)=b_{1}$ para todo $x \in J_{\Omega_{2}}$ e $p_{3}(x)=b_{3}-b_{2}$ para todo $x \in J_{\Omega_{3}}$. O seguinte resultado é uma consequência simples do Teorema 6.2.8.

Proposição 6.3.1 Um $\operatorname{par}(\lambda, u)$ é um par autovalor-autovetor de $A_{0}$ se, e somente se, $u$ é näo-nulo e existirem funçōes $u_{l}: J_{\Omega_{l}} \longrightarrow \mathbb{R}, l=1,2,3$, números reais $\alpha$ e $\beta_{l}, l=1,2,3$, e um conjunto de medida nula $S \subset \mathbb{R}^{2}$ tais que as seguintes propriedades são satisfeitas:

(1) $u(x, y)=u_{l}(x)$ para todo $(x, y) \in \Omega_{l} \backslash S$ e $l \div 1,2,3$;

(2) $u_{l}-\alpha \cos \sqrt{\lambda} x+\beta_{l} \operatorname{sen} \sqrt{\lambda} x$, para todo $x \in J_{l,}$ e $l=1,2,3$;

(3) $b_{3} \sqrt{\lambda} \beta_{1}=b_{1} \sqrt{\lambda} \beta_{2}+\left(b_{3}-b_{2}\right) \sqrt{\lambda} \beta_{3}$;

(4) $-\alpha \sqrt{\lambda} \operatorname{sen} a_{l} \sqrt{\lambda}+\beta_{l} \sqrt{\lambda} \cos a_{l} \sqrt{\lambda}=0$ paral $-1,2,3$. 


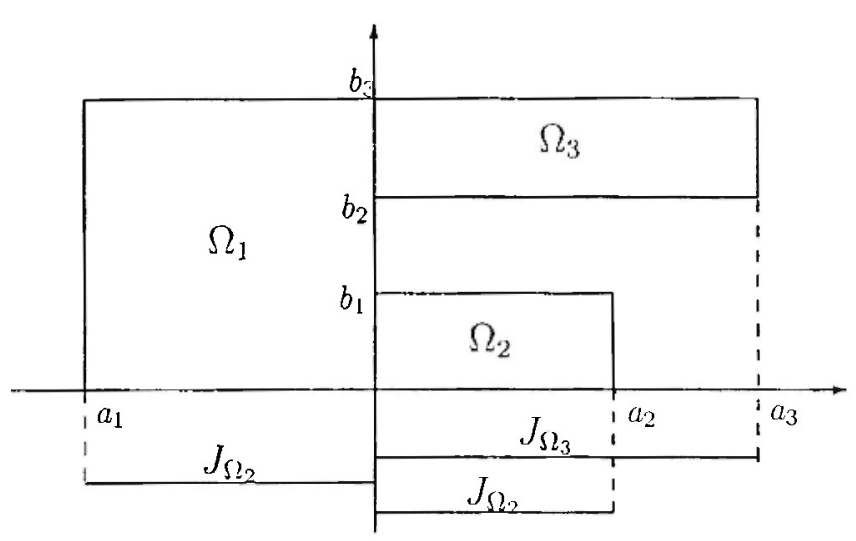

Figura 5: Um domínio em forma de $C$.

Demonstração. O Teorema 6.2.8 implica que $A_{0} u=\lambda u$ se, e somente se, $u$ é não-nulo e existem funçōes absolutamente contínuas $u_{l}: J_{\Omega_{l}} \longrightarrow \mathbb{R}, l=1,2,3$, e um conjunto de medida nula $S \subset \mathbb{R}^{2}$ tal que, sempre que $l=1,2,3$, então $u(x, y)=u_{l}(x)$ para $(x, y) \in \Omega_{l} \backslash S$. Como as funções $p_{l}$ são todas constantes para $l=1,2,3$, temos que $u_{l}, u_{l}^{\prime} \in L^{2}\left(J_{\Omega_{l}}\right)$ para $l=1,2,3$. Também, para $l=1,2,3, u_{l}^{\prime \prime}=\lambda u_{l}$ no sentido das distribuições. Isto nos fornece

$$
u_{l}(x)=\alpha_{l} \cos \sqrt{\lambda} x+\beta_{l} \operatorname{sen} \sqrt{\lambda} x, \quad l=1,2,3 \text { e } x \in J_{\Omega_{l}},
$$

onde $\alpha_{l}$ e $\beta_{l}$ são números reais arbitrários. Como $\widehat{\jmath}_{1}$ e $\Omega_{2}$ se unem no ponto 0 bem como $\Omega_{1}$ o $\Omega_{3: 3}$ o item (b) do Teorema 6.2 .8 implica que

$$
u_{2}(0)=\lim _{x \rightarrow 0} u_{2}(x)=u_{1}(0)=\lim _{x \rightarrow 0} u_{1}(x)=u_{3}(0)=\lim _{x \rightarrow 0} u_{3}(x)
$$

Logo,

$$
\alpha_{1}=\alpha_{2}=\alpha_{3}=: \alpha
$$

ou seja,

$$
u_{l}(x)=\alpha \cos \sqrt{\lambda} x+\beta_{l} \operatorname{sen} \sqrt{\lambda} x, \quad l=1,2,3 \text { e } x \in J_{\Omega_{l}} .
$$

Derivando esta expressão obtemos

$$
u_{l}^{\prime}(x)=-\alpha \sqrt{\lambda} \operatorname{sen} \sqrt{\lambda} x+\beta_{l} \sqrt{\lambda} \cos \sqrt{\lambda} x, \quad l=1,2,3 .
$$

Mas as condições de balanço do item (c) do Teorema 6.2 .8 implicam que

$$
u_{1}^{\prime}\left(a_{1}\right)=u_{2}^{\prime}\left(a_{2}\right)=u_{3}^{\prime}\left(a_{3}\right)=0
$$

e

$$
b_{1} u_{2}^{\prime}(0)+\left(b_{3}-b_{2}\right) u_{3}^{\prime}(0)=b_{3} u_{1}^{\prime}(0) .
$$

A propriedade (3) segue de (6.12) e as igualdades em (6.11) implicam que (4) está verificada. 
Proposição 6.3.2 Seja $\lambda$ um número real.

(1) Se $\lambda=0$, então $\lambda$ é um autovalor simples de $A_{0}$;

(2) se $\lambda>0$ é uma autovalor de $A_{0} \operatorname{com} \operatorname{sen} a_{l} \sqrt{\lambda}=0$ para cada $l=1,2,3$, então $\lambda$ é um autovalor simples de $A_{0}$;

(3) se $\lambda>0$ é uma autovalor de $A_{0}$ com sen $a_{i} \sqrt{\lambda} \neq 0$ para algum $i=1,2,3$, então $\lambda$ possui multiplicidade no máximo 2.

Demonstração. Observemos que a Proposição 6.3 .1 implica que $\lambda=0$ é um autovalor de $A_{0}$, e além disso, para tal autovalor.

$$
u_{l}(x)=\alpha, \quad l=1,2,3,
$$

isto é, $\lambda=0$ é un autovalor simples de $A_{0}$.

Suponhamos agora que $\lambda>0$ é um autovalor arbitrário de $A_{0}$. Se sen $a_{l} \sqrt{\lambda}=0$ para cada $l=1,2,3$, então a propriedade (4) da Proposição 6.3.1 implica que $\beta_{l}=0, l=1,2,3$, e a propriedade (2) nos fornece

$$
u_{l}(x)=\alpha \cos \sqrt{\lambda} x, \quad l=1,2,3 \text { para } x \in J_{\Omega_{l}}
$$

isto é, $\lambda$ é um autovalor simples de $A_{0}$. Se sen $a_{i} \sqrt{\dot{\lambda}} \neq \ddot{u}$ para algum $i=1,2,3$, então $\alpha=$ $\beta_{i} \cot a_{i} \sqrt{\lambda}$ e como, pela propriedade $(3), b_{3} \beta_{1}=b_{1} \beta_{2}+\left(b_{3}-b_{2}\right) \beta_{3}$, segue da propriedade (2) que $\lambda$ possui multiplicidade no máximo 2.

A Proposição 6.3 .2 implica que $A_{0}$ não possui autovalores com multiplicidade maior que 2.

O próximo resultado apresenta um critério de monotonicidade estrita para domínios em forma de $C$. Notemos que não podemos usar o Tcorcma 3.2.2 na Seção 3.2.

Proposição 6.3.3 Sejam $\lambda>0$ um autovalor de $A_{0}$ e $j \in \mathbb{N}, j \geq 2$, tais que $\lambda=\lambda_{0, j}<$ $\lambda_{0, j+1}$. Então as seguintes propriedades são equivalentes:

(1) $\operatorname{sen} a_{l} \sqrt{\lambda}=0$ para $l=1,2,3$;

(2) existe um $\bar{c}_{0}>0$ tal que $\lambda_{\varepsilon, j}=\lambda$ para todo $\varepsilon \in\left[0, \varepsilon_{0}\right]$.

Demonstração. Suponhamos que a afirmativa (1) seja satisfeita. Definimos $u(x, y)=$ $u(x)=\cos \sqrt{\lambda} x$ para $(x, y) \in \Omega$. É claro que $u$ é não-nulo. Fazendo $\alpha=1$ e $\hat{j}_{!}=0$ para $l=1,2,3$, segue da Proposicão 6.3.1 que o $\operatorname{par}(\lambda, u)$ é um par autovalor-autovetor do operador $A_{0}$. Em particular, $u \in H_{s}^{1}(\Omega)$. Assim, utilizando o Teorema 1.4.2 de [23], 
temos que, para toda função $v \in H^{1}(\Omega)$,

$$
\begin{aligned}
\langle\nabla u, \nabla v\rangle & =\sum_{l=1}^{3} \int_{\Omega_{l}} u^{\prime}(x) \dot{\partial}_{x} v(x, y) \mathrm{d} x \mathrm{~d} y \\
& =\sum_{l=1}^{3} \int_{\Omega_{\Omega_{i}}} u^{\prime}(x) v(x, y) \nu_{l}(x, y) \mathrm{d} \sigma-\sum_{l=1}^{3} \int_{j \Omega_{l}} u^{\prime \prime}(x) v(x, y) \mathrm{d} x \mathrm{~d} y \\
& =\sum_{l=1}^{3} \int_{\partial \Omega_{l}} u^{\prime}(x) v(x, y) \nu_{l}(x, y) \mathrm{d} \sigma+\lambda \sum_{!=1}^{3} \int_{\Omega_{l}} u(x) v(x, y) \mathrm{d} x \mathrm{~d} y \\
& =\sum_{l=1}^{3} \int_{\partial \Omega_{l}} u^{\prime}(x) v(x, y) \nu_{l}(x, y) \mathrm{d} \sigma+\lambda\langle u, v\rangle,
\end{aligned}
$$

onde $\sigma$ é a medida de Hausdorf unidimensional e $\nu_{l}(x, y)$ é a componente $x$ da normal exterior a $\partial \Omega_{l}$ em $(x, y) \in \partial \Omega_{l}$

Agora, notemos que $\nu_{l}(x, y)=0$ na componente horizontal de $\partial \Omega_{l}$, enquanto que, por hipótese, $u^{\prime}\left(a_{l}\right)=\operatorname{sen} a_{l} \sqrt{\lambda}=0$ para $(x, y)$ na componente vertical de $\partial \Omega_{l}$. Com isso,

$$
\sum_{l=1}^{3} \int_{\partial \Omega_{l}}^{n} u^{\prime}(x) v(x, y) \nu_{l}(x, y) \mathrm{d} \sigma=0
$$

e portanto,

$$
\langle\nabla u, \nabla v\rangle=\lambda\langle u, v\rangle \text { para qualquer } v \in H^{\mathrm{i}}(\Omega) \text {. }
$$

Uma aplicação do Teorema 3.2.1 implica que a condição (2) está satisfeita.

Assumimos que a propriedade (2) esteja verificada. Segue do Teorema 3.2.1 que existe um $u \in H_{s}^{1}(\Omega)$ não nulo tal que

$$
\langle\nabla u, \nabla v\rangle=\lambda\langle u, v\rangle \text { qualquer que seja } v \in H^{1}(\Omega) .
$$

Em particular, $(\lambda, u)$ é um par autovalor-autovetor de $A_{0}$. Pela Proposição 6.3.1, existem funções $u_{l}: J_{S_{l}} \longrightarrow \mathbb{R}$, números reais $\alpha$ e $\beta_{l}, l=1,2,3$, e um conjunto de medida nula $S \subset \mathbb{R}^{2}$ tais que as condições (1)-(4) daquela proposição são verificadas. Portanto, para qualquer que seja $v \in H^{\prime}(\Omega)$ temos por (6.13) que

$$
\begin{aligned}
& \lambda\langle u, v\rangle=\langle\nabla u, \nabla v\rangle=\sum_{l=1}^{3} \int_{s \Omega_{l}} u_{l}^{\prime}(x) \partial_{x} v(x, y) \mathrm{d} x \mathrm{~d} y \\
& =\sum_{l=1}^{3} \int_{l_{0 ; b_{l}}} u^{\prime}(x) v(x, y) \nu_{l}(x, y) \mathrm{d} \sigma-\sum_{l=1}^{3} \int_{c_{l} l} u_{l}^{\prime \prime}(x) v(x, y) \mathrm{d} x \mathrm{~d} y \\
& =\sum_{i=1}^{3} \int_{i \sigma \Sigma_{l}} u^{\prime}(x) v(x, y) \nu_{l}(x, y) \mathrm{d} \sigma+\lambda \sum_{i=1}^{3} \int_{\Omega_{l}} u(x) v(x, y) \mathrm{d} x \mathrm{~d} y \\
& =\sum_{l=1}^{3} \int_{J j \Omega_{l}} u^{\prime}(x) u(x, y) \nu_{l}(x, y) \mathrm{d} \sigma+\lambda\langle u, v\rangle .
\end{aligned}
$$


Isto implica que

$$
\sum_{l=1}^{3} \int_{v_{\partial \Omega_{l}}} u^{\prime}(x) v(x, y) \nu_{l}(x, y) \mathrm{d} \sigma=0 \text {, qualquer que seja } v \in H^{1}(\Omega) .
$$

Tomemos em (6.14) uma função $v$ que é a restrição a $\Omega$ de uma função teste nãonegativa em $\mathbb{R}^{2}$, cujo suporte está contido na faixa horizontal $\mathbb{R} \times\left(b_{1}, b_{2}\right)$ e que é não-nula em $\{0\} \times\left(b_{1}, b_{2}\right)$. Segue que

$$
\int_{b_{1}}^{\dot{o}_{2}} u_{1}^{\prime}(0) v(0, y) \nu_{1}(0, y) \mathrm{d} \sigma=0
$$

ou seja,

$$
u_{1}^{\prime}(0) \int_{b_{1}}^{i_{2}} v(0, y) \nu_{1}(0, y) \mathrm{d} \sigma=0
$$

Portanto, $u_{1}^{\prime}(0)=0$. Como $u^{\prime}\left(a_{1}\right)=0$ e $\lambda>0$, temos que $\beta_{1}=0$. Similarmente mostramos que $\beta_{2}=\beta_{3}=0$. Com isso $\alpha \neq 0$. A propriedade (4) da Proposição 6.3.1 implica que sen $a_{l} \sqrt{\lambda}=0, l=1,2,3$.

No próximo resultado escolhemos os parâmetros $a_{l}$ e $b_{l}, l=1,2,3$ de modo conveniente e, utilizando as Proposições 6.3.1 e 6.3.2, analisamos a multiplicidade dos autovalores de $A_{0}$.

Proposição 6.3.4 Suponhamos que $b_{l}=l, l=1,2,3$, e que $\lambda$ é um número real arbitrário.

(1) $S e-a_{1}=a_{2}=a_{3}=a$ e se $\cos a \sqrt{\lambda}=0$, enlão $\lambda$ é um autovalor de $A_{0}$ com multiplicidade 2, cujo autoespaço é formado por todas as funções u para as quais existem constantes $\beta_{2}, \beta_{3} \in \mathbb{R}$ tais que

$$
u(x, y):= \begin{cases}(1 / 3)\left(\beta_{2}+\beta_{3}\right) \text { sen } \vee \bar{\lambda} x, & \text { quase sempre em } \Omega_{1}, \\ \beta_{l} \operatorname{sen} \sqrt{\lambda} x, & \text { quase sempre em } \Omega_{l} \text { para } l=2,3 ;\end{cases}
$$

(2) se $-a_{1}=a_{2}=a, \cos a \sqrt{\lambda}=0$ e se $\cos a_{3} \sqrt{\lambda} \neq 0$, então $\lambda$ é um autovalor simples de $A_{0}$ e seu autoespaço é formado por todas as funções u para as quais existe uma constante $\beta_{2} \in \mathbb{R}$ tal que

$$
u(x, y):= \begin{cases}(1 / 3) \beta_{2} \operatorname{sen} \sqrt{\lambda} x, & \text { quase sempre em } \Omega_{1}, \\ \beta_{2} \operatorname{sen} \sqrt{\lambda} x, & \text { quase sempre em } \Omega_{2}, \\ 0, & \text { quase sempre em } \Omega_{3}\end{cases}
$$

(3) se $a_{2}=a_{3}=a, \cos a \sqrt{\lambda} \neq 0$ e se $\cos a_{1} \sqrt{\lambda} \neq 0$, então $\lambda$ e um autovalor simples de $A_{0}$ e seu autoespaço é formado por todas as funções u para as quais existe uma 
constante $\beta_{2} \in \mathbb{R}$ tal que

$$
u(x, y):= \begin{cases}0, & \text { quase sempre em } \Omega_{1}, \\ \beta_{2} \operatorname{sen} \sqrt{\lambda} x, & \text { quase sempre em } \Omega_{2} . \\ -\beta_{2} \operatorname{sen} v^{\prime} \lambda x, & \text { quase sempre em } \Omega_{3} .\end{cases}
$$

Em todos os casos, se $j \in \mathbb{N}$ é tal que $\lambda=\lambda_{0, j}<\lambda_{0,1+1}$, então a família $\left(\lambda_{\varepsilon, 3}\right)_{\varepsilon>0}$ é estritamente monótona decrescenle e, em particular, $\lambda_{\varepsilon, 3}<\lambda$ para todo $\varepsilon>0$.

Demonstração. Mostremos (1). Se u é uma função definidla como em (1), então as condiçóes da Proposição 6.3 .1 estão satisfeitas com $\alpha=0$ e $\beta_{1}=(1 / 3)\left(\beta_{2}+\beta_{3}\right)$. Tal proposição implica que $\lambda$ é um autovalor de $A_{0}$ e seu autoespaço é formado por todas as funçōes $u$ dessa forma. Agora observemos que $u=\beta_{2} v_{1}+\beta_{3} v_{2}$, onde

$$
v_{1}(x, y):= \begin{cases}\frac{\operatorname{sen} \sqrt{\lambda} x}{3}, & \text { quase sempre em } \Omega_{1}, \\ \operatorname{sen} \sqrt{\lambda} x, & \text { quase sempre cm } \Omega_{2}, \\ 0, & \text { quase sempre em } \Omega_{3},\end{cases}
$$

$$
v_{2}(u, y):- \begin{cases}\frac{\operatorname{sen} \sqrt{\lambda} x}{3}, & \text { quase sempre cm } \Omega_{1}, \\ 0, & \text { quase sempre em } \Omega_{2}, \\ \operatorname{sen} \sqrt{\lambda} x, & \text { quase sempre em } \Omega_{3} .\end{cases}
$$

Como $v_{1}$ e $v_{2}$ são linearmente independentes, segue que $\lambda$ possui multiplicidade 2 .

Agora passemos ao caso (2). Suponhamos que $\lambda$ seja um autovalor de $A_{0}$ e seja $u$ um autovetor associado a $\lambda$. Então $u$ c detcrminado polas constantes $\alpha$ e $\beta_{l}, l=1,2,3$ de forma que as condições da Proposição 6.3.1 estejam satisfeitas. Com estamos supondo $\cos a \sqrt{\lambda}=0$, a propricdado (4) da Proposição 6.3 .1 implica que $\alpha=0$. Como $\cos a_{3} \sqrt{\lambda} \neq$ 0 , concluímos que $\beta_{3}=0$. As propriedades (4) e (3) implicam que $\beta_{1}=\beta_{2} / 3$. Utilizando as propriedades (1) e (2) dessa mesma proposição obtemos

$$
u(x, y):= \begin{cases}(1 / 3) \beta_{2} \operatorname{sen} \sqrt{\lambda} x, & \text { quase sempre em } \Omega_{1}, \\ \beta_{2} \operatorname{sen} \sqrt{\lambda} x . & \text { quase sempre em } \Omega_{2}, \\ 0, & \text { quase sempre em } \Omega_{3} .\end{cases}
$$

Reciprocamente, se $u$ é definido como em (6.15), temos que $u$ é não-nulo e, tomando $\alpha=0, \beta_{1}=\beta_{2} / 3$ e $\beta_{3}=0$, temos que as propriedades (2)-(4) da Proposição 6.3 .1 estão satisfeitas e portanto $(\lambda, u)$ é um par autovalor-autovetor de $A_{0}$. É claro que $\lambda$ possui multiplicidade simples. O caso (3) é análogo ao caso (2).

Suponhamos que $j \in \mathbb{N}$ seja tal que $\lambda=\lambda_{0, j}<\lambda_{0, j+1}$. Observemos que nos casos (1), (2) e (3) dessa proposição temos que sen $a \sqrt{\lambda} \neq 0$. Logo, a Proposição 6.3 .3 implica que a fámília $\left(\lambda_{\varepsilon, r}\right)_{\varepsilon>0}$ é monótona docrescente e, em particular, $\lambda_{\varepsilon, r}<\lambda$ para todo $\varepsilon>0$. 


\section{Bibliografia}

[1] A. Ambrosetti e G. Prodi, A Primer on Nonlinear Analysis, Cambridge University Press, New York, 1995.

[2] J. Barros Neto, An Introduction to the Theory of Distributions, Pure and Applied Mathematics, M. Dekker, New York, 1981.

[3] H. Brezis, Análisis Funcional, Alianza Universidad Textos 88, Madrid, 1984.

[4] M. C. Carbinatto e K. P. Rybakowski, Conley Index continuation and thin domains problems, Topol. Methods Nonlinear Anal., 16, (2000), 201-251.

[5] A. N. Carvalho, Equações Parabólicas Semilineares, Notas de Aula, ICMC-USP, São Carlos, 2001.

[6] 1R. Czaja, Differential Equations with Sectorial Operators, Wydawnictwo Uniwersytetu Slaskiego, Katowice, 2002

[7] R. Dautray e J. L. Lions, Mathematical Analysis and Numerical Methods for Science and Technology, Vol. II, Springer-Verlag, Berlin, 1990.

[8] _., Mathematical Analysis and Numerical Methods for Science and Technology, Vol. III, Springer-Verlag, Berlin, 1990.

[9] _. Mathematical Analysis and Numerical Methods for Science and Technology, Vol. V, SpringerVerlag, Berlin, 1990.

[10] D. G. Figueiredo, Lectures on the Ekeland Variational Principle with Applications and Detours, Springer-Verlag, Berlin, 1989.

[11] G. B. Folland, Real Analysis. Modern Techniques and Their Applications, John Willey \& Sons, New York, 1984.

[12] A. Friedman, Partial Diffcrential Equations, Holt, Rinehart and Winston, New York, 1969.

[13] J. K. Hale, Asymptotic behavior of dissipative systems, Math. Surveys Monographs, Vol. 25, Amer. Math. Soc., Providence, 1988.

[14] J. K. Ilale e G. Raugel, Reaction-diffusion equations on thin domains, J. Math. Pures Appl. (9) 71 (1992), 33-95

[15] D. Henry, Geometric Theory of Semilinear Parabolic Equations, Springer-Verlag, Berlin, 1981.

[16] O. Ladyzhenskaya, Attractors for Semigroups and Evolution Equations, Cambridge Univ. Press, Cambridge, UK, 1991

[17] A. Pazy, Semigroups of Linear Operators and Applications to Partial Differential Equations, Springer-Verlag, New York, 1983.

[18] M. Prizzi e K. P. Rybakowski, Inertial manifolds on Squeezed domains, J. Dynam. Differential Equations, 15, No. 1 (2003), 1-48.

[19] __ Some recent results on thin domains problems, Topol. Methods Nonlinear Anal., 14, (1999), $239-255$.

[20] - The effect of domain squeczing upon the dynamics of reaction-diffusion equations, J. Diff. Equations, 173, no2, (2001), 271-320. 